\title{
Asymmetric $\alpha$-Functionalization of 2-Alkyl Azaarenes: Synthesis of Tertiary Fluorides having Vicinal Stereogenic Centers
}

\author{
Arko Das, Harshit Joshi, and Prof. Vinod K. Singh* \\ Department of Chemistry, Indian Institute of Technology Kanpur, Kanpur-208016, Uttar Pradesh (India) \\ E-mail: vinodks@iitk.ac.in
}

\section{Contents:}

General experimental procedure $\quad$ S-2

Materials $\quad S-2$

Synthetic procedure A for $\alpha$-Functionalization of Alkyl Azaarenes $(3,5) \quad S-2$

Synthetic procedure B for $\alpha$-Functionalization of Alkyl Azaarenes (4) S-3

Optimization of reaction conditions $\quad S-3$

Synthetic procedure $C$ and characterization of $2 \mathrm{~s}$ and $2 \mathrm{t} \quad S-4$

Characterization of products 3, 4 and $5 \quad S-5$

$\begin{array}{ll}\text { General procedure and characterization of } 7 & \text { S-19 }\end{array}$

$\begin{array}{lr}\text { General procedure and characterization of } 8 & \text { S-20 }\end{array}$

Crystal structure and data for 3ad and 4ep $\quad$ S-22

Proposed transition state and stereochemical outcome $\quad$ S-25

$\begin{array}{ll}\text { Spectra of products } \mathbf{3}, \mathbf{4} \text { and } \mathbf{5} & \text { S-26 }\end{array}$

HPLC spectra of products $3, \mathbf{4}$ and $\mathbf{5} \quad$ S-89

$\begin{array}{lr}\text { References } & \text { S-120 }\end{array}$ 


\section{General experimental procedure:}

All reactions were carried out under an atmosphere of nitrogen in oven-dried glassware with magnetic stirring. ${ }^{1} \mathrm{H}$ and ${ }^{13} \mathrm{C}$ NMR spectra were recorded on jeol $(500 \mathrm{MHz}$ and $400 \mathrm{MHz})$ spectrometers in $\mathrm{CDCl}_{3}$. Chemical shifts are reported in delta $(\delta)$ units, in parts per million (ppm). Tetramethyl silane was used as the internal standard for ${ }^{1} \mathrm{H}$ and ${ }^{13} \mathrm{C}$ NMR respectively. Coupling constants were reported in $\mathrm{Hz}$. Splitting patterns are designated as s for singlet; $d$ for doublet; $t$ for triplet; $q$ for quartet; $d d$ for doublet of doublet; $m$ for multiplet. Mass spectrometric analysis was done by the ESI-TOF method. Routine monitoring of reactions was performed using precoated silica gel TLC plates from E-Merck. All the chromatographic separations were carried out by using silica gel (Merck's, 230-400 mesh). Enantiomeric excess was determined by HPLC analysis on Daicel chiral columns using iso-propanol and n-hexane as eluent at $25{ }^{\circ} \mathrm{C}$. Optical rotations were measured on a commercially available automatic polarimeter. Melting points were recorded on a digital melting point apparatus.

Compound 3ad and 4ep were crystallized by slow evaporation of $\mathrm{CHCl}_{3} / \mathrm{Hexane}(1: 1)$ and the X-ray crystallography data were collected on a Bruker SMART APEX CCD diffractometer equipped with CRYO Industries low-temperature apparatus and intensity data were collected using graphite-monochromated Mo Ka radiation $(\lambda=0.71073 \AA)$ at $100 \mathrm{~K}$. The CCDC number of complex 3ad and 4ep are 2110157 and 2110161 contains the supplementary crystallographic data for this paper. These data can be obtained free of charge via www.ccdc.cam.ac.uk/conts/retrieving.html (or from the Cambridge Crystallographic Data Centre, 12 Union Road, Cambridge CB21EZ, UK; fax: (+44)1223- 336-033; or deposit@ccdc.cam.ac.uk).

\section{Materials:}

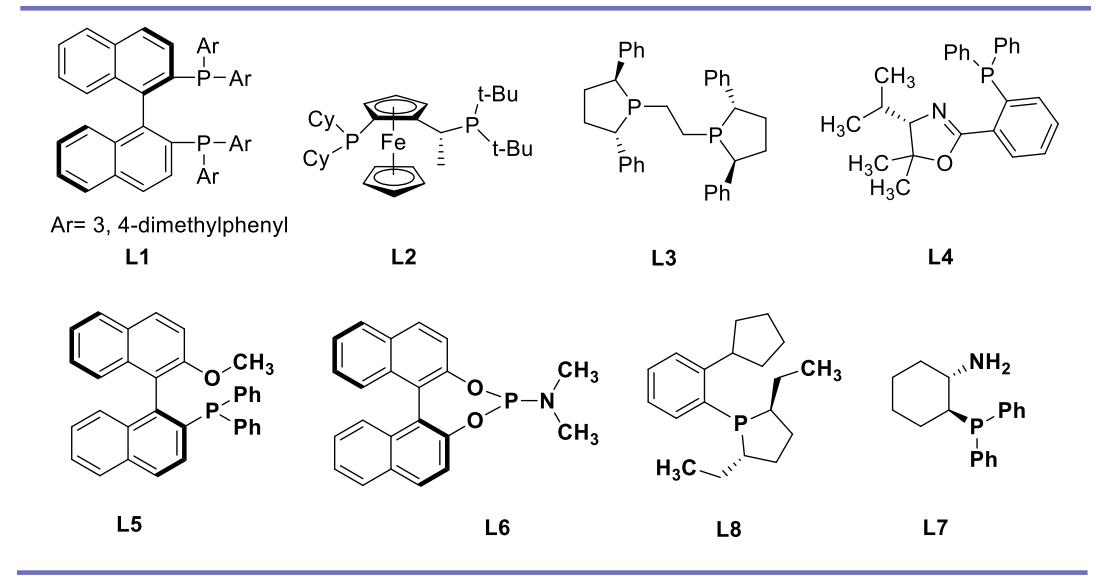

Ligands L1-8 were commercially available. $\alpha, \beta$-unsaturated 2-acyl imidazoles 2 were prepared according to literature known procedure. ${ }^{1} \mathbf{2} \mathbf{s}$ and $\mathbf{2 t}$ were prepared according to the procedure C. Fluorinated azaarenes 1a-e were prepared according to the literature known procedure. ${ }^{2} \mathrm{DBU}$, other bases and all the metal triflates were commercially available.

\section{Synthetic procedure A for $\alpha$-Functionalization of Alkyl Azaarenes $(3,5)$ :}

To an oven-dry round bottom flask, equipped with a magnetic stir bar, $\mathrm{Cu}(\mathrm{MeCN})_{4} \mathrm{PF}_{6}(5 \mathrm{~mol} \%)$, ligand $\mathbf{L} 3(5.5 \mathrm{~mol} \%)$ were added, dissolve in dry THF (1 mL), and stirred for 30 mins under nitrogen atmosphere at room temperature. Pyridinyl fluoroacetates (1a, 1b, 1c, 1d) $(0.2 \mathrm{mmol})$, and DBU $(0.01 \mathrm{mmol})$ were added sequentially into the reaction mixture and continue stirring at the same temperature $\left(25^{\circ} \mathrm{C}\right)$. After 10 mins $\alpha, \beta$-unsaturated 2-acyl imidazoles $2(0.24$ mmol) were also added to the reaction mixture. After complete consumption of the starting material (monitored by TLC, around 15 hours), the reaction mixture was passed through a small silica plague to remove the catalyst. The resulting mixture was then purified by flash column chromatography to get the pure products $\mathbf{3}$ and $\mathbf{5}$. 
Scale-up reaction: To an oven-dry round bottom flask, equipped with a magnetic stir bar, $\mathrm{Cu}(\mathrm{MeCN})_{4} \mathrm{PF}_{6}(37 \mathrm{mg}, 5 \mathrm{~mol}$ $\%$ ), ligand L3 (55 mg, $5.5 \mathrm{~mol} \%$ ) were added, dissolve in dry THF (8 mL), and stirred for 30 mins under nitrogen atmosphere at room temperature. Pyridinyl fluoroacetates $1 \mathrm{a}$ ( $366 \mathrm{mg}, 2 \mathrm{mmol}$ ), and DBU (15 mg, $0.1 \mathrm{mmol}$ ) were added sequentially into the reaction mixture and continue stirring at the same temperature $\left(25^{\circ} \mathrm{C}\right)$. After 10 mins substituted $\alpha, \beta$ unsaturated 2-acyl imidazoles $2 \mathbf{d}(699 \mathrm{mg}, 2.4 \mathrm{mmol})$ were also added to the reaction mixture. After complete consumption of the starting material (monitored by TLC, around 15 hours), the reaction mixture was passed through a small silica plague to remove the catalyst. The resulting mixture was then purified by flash column chromatography to get the pure products 3 ad $(901 \mathrm{mg}, 95 \%$ yield, $98 \%$ ee, $>20: 1 \mathrm{dr}$ ).

\section{Synthetic procedure for racemic reactions:}

Triphenylphosphine (20 mol \%) or rac-BINAP $(20 \mathrm{~mol} \%)$ were used as the ligand with $\mathrm{Cu}(\mathrm{MeCN})_{4} \mathrm{PF}_{6}(20 \mathrm{~mol} \%)$ as the Lewis acid for the synthesis of racemic compounds. Although, these compounds were obtained with lower yields. Diastereomers of some of these racemic products were separated in the column, but some of these diastereomers were inseparable in the column.

\section{Synthetic procedure B for $\alpha$-Functionalization of Alkyl Azaarenes (4):}

To an oven-dry round bottom flask, equipped with a magnetic stir bar, $\mathrm{Cu}(\mathrm{MeCN})_{4} \mathrm{PF}_{6}(5 \mathrm{~mol} \%)$, ligand $\mathbf{L} 3(5.5 \mathrm{~mol} \%)$ were added, dissolve in dry THF (1 mL), and stirred for 30 mins under nitrogen atmosphere at room temperature. The round bottom flask containing the catalyst mixture was then cool down to $-20{ }^{\circ} \mathrm{C}$. Benzothiazolyl fluoroacetate $1 \mathrm{e}(0.2$ $\mathrm{mmol})$, and $\mathrm{DBU}(0.01 \mathrm{mmol})$ were added sequentially into the reaction mixture and continue stirring at the same temperature $\left(-20^{\circ} \mathrm{C}\right)$. After 10 mins $\alpha, \beta$-unsaturated 2-acyl imidazoles $2(0.24 \mathrm{mmol})$ were also added to the reaction mixture. After complete consumption of the starting material (monitored by TLC, around 20 hours), the reaction mixture was passed through a small silica plague to remove the catalyst. The resulting mixture was then purified by flash column chromatography to get the pure products 4 .

\section{Table 1. Optimization of reaction conditions ${ }^{a}$}

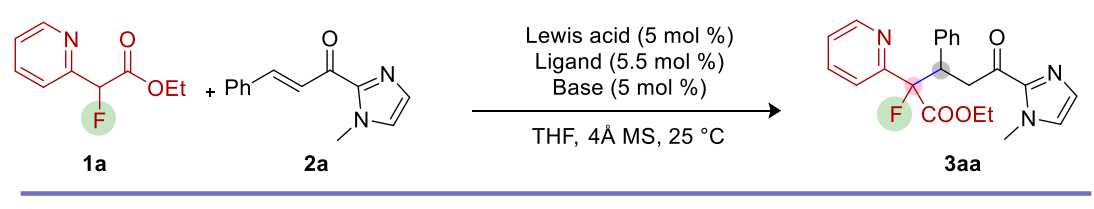

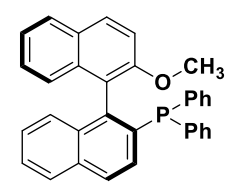

L5

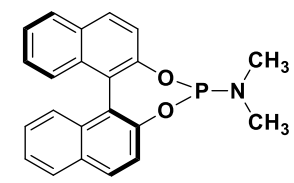

L6<smiles>CC[C@H]1CC[C@@H](CC)P1c1ccccc1C1CCCC1</smiles>

L8

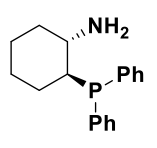

L7

\begin{tabular}{|l|l|l|l|l|l|l|}
\hline entry & catalyst & ligand & base & yield(\%) & ee $(\%)^{c}$ & dr \\
\hline 1 & $\mathrm{Cu}\left(\mathrm{CH}_{3} \mathrm{CN}\right)_{4} \mathrm{PF}_{6}$ & L5 & DBU & 96 & 53 & $2: 1$ \\
\hline 2 & $\mathrm{Cu}\left(\mathrm{CH}_{3} \mathrm{CN}\right)_{4} \mathrm{PF}_{6}$ & $\mathbf{L 8}$ & DBU & 82 & 27 & $1: 10$ \\
\hline 3 & $\mathrm{Cu}\left(\mathrm{CH}_{3} \mathrm{CN}\right)_{4} \mathrm{PF}_{6}$ & L6 & DBU & 25 & nd & $1: 1$ \\
\hline 4 & $\mathrm{Cu}\left(\mathrm{CH}_{3} \mathrm{CN}\right)_{4} \mathrm{PF}_{6}$ & $\mathbf{L 7}$ & DBU & 24 & 13 & $1: 10$ \\
\hline 5 & $\mathrm{Cu}\left(\mathrm{CH}_{3} \mathrm{CN}\right)_{4} \mathrm{PF}_{6}$ & $\mathbf{L 8}$ & N-Methylmorpholine & 44 & 13 & $1: 3$ \\
\hline
\end{tabular}




\begin{tabular}{|l|l|l|l|l|l|l|}
\hline 6 & $\mathrm{Cu}\left(\mathrm{CH}_{3} \mathrm{CN}\right)_{4} \mathrm{PF}_{6}$ & $\mathbf{L 8}$ & PEMPIDINE (PMP) & 85 & 11 & $1: 2$ \\
\hline 7 & $\mathrm{Cu}\left(\mathrm{CH}_{3} \mathrm{CN}\right)_{4} \mathrm{PF}_{6}$ & $\mathbf{L 8}$ & DIPEA & 89 & 7 & $1: 4$ \\
\hline 8 & $\mathrm{Cu}\left(\mathrm{CH}_{3} \mathrm{CN}\right)_{4} \mathrm{PF}_{6}$ & $\mathbf{L 8}$ & $\mathrm{K}_{2} \mathrm{CO}_{3}$ & 53 & 13 & $1: 3$ \\
\hline 9 & $\mathrm{Cu}\left(\mathrm{CH}_{3} \mathrm{CN}\right)_{4} \mathrm{PF}_{6}$ & $\mathbf{L 8}$ & $\mathrm{Cs}_{2} \mathrm{CO}_{3}$ & 80 & 15 & $1: 2$ \\
\hline 10 & $\mathrm{Ni}(\text { acac })_{2}$ & $\mathbf{L 8}$ & DBU & 24 & 9 & $1: 20$ \\
\hline 11 & $\mathrm{Ag}(\mathrm{OTf})$ & $\mathbf{L 8}$ & DBU & 71 & 19 & $1: 2$ \\
\hline 12 & $\mathrm{Co}(\text { acac })_{2}$ & $\mathbf{L 8}$ & DBU & 91 & 4 & $1: 1$ \\
\hline
\end{tabular}

aReaction conditions: 1a $(0.1 \mathrm{mmol}, 1$ equiv), 2 a ( $0.12 \mathrm{mmol}, 1.2$ equiv), metal (5 mol \%), Ligand (10 mol \%), base (5 mol \%), $15 \mathrm{~h}$. ${ }^{b}$ Yield and dr were determined from ${ }^{1} \mathrm{H}$ NMR of the crude reaction mixture with acenaphthene as an internal standard. ${ }^{c}$ ee was determined by HPLC analysis with chiral column.

\section{Synthetic procedure $C$ and characterization of $2 s$ and $2 t$ :}

2-acetyl-1-methylimidazole (I) (1.24 g, $10.0 \mathrm{mmol}, 1.0$ equiv) and $\mathrm{EtOH}$ (20 mL) were added to a $100 \mathrm{~mL}$ RBF followed by the desired aromatic aldehyde ( $10.0 \mathrm{mmol}, 1.0$ equiv) and a catalytic amount of $\mathrm{KOH}$ (2 pellets, $100 \mathrm{mg}$ ). The solution was stirred for $12 \mathrm{~h}$ then transferred to a separatory funnel. Saturated $\mathrm{NaCl}(30 \mathrm{~mL})$ and $\mathrm{H}_{2} \mathrm{O}(10 \mathrm{~mL})$ were added, and the mixture was extracted with EtOAc $(4 \times 75 \mathrm{~mL})$. The combined organic extracts were dried over sodium sulphate, filtered, and concentrated on a rotatory evaporator. The resulting residue was purified by flash column chromatography on silica gel.<smiles>Cn1ccnc1C(=O)C=CC=Cc1ccc(Br)cc1</smiles>

(2E,4E)-5-(4-bromophenyl)-1-(1-methyl-1H-imidazol-2-yl)penta-2,4-dien-1-one (2s): The compound was prepared from (E)-3-(4-bromophenyl)acrylaldehyde $(2.1 \mathrm{~g}, 10 \mathrm{mmol})$ as the starting material. The desired product was isolated by silica gel column chromatography (15:85 = EtOAc/petroleum ether, v/v) to afford a yellow solid (2.4 g, 75\% yield); $\mathbf{m p}$ 130-133 ${ }^{\circ} \mathrm{C} ;{ }^{1} \mathrm{H}$ NMR $\left(500 \mathrm{MHz}, \mathrm{CDCl}_{3}\right) \delta 7.65-7.52(\mathrm{~m}, 2 \mathrm{H}), 7.51-7.45(\mathrm{~m}, 2 \mathrm{H}), 7.36-7.31(\mathrm{~m}, 2 \mathrm{H}), 7.19(\mathrm{~s}, 1 \mathrm{H})$, $7.07-6.96(\mathrm{~m}, 2 \mathrm{H}), 6.92(\mathrm{~d}, J=15.5 \mathrm{~Hz}, 1 \mathrm{H}), 4.07$ (s, 3H). ${ }^{13} \mathrm{C} \mathrm{NMR}\left(126 \mathrm{MHz}, \mathrm{CDCl}_{3}\right) \delta 180.6$ (s), 144.1 (s), 142.9 (s), 140.1 (s), 135.2 (s), 132.1 (s), 129.4 (s), 128.7 (s), 127.9 (s), 127.2 (d, J = 6.5 Hz), 123.2 (s), 36.4 (s). HRMS (ESI, m/z): $[\mathrm{M}+\mathrm{H}]^{+}$calculated for $\mathrm{C}_{15} \mathrm{H}_{14} \mathrm{BrN}_{2} \mathrm{O}: 317.0284$; found: 317.0282 . 


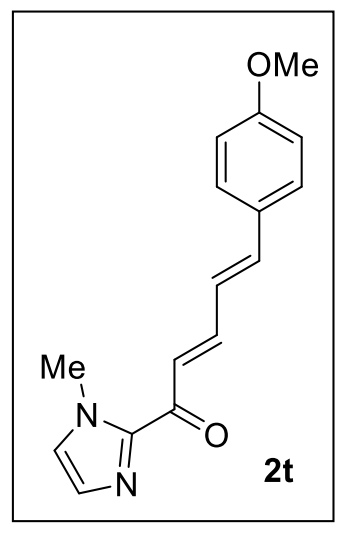

(2E,4E)-5-(4-methoxyphenyl)-1-(1-methyl-1H-imidazol-2-yl)penta-2,4-dien-1-one (2t): The compound was prepared from (E)-3-(4-methoxyphenyl)acrylaldehyde $(1.6 \mathrm{~g}, 10 \mathrm{mmol})$ as the starting material. The desired product was isolated by silica gel column chromatography (15:85 = EtOAc/petroleum ether, v/v) to afford a dark red solid $(2.2 \mathrm{~g}, 82 \%$ yield); mp 140-143 ${ }^{\circ} \mathrm{C} ;{ }^{1} \mathbf{H}$ NMR $\left(500 \mathrm{MHz}, \mathrm{CDCl}_{3}\right) \delta 7.60-7.50(\mathrm{~m}, 2 \mathrm{H}), 7.39(\mathrm{~d}, J=8.7 \mathrm{~Hz}, 2 \mathrm{H}), 7.15(\mathrm{~s}, 1 \mathrm{H}), 7.01(\mathrm{~s}, 1 \mathrm{H})$, $6.92-6.84(\mathrm{~m}, 4 \mathrm{H}), 4.02$ (s, 3H), 3.78 (s, 3H). ${ }^{13} \mathrm{C}$ NMR (126 MHz, $\left.\mathrm{CDCl}_{3}\right) \delta 180.7$ (s), 160.6 (s), 144.1 (s), 141.7 (s), 129.1 (s), 129.1 (s), 128.9 (s), 127.0 (s), 125.4 (s), 125.2 (s), 114.4 (s), 55.4 (s), 36.4 (s). HRMS (ESI, m/z): [M + H] calculated for $\mathrm{C} 16 \mathrm{H} 17 \mathrm{~N} 2 \mathrm{O} 2: 269.1285$; found: 269.1289 .

\section{Synthetic procedure D and characterization of 1:}

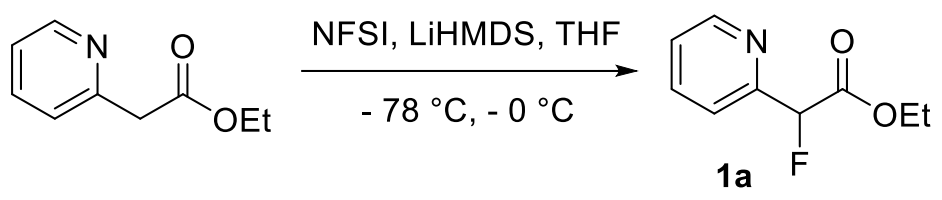

The preparation of $1 \mathrm{a}$ as an example: To a solution of LHMDS (10.0 mL, $1 \mathrm{M}$ in THF, $10.0 \mathrm{mmol}, 1.00$ equiv) in THF (20 $\mathrm{mL}$ ) at $-78^{\circ} \mathrm{C}$ was added methyl 2-pyridylacetate $(1.35 \mathrm{~mL}, 1.51 \mathrm{~g}, 10.0 \mathrm{mmol}, 1.00$ equiv) in THF (10 mL) dropwise. After being stirred at $0^{\circ} \mathrm{C}$ for $40 \mathrm{~min}$, the mixture was cooled to $-78^{\circ} \mathrm{C}$. NFSI (3.15 g, $10.0 \mathrm{mmol}, 1.00$ equiv) in THF (30 mL) was added at $-78^{\circ} \mathrm{C}$. Thereafter, the mixture was stirred at $0{ }^{\circ} \mathrm{C}$ for $1 \mathrm{~h}$. Saturated aqueous $\mathrm{NH}_{4} \mathrm{Cl}$ solution $(10 \mathrm{~mL})$, water $(40 \mathrm{~mL})$, and EtOAc $(30 \mathrm{~mL})$ were added sequentially to the mixture. The organic layer was separated, and the aqueous layer was extracted with EtOAc $(40 \mathrm{~mL} \times 2)$. The organic layers were combined and evaporated under vacuum to be almost dry (around $5 \mathrm{~mL}$ left). The mixture was diluted with $\mathrm{Et}_{2} \mathrm{O}(40 \mathrm{~mL})$ to afford a cloudy yellow solution, which was then filtered through a short column of silica gels ( $3 \mathrm{~cm}$ height). The column was flashed with $\mathrm{Et}_{2} \mathrm{O}(20 \mathrm{~mL} \times 2)$. After removal of solvent under vacuum, the crude mixture was purified by flash column chromatography ( 0 to $50 \%$ EtOAc in hexanes) to give $1 \mathrm{a}$ as a yellow oil $(1.05 \mathrm{~g}, 6.21 \mathrm{mmol}, 62 \%){ }^{2}$

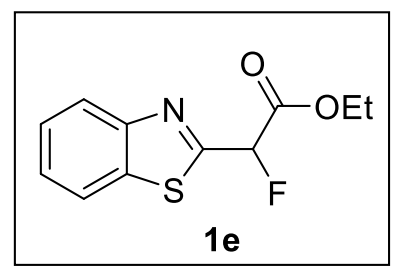

Ethyl 2-(benzo[d]thiazol-2-yl)-2-fluoroacetate (1e): Yellow solid, 52\% yield. ${ }^{1} \mathbf{H}$ NMR $\left(500 \mathrm{MHz}, \mathrm{CDCl}_{3}\right) \delta 8.08$ (d, $J=$ $8.2 \mathrm{~Hz}, 1 \mathrm{H}), 7.90(\mathrm{~d}, J=8.1 \mathrm{~Hz}, 1 \mathrm{H}), 7.54-7.38(\mathrm{~m}, 2 \mathrm{H}), 6.19(\mathrm{~d}, J=47.4 \mathrm{~Hz}, 1 \mathrm{H}), 4.37-4.27(\mathrm{~m}, 2 \mathrm{H}), 1.29(\mathrm{t}, J=7.1$ $\mathrm{Hz}, 3 \mathrm{H}) .{ }^{13} \mathrm{C}$ NMR $\left(125 \mathrm{MHz}, \mathrm{CDCl}_{3}\right) \delta 165.7$ (d, $\left.J=25.9 \mathrm{~Hz}\right), 162.5$ (d, J=26.8 Hz), 152.7 (s), 135.4 (s), 126.7 (s), 126.3 
(s), 124.1 (s), 122.0 (s), 87.1 (d, $J=187.7 \mathrm{~Hz}$ ), 62.9 (s), 14.1 (s). HRMS (ESI, m/z): [M + H] calculated for $\mathrm{C}_{11} \mathrm{H}_{11} \mathrm{FNO}_{2} \mathrm{~S}$ : 240.0489; found: 240.0498

\section{Characterization of products 3, 4 and 5:}

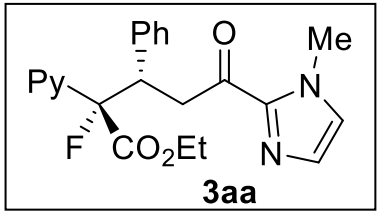

Ethyl (2S,3S)-2-fluoro-5-(1-methyl-1H-imidazol-2-yl)-5-oxo-3-phenyl-2-(pyridin-2-yl)pentanoate (3aa): The compound was prepared following the synthetic procedure $A$ from pyridinyl fluoroacetate $1 \mathrm{a}$ as limiting starting material (36 mg, $0.20 \mathrm{mmol})$. The desired product was isolated by silica gel column chromatography $(30: 70=\mathrm{EtOAc} /$ petroleum ether, v/v) to afford a light yellow gel (76 mg, 97\% yield); ${ }^{1} \mathrm{H}$ NMR $\left(500 \mathrm{MHz}, \mathrm{CDCl}_{3}\right): \delta 8.53(\mathrm{~d}, \mathrm{~J}=3.6 \mathrm{~Hz}, 1 \mathrm{H}), 7.39(\mathrm{t}$, $J=7.7 \mathrm{~Hz}, 1 \mathrm{H}), 7.21-6.83(\mathrm{~m}, 9 \mathrm{H}), 4.76(\mathrm{dd}, J=34.6,6.6 \mathrm{~Hz}, 1 \mathrm{H}), 4.35-4.11(\mathrm{~m}, 3 \mathrm{H}), 3.82(\mathrm{~s}, 3 \mathrm{H}), 3.52(\mathrm{~d}, J=14.5$ $\mathrm{Hz}, 1 \mathrm{H}), 1.23$ (t, $J=7.0 \mathrm{~Hz}, 3 \mathrm{H}) .{ }^{19} \mathrm{~F}$ NMR $\left(376 \mathrm{MHz}, \mathrm{CDCl}_{3}\right) \delta-175.64$ (d, $\left.J=34.8 \mathrm{~Hz}\right) .{ }^{13} \mathrm{C} \mathrm{NMR}\left(125 \mathrm{MHz}, \mathrm{CDCl}_{3}\right): \delta$ 189.3 (s), 168.6 (d, $J=24.6 \mathrm{~Hz}$ ), 156.4 (d, $J=27.8 \mathrm{~Hz}$ ), 148.7 (s), 142.9 (s), 138.0 (s), 136.3 (s), 129.8 (s), 128.8 (s), 127.7 (s), 126.7 (s), 122.9 (s), 120.1 (d, $J=9.1 \mathrm{~Hz}$ ), 100.1 (d, $J=196.2 \mathrm{~Hz}$ ), 62.4 (s), 46.2 (d, J = 18.9 Hz), 40.2 (s), 36.1

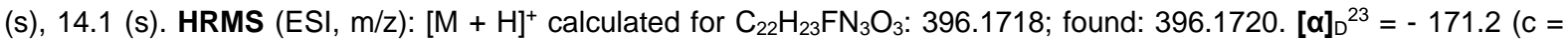
$0.07, \mathrm{CHCl}_{3}, 99 \%$ ee, $>20: 1 \mathrm{dr}$ ); HPLC (IA3, IPA/n-hexane $=30 / 70$, flow rate $\left.=0.8 \mathrm{~mL} / \mathrm{min}, \mathrm{I}=254 \mathrm{~nm}\right) \mathrm{t}_{\mathrm{R}}=13.05 \mathrm{~min}$ (minor), $22.22 \mathrm{~min}$ (major)

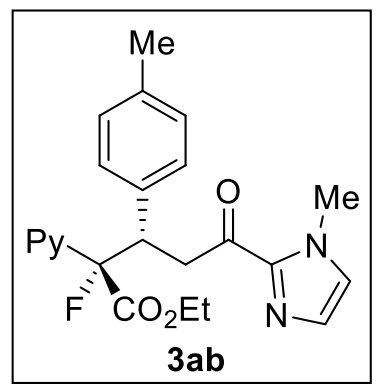

Ethyl (2S,3S)-2-fluoro-5-(1-methyl-1H-imidazol-2-yl)-5-oxo-2-(pyridin-2-yl)-3-(p-tolyl)pentanoate (3ab): The compound was prepared following the synthetic procedure $A$ from pyridinyl fluoroacetate $1 \mathbf{a}$ as limiting starting material $(36 \mathrm{mg}, 0.20 \mathrm{mmol})$. The desired product was isolated by silica gel column chromatography $(30: 70=\mathrm{EtOAc} /$ petroleum ether, v/v) to afford a light yellow gel (79 mg, 96\% yield); mp 108-112 ${ }^{\circ} \mathrm{C} ;{ }^{1} \mathbf{H} \mathbf{~ N M R}\left(500 \mathrm{MHz}, \mathrm{CDCl}_{3}\right): \delta 8.48$ (d, J=3.2 $\mathrm{Hz}, 1 \mathrm{H}$ ), 7.37 (t, $J=7.7 \mathrm{~Hz}, 1 \mathrm{H}$ ), 6.99 (ddd, $J=44.5,30.6,18.1 \mathrm{~Hz}, 6 \mathrm{H}), 6.75$ (d, $J=7.8 \mathrm{~Hz}, 2 \mathrm{H}$ ), 4.67 (dd, $J=34.6,9.5$ $\mathrm{Hz}, 1 \mathrm{H}), 4.28-4.09(\mathrm{~m}, 3 \mathrm{H}), 3.78(\mathrm{~s}, 3 \mathrm{H}), 3.42(\mathrm{~d}, J=17.0 \mathrm{~Hz}, 1 \mathrm{H}), 2.07(\mathrm{~s}, 3 \mathrm{H}), 1.18(\mathrm{t}, J=7.1 \mathrm{~Hz}, 3 \mathrm{H}) .{ }^{19} \mathrm{~F} \mathrm{NMR}(376$ $\mathrm{MHz}, \mathrm{CDCl}_{3}$ ) $\delta-175.74$ (d, $J=33.6 \mathrm{~Hz}$ ). ${ }^{13} \mathrm{C}$ NMR (125 MHz, $\left.\mathrm{CDCl}_{3}\right): \delta 189.2$ (s), 168.7 (d, $\left.J=25.0 \mathrm{~Hz}\right), 156.4$ (d, $J=$ $28.2 \mathrm{~Hz}$ ), 148.6 (s), 136.5 (s), 136.2 (s), 134.7 (s), 129.6 (s), 128.5 (s), 126.9 (s), 122.9 (s), 120.3 (d, J = 7.9 Hz), 100.1 (d, $J=195.7 \mathrm{~Hz}$ ), 62.4 (s), 45.8 (d, J=19.0 Hz), 40.4 (s), 36.2 (s), 21.0 (s), 14.1 (s). HRMS (ESI, m/z): [M + H] calculated for $\mathrm{C}_{23} \mathrm{H}_{25} \mathrm{FN}_{3} \mathrm{O}_{3}$ : 410.1874; found: 410.1879. [ $\left.\alpha\right]_{D}^{23}=-215.7$ (c=0.71, $\mathrm{CHCl}_{3}, 99 \%$ ee, >20:1 dr); HPLC (IA3, IPA/nhexane $=10 / 90$, flow rate $=0.8 \mathrm{~mL} / \mathrm{min}, \mathrm{I}=254 \mathrm{~nm}$ ) $\mathrm{t}_{\mathrm{R}}=62.36 \mathrm{~min}$ (minor), $99.99 \mathrm{~min}$ (major). 


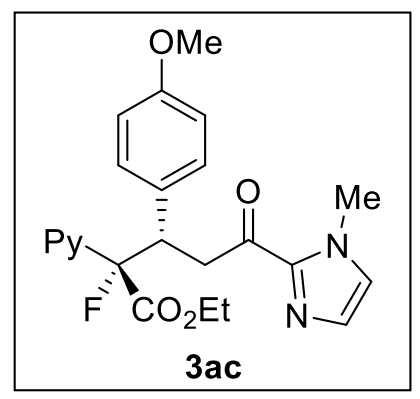

Ethyl (2S,3S)-2-fluoro-3-(4-methoxyphenyl)-5-(1-methyl-1H-imidazol-2-yl)-5-oxo-2-(pyridin-2-yl)pentanoate (3ac): The compound was prepared following the synthetic procedure A from pyridinyl fluoroacetate 1a as limiting starting material $(36 \mathrm{mg}, 0.20 \mathrm{mmol})$. The desired product was isolated by silica gel column chromatography $(40: 60=$ EtOAc/petroleum ether, v/v) to afford a light yellow gel $\left(77 \mathrm{mg}, 91 \%\right.$ yield); ${ }^{1} \mathbf{H} \mathbf{~ N M R}\left(500 \mathrm{MHz}, \mathrm{CDCl}_{3}\right): \delta 8.52(\mathrm{~s}, 1 \mathrm{H})$, $7.46-7.37(\mathrm{~m}, 1 \mathrm{H}), 7.15-7.07(\mathrm{~m}, 2 \mathrm{H}), 7.06-6.99(\mathrm{~m}, 3 \mathrm{H}), 6.93(\mathrm{~s}, 1 \mathrm{H}), 6.52(\mathrm{~d}, J=6.2 \mathrm{~Hz}, 2 \mathrm{H}), 4.70$ (dd, $J=34.7$, $10.7 \mathrm{~Hz}, 1 \mathrm{H}), 4.27-4.15(\mathrm{~m}, 3 \mathrm{H}), 3.81(\mathrm{~s}, 3 \mathrm{H}), 3.61(\mathrm{~s}, 3 \mathrm{H}), 3.44(\mathrm{~d}, J=17.6 \mathrm{~Hz}, 1 \mathrm{H}), 1.21(\mathrm{t}, J=5.7 \mathrm{~Hz}, 3 \mathrm{H}) .{ }^{19} \mathrm{~F} \mathrm{NMR}$ $\left(376 \mathrm{MHz}, \mathrm{CDCl}_{3}\right) \delta-175.79$ (d, J = 34.0 Hz). ${ }^{13} \mathrm{C} \mathrm{NMR}\left(125 \mathrm{MHz}, \mathrm{CDCl}_{3}\right): \delta 189.4$ (s), 168.7 (d, J = 24.8 Hz), $158.2(\mathrm{~s})$, 156.4 (d, $J=27.9 \mathrm{~Hz}$ ), 148.7 (s), 142.9 (s), 136.4 (s), 130.8 (s), 129.9 (s), 128.8 (s), 126.8 (s), 122.9 (s), 120.2 (d, $J=9.4$ Hz), 113.1 (s), 100.2 (d, $J=195.5$ Hz), 62.3 (s), 55.0 (s), 45.5 (d, $J=19.0 \mathrm{~Hz}$ ), 40.3 (s), 36.1 (s), 14.1 (s). HRMS (ESI, $\mathrm{m} / \mathrm{z}):[\mathrm{M}+\mathrm{H}]^{+}$calculated for $\mathrm{C}_{23} \mathrm{H}_{25} \mathrm{FN}_{3} \mathrm{O}_{4}: 426.1824$; found: 426.1825. [a $]_{D}^{23}=-117.35\left(\mathrm{c}=1.51, \mathrm{CHCl}_{3}, 97 \%\right.$ ee, $>20: 1$ $\mathrm{dr}$ ); HPLC (IA3, IPA $/ \mathrm{n}$-hexane = 30/70, flow rate $=0.8 \mathrm{~mL} / \mathrm{min}, \mathrm{I}=254 \mathrm{~nm}$ ) $\mathrm{t}_{\mathrm{R}}=12.27 \mathrm{~min}$ (minor), $30.74 \mathrm{~min}$ (major).<smiles></smiles>

Ethyl (2S,3S)-3-(4-bromophenyl)-2-fluoro-5-(1-methyl-1H-imidazol-2-yl)-5-oxo-2-(pyridin-2-yl)pentanoate (3ad): The compound was prepared following the synthetic procedure A from pyridinyl fluoroacetate 1 a as limiting starting material $(36 \mathrm{mg}, 0.20 \mathrm{mmol})$. The desired product was isolated by silica gel column chromatography $(30: 70=$ EtOAc/petroleum ether, v/v) to afford a light yellow gel (90 mg, 95\% yield); mp 103-106 ${ }^{\circ} \mathrm{C} ;{ }^{1} \mathbf{H ~} \mathbf{~ N M R ~}\left(500 \mathrm{MHz} \mathrm{CDCl}_{3}\right)$ : $\delta 8.48(\mathrm{~d}, J=4.3 \mathrm{~Hz}, 1 \mathrm{H}), 7.40$ (t, $J=7.5 \mathrm{~Hz}, 1 \mathrm{H}), 7.14-7.04(\mathrm{~m}, 4 \mathrm{H}), 7.04-6.95(\mathrm{~m}, 3 \mathrm{H}), 6.91(\mathrm{~s}, 1 \mathrm{H}), 4.71$ (dd, $J=$ 34.4, $10.4 \mathrm{~Hz}, 1 \mathrm{H}), 4.30-4.05(\mathrm{~m}, 3 \mathrm{H}), 3.77(\mathrm{~s}, 3 \mathrm{H}), 3.42$ (d, J = 17.2 Hz, $1 \mathrm{H}), 1.17$ (t, J = 6.7 Hz, 3H). ${ }^{19} \mathrm{~F} \mathrm{NMR}(376$ $\mathrm{MHz}, \mathrm{CDCl}_{3}$ ) $\delta-176.07$ (d, $J=34.6 \mathrm{~Hz}$ ). ${ }^{13} \mathrm{C}$ NMR (125 MHz, $\left.\mathrm{CDCl}_{3}\right): \delta 189.1$ (s), 168.4 (d, $\left.J=24.9 \mathrm{~Hz}\right), 156.0$ (d, $J=$ $28.0 \mathrm{~Hz}$ ), 148.8 (s), 142.8 (s), 137.1 (s), 136.6 (s), 131.6 (s), 130.8 (s), 128.9 (s), 127.1 (s), 123.1 (s), 120.8 (s), 120.1 (d, $J=9.3 \mathrm{~Hz}$ ), $99.8(\mathrm{~d}, J=196.1 \mathrm{~Hz}), 62.5(\mathrm{~s}), 45.6(\mathrm{~d}, J=18.8 \mathrm{~Hz}), 40.1(\mathrm{~s}), 36.1(\mathrm{~s}), 14.1(\mathrm{~s})$. HRMS (ESI, m/z): [M + H] calculated for $\mathrm{C}_{22} \mathrm{H}_{22} \mathrm{BrFN}_{3} \mathrm{O}_{3}$ : 474.0823; found: 474.0829. [ []$_{D}^{23}=-132.4$ (c=0.75, $\mathrm{CHCl}_{3}, 99 \%$ ee, >20:1 dr);

HPLC (IA3, IPA $/ \mathrm{n}$-hexane $=30 / 70$, flow rate $=0.8 \mathrm{~mL} / \mathrm{min}, \mathrm{I}=254 \mathrm{~nm}$ ) $\mathrm{t}_{\mathrm{R}}=13.73 \mathrm{~min}$ (minor), $29.90 \mathrm{~min}$ (major). 


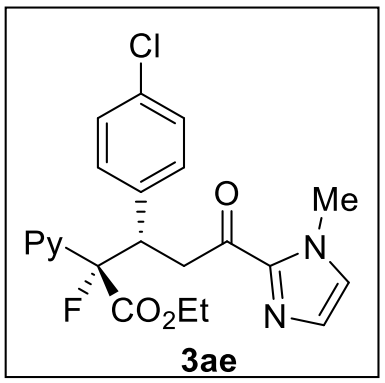

Ethyl (2S,3S)-3-(4-chlorophenyl)-2-fluoro-5-(1-methyl-1H-imidazol-2-yl)-5-oxo-2-(pyridin-2-yl)pentanoate (3ae): The compound was prepared following the synthetic procedure A from pyridinyl fluoroacetate 1 a as limiting starting material $(36 \mathrm{mg}, 0.20 \mathrm{mmol})$. The desired product was isolated by silica gel column chromatography $(30: 70=$ EtOAc/petroleum ether, v/v) to afford a light yellow gel (83 mg, 97\% yield); ${ }^{1} \mathrm{H}$ NMR $\left(400 \mathrm{MHz}, \mathrm{CDCl}_{3}\right): \delta 8.56(\mathrm{~d}, J=4.4$ $\mathrm{Hz}, 1 \mathrm{H}), 7.47$ (td, $J=7.8,1.6 \mathrm{~Hz}, 1 \mathrm{H}), 7.21-7.03(\mathrm{~m}, 5 \mathrm{H}), 7.03-6.84(\mathrm{~m}, 3 \mathrm{H}), 4.80$ (ddd, $J=34.5,11.1,2.9 \mathrm{~Hz}, 1 \mathrm{H})$, $4.36-4.12(\mathrm{~m}, 3 \mathrm{H}), 3.86(\mathrm{~s}, 3 \mathrm{H}), 3.48(\mathrm{dd}, J=17.7,2.9 \mathrm{~Hz}, 1 \mathrm{H}), 1.25(\mathrm{t}, J=7.1 \mathrm{~Hz}, 3 \mathrm{H}) .{ }^{19} \mathrm{~F} \mathrm{NMR}\left(376 \mathrm{MHz}, \mathrm{CDCl}_{3}\right) \delta-$ 176.09 (d, $J=34.1 \mathrm{~Hz}) .{ }^{13} \mathrm{C}$ NMR $\left(100 \mathrm{MHz}, \mathrm{CDCl}_{3}\right): \delta 189.2$ (s), 168.4 (d, $\left.J=24.8 \mathrm{~Hz}\right), 156.1$ (d, $\left.J=27.9 \mathrm{~Hz}\right), 148.9$ (s), 142.9 (s), 136.6 (s), 132.6 (s), 131.2 (s), 129.1 (s), 127.9 (s), 127.0 (s), 123.1 (s), 120.1 (d, J = 9.4 Hz), 99.9 (d, J = 196.2 $\mathrm{Hz}$ ), 62.5 (s), 45.6 (d, J = $19.0 \mathrm{~Hz}$ ), 40.1 (s), 36.1 (s), 14.1 (s). HRMS (ESI, m/z): [M + H] calculated for $\mathrm{C}_{22} \mathrm{H}_{22} \mathrm{CIFN}_{3} \mathrm{O}_{3}$ : 430.1328; found: $430.1335 .[\alpha]_{\mathrm{D}}^{23}=-154.1$ ( $\mathrm{c}=0.44, \mathrm{CHCl}_{3}, 99 \%$ ee, $>20: 1 \mathrm{dr}$ );

HPLC (IA3, IPA $/ \mathrm{n}$-hexane $=30 / 70$, flow rate $=0.8 \mathrm{~mL} / \mathrm{min}, \mathrm{I}=254 \mathrm{~nm}) \mathrm{t}_{\mathrm{R}}=12.42 \mathrm{~min}$ (minor), $28.35 \mathrm{~min}$ (major).

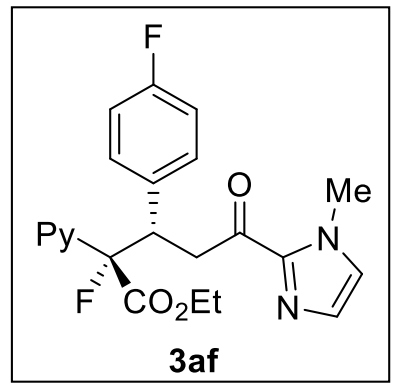

Ethyl (2S,3S)-2-fluoro-3-(4-fluorophenyl)-5-(1-methyl-1H-imidazol-2-yl)-5-oxo-2-(pyridin-2-yl)pentanoate (3af): The compound was prepared following the synthetic procedure A from pyridinyl fluoroacetate 1a as limiting starting material $(36 \mathrm{mg}, 0.20 \mathrm{mmol})$. The desired product was isolated by silica gel column chromatography $(30: 70=$ EtOAc/petroleum ether, v/v) to afford a light yellow gel $\left(75 \mathrm{mg}, 91 \%\right.$ yield); ${ }^{1} \mathbf{H}$ NMR $\left(500 \mathrm{MHz}, \mathrm{CDCl}_{3}\right): \delta 8.48$ (dd, $\mathrm{J}=$ 4.3, 1.2 Hz, 1H), 7.38 (td, $J=7.7,1.7 \mathrm{~Hz}, 1 \mathrm{H}), 7.13-7.02(\mathrm{~m}, 4 \mathrm{H}), 7.02-6.98(\mathrm{~m}, 1 \mathrm{H}), 6.90(\mathrm{~s}, 1 \mathrm{H}), 6.63(\mathrm{t}, J=8.8 \mathrm{~Hz}$, 2H), 4.72 (ddd, $J=34.6,11.1,3.0 \mathrm{~Hz}, 1 \mathrm{H}$ ), $4.24-4.10(\mathrm{~m}, 3 \mathrm{H}), 3.78(\mathrm{~s}, 3 \mathrm{H}), 3.41(\mathrm{dd}, J=17.7,3.0 \mathrm{~Hz}, 1 \mathrm{H}$ ), $1.20-1.15$ (m, 3H). ${ }^{19} \mathrm{~F}$ NMR $\left(376 \mathrm{MHz}, \mathrm{CDCl}_{3}\right) \delta-115.86(\mathrm{~s}),-176.26$ (d, J=34.6 Hz). ${ }^{13} \mathrm{C} \mathrm{NMR}\left(125 \mathrm{MHz}, \mathrm{CDCl}_{3}\right): \delta 189.9$ (s), 168.5 (d, $J=24.7 \mathrm{~Hz}$ ), 161.7 (d, $J=245.0 \mathrm{~Hz}$ ), 156.2 (d, $J=27.9 \mathrm{~Hz}), 148.8$ (s), 142.8 (s), 136.5 (s), 133.7 (s), 131.3 (d, $J=7.5 \mathrm{~Hz}$ ), 128.8 (s), 127.0 (s), 123.0 (s), 120.1 (d, $J=9.3 \mathrm{~Hz}$ ), 114.6 (d, J = 21.2 Hz), 100.0 (d, J = 195.9 Hz), 62.4 (s), 45.5 (d, $J=18.9 \mathrm{~Hz}$ ), 40.3 (s), 36.1 (s), 14.1 (s). HRMS (ESI, m/z): [M + H] $]^{+}$calculated for $\mathrm{C}_{22} \mathrm{H}_{22} \mathrm{~F}_{2} \mathrm{~N}_{3} \mathrm{O}_{3}: 414.1624$; found: $414.1629 .[\alpha]_{D}^{23}=-152.63\left(\mathrm{c}=1.9, \mathrm{CHCl}_{3}, 98 \%\right.$ ee, $\left.>20: 1 \mathrm{dr}\right) ; \operatorname{HPLC}(\mathrm{IA3}$, IPA/n-hexane $=30 / 70$, flow rate $=0.8$ $\mathrm{mL} / \mathrm{min}, \mathrm{I}=254 \mathrm{~nm}$ ) $\mathrm{t}_{\mathrm{R}}=10.61 \mathrm{~min}$ (minor), $27.65 \mathrm{~min}$ (major). 


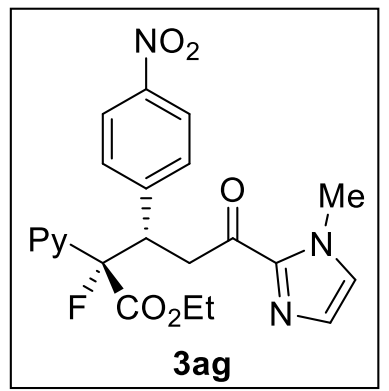

Ethyl (2S,3S)-2-fluoro-5-(1-methyl-1H-imidazol-2-yl)-3-(4-nitrophenyl)-5-oxo-2-(pyridin-2-yl)pentanoate (3ag): The compound was prepared following the synthetic procedure A from pyridinyl fluoroacetate 1a as limiting starting material (36 mg, $0.20 \mathrm{mmol})$. The desired product was isolated by silica gel column chromatography (40:60 = EtOAc/petroleum ether, v/v) to afford a light yellow gel (85 mg, 96\% yield); ${ }^{1} \mathrm{H}$ NMR $\left(400 \mathrm{MHz}, \mathrm{CDCl}_{3}\right): \delta 8.54(\mathrm{~d}, \mathrm{~J}=3.6 \mathrm{~Hz}, 1 \mathrm{H}), 7.86(\mathrm{~d}$, $J=8.9 \mathrm{~Hz}, 2 \mathrm{H}), 7.46(\mathrm{td}, J=7.8,1.7 \mathrm{~Hz}, 1 \mathrm{H}), 7.40-7.33(\mathrm{~m}, 2 \mathrm{H}), 7.17(\mathrm{dd}, J=7.7,1.3 \mathrm{~Hz}, 1 \mathrm{H}), 7.13-7.04(\mathrm{~m}, 2 \mathrm{H})$, $6.97(\mathrm{~s}, 1 \mathrm{H}), 4.96$ (ddd, $J=34.0,11.2,2.9 \mathrm{~Hz}, 1 \mathrm{H}), 4.30-4.18(\mathrm{~m}, 3 \mathrm{H}), 3.84(\mathrm{~s}, 3 \mathrm{H}), 3.54(\mathrm{dd}, J=17.9,3.0 \mathrm{~Hz}, 1 \mathrm{H}), 1.23$ (t, $J=7.2 \mathrm{~Hz}, 3 \mathrm{H}) .{ }^{19} \mathrm{~F}$ NMR $\left(376 \mathrm{MHz}, \mathrm{CDCl}_{3}\right) \delta-175.95(\mathrm{~d}, J=34.1 \mathrm{~Hz}) \cdot{ }^{13} \mathrm{C}$ NMR $\left(100 \mathrm{MHz}, \mathrm{CDCl}_{3}\right): \delta 188.7$ (s), 168.0 (d, $J=24.9 \mathrm{~Hz}$ ), 155.5 (d, J = 28.0 Hz), 149.1 (d, $J=2.6 \mathrm{~Hz}$ ), 146.7 (s), 146.1 (s), 142.6 (s), 136.8 (s), 130.8 (s), 129.2 (s), 127.3 (s), 123.3 (s), 122.9 (s), 120.0 (d, J = 9.6 Hz), 99.5 (d, J = 197.0 Hz), 62.7 (s), 45.9 (d, J = $18.5 \mathrm{~Hz}$ ), 39.9 (d, J

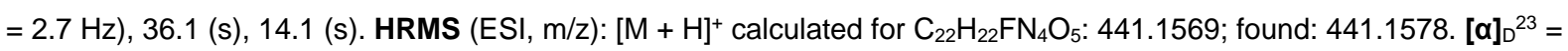
- 145 (c = 2.2, $\mathrm{CHCl}_{3}, 99 \%$ ee, $>20: 1 \mathrm{dr}$ ); HPLC (IA3, IPA $/ \mathrm{n}$-hexane $=30 / 70$, flow rate $\left.=0.8 \mathrm{~mL} / \mathrm{min}, \mathrm{I}=254 \mathrm{~nm}\right) \mathrm{t}_{\mathrm{R}}=$ $17.92 \mathrm{~min}$ (minor), $55.99 \mathrm{~min}$ (major).

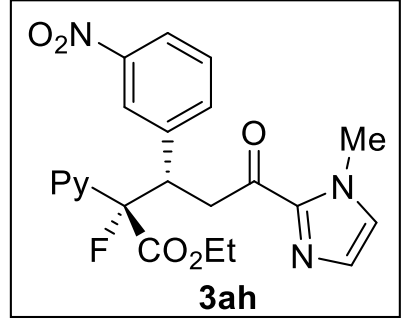

Ethyl (2S,3S)-2-fluoro-5-(1-methyl-1H-imidazol-2-yl)-3-(3-nitrophenyl)-5-oxo-2-(pyridin-2-yl)pentanoate (3ah): The compound was prepared following the synthetic procedure $A$ from pyridinyl fluoroacetate $\mathbf{1 a}$ as limiting starting material (36 mg, $0.20 \mathrm{mmol})$. The desired product was isolated by silica gel column chromatography (40:60 = EtOAc/petroleum ether, v/v) to afford a light yellow gel $\left(84 \mathrm{mg}, 96 \%\right.$ yield); ${ }^{1} \mathrm{H}$ NMR $\left(400 \mathrm{MHz}, \mathrm{CDCl}_{3}\right): \delta 8.60-8.49(\mathrm{~m}, 1 \mathrm{H}), 8.05(\mathrm{~s}, 1 \mathrm{H})$, $7.87-7.78(\mathrm{~m}, 1 \mathrm{H}), 7.54$ (dd, $J=7.9,0.8 \mathrm{~Hz}, 1 \mathrm{H}), 7.44(\mathrm{td}, J=7.8,1.7 \mathrm{~Hz}, 1 \mathrm{H}), 7.23-7.13(\mathrm{~m}, 2 \mathrm{H}), 7.10(\mathrm{~s}, 1 \mathrm{H}), 7.06$ (ddd, $J=7.4,4.9,1.0 \mathrm{~Hz}, 1 \mathrm{H}), 6.97(\mathrm{~s}, 1 \mathrm{H}), 4.96$ (ddd, $J=33.8,11.1,3.0 \mathrm{~Hz}, 1 \mathrm{H}), 4.32-4.14(\mathrm{~m}, 3 \mathrm{H}), 3.84(\mathrm{~s}, 3 \mathrm{H}), 3.57$ $(\mathrm{dd}, J=18.1,3.0 \mathrm{~Hz}, 1 \mathrm{H}), 1.23(\mathrm{t}, J=7.2 \mathrm{~Hz}, 3 \mathrm{H}) .{ }^{19} \mathrm{~F} \mathrm{NMR}\left(376 \mathrm{MHz}, \mathrm{CDCl}_{3}\right) \delta-175.82(\mathrm{~d}, J=33.2 \mathrm{~Hz}) .{ }^{13} \mathrm{C} \mathrm{NMR}(100$ $\mathrm{MHz}_{\mathrm{CDCl}}$ ): $\delta 188.7$ (s), 168.1 (d, $J=25.0 \mathrm{~Hz}$ ), 155.6 (d, J = 27.9 Hz), 150.1 (s), 147.7 (s), 142.6 (s), 140.5 (s), 136.8 (s), 136.1 (s), 129.2 (s), 128.6 (s), 127.2 (s), 124.9 (s), 123.3 (s), 121.9 (s), 120.0 (d, J = 9.5 Hz), 99.6 (d, J = 196.6 Hz), 62.7 (s), 45.7 (d, $J=18.7 \mathrm{~Hz}$ ), 39.9 (d, $J=2.5 \mathrm{~Hz}$ ), 36.1 (s), 14.1 (s). HRMS (ESI, m/z): [M + H] calculated for $\mathrm{C}_{22} \mathrm{H}_{22} \mathrm{FN}_{4} \mathrm{O}_{5}$ : 441.1569; found: 441.1567. [a] $]_{D}^{23}=-130$ (c=2.54, $\mathrm{CHCl}_{3}, 99 \%$ ee, $>20: 1 \mathrm{dr}$ ); HPLC (IA3, IPA/n-hexane $=30 / 70$, flow rate $=0.8 \mathrm{~mL} / \mathrm{min}, \mathrm{I}=254 \mathrm{~nm}$ ) $\mathrm{t}_{\mathrm{R}}=17.47 \mathrm{~min}$ (minor), $31.65 \mathrm{~min}$ (major). 


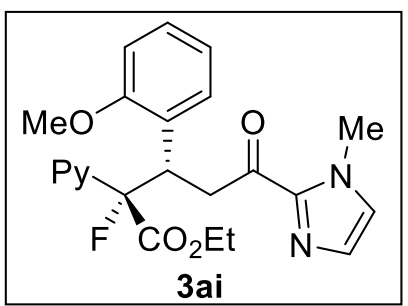

Ethyl (2S,3S)-2-fluoro-3-(2-methoxyphenyl)-5-(1-methyl-1H-imidazol-2-yl)-5-oxo-2-(pyridin-2-yl)pentanoate (3ai): The compound was prepared following the synthetic procedure A from pyridinyl fluoroacetate 1a as limiting starting material $(36 \mathrm{mg}, 0.20 \mathrm{mmol})$. The desired product was isolated by silica gel column chromatography $(40: 60=$ EtOAc/petroleum ether, v/v) to afford a light yellow gel (76 mg, 90\% yield); ${ }^{1} \mathrm{H}$ NMR $\left(500 \mathrm{MHz}, \mathrm{CDCl}_{3}\right): \delta 8.51-8.45$ (m, $1 \mathrm{H}), 7.43-7.38(\mathrm{~m}, 1 \mathrm{H}), 7.37-7.31(\mathrm{~m}, 1 \mathrm{H}), 7.11(\mathrm{~s}, 1 \mathrm{H}), 7.08-6.99(\mathrm{~m}, 2 \mathrm{H}), 6.94(\mathrm{~s}, 1 \mathrm{H}), 6.86(\mathrm{dd}, J=8.0,0.7 \mathrm{~Hz}$, $1 \mathrm{H}), 6.82-6.75(\mathrm{~m}, 1 \mathrm{H}), 6.51-6.42(\mathrm{~m}, 1 \mathrm{H}), 5.18(\mathrm{ddd}, J=34.4,10.8,3.4 \mathrm{~Hz}, 1 \mathrm{H}), 4.31-4.20(\mathrm{~m}, 3 \mathrm{H}), 3.83(\mathrm{~s}, 3 \mathrm{H})$, $3.61(\mathrm{dd}, J=17.8,3.4 \mathrm{~Hz}, 1 \mathrm{H}), 3.36(\mathrm{~s}, 3 \mathrm{H}), 1.26-1.23(\mathrm{~m}, 3 \mathrm{H}) .{ }^{19} \mathrm{~F}$ NMR $\left(376 \mathrm{MHz}, \mathrm{CDCl}_{3}\right) \delta-172.78(\mathrm{~d}, J=34.8 \mathrm{~Hz})$. ${ }^{13} \mathrm{C}$ NMR (125 MHz, $\mathrm{CDCl}_{3}$ ): $\delta 184.8$ (s), 152.7 (s), 151.9 (d, J = 27.4 Hz), 143.3 (s), 138.1 (s), 130.9 (s), 124.7 (s), 123.8 (s), 123.3 (s), 121.9 (s), 121.7 (s), 118.1 (s), 115.8 (d, J = 7.4 Hz), 115.5 (s), 105.5 (s), 95.3 (d, J = 196.3 Hz), 57.5 (s), 50.8 (s), 35.3 (s), 33.0 (d, $J=19.8 \mathrm{~Hz}$ ), 31.4 (s), 9.3 (d, $J=21.9 \mathrm{~Hz})$. HRMS (ESI, m/z): [M + H] calculated for $\mathrm{C}_{23} \mathrm{H}_{25} \mathrm{FN}_{3} \mathrm{O}_{4}$ : 426.1824; found: 426.1825. [ $\left.\alpha\right]_{D}{ }^{23}=-163.1$ ( $\mathrm{c}=0.42, \mathrm{CHCl}_{3}, 97 \%$ ee, $>20: 1 \mathrm{dr}$ ); HPLC (IB3, IPA/n-hexane $=10 / 90$, flow rate $=1 \mathrm{~mL} / \mathrm{min}, \mathrm{I}=254 \mathrm{~nm}$ ) $t_{R}=25.45 \mathrm{~min}$ (minor), $30.32 \mathrm{~min}$ (major).

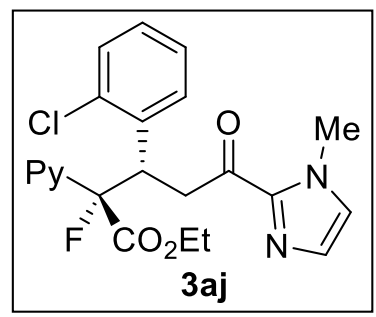

Ethyl (2S,3S)-3-(2-chlorophenyl)-2-fluoro-5-(1-methyl-1H-imidazol-2-yl)-5-oxo-2-(pyridin-2-yl)pentanoate (3aj): The compound was prepared following the synthetic procedure A from pyridinyl fluoroacetate 1a as limiting starting material $(36 \mathrm{mg}, 0.20 \mathrm{mmol})$. The desired product was isolated by silica gel column chromatography $(35: 65=$ EtOAc/petroleum ether, v/v) to afford a light yellow gel (80 mg, 93\% yield); ${ }^{1} \mathrm{H}$ NMR $\left(400 \mathrm{MHz}, \mathrm{CDCl}_{3}\right): \delta 8.42(\mathrm{~d}, J=4.0$ $\mathrm{Hz}, 1 \mathrm{H}), 7.52-7.45(\mathrm{~m}, 1 \mathrm{H}), 7.33(\mathrm{td}, J=7.9,1.7 \mathrm{~Hz}, 1 \mathrm{H}), 7.08-6.95(\mathrm{~m}, 4 \mathrm{H}), 6.94-6.84(\mathrm{~m}, 3 \mathrm{H}), 5.21$ (ddd, $J=33.1$, 10.7, 3.2 Hz, $1 \mathrm{H}$ ), 4.25 (qd, $J=7.1,3.2 \mathrm{~Hz}, 2 \mathrm{H}$ ), 4.11 (dd, $J=17.6,10.7 \mathrm{~Hz}, 1 \mathrm{H}$ ), $3.78(\mathrm{~s}, 3 \mathrm{H}$ ), 3.63 (dd, $J=17.6,3.4 \mathrm{~Hz}$, $1 \mathrm{H}), 1.20(\mathrm{t}, J=7.1 \mathrm{~Hz}, 3 \mathrm{H}) .{ }^{19} \mathrm{~F}$ NMR $\left(376 \mathrm{MHz} \mathrm{CDCl}_{3}\right) \delta-172.45(\mathrm{~d}, J=34.2 \mathrm{~Hz}) .{ }^{13} \mathrm{C} \mathrm{NMR}\left(100 \mathrm{MHz}, \mathrm{CDCl}_{3}\right): \delta 189.1$ (s), 168.4 (d, $J=23.8 \mathrm{~Hz}$ ), 155.9 (d, J=27.6 Hz), 148.6 (s), 142.9 (s), 136.0 (s), 135.8 (s), 130.0 (d, J = 3.1 Hz), 129.2 (s), 129.0 (s), 128.1 (s), 126.9 (s), 126.6 (s), 123.2 (s), 120.2 (d, J = 7.2 Hz), 100.0 (d, J = 196.8 Hz), 62.5 (s), 41.6 (d, J $=19.5 \mathrm{~Hz}), 40.8$ (s), 36.1 (s), 14.1 (s). HRMS (ESI, m/z): $[\mathrm{M}+\mathrm{H}]^{+}$calculated for $\mathrm{C}_{22} \mathrm{H}_{22} \mathrm{CIFN}_{3} \mathrm{O}_{3}$ : 430.1328; found:

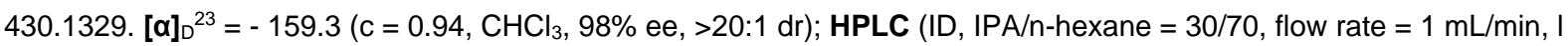
$=254 \mathrm{~nm}$ ) $\mathrm{t}_{\mathrm{R}}=23.18 \mathrm{~min}$ (minor), $54.01 \mathrm{~min}$ (major). 


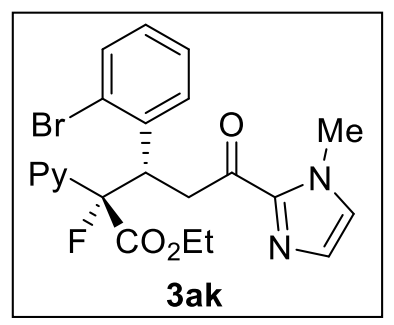

Ethyl (2S,3S)-3-(2-bromophenyl)-2-fluoro-5-(1-methyl-1H-imidazol-2-yl)-5-oxo-2-(pyridin-2-yl)pentanoate (3ak): The compound was prepared following the synthetic procedure A from pyridinyl fluoroacetate 1a as limiting starting material $(36 \mathrm{mg}, 0.20 \mathrm{mmol})$. The desired product was isolated by silica gel column chromatography $(35: 65=$ EtOAc/petroleum ether, v/v) to afford a light yellow gel (88 mg, 93\% yield); ${ }^{1} \mathbf{H} \mathbf{~ N M R}\left(500 \mathrm{MHz}, \mathrm{CDCl}_{3}\right): \delta 8.42(\mathrm{~d}, J=4.4$ $\mathrm{Hz}, 1 \mathrm{H}), 7.50$ (d, J=7.8 Hz, 1H), $7.33(\mathrm{td}, J=7.8,1.6 \mathrm{~Hz}, 1 \mathrm{H}), 7.18(\mathrm{~d}, J=8.0 \mathrm{~Hz}, 1 \mathrm{H}), 7.10(\mathrm{t}, J=7.5 \mathrm{~Hz}, 1 \mathrm{H}), 7.06-$ $6.94(\mathrm{~m}, 2 \mathrm{H}), 6.89(\mathrm{~s}, 1 \mathrm{H}), 6.87-6.76(\mathrm{~m}, 2 \mathrm{H}), 5.18(\mathrm{ddd}, J=32.6,10.6,3.3 \mathrm{~Hz}, 1 \mathrm{H}), 4.34-4.16(\mathrm{~m}, 2 \mathrm{H}), 4.09(\mathrm{dd}, J=$ 17.4, $10.6 \mathrm{~Hz}, 1 \mathrm{H}), 3.78(\mathrm{~s}, 3 \mathrm{H}), 3.64(\mathrm{dd}, J=17.4,3.4 \mathrm{~Hz}, 1 \mathrm{H}), 1.20(\mathrm{t}, J=7.1 \mathrm{~Hz}, 3 \mathrm{H}) .{ }^{19} \mathrm{~F} \mathrm{NMR}\left(376 \mathrm{MHz}, \mathrm{CDCl}_{3}\right) \delta-$ 171.78 (s). ${ }^{13} \mathrm{C}$ NMR (125 MHz, CDCl$): \delta 189.1$ (s), 168.3 (d, $\left.J=23.8 \mathrm{~Hz}\right), 155.9$ (d, J = 27.6 Hz), 148.6 (s), 142.8 (s), 137.8 (s), 136.0 (s), 132.6 (s), 130.2 (s), 128.9 (s), 128.4 (s), 127.2 (s), 126.9 (s), 123.2 (s), 120.4 (d, J = 7.0 Hz), 100.0 (d, $J=196.9 \mathrm{~Hz}$ ), 62.4 (s), 44.5 (d, $J=19.4 \mathrm{~Hz}$ ), 41.1 (s), 36.1 (s), 14.1 (s). HRMS (ESI, m/z): [M + H] $]^{+}$calculated for $\mathrm{C}_{22} \mathrm{H}_{22} \mathrm{BrFN}_{3} \mathrm{O}_{3}$ : 474.0823; found: 474.0830. [ $\left.\alpha\right]_{D}^{23}=-132.8$ (c=0.75, $\mathrm{CHCl}_{3}, 98 \%$ ee, >20:1 dr); HPLC (IB3, IPA/nhexane $=10 / 90$, flow rate $=1 \mathrm{~mL} / \mathrm{min}, \mathrm{I}=254 \mathrm{~nm}$ ) $\mathrm{t}_{\mathrm{R}}=17.31 \mathrm{~min}$ (minor), $19.60 \mathrm{~min}$ (major).

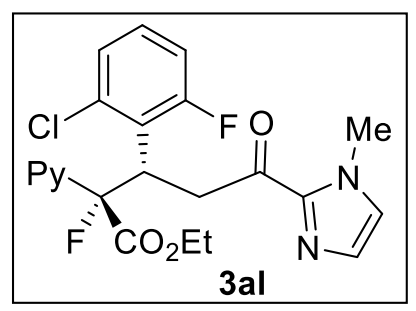

Ethyl (2S,3S)-3-(2-chloro-6-fluorophenyl)-2-fluoro-5-(1-methyl-1H-imidazol-2-yl)-5-oxo-2-(pyridin-2-yl)pentanoate (3al): The compound was prepared following the synthetic procedure A from pyridinyl fluoroacetate 1a as limiting starting material $(36 \mathrm{mg}, 0.20 \mathrm{mmol})$. The desired product was isolated by silica gel column chromatography $(40: 60=$ EtOAc/petroleum ether, v/v) to afford a light yellow gel (80 mg, $90 \%$ yield); ${ }^{1} \mathbf{H}$ NMR $\left(500 \mathrm{MHz}, \mathrm{CDCl}_{3}\right): \delta 8.46(\mathrm{~d}, J=4.3$ $\mathrm{Hz}, 1 \mathrm{H}), 7.43(\mathrm{t}, J=7.6 \mathrm{~Hz}, 1 \mathrm{H}), 7.17-7.04(\mathrm{~m}, 3 \mathrm{H}), 7.01-6.92(\mathrm{~m}, 2 \mathrm{H}), 6.90-6.77(\mathrm{~m}, 2 \mathrm{H}), 5.37$ (ddd, $J=31.6,10.0$, $3.3 \mathrm{~Hz}, 1 \mathrm{H}), 4.39-4.21(\mathrm{~m}, 3 \mathrm{H}), 3.90-3.79(\mathrm{~m}, 4 \mathrm{H}), 1.24(\mathrm{t}, J=7.1 \mathrm{~Hz}, 3 \mathrm{H}) .{ }^{19} \mathrm{~F} \mathrm{NMR}\left(376 \mathrm{MHz}, \mathrm{CDCl}_{3}\right) \delta-105.57(\mathrm{~d}$, $J=56.1 \mathrm{~Hz}$ ), -167.92 (s). ${ }^{13} \mathrm{C}$ NMR (125 MHz, $\left.\mathrm{CDCl}_{3}\right): \delta 189.6$ (s), 168.4 (d, J = 23.9 Hz), 163.5 (s), 161.5 (s), 156.2 (d, $J=27.7 \mathrm{~Hz}$ ), 148.7 (s), 142.6 (s), 137.4 (d, J=7.7 Hz), 136.2 (s), 128.9 (d, J=8.8 Hz), 126.8 (s), 125.2 (s), 124.2 (d, $J=$ $16.6 \mathrm{~Hz}$ ), 123.3 (s), 119.9 (d, $J=8.1 \mathrm{~Hz}$ ), 114.9 (d, J = 24.2 Hz), 99.5 (d, J=200.4 Hz), 62.5 (s), 41.2 (d, J = 20.4 Hz),

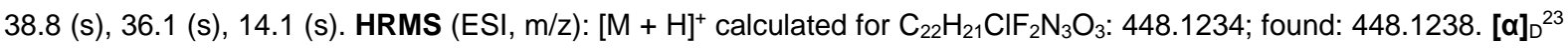
$=-179.6\left(\mathrm{c}=1.17, \mathrm{CHCl}_{3}, 99 \%\right.$ ee, $\left.>20: 1 \mathrm{dr}\right) ; \mathrm{HPLC}(\mathrm{IA}, \mathrm{IPA} / \mathrm{n}$-hexane $=20 / 80$, flow rate $=1 \mathrm{~mL} / \mathrm{min}, \mathrm{I}=254 \mathrm{~nm}) \mathrm{t}_{\mathrm{R}}=$ $17.33 \mathrm{~min}$ (minor), $20.43 \mathrm{~min}$ (major). 


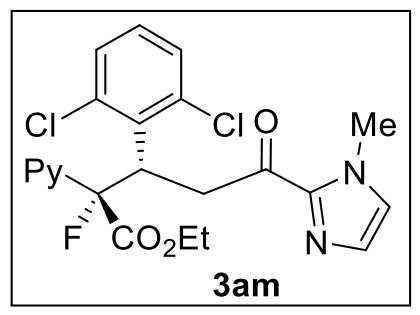

Ethyl (2S,3S)-3-(2,6-dichlorophenyl)-2-fluoro-5-(1-methyl-1H-imidazol-2-yl)-5-oxo-2-(pyridin-2-yl)pentanoate (3am): The compound was prepared following the synthetic procedure A from pyridinyl fluoroacetate 1a as limiting starting material $(36 \mathrm{mg}, 0.20 \mathrm{mmol})$. The desired product was isolated by silica gel column chromatography $(40: 60=$ EtOAc/petroleum ether, v/v) to afford a light yellow gel (77 mg, 83\% yield); $\mathbf{m p} 98-10{ }^{\circ} \mathrm{C} ;{ }^{1} \mathbf{H ~ N M R}\left(500 \mathrm{MHz}, \mathrm{CDCl}_{3}\right): \delta$ $8.41(\mathrm{~d}, J=4.5 \mathrm{~Hz}, 1 \mathrm{H}), 7.42-7.37(\mathrm{~m}, 1 \mathrm{H}), 7.17(\mathrm{~d}, J=8.0 \mathrm{~Hz}, 1 \mathrm{H}), 7.10(\mathrm{~d}, J=5.5 \mathrm{~Hz}, 2 \mathrm{H}), 7.05-6.95(\mathrm{~m}, 3 \mathrm{H}), 6.87$ (t, $J=8.0 \mathrm{~Hz}, 1 \mathrm{H}$ ), 5.72 (ddd, $J=32.8,9.2,4.4 \mathrm{~Hz}, 1 \mathrm{H}), 4.60(\mathrm{dd}, J=18.6,9.3 \mathrm{~Hz}, 1 \mathrm{H}), 4.31-4.24(\mathrm{~m}, 2 \mathrm{H}), 3.93(\mathrm{dd}, J$ $=18.6,4.4 \mathrm{~Hz}, 1 \mathrm{H}), 3.88(\mathrm{~s}, 3 \mathrm{H}), 1.22(\mathrm{t}, J=7.1 \mathrm{~Hz}, 3 \mathrm{H}) .{ }^{19} \mathrm{~F}$ NMR $\left(376 \mathrm{MHz}, \mathrm{CDCl}_{3}\right) \delta-163.29(\mathrm{~d}, J=33.0 \mathrm{~Hz}) .{ }^{13} \mathrm{C} \mathrm{NMR}$ (125 MHz, CDCl 3 ): $\delta 189.6$ (s), 168.6 (d, $J=23.9 \mathrm{~Hz}$ ), 156.3 (d, J=28.3 Hz), 148.5 (s), 142.6 (s), 138.8 (s), 136.0 (s), 135.6 (s), 134.2 (s), 130.1 (s), 128.9 (s), 128.4 (d, J=6.7 Hz), 128.2 (s), 126.9 (s), 123.2 (s), 119.9 (d, $J=7.4$ Hz), 100.3 (d, $J=202.5 \mathrm{~Hz}$ ), 62.5 (s), 42.4 (d, $J=21.4 \mathrm{~Hz}$ ), 38.5 (s), 36.2 (s), 14.0 (s). HRMS (ESI, m/z): [M + H] calculated for $\mathrm{C}_{22} \mathrm{H}_{21} \mathrm{Cl}_{2} \mathrm{FN}_{3} \mathrm{O}_{3}$ : 464.0939; found: 464.0949. [a] $]^{23}=-203.7$ (c = 0.95, $\mathrm{CHCl}_{3}, 91 \%$ ee, >20:1 dr); HPLC (IB3, IPA/nhexane $=10 / 90$, flow rate $=1 \mathrm{~mL} / \mathrm{min}, \mathrm{I}=254 \mathrm{~nm}$ ) $\mathrm{t}_{\mathrm{R}}=15.90 \mathrm{~min}$ (minor), $22.08 \mathrm{~min}$ (major).

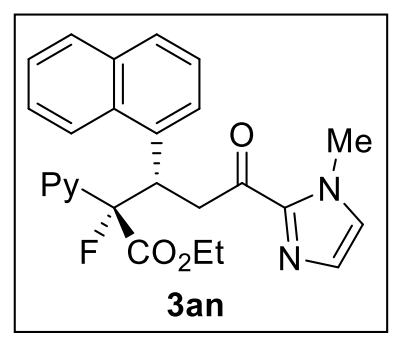

Ethyl (2S,3S)-2-fluoro-5-(1-methyl-1H-imidazol-2-yl)-3-(naphthalen-1-yl)-5-oxo-2-(pyridin-2-yl)pentanoate (3an): The compound was prepared following the synthetic procedure A from pyridinyl fluoroacetate 1a as limiting starting material $(36 \mathrm{mg}, 0.20 \mathrm{mmol})$. The desired product was isolated by silica gel column chromatography $(30: 70=$ EtOAc/petroleum ether, v/v) to afford a light yellow gel (84 mg, 95\% yield); ${ }^{1} \mathrm{H}$ NMR $\left(400 \mathrm{MHz}, \mathrm{CDCl}_{3}\right): \delta 8.54(\mathrm{~d}, J=4.7$ $\mathrm{Hz}, 1 \mathrm{H}), 7.69-7.55(\mathrm{~m}, 3 \mathrm{H}), 7.49(\mathrm{~d}, J=8.5 \mathrm{~Hz}, 1 \mathrm{H}), 7.35-7.25(\mathrm{~m}, 4 \mathrm{H}), 7.09(\mathrm{dd}, J=5.5,2.0 \mathrm{~Hz}, 2 \mathrm{H}), 7.01-6.93(\mathrm{~m}$, $1 \mathrm{H}), 6.89$ (s, 1H), 4.97 (ddd, $J=34.6,11.0,2.9 \mathrm{~Hz}, 1 \mathrm{H}$ ), 4.39 (dd, $J=17.8,11.1 \mathrm{~Hz}, 1 \mathrm{H}$ ), $4.28(\mathrm{q}, J=7.1 \mathrm{~Hz}, 2 \mathrm{H}), 3.76$ (s, 3H), 3.56 (dd, $J=17.8,3.0 \mathrm{~Hz}, 1 \mathrm{H}), 1.25$ (t, $J=7.1 \mathrm{~Hz}, 3 \mathrm{H}) .{ }^{19} \mathrm{~F}$ NMR $\left(376 \mathrm{MHz}, \mathrm{CDCl}_{3}\right) \delta-175.14(\mathrm{~d}, J=34.5 \mathrm{~Hz})$. ${ }^{13} \mathrm{C}$ NMR (125 MHz, $\mathrm{CDCl}_{3}$ ): $\delta 189.3$ (s), 168.7 (d, J = 24.8 Hz), 156.3 (d, J = 27.8 Hz), 148.7 (s), 142.9 (s), 136.5 (s), 135.7 (s), 133.0 (s), 132.3 (s), 129.0 (s), 128.7 (s), 128.2 (s), 128.0 (s), 127.4 (s), 127.1 (s), 126.9 (s), 125.5 (d, J = 2.5 Hz), 122.9 (s), 120.2 (d, J = 9.4 Hz), 100.2 (d, J=196.0 Hz), 62.4 (s), 46.2 (d, J=18.9 Hz), 40.3 (d, J = 2.6 Hz), 36.0 (s), 14.1 (s). HRMS (ESI, m/z): $[\mathrm{M}+\mathrm{H}]^{+}$calculated for $\mathrm{C}_{26} \mathrm{H}_{25} \mathrm{FN}_{3} \mathrm{O}_{3}: 446.1874$; found: 446.1882. [ $\left.\mathrm{\alpha}\right]_{\mathrm{D}}{ }^{23}=-163.4(\mathrm{c}=2.53$, $\mathrm{CHCl}_{3}, 99 \%$ ee, $>20: 1 \mathrm{dr}$ ); HPLC (IA3, IPA $/ \mathrm{n}$-hexane $=30 / 70$, flow rate $\left.=0.8 \mathrm{~mL} / \mathrm{min}, \mathrm{I}=254 \mathrm{~nm}\right) \mathrm{t}_{\mathrm{R}}=20.33 \mathrm{~min}$ (minor), $41.72 \mathrm{~min}$ (major). 


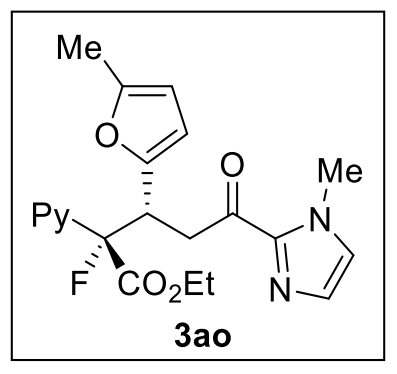

Ethyl (2S,3S)-2-fluoro-5-(1-methyl-1H-imidazol-2-yl)-3-(5-methylfuran-2-yl)-5-oxo-2-(pyridin-2-yl)pentanoate (3ao): The compound was prepared following the synthetic procedure $A$ from pyridinyl fluoroacetate 1a as limiting starting material $(36 \mathrm{mg}, 0.20 \mathrm{mmol})$. The desired product was isolated by silica gel column chromatography $(30: 70=$ EtOAc/petroleum ether, v/v) to afford a light yellow gel (77 mg, 96\% yield); ${ }^{1} \mathrm{H}$ NMR $\left(400 \mathrm{MHz}, \mathrm{CDCl}_{3}\right): \delta 8.52(\mathrm{~d}, J=4.8$ $\mathrm{Hz}, 1 \mathrm{H}), 7.50$ (td, $J=7.8,1.7 \mathrm{~Hz}, 1 \mathrm{H}), 7.24-7.19(\mathrm{~m}, 1 \mathrm{H}), 7.15-7.00(\mathrm{~m}, 2 \mathrm{H}), 6.92(\mathrm{~s}, 1 \mathrm{H}), 5.72(\mathrm{~d}, J=3.0 \mathrm{~Hz}, 1 \mathrm{H})$, $5.54(\mathrm{~d}, J=2.3 \mathrm{~Hz}, 1 \mathrm{H}$ ), 4.75 (ddd, $J=31.4,10.9,2.9 \mathrm{~Hz}, 1 \mathrm{H}$ ), 4.20 (q, $J=7.2 \mathrm{~Hz}, 2 \mathrm{H}$ ), 4.09 (dd, $J=17.9,10.9 \mathrm{~Hz}, 1 \mathrm{H}$ ), $3.85(\mathrm{~s}, 3 \mathrm{H}), 3.34(\mathrm{dd}, J=17.9,2.8 \mathrm{~Hz}, 1 \mathrm{H}), 1.93(\mathrm{~s}, 3 \mathrm{H}), 1.17(\mathrm{t}, J=7.1 \mathrm{~Hz}, 3 \mathrm{H}) .{ }^{19} \mathrm{~F} \mathrm{NMR}\left(376 \mathrm{MHz}, \mathrm{CDCl}_{3}\right) \delta-172.10$ (d, $J=31.9 \mathrm{~Hz}$ ). ${ }^{13} \mathrm{C}$ NMR (100 MHz, $\mathrm{CDCl}_{3}$ ): $\delta 189.2$ (s), 168.3 (d, J = 24.5 Hz), 156.5 (d, J = 27.2 Hz), 150.9 (s), 149.6 (s), 148.7 (s), 142.9 (s), 136.4 (s), 129.1 (s), 126.9 (s), 123.1 (s), 120.1 (d, J = 8.9 Hz), 109.1 (s), 105.9 (s), 99.1 (d, J = $196.0 \mathrm{~Hz}$ ), 62.3 (s), 40.6 (d, $J=20.7 \mathrm{~Hz}$ ), 38.3 (s), 36.1 (s), 14.1 (s), 13.4 (s). HRMS (ESI, m/z): [M + H] calculated for

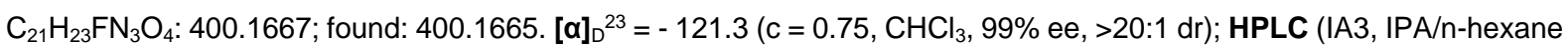
$=20 / 80$, flow rate $=0.9 \mathrm{~mL} / \mathrm{min}, \mathrm{I}=254 \mathrm{~nm}$ ) $t_{R}=17.64 \mathrm{~min}$ (minor), $26.15 \mathrm{~min}$ (major).

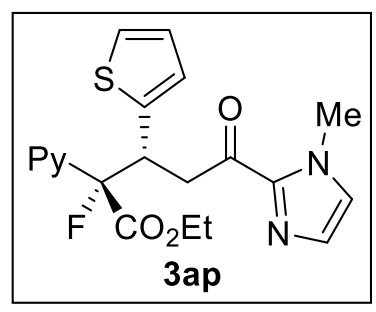

Ethyl (2S,3R)-2-fluoro-5-(1-methyl-1H-imidazol-2-yl)-5-oxo-2-(pyridin-2-yl)-3-(thiophen-2-yl)pentanoate (3ap): The compound was prepared following the synthetic procedure $A$ from pyridinyl fluoroacetate 1a as limiting starting material (36 mg, $0.20 \mathrm{mmol})$. The desired product was isolated by silica gel column chromatography (30:70 = EtOAc/petroleum ether, v/v) to afford a light yellow gel $\left(76 \mathrm{mg}, 95 \%\right.$ yield); ${ }^{1} \mathrm{H}$ NMR $\left(400 \mathrm{MHz}, \mathrm{CDCl}_{3}\right): \delta 8.51$ (d, J=4.0 Hz, $\left.1 \mathrm{H}\right), 7.44$ (td, $J=7.8,1.6 \mathrm{~Hz}, 1 \mathrm{H}), 7.20(\mathrm{~d}, J=6.1 \mathrm{~Hz}, 1 \mathrm{H}), 7.13-6.99(\mathrm{~m}, 2 \mathrm{H}), 6.90(\mathrm{~s}, 1 \mathrm{H}), 6.86(\mathrm{~d}, J=5.2 \mathrm{~Hz}, 1 \mathrm{H}), 6.68(\mathrm{~d}, J=3.4$ $\mathrm{Hz}, 1 \mathrm{H}), 6.59$ (dd, $J=4.9,3.7 \mathrm{~Hz}, 1 \mathrm{H}), 5.06$ (ddd, $J=33.3,11.0,2.7 \mathrm{~Hz}, 1 \mathrm{H}), 4.24-4.08(\mathrm{~m}, 3 \mathrm{H}), 3.80(\mathrm{~s}, 3 \mathrm{H}), 3.40(\mathrm{dd}$, $J=17.6,2.7 \mathrm{~Hz}, 1 \mathrm{H}), 1.17(\mathrm{t}, J=7.1 \mathrm{~Hz}, 3 \mathrm{H}) .{ }^{19} \mathrm{~F}$ NMR $\left(376 \mathrm{MHz}, \mathrm{CDCl}_{3}\right) \delta-174.62(\mathrm{~d}, J=33.6 \mathrm{~Hz}) .{ }^{13} \mathrm{C} \mathrm{NMR}(100 \mathrm{MHz}$, $\mathrm{CDCl}_{3}$ ): $\delta 188.8$ (s), 168.3 (d, $J=24.9 \mathrm{~Hz}$ ), 156.1 (d, $\left.J=27.7 \mathrm{~Hz}\right), 148.9$ (d, $J=2.4 \mathrm{~Hz}$ ), 142.9 (s), 140.2 (s), 136.6 (s), 129.1 (s), 127.1 (s), 127.0 (s), 126.1 (s), 124.5 (s), 123.1 (s), 120.2 (d, J = 9.3 Hz), 99.5 (d, J = 195.9 Hz), 62.5 (s), 41.8 (d, $J=20.0 \mathrm{~Hz}$ ), 41.4 (d, $J=3.0 \mathrm{~Hz}$ ), 36.1 (s), 14.1 (s). HRMS (ESI, m/z): [M + H] calculated for $\mathrm{C}_{20} \mathrm{H}_{21} \mathrm{FN}_{3} \mathrm{O}_{3} \mathrm{~S}: 402.1282$; found: 402.1283. [a $]_{D}^{23}=-125.4\left(\mathrm{c}=3, \mathrm{CHCl}_{3}, 96 \%\right.$ ee, $>20: 1 \mathrm{dr}$ ); HPLC (IA3, IPA/n-hexane = 20/80, flow rate $=0.9$ $\mathrm{mL} / \mathrm{min}, \mathrm{I}=254 \mathrm{~nm}$ ) $\mathrm{t}_{\mathrm{R}}=23.60 \mathrm{~min}$ (minor), $35.92 \mathrm{~min}$ (major). 


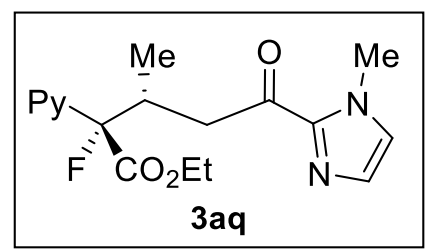

Ethyl (2S,3R)-2-fluoro-3-methyl-5-(1-methyl-1H-imidazol-2-yl)-5-oxo-2-(pyridin-2-yl)pentanoate (3aq): The compound was prepared following the synthetic procedure A from pyridinyl fluoroacetate 1a as limiting starting material (36 mg, $0.20 \mathrm{mmol})$. The desired product was isolated by silica gel column chromatography (30:70 = EtOAc/petroleum ether, v/v) to afford a light yellow gel (54 mg, 81\% yield); ${ }^{1} \mathbf{H}$ NMR $\left(400 \mathrm{MHz}, \mathrm{CDCl}_{3}\right):{ }^{1} \mathbf{H}$ NMR $(500 \mathrm{MHz}, \mathrm{CHLOROFORM-}$ D) $\delta 8.64-8.57(\mathrm{~m}, 1 \mathrm{H}), 7.74-7.65(\mathrm{~m}, 1 \mathrm{H}), 7.59(\mathrm{~d}, J=8.0 \mathrm{~Hz}, 1 \mathrm{H}), 7.24-7.15(\mathrm{~m}, 1 \mathrm{H}), 7.08(\mathrm{~s}, 1 \mathrm{H}), 7.00(\mathrm{~s}, 1 \mathrm{H})$, 4.17 (q, $J=7.1 \mathrm{~Hz}, 2 \mathrm{H}$ ), $3.96(\mathrm{~s}, 3 \mathrm{H}), 3.68-3.54(\mathrm{~m}, 1 \mathrm{H}), 3.41$ (dd, $J=17.0,10.0 \mathrm{~Hz}, 1 \mathrm{H}), 3.16(\mathrm{dd}, J=17.0,2.8 \mathrm{~Hz}$, $1 \mathrm{H}), 1.17$ (t, $J=7.1 \mathrm{~Hz}, 3 \mathrm{H}), 0.78(\mathrm{~d}, J=6.8 \mathrm{~Hz}, 3 \mathrm{H}) .{ }^{19} \mathrm{~F}$ NMR (376 MHz, CHLOROFORM-D) $\delta-177.12$ (d, $J=33.2 \mathrm{~Hz}$ ). ${ }^{13} \mathrm{C}$ NMR (125 MHz, CHLOROFORM-D) $\delta 190.9$ (s), 168.9 (d, $\left.J=25.9 \mathrm{~Hz}\right), 156.5$ (d, $\left.J=26.8 \mathrm{~Hz}\right), 149.3$ (s), 143.2 (s), 136.8 (s), 129.1 (s), 127.0 (s), 123.2 (s), 120.4 (d, J = 9.5 Hz), 100.3 (d, J = $192.8 \mathrm{~Hz}$ ), 62.0 (s), 40.8 (s), 36.2 (s), 35.0 (d, $J=20.4 \mathrm{~Hz}$ ), 14.0 (d, $J=9.0 \mathrm{~Hz}$ ). HRMS (ESI, m/z): [M + H]+ calculated for $\mathrm{C}_{17} \mathrm{H}_{21} \mathrm{FN}_{3} \mathrm{O}_{3}: 334.1561$; found: 334.1580 . $[\alpha]_{D}^{23}=-66.9\left(\mathrm{c}=1, \mathrm{CHCl}_{3}, 99 \%\right.$ ee, $\left.4: 1 \mathrm{dr}\right) ; \mathrm{HPLC}(\mathrm{ID}, \mathrm{IPA} / \mathrm{n}$-hexane $=30 / 70$, flow rate $=1.0 \mathrm{~mL} / \mathrm{min}, \mathrm{I}=254 \mathrm{~nm}) \mathrm{t}_{\mathrm{R}}=$ $15.7 \mathrm{~min}$ (minor), $21.1 \mathrm{~min}$ (major).

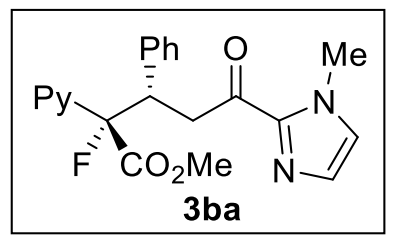

Methyl (2S,3S)-2-fluoro-5-(1-methyl-1H-imidazol-2-yl)-5-oxo-3-phenyl-2-(pyridin-2-yl)pentanoate (3ba): The compound was prepared following the synthetic procedure A from methyl 2-fluoro-2-(pyridin-2-yl)acetate (16) as limiting starting material $(34 \mathrm{mg}, 0.20 \mathrm{mmol})$. The desired product was isolated by silica gel column chromatography $(40: 60=$ EtOAc/petroleum ether, v/v) to afford a light yellow gel (39 mg, $51 \%$ yield); $\mathbf{m p ~} 111-114{ }^{\circ} \mathrm{C} ;{ }^{1} \mathbf{H ~} \mathbf{~ N M R}\left(500 \mathrm{MHz}, \mathrm{CDCl}_{3}\right)$ $\delta 8.47(\mathrm{~d}, J=4.5 \mathrm{~Hz}, 1 \mathrm{H}), 7.35-7.29(\mathrm{~m}, 1 \mathrm{H}), 7.09-7.04(\mathrm{~m}, 2 \mathrm{H}), 7.04-6.99(\mathrm{~m}, 2 \mathrm{H}), 6.98-6.88(\mathrm{~m}, 4 \mathrm{H}), 6.86(\mathrm{~s}$, $1 \mathrm{H}$ ), 4.69 (ddd, $J=34.7,10.8,2.9 \mathrm{~Hz}, 1 \mathrm{H}$ ), 4.17 (dd, $J=17.7,10.8 \mathrm{~Hz}, 1 \mathrm{H}$ ), $3.73(\mathrm{~s}, 3 \mathrm{H}$ ), 3.71 (s, $3 \mathrm{H}$ ), 3.41 (ddd, $J=12.2$, 7.2, $1.5 \mathrm{~Hz}, 1 \mathrm{H}) .{ }^{19} \mathrm{~F}$ NMR $\left(376 \mathrm{MHz}, \mathrm{CDCl}_{3}\right) \delta-175.37$ (d, J = 34.7 Hz). ${ }^{13} \mathrm{C}$ NMR $\left(126 \mathrm{MHz}, \mathrm{CDCl}_{3}\right) \delta 189.2(\mathrm{~s}), 169.1$ (d, $J=25.1 \mathrm{~Hz}$ ), 156.2 (d, $J=28.0 \mathrm{~Hz}$ ), 148.8 (s), 142.9 (s), 137.8 (s), 136.5 (s), 129.8 (s), 128.9 (s), 128.4 (s), 127.7 (s), 126.9 (d, $J=17.2 \mathrm{~Hz}$ ), 123.0 (s), 120.2 (d, $J=9.4 \mathrm{~Hz}$ ), 100.2 (d, $J=195.8 \mathrm{~Hz}$ ), 53.2 (s), 46.4 (d, J = 18.8 Hz), 40.2 (s),

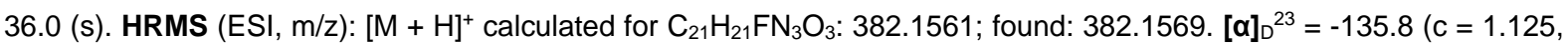
$\mathrm{CHCl}_{3}, 94 \%$ ee, $2: 1 \mathrm{dr}$ ); HPLC (ID, IPA $/ \mathrm{n}$-hexane $=24 / 76$, flow rate $\left.=1.0 \mathrm{~mL} / \mathrm{min}, \mathrm{I}=254 \mathrm{~nm}\right) \mathrm{t}_{\mathrm{R}}=41.91 \mathrm{~min}$ (minor), $79.52 \mathrm{~min}$ (major).

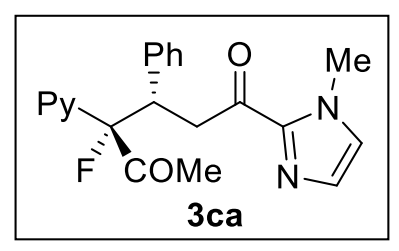

(3S,4S)-4-Fluoro-1-(1-methyl-1H-imidazol-2-yl)-3-phenyl-4-(pyridin-2-yl)hexane-1,5-dione (3ca): The compound was prepared following the synthetic procedure A from 1-fluoro-1-(pyridin-2-yl)propan-2-one (1c) as limiting starting material $(31 \mathrm{mg}, 0.20 \mathrm{mmol})$. The desired product was isolated by silica gel column chromatography $(30: 60=$ 
EtOAc/petroleum ether, v/v) to afford a light yellow gel (40 mg, $54 \%$ yield); ${ }^{1} \mathrm{H}$ NMR $\left(400 \mathrm{MHz}, \mathrm{CDCl}_{3}\right) \delta 8.61$ (d, $J=4.4$ $\mathrm{Hz}, 1 \mathrm{H}), 7.72-7.58(\mathrm{~m}, 2 \mathrm{H}), 7.47-7.39(\mathrm{~m}, 2 \mathrm{H}), 7.24-7.08(\mathrm{~m}, 4 \mathrm{H}), 6.94(\mathrm{~s}, 1 \mathrm{H}), 6.80(\mathrm{~s}, 1 \mathrm{H}), 4.85$ (ddd, $J=35.9$, 11.1, 3.1 Hz, 1H), 3.93 (dd, $J=17.7,11.2 \mathrm{~Hz}, 1 \mathrm{H}$ ), 3.69 (s, 3H), 2.81 (dd, $J=17.7,3.1 \mathrm{~Hz}, 1 \mathrm{H}), 1.82(\mathrm{~d}, J=5.0 \mathrm{~Hz}, 3 \mathrm{H}$ ). ${ }^{19} \mathrm{~F}$ NMR $\left(376 \mathrm{MHz}, \mathrm{CDCl}_{3}\right) \delta-173.89$ (d, $\left.J=34.9 \mathrm{~Hz}\right) .{ }^{13} \mathrm{C}$ NMR $\left(126 \mathrm{MHz}, \mathrm{CDCl}_{3}\right) \delta 204.6(\mathrm{~d}, J=29.1 \mathrm{~Hz}), 189.5(\mathrm{~s})$, 155.1 (d, $J=27.0 \mathrm{~Hz}$ ), 149.8 (s), 142.9 (s), 138.2 (s), 137.1 (s), 130.2 (s), 128.9 (s), 128.3 (s), 127.3 (s), 126.8 (s), 123.4 (s), 120.9 (d, $J=10.1 \mathrm{~Hz}), 105.5$ (d, $J=195.1 \mathrm{~Hz}), 45.6$ (d, $J=17.6 \mathrm{~Hz}), 39.5$ (s), 36.0 (s), 26.4 (s). HRMS (ESI, m/z): [M $+\mathrm{H}^{+}$calculated for $\mathrm{C}_{21} \mathrm{H}_{21} \mathrm{FN}_{3} \mathrm{O}_{2}$ : 366.1612; found: 366.1622. [ $\left.\alpha\right]_{D}^{23}=-49.6$ (c = 1.4, $\mathrm{CHCl}_{3}, 64 \%$ ee, 4:1 dr); HPLC (ID, $\mathrm{IPA} / \mathrm{n}$-hexane $=30 / 70$, flow rate $=1 \mathrm{~mL} / \mathrm{min}, \mathrm{I}=254 \mathrm{~nm}$ ) $\mathrm{t}_{\mathrm{R}}=19.16 \mathrm{~min}$ (major), $21.43 \mathrm{~min}$ (minor).

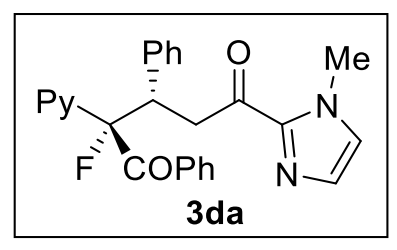

(2S,3S)-2-Fluoro-5-(1-methyl-1H-imidazol-2-yl)-1,3-diphenyl-2-(pyridin-2-yl)pentane-1,5-dione (3da): The compound was prepared following the synthetic procedure A from 2-fluoro-1-phenyl-2-(pyridin-2-yl)ethan-1-one (1d) as limiting starting material $(43 \mathrm{mg}, 0.20 \mathrm{mmol}$ ). The desired product was isolated by silica gel column chromatography $\left(40: 60=\right.$ EtOAc/petroleum ether, v/v) to afford a light yellow gel $\left(37 \mathrm{mg}, 43 \%\right.$ yield); ${ }^{1} \mathbf{H}$ NMR $\left(500 \mathrm{MHz}, \mathrm{CDCl}_{3}\right): \delta 8.62$ (d, $J=4.3 \mathrm{~Hz}, 1 \mathrm{H}), 7.77$ (d, $J=7.9 \mathrm{~Hz}, 1 \mathrm{H}), 7.72-7.66(\mathrm{~m}, 1 \mathrm{H}), 7.48(\mathrm{~d}, J=8.1 \mathrm{~Hz}, 2 \mathrm{H}), 7.40(\mathrm{~d}, J=7.9 \mathrm{~Hz}, 2 \mathrm{H}), 7.29$ (t, $J=7.4 \mathrm{~Hz}, 1 \mathrm{H}), 7.20-7.07(\mathrm{~m}, 5 \mathrm{H}), 7.02(\mathrm{dd}, J=13.9,6.5 \mathrm{~Hz}, 2 \mathrm{H}), 6.84(\mathrm{~s}, 1 \mathrm{H}), 4.99$ (ddd, $J=34.6,10.9,3.1 \mathrm{~Hz}$, $1 \mathrm{H}$ ), 4.00 (dd, $J=17.6,11.0 \mathrm{~Hz}, 1 \mathrm{H}$ ), $3.71(\mathrm{~s}, 3 \mathrm{H}), 3.00$ (dd, $J=17.7,3.2 \mathrm{~Hz}, 1 \mathrm{H}) .{ }^{19} \mathrm{~F} \mathrm{NMR}\left(376 \mathrm{MHz}, \mathrm{CDCl}_{3}\right) \delta-168.91$ (d, $J=30.4 \mathrm{~Hz}$ ). ${ }^{13} \mathrm{C}$ NMR (125 MHz, $\left.\mathrm{CDCl}_{3}\right): \delta 196.6$ (d, $\left.J=27.5 \mathrm{~Hz}\right), 189.2$ (s), 155.9 (d, $\left.J=26.5 \mathrm{~Hz}\right), 149.8$ (s), 142.2 (s), 138.5 (s), 137.3 (s), 135.8 (d, J = 2.2 Hz), 132.5 (s), 130.6 (s), 129.4 (s), 129.3 (d, J = 6.0 Hz), 129.0 (s), 128.0 (d, J $=10.6 \mathrm{~Hz}$ ), 127.1 (s), 126.8 (s), 123.4 (s), 121.4 (d, $J=8.5 \mathrm{~Hz}$ ), 105.8 (d, J=196.9 Hz), 46.6 (d, J = 18.6 Hz), 39.9 (s),

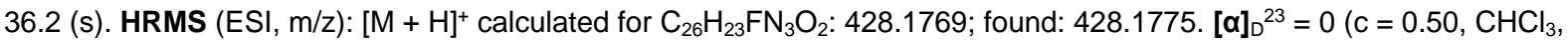
$0 \%$ ee, $>20: 1 \mathrm{dr}$ ); HPLC (IA3, IPA $/ \mathrm{n}$-hexane $=30 / 70$, flow rate $=0.8 \mathrm{~mL} / \mathrm{min}, \mathrm{I}=254 \mathrm{~nm}$ ) $\mathrm{t}_{\mathrm{R}}=13.40 \mathrm{~min}$ (major), 19.58 $\min$ (minor).

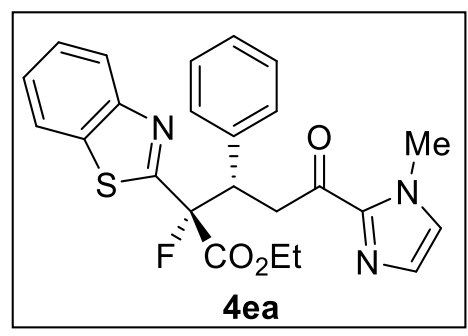

Ethyl (2R,3S)-2-(benzo[d]thiazol-2-yl)-2-fluoro-5-(1-methyl-1H-imidazol-2-yl)-5-oxo-3-phenylpentanoate (4ea): The compound was prepared following the synthetic procedure B from benzothiazolyl fluoroacetate 1e as limiting starting material $(48 \mathrm{mg}, 0.20 \mathrm{mmol})$. The desired product was isolated by silica gel column chromatography $(20: 80=$ EtOAc/petroleum ether, v/v) to afford a light yellow gel (83 mg, 92\% yield); $\mathbf{m p} 130-134{ }^{\circ} \mathrm{C} ;{ }^{1} \mathbf{H} \mathbf{~ N M R}\left(500 \mathrm{MHz}, \mathrm{CDCl}_{3}\right)$ : $\delta 8.03(\mathrm{~d}, J=8.3 \mathrm{~Hz}, 1 \mathrm{H}), 7.69(\mathrm{~d}, J=8.0 \mathrm{~Hz}, 1 \mathrm{H}), 7.45-7.36(\mathrm{~m}, 1 \mathrm{H}), 7.29(\mathrm{dd}, J=11.7,4.5 \mathrm{~Hz}, 3 \mathrm{H}), 7.09(\mathrm{~s}, 1 \mathrm{H}), 7.06$ - $6.98(\mathrm{~m}, 3 \mathrm{H}), 6.93(\mathrm{~s}, 1 \mathrm{H}), 4.82(\mathrm{ddd}, J=33.9,10.7,3.2 \mathrm{~Hz}, 1 \mathrm{H}), 4.33-4.23(\mathrm{~m}, 3 \mathrm{H}), 3.78(\mathrm{~s}, 3 \mathrm{H}), 3.48(\mathrm{dd}, J=17.6$, $3.3 \mathrm{~Hz}, 1 \mathrm{H}), 1.27$ (t, $J=7.1 \mathrm{~Hz}, 3 \mathrm{H}) .{ }^{19} \mathrm{~F} \mathrm{NMR}\left(376 \mathrm{MHz}, \mathrm{CDCl}_{3}\right) \delta-162.91$ (d, J=34.7 Hz). ${ }^{13} \mathrm{C} \mathrm{NMR}\left(125 \mathrm{MHz}, \mathrm{CDCl}_{3}\right)$ : $\delta 188.5$ (s), 167.0 (s), 166.7 (d, $J=8.4$ Hz), 153.0 (s), 142.4 (s), 136.8 (s), 135.0 (s), 130.0 (s), 128.5 (s), 128.1 (s), 127.4 
(s), 127.0 (s), 126.1 (s), 125.4 (s), 123.8 (s), 121.6 (s), 99.2 (d, J = $195.7 \mathrm{~Hz}$ ), 63.2 (s), 47.3 (d, J = 19.1 Hz), 40.2 (s),

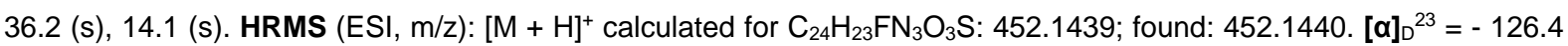
( $\mathrm{C}=1.45, \mathrm{CHCl}_{3}, 98 \%$ ee, $\left.>20: 1 \mathrm{dr}\right) ; \mathrm{HPLC}(\mathrm{IA} 3, \mathrm{IPA} / \mathrm{n}$-hexane $=30 / 70$, flow rate $=0.8 \mathrm{~mL} / \mathrm{min}, \mathrm{I}=254 \mathrm{~nm}) \mathrm{t}_{\mathrm{R}}=16.62$ $\min$ (major), $11.39 \mathrm{~min}$ (minor).

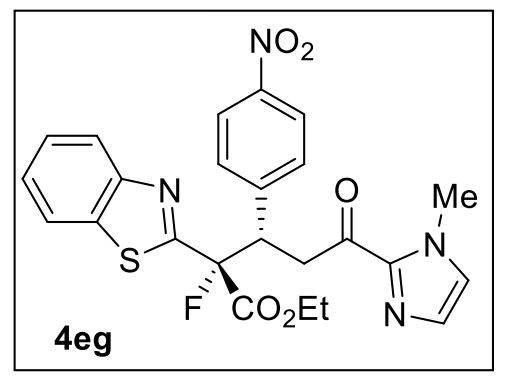

Ethyl (2R,3S)-2-(benzo[d]thiazol-2-yl)-2-fluoro-5-(1-methyl-1H-imidazol-2-yl)-3-(4-nitrophenyl)-5-oxopentanoate (4eg): The compound was prepared following the synthetic procedure B from benzothiazolyl fluoroacetate 1e as limiting starting material $(48 \mathrm{mg}, 0.20 \mathrm{mmol})$. The desired product was isolated by silica gel column chromatography $(20: 80=$ EtOAc/petroleum ether, v/v) to afford a light yellow gel (90 mg, $91 \%$ yield); ${ }^{1} \mathbf{H}$ NMR $\left(500 \mathrm{MHz}, \mathrm{CDCl}_{3}\right): \delta 8.04(\mathrm{~d}, J=8.2$ $\mathrm{Hz}, 1 \mathrm{H}), 7.90(\mathrm{~d}, J=8.1 \mathrm{~Hz}, 2 \mathrm{H}), 7.74(\mathrm{~d}, J=8.0 \mathrm{~Hz}, 1 \mathrm{H}), 7.51(\mathrm{~d}, J=8.2 \mathrm{~Hz}, 2 \mathrm{H}), 7.45(\mathrm{t}, J=7.6 \mathrm{~Hz}, 1 \mathrm{H}), 7.34(\mathrm{t}, J=$ $7.5 \mathrm{~Hz}, 1 \mathrm{H}), 7.15(\mathrm{~s}, 1 \mathrm{H}), 7.01(\mathrm{~s}, 1 \mathrm{H}), 5.00(\mathrm{dd}, J=33.4,6.3 \mathrm{~Hz}, 1 \mathrm{H}), 4.33(\mathrm{~d}, J=6.5 \mathrm{~Hz}, 3 \mathrm{H}), 3.84(\mathrm{~s}, 3 \mathrm{H}), 3.54(\mathrm{~d}, J=$ $9.2 \mathrm{~Hz}, 1 \mathrm{H}), 1.29(\mathrm{t}, J=6.3 \mathrm{~Hz}, 3 \mathrm{H}) .{ }^{19} \mathrm{~F} \mathrm{NMR}\left(376 \mathrm{MHz}, \mathrm{CDCl}_{3}\right) \delta-163.83(\mathrm{~d}, J=32.9 \mathrm{~Hz}) .{ }^{13} \mathrm{C} \mathrm{NMR}\left(125 \mathrm{MHz}, \mathrm{CDCl}_{3}\right)$ : $\delta 188.0$ (s), 166.3 (d, $J=25.2 \mathrm{~Hz}$ ), 165.8 (d, J = 32.4 Hz), 152.9 (s), 147.1 (s), 144.8 (s), 134.8 (s), 131.1 (s), 129.3 (s), 128.4 (s), 127.7 (s), 126.5 (s), 125.9 (s), 123.8 (s), 123.2 (s), 121.8 (s), 99.0 (d, J=196.1 Hz), 63.6 (s), 46.9 (d, J = 18.6 $\mathrm{Hz}$ ), 39.7 (s), 36.2 (s), 14.1 (s). HRMS (ESI, m/z): [M + H] calculated for $\mathrm{C}_{24} \mathrm{H}_{22} \mathrm{FN}_{4} \mathrm{O}_{5} \mathrm{~S}: 497.1289$; found: 497.1294. $[\alpha]_{D}^{23}=-174.4\left(\mathrm{c}=2.8, \mathrm{CHCl}_{3}, 99 \%\right.$ ee, $\left.>20: 1 \mathrm{dr}\right) ; \mathrm{HPLC}(\mathrm{IA} 3, \mathrm{IPA} / \mathrm{n}$-hexane $=30 / 70$, flow rate $=0.8 \mathrm{~mL} / \mathrm{min}, \mathrm{I}=254$ $\mathrm{nm}) \mathrm{t}_{\mathrm{R}}=37.40 \mathrm{~min}$ (major), $16.54 \mathrm{~min}$ (minor).<smiles>CCO[C@H](c1nc2ccccc2s1)[C@](CCC(=O)c1nccn1C)(OCC)c1ccc(C)cc1</smiles>

Ethyl (2R,3S)-2-(benzo[d]thiazol-2-yl)-2-fluoro-5-(1-methyl-1H-imidazol-2-yl)-5-oxo-3-(p-tolyl)pentanoate (4eb): The compound was prepared following the synthetic procedure B from benzothiazolyl fluoroacetate 1e as limiting starting material $(48 \mathrm{mg}, 0.20 \mathrm{mmol})$. The desired product was isolated by silica gel column chromatography $(20: 80=$ EtOAc/petroleum ether, v/v) to afford a light yellow gel (83 mg, 89\% yield); ${ }^{1} \mathrm{H}$ NMR $\left(500 \mathrm{MHz}, \mathrm{CDCl}_{3}\right): \delta 8.04(\mathrm{~d}, J=8.2$ $\mathrm{Hz}, 1 \mathrm{H}), 7.72(\mathrm{~d}, J=8.0 \mathrm{~Hz}, 1 \mathrm{H}), 7.42(\mathrm{t}, J=7.6 \mathrm{~Hz}, 1 \mathrm{H}), 7.35-7.27(\mathrm{~m}, 1 \mathrm{H}), 7.17(\mathrm{~d}, J=7.6 \mathrm{~Hz}, 2 \mathrm{H}), 7.11(\mathrm{~s}, 1 \mathrm{H}), 6.94$ $(\mathrm{s}, 1 \mathrm{H}), 6.85(\mathrm{~d}, J=7.7 \mathrm{~Hz}, 2 \mathrm{H}), 4.78(\mathrm{dd}, J=33.8,9.8 \mathrm{~Hz}, 1 \mathrm{H}), 4.35-4.18(\mathrm{~m}, 3 \mathrm{H}), 3.80(\mathrm{~s}, 3 \mathrm{H}), 3.47(\mathrm{~d}, J=16.4 \mathrm{~Hz}$, $1 \mathrm{H}), 2.11(\mathrm{~s}, 3 \mathrm{H}), 1.28(\mathrm{t}, J=6.4 \mathrm{~Hz}, 3 \mathrm{H}) .{ }^{19} \mathrm{~F} \mathrm{NMR}\left(376 \mathrm{MHz}, \mathrm{CDCl}_{3}\right) \delta-162.60(\mathrm{~d}, J=32.5 \mathrm{~Hz}) .{ }^{13} \mathrm{C} \mathrm{NMR}(125 \mathrm{MHz}$, $\mathrm{CDCl}_{3}$ ): $\delta 188.8$ (s), 167.1 (d, J = 8.8 Hz), 166.8 (d, J=15.7 Hz), 152.9 (s), 142.7 (s), 136.8 (s), 135.1 (s), 133.8 (s), 129.8 
(s), 128.8 (s), 128.4 (s), 127.1 (s), 126.1 (s), 125.4 (s), 123.8 (s), 121.1 (s), 99.5 (d, J = $195.2 \mathrm{~Hz}), 63.2$ (s), 46.9 (d, $J=$ $19.1 \mathrm{~Hz}$ ), 40.2 (s), 36.1 (s), 21.1 (s), 14.1 (s). HRMS (ESI, m/z): [M + H] calculated for $\mathrm{C}_{25} \mathrm{H}_{25} \mathrm{FN}_{3} \mathrm{O}_{3} \mathrm{~S}: 466.1595$; found: 466.1599. $[\alpha]_{D}^{23}=-154.1$ (c = 3.1, $\mathrm{CHCl}_{3}, 99 \%$ ee, $\left.>20: 1 \mathrm{dr}\right)$; HPLC (IB3, IPA $/ \mathrm{n}$-hexane $=10 / 90$, flow rate $=1 \mathrm{~mL} / \mathrm{min}, \mathrm{I}$ $=254 \mathrm{~nm}$ ) $t_{\mathrm{R}}=11.07 \mathrm{~min}$ (major), $9.07 \mathrm{~min}$ (minor).

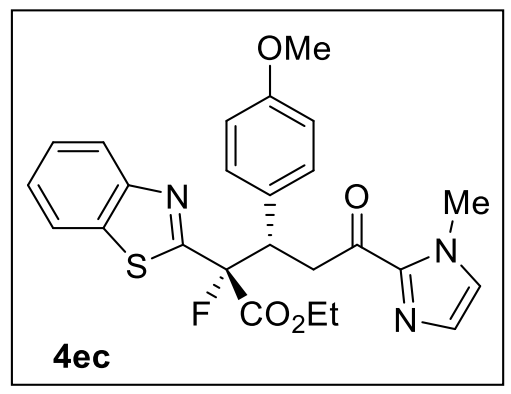

Ethyl

(2R,3S)-2-(benzo[d]thiazol-2-yl)-2-fluoro-3-(4-methoxyphenyl)-5-(1-methyl-1H-imidazol-2-yl)-5-

oxopentanoate $(\mathbf{4 e c})$ : The compound was prepared following the synthetic procedure $B$ from benzothiazolyl fluoroacetate $1 \mathrm{e}$ as limiting starting material $(48 \mathrm{mg}, 0.20 \mathrm{mmol})$. The desired product was isolated by silica gel column chromatography $\left(20: 80=\mathrm{EtOAc} /\right.$ petroleum ether, v/v) to afford a light yellow gel $(90 \mathrm{mg}, 93 \%$ yield $) ;{ }^{1} \mathbf{H ~ N M R}(500 \mathrm{MHz}$, $\mathrm{CDCl}_{3}$ ): $\delta 8.04(\mathrm{~d}, J=8.1 \mathrm{~Hz}, 1 \mathrm{H}), 7.74(\mathrm{~d}, J=8.0 \mathrm{~Hz}, 1 \mathrm{H}), 7.43(\mathrm{t}, J=7.5 \mathrm{~Hz}, 1 \mathrm{H}), 7.32(\mathrm{t}, J=7.5 \mathrm{~Hz}, 1 \mathrm{H}), 7.20$ (t, $J=$ $14.2 \mathrm{~Hz}, 3 \mathrm{H}), 6.98(\mathrm{~s}, 1 \mathrm{H}), 6.59$ (d, J=8.0 Hz, 2H), 4.77 (dd, J=33.6, $7.9 \mathrm{~Hz}, 1 \mathrm{H}$ ), 4.30 (d, J=27.8 Hz, 3H), 3.84 (s, 3H), $3.62(\mathrm{~s}, 3 \mathrm{H}), 3.47$ (s, $1 \mathrm{H}), 1.29$ (s, 3H). ${ }^{19} \mathrm{~F} \mathrm{NMR}\left(376 \mathrm{MHz}, \mathrm{CDCl}_{3}\right) \delta-162.78$ (d, J=27.8 Hz). ${ }^{13} \mathrm{C} \mathrm{NMR}\left(125 \mathrm{MHz}, \mathrm{CDCl}_{3}\right)$ : $\delta 188.7$ (s), 167.1 (s), 166.8 (d, J = 6.6 Hz), 158.6 (s), 152.9 (s), 142.5 (s), 135.1 (s), 131.0 (s), 128.7 (d, $J=16.4 \mathrm{~Hz}$ ), 127.0 (s), 126.1 (s), 125.4 (s), 123.8 (s), 121.6 (s), 113.5 (s), 99.5 (d, J = 194.8 Hz), 63.2 (s), 55.0 (s), 46.6 (d, J = 19.2 $\mathrm{Hz}$ ), 40.3 (s), 36.2 (s), 14.1 (s). HRMS (ESI, m/z): [M + H]+ calculated for C25H25FN3O4S: 482.1544; found: 482.1553. $[\alpha]_{D}^{23}=-163.5\left(\mathrm{c}=2.6, \mathrm{CHCl}_{3}, 97 \%\right.$ ee, $\left.>20: 1 \mathrm{dr}\right) ; \mathrm{HPLC}(\mathrm{IA}, \mathrm{IPA} / \mathrm{n}$-hexane $=25 / 75$, flow rate $=1 \mathrm{~mL} / \mathrm{min}, \mathrm{I}=254 \mathrm{~nm})$ $t_{R}=37.16 \min$ (major), 18.77 min (minor).

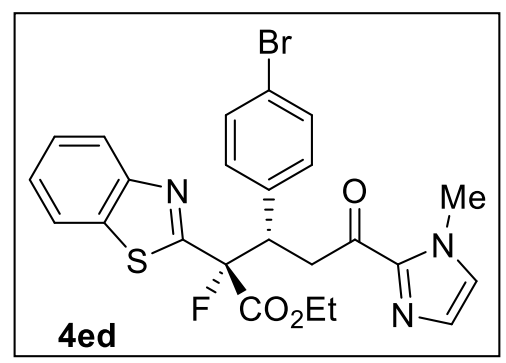

Ethyl (2R,3S)-2-(benzo[d]thiazol-2-yl)-3-(4-bromophenyl)-2-fluoro-5-(1-methyl-1H-imidazol-2-yl)-5-oxopentanoate (4ed): The compound was prepared following the synthetic procedure B from benzothiazolyl fluoroacetate 1e as limiting starting material $(48 \mathrm{mg}, 0.20 \mathrm{mmol})$. The desired product was isolated by silica gel column chromatography $(20: 80=$ EtOAc/petroleum ether, v/v) to afford a light yellow gel (93 mg, 88\% yield); ${ }^{1} \mathrm{H}$ NMR $\left(500 \mathrm{MHz}, \mathrm{CDCl}_{3}\right): \delta 8.03(\mathrm{~d}, J=8.2$ $\mathrm{Hz}, 1 \mathrm{H}), 7.73(\mathrm{~d}, J=8.0 \mathrm{~Hz}, 1 \mathrm{H}), 7.47-7.39(\mathrm{~m}, 1 \mathrm{H}), 7.36-7.29(\mathrm{~m}, 1 \mathrm{H}), 7.21-7.12(\mathrm{~m}, 4 \mathrm{H}), 7.10(\mathrm{~s}, 1 \mathrm{H}), 6.95(\mathrm{~s}, 1 \mathrm{H})$, 4.82 (ddd, $J=33.8,10.9,3.1 \mathrm{~Hz}, 1 \mathrm{H}$ ), 4.30 (q, $J=7.2 \mathrm{~Hz}, 2 \mathrm{H}$ ), 4.23 (dd, $J=17.5,11.0 \mathrm{~Hz}, 1 \mathrm{H}$ ), 3.81 (s, 3H), 3.43 (dd, $J$ $=17.6,3.2 \mathrm{~Hz}, 1 \mathrm{H}), 1.27$ (t, $J=7.1 \mathrm{~Hz}, 3 \mathrm{H}) .{ }^{19} \mathrm{~F} \mathrm{NMR}\left(376 \mathrm{MHz}, \mathrm{CDCl}_{3}\right) \delta-163.45$ (d, J=33.4 Hz). ${ }^{13} \mathrm{C} \mathrm{NMR}(125 \mathrm{MHz}$, $\mathrm{CDCl}_{3}$ ): $\delta 188.4$ (s), $166.6(\mathrm{~d}, J=25.2 \mathrm{~Hz}), 166.4(\mathrm{~d}, J=32.3 \mathrm{~Hz}), 152.9$ (s), 142.6 (s), 136.0 (s), 134.9 (s), 131.7 (s), 
131.2 (s), 129.2 (s), 127.2 (s), 126.3 (s), 125.6 (s), 123.8 (s), 121.7 (s), 121.5 (s), 99.2 (d, J = 195.5 Hz), 63.3 (s), 46.7 (d, $J=18.9 \mathrm{~Hz}$ ), 39.8 (s), 36.1 (s), 14.1 (s). HRMS (ESI, m/z): [M + H] $]^{+}$calculated for $\mathrm{C}_{24} \mathrm{H}_{22} \mathrm{BrFN}_{3} \mathrm{O}_{3} \mathrm{~S}$ : 530.0544 ; found: 530.0549. $[\alpha]_{D}^{23}=-168.1$ (c = 2.8, $\mathrm{CHCl}_{3}, 99 \%$ ee, $\left.>20: 1 \mathrm{dr}\right)$; HPLC (IB3, IPA $/ \mathrm{n}$-hexane $=10 / 90$, flow rate $=1 \mathrm{~mL} / \mathrm{min}, \mathrm{I}$ $=254 \mathrm{~nm}$ ) $\mathrm{t}_{\mathrm{R}}=11.90 \mathrm{~min}$ (major), $16.53 \mathrm{~min}$ (minor).

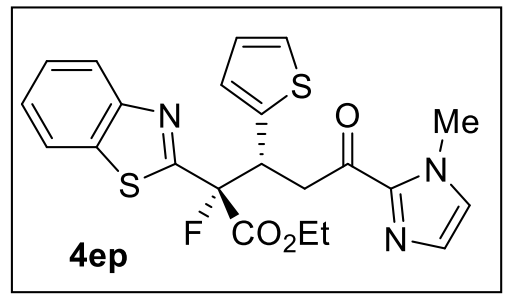

Ethyl (2R,3R)-2-(benzo[d]thiazol-2-yl)-2-fluoro-5-(1-methyl-1H-imidazol-2-yl)-5-oxo-3-(thiophen-2-yl)pentanoate (4ep): The compound was prepared following the synthetic procedure B from benzothiazolyl fluoroacetate 1e as limiting starting material $(48 \mathrm{mg}, 0.20 \mathrm{mmol})$. The desired product was isolated by silica gel column chromatography $(20: 80=$ EtOAc/petroleum ether, v/v) to afford a light yellow gel (80 mg, 87\% yield); $\mathbf{m p ~} 109-112{ }^{\circ} \mathrm{C} ;{ }^{\mathbf{1}} \mathbf{H ~} \mathbf{~ N M R}\left(500 \mathrm{MHz}, \mathrm{CDCl}_{3}\right)$ : $\delta 8.06(\mathrm{~d}, J=8.2 \mathrm{~Hz}, 1 \mathrm{H}), 7.77(\mathrm{~d}, J=8.0 \mathrm{~Hz}, 1 \mathrm{H}), 7.45(\mathrm{t}, J=7.6 \mathrm{~Hz}, 1 \mathrm{H}), 7.34(\mathrm{t}, J=7.6 \mathrm{~Hz}, 1 \mathrm{H}), 7.14(\mathrm{~s}, 1 \mathrm{H}), 7.03-$ $6.94(\mathrm{~m}, 2 \mathrm{H}), 6.91(\mathrm{~d}, J=2.6 \mathrm{~Hz}, 1 \mathrm{H}), 6.72-6.63(\mathrm{~m}, 1 \mathrm{H}), 5.15(\mathrm{dd}, J=32.2,10.1 \mathrm{~Hz}, 1 \mathrm{H}), 4.39-4.17(\mathrm{~m}, 3 \mathrm{H}), 3.85(\mathrm{~s}$, $3 \mathrm{H}), 3.48(\mathrm{~d}, J=16.1 \mathrm{~Hz}, 1 \mathrm{H}), 1.28(\mathrm{t}, J=5.7 \mathrm{~Hz}, 3 \mathrm{H}) .{ }^{19} \mathrm{~F}$ NMR $\left(376 \mathrm{MHz}, \mathrm{CDCl}_{3}\right) \delta-161.76(\mathrm{~d}, J=32.8 \mathrm{~Hz}) .{ }^{13} \mathrm{C} \mathrm{NMR}$ $\left(125 \mathrm{MHz}, \mathrm{CDCl}_{3}\right): \delta 188.1$ (s), 166.6 (d, J=5.5 Hz), 166.4 (s), 152.9 (s), 142.6 (s), 138.9 (s), 135.2 (s), 129.1 (s), 128.4 (s), 127.8 (s), 127.2 (s), 126.4 (d, J = $22.7 \mathrm{~Hz}$ ), 125.6 (s), 125.2 (s), 123.9 (s), 121.7 (s), 98.7 (d, J = 195.4 Hz), 63.3 (s), 43.0 (d, $J=20.0 \mathrm{~Hz}$ ), 41.3 (s), 36.2 (s), 14.1 (s). HRMS (ESI, m/z): [M + H] calculated for $\mathrm{C}_{22} \mathrm{H}_{21} \mathrm{FN}_{3} \mathrm{O}_{3} \mathrm{~S}_{2}: 458.1003$; found: 458.1009. [ []$_{D}{ }_{D}^{23}=-142.4\left(\mathrm{C}=0.80, \mathrm{CHCl}_{3}, 96 \%\right.$ ee, $>20: 1 \mathrm{dr}$ ); HPLC (IB3, IPA/n-hexane = 10/90, flow rate $=1$ $\mathrm{mL} / \mathrm{min}, \mathrm{I}=254 \mathrm{~nm}$ ) $\mathrm{t}_{\mathrm{R}}=11.52 \mathrm{~min}$ (major), $15.80 \mathrm{~min}$ (minor).

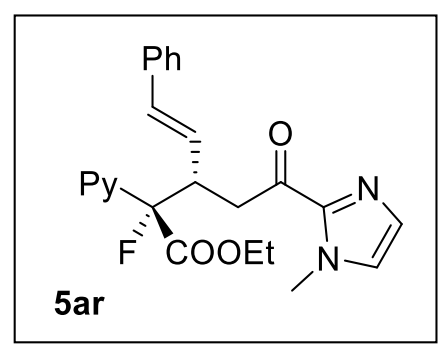

Ethyl (2S,3S,E)-2-fluoro-3-(2-(1-methyl-1H-imidazol-2-yl)-2-oxoethyl)-5-phenyl-2-(pyridin-2-yl)pent-4-enoate (5ar): The compound was prepared following the synthetic procedure A from pyridinyl fluoroacetate 1a as limiting starting material $(36 \mathrm{mg}, 0.20 \mathrm{mmol})$. The desired product was isolated by silica gel column chromatography $(30: 70=$ EtOAc/petroleum ether, v/v) to afford a light yellow gel (51 mg, $60 \%$ yield); ${ }^{1} \mathrm{H}$ NMR $\left(500 \mathrm{MHz}, \mathrm{CDCl}_{3}\right): \delta 8.61$ (d, $J=4.5$ $\mathrm{Hz}, 1 \mathrm{H}), 7.68-7.61(\mathrm{~m}, 1 \mathrm{H}), 7.55(\mathrm{~d}, J=8.0 \mathrm{~Hz}, 1 \mathrm{H}), 7.23-7.04(\mathrm{~m}, 7 \mathrm{H}), 6.98(\mathrm{~s}, 1 \mathrm{H}), 6.29(\mathrm{~d}, J=15.9 \mathrm{~Hz}, 1 \mathrm{H}), 5.93$ (dd, $J=15.9,9.3 \mathrm{~Hz}, 1 \mathrm{H}), 4.42-4.31(\mathrm{~m}, 1 \mathrm{H}), 4.27-4.21(\mathrm{~m}, 2 \mathrm{H}), 3.94-3.84(\mathrm{~m}, 4 \mathrm{H}), 3.24(\mathrm{dd}, J=16.7,2.8 \mathrm{~Hz}, 1 \mathrm{H})$, 1.24 (t, $J=7.1 \mathrm{~Hz}, 3 \mathrm{H}) .{ }^{19} \mathrm{~F}$ NMR $\left(376 \mathrm{MHz}, \mathrm{CDCl}_{3}\right) \delta-174.29$ (d, $\left.J=33.5 \mathrm{~Hz}\right) .{ }^{13} \mathrm{C} \mathrm{NMR}\left(125 \mathrm{MHz}, \mathrm{CDCl}_{3}\right): \delta 189.7(\mathrm{~s})$, 168.5 (d, J = 25.3 Hz), 156.3 (d, J = 27.2 Hz), 149.1 (s), 143.0 (s), 137.1 (s), 136.8 (s), 134.4 (s), 131.0 (s), 128.3 (s), 127.3 (s), 127.0 (s), 126.3 (s), 125.8 (s), 123.3 (s), 120.4 (d, J = 9.3 Hz), 99.7 (d, J = 193.8 Hz), 62.3 (s), 44.5 (d, J = 19.4

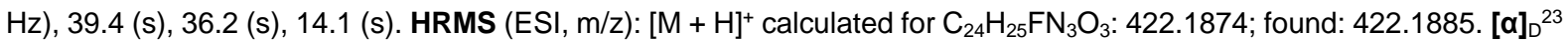


$=-51\left(\mathrm{c}=0.50, \mathrm{CHCl}_{3}, 99 \%\right.$ ee, $\left.>20: 1 \mathrm{dr}\right) ; \mathrm{HPLC}(\mathrm{IA3}, \mathrm{IPA} / \mathrm{n}$-hexane $=30 / 70$, flow rate $=0.8 \mathrm{~mL} / \mathrm{min}, \mathrm{I}=254 \mathrm{~nm}) \mathrm{t}_{\mathrm{R}}=$ $35.22 \mathrm{~min}$ (major), $12.08 \mathrm{~min}$ (minor).

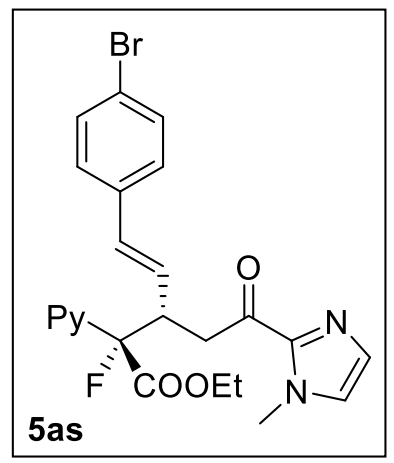

Ethyl (2S,3S,E)-5-(4-bromophenyl)-2-fluoro-3-(2-(1-methyl-1H-imidazol-2-yl)-2-oxoethyl)-2-(pyridin-2-yl)pent-4enoate (5as): The compound was prepared following the synthetic procedure A from pyridinyl fluoroacetate 1a as limiting starting material $(36 \mathrm{mg}, 0.20 \mathrm{mmol})$. The desired product was isolated by silica gel column chromatography $(30: 70=$ EtOAc/petroleum ether, v/v) to afford a light yellow gel (56 mg, $56 \%$ yield); ${ }^{1} \mathrm{H}$ NMR $\left(500 \mathrm{MHz}, \mathrm{CDCl}_{3}\right): \delta 8.60$ (d, $J=4.3$ $\mathrm{Hz}, 1 \mathrm{H}), 7.65$ (td, $J=7.8,1.7 \mathrm{~Hz}, 1 \mathrm{H}), 7.53(\mathrm{~d}, J=7.2 \mathrm{~Hz}, 1 \mathrm{H}), 7.26(\mathrm{~d}, J=8.5 \mathrm{~Hz}, 2 \mathrm{H}), 7.19-7.12(\mathrm{~m}, 2 \mathrm{H}), 6.95(\mathrm{dd}, J$ $=18.3,10.0 \mathrm{~Hz}, 3 \mathrm{H}), 6.23(\mathrm{~d}, J=15.9 \mathrm{~Hz}, 1 \mathrm{H}), 5.92(\mathrm{dd}, J=15.9,9.3 \mathrm{~Hz}, 1 \mathrm{H}), 4.42-4.28(\mathrm{~m}, 1 \mathrm{H}), 4.27-4.22(\mathrm{~m}, 2 \mathrm{H})$, $3.93-3.85(\mathrm{~m}, 4 \mathrm{H}), 3.23(\mathrm{dd}, J=16.8,2.9 \mathrm{~Hz}, 1 \mathrm{H}), 1.24-1.21(\mathrm{~m}, 5 \mathrm{H}) .{ }^{19} \mathrm{~F}$ NMR $\left(376 \mathrm{MHz}, \mathrm{CDCl}_{3}\right) \delta-174.19(\mathrm{~d}, J=$ $30.0 \mathrm{~Hz}$ ). ${ }^{13} \mathrm{C}$ NMR $\left(125 \mathrm{MHz}, \mathrm{CDCl}_{3}\right): \delta 189.6$ (s), 168.3 (d, $\left.J=25.5 \mathrm{~Hz}\right), 156.2$ (d, $\left.J=27.4 \mathrm{~Hz}\right), 149.1$ (s), 143.0 (s), 136.8 (s), 136.0 (s), 133.2 (s), 131.4 (s), 130.9 (s), 128.9 (s), 127.8 (s), 127.0 (s), 126.7 (s), 123.3 (s), 121.1 (s), 120.3 (d, $J=9.1 \mathrm{~Hz}$ ), 99.5 (d, $J=194.2 \mathrm{~Hz}$ ), 62.4 (s), 44.4 (d, $J=19.4 \mathrm{~Hz}$ ), 39.3 (s), 36.2 (s), 14.1 (s). HRMS (ESI, m/z): [M + H] calculated for $\mathrm{C}_{24} \mathrm{H}_{24} \mathrm{BrFN}_{3} \mathrm{O}_{3}$ : 500.0980; found: 500.0986. [a] ${ }_{D}^{23}=-61.4$ (c = 0.50, $\mathrm{CHCl}_{3}, 96 \%$ ee, 10:1 dr); HPLC (IA3, $\mathrm{IPA} / \mathrm{n}$-hexane $=30 / 70$, flow rate $=0.8 \mathrm{~mL} / \mathrm{min}, \mathrm{I}=254 \mathrm{~nm}$ ) $\mathrm{t}_{\mathrm{R}}=53.07 \mathrm{~min}$ (major), $15.26 \mathrm{~min}$ (minor).

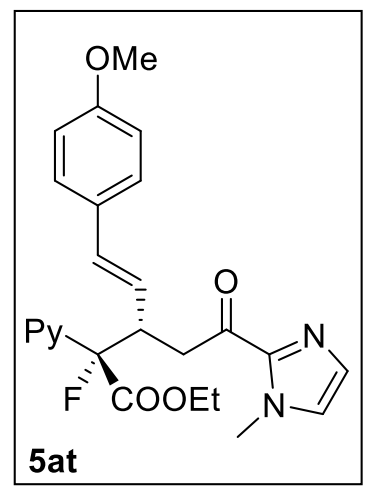

Ethyl (2S,3S,E)-2-fluoro-5-(4-methoxyphenyl)-3-(2-(1-methyl-1H-imidazol-2-yl)-2-oxoethyl)-2-(pyridin-2-yl)pent-4enoate (5at): The compound was prepared following the synthetic procedure A from pyridinyl fluoroacetate 1a as limiting starting material $(36 \mathrm{mg}, 0.20 \mathrm{mmol})$. The desired product was isolated by silica gel column chromatography $(30: 70=$ EtOAc/petroleum ether, v/v) to afford a light yellow gel (59 mg, 65\% yield); ${ }^{1} \mathrm{H}$ NMR $\left(500 \mathrm{MHz}, \mathrm{CDCl}_{3}\right): \delta 8.59(\mathrm{~d}, \mathrm{~J}=4.4$ $\mathrm{Hz}, 1 \mathrm{H}), 7.72-7.59(\mathrm{~m}, 1 \mathrm{H}), 7.53(\mathrm{~d}, J=7.6 \mathrm{~Hz}, 1 \mathrm{H}), 7.19-7.10(\mathrm{~m}, 2 \mathrm{H}), 7.03-6.85(\mathrm{~m}, 3 \mathrm{H}), 6.68(\mathrm{~d}, J=8.7 \mathrm{~Hz}, 2 \mathrm{H})$, $6.21(\mathrm{~d}, J=15.8 \mathrm{~Hz}, 1 \mathrm{H}), 5.77(\mathrm{dd}, J=15.9,9.3 \mathrm{~Hz}, 1 \mathrm{H}), 4.40-4.27(\mathrm{~m}, 1 \mathrm{H}), 4.26-4.21(\mathrm{~m}, 2 \mathrm{H}), 3.90-3.81(\mathrm{~m}, 4 \mathrm{H})$, $3.70(\mathrm{~s}, 3 \mathrm{H}), 3.19$ (dd, $J=16.6,2.9 \mathrm{~Hz}, 1 \mathrm{H}), 1.23(\mathrm{t}, J=7.1 \mathrm{~Hz}, 3 \mathrm{H}) .{ }^{19} \mathrm{~F}$ NMR $\left(376 \mathrm{MHz}, \mathrm{CDCl}_{3}\right) \delta-174.32(\mathrm{~d}, J=32.7$ $\mathrm{Hz}) .{ }^{13} \mathrm{C}$ NMR (125 MHz, CDCl$): \delta 189.9$ (s), 168.5 (d, $\left.J=25.2 \mathrm{~Hz}\right), 159.0$ (s), 156.4 (d, J = 27.0 Hz), 149.1 (s), 143.2 (s), 136.7 (s), 133.7 (s), 130.8 (s), 129.9 (s), 129.0 (s), 127.4 (s), 127.0 (s), 123.5 (s), 123.2 (s), 120.4 (d, J = 9.1 Hz), 113.7 (s), 99.7 (d, $J=193.9 \mathrm{~Hz}$ ), 62.3 (s), 55.3 (s), 44.5 (d, $J=19.7 \mathrm{~Hz}$ ), 39.4 (s), 36.1 (s), 14.1 (s). HRMS (ESI, m/z): [M 
$+\mathrm{H}]^{+}$calculated for $\mathrm{C}_{25} \mathrm{H}_{27} \mathrm{FN}_{3} \mathrm{O}_{4}: 452.1980$; found: 452.1983. [ $\left.\alpha\right]_{D}^{23}=-63.75$ (c=1.2, $\mathrm{CHCl}_{3}, 97 \%$ ee, 10:1 dr); HPLC $\left(\mathrm{IA3}, \mathrm{IPA} / \mathrm{n}\right.$-hexane $=30 / 70$, flow rate $=0.8 \mathrm{~mL} / \mathrm{min}, \mathrm{I}=254 \mathrm{~nm}$ ) $\mathrm{t}_{\mathrm{R}}=47.72 \mathrm{~min}$ (major), $15.35 \mathrm{~min}$ (minor).

\section{General procedure and characterization of 7:}

To a solution of compound 3ad $(0.2 \mathrm{mmol})$ in dry $\mathrm{CH}_{3} \mathrm{CN}(1.5 \mathrm{ml})$ at $35^{\circ} \mathrm{C}$, was added $4 \AA$ molecular sieve $(150 \mathrm{mg})$ and MeOTf $(0.3 \mathrm{mmol})$ under $\mathrm{N}_{2}$ atmosphere. After stirring for 5 hours at $35^{\circ} \mathrm{C}, \mathrm{MeOH}(500 \mu \mathrm{L})$ and $\mathrm{DBU}(0.3 \mathrm{mmol})$ was added. The resulting mixture was allowed to stir for $30 \mathrm{~min}$ at $35^{\circ} \mathrm{C}$ and the reaction mixture was diluted with EtOAc (1 $\mathrm{mL}$ ) and washed with saturated $\mathrm{NaHCO}_{3}$ and brine. The organic layer was dried over $\mathrm{MgSO}_{4}$. Then, the drying agent was removed by filtration, and the resulting solution was evaporated under reduced pressure and the residue was purified over silica gel by column chromatography to afford the products.

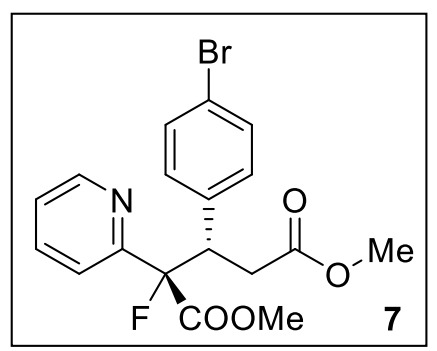

Dimethyl (2S,3S)-3-(4-bromophenyl)-2-fluoro-2-(pyridin-2-yl)pentanedioate (7): The desired product was isolated by silica gel column chromatography (30:70 = EtOAc/petroleum ether, v/v) to afford a light yellow gel $(70 \mathrm{mg}, 85 \%$ yield $) ;{ }^{1} \mathbf{H}$ NMR $\left(500 \mathrm{MHz}, \mathrm{CDCl}_{3}\right) \delta 8.54(\mathrm{~d}, J=4.3 \mathrm{~Hz}, 1 \mathrm{H}), 7.56-7.43(\mathrm{~m}, 1 \mathrm{H}), 7.22-7.13(\mathrm{~m}, 3 \mathrm{H}), 7.13-7.05(\mathrm{~m}, 1 \mathrm{H}), 7.03-$ $6.89(\mathrm{~m}, 2 \mathrm{H}), 4.56$ (ddd, $J=33.5,11.3,3.7 \mathrm{~Hz}, 1 \mathrm{H}), 3.82(\mathrm{~s}, 3 \mathrm{H}), 3.51(\mathrm{~s}, 3 \mathrm{H}), 3.01$ (dd, $J=15.9,11.4 \mathrm{~Hz}, 1 \mathrm{H}), 2.88(\mathrm{dd}$, $J=15.9,3.7 \mathrm{~Hz}, 1 \mathrm{H}) .{ }^{19} \mathrm{~F}$ NMR $\left(376 \mathrm{MHz}, \mathrm{CDCl}_{3}\right) \delta-176.53(\mathrm{~d}, J=34.8 \mathrm{~Hz}) .{ }^{13} \mathrm{C}$ NMR $\left(126 \mathrm{MHz}, \mathrm{CDCl}_{3}\right) \delta 171.2(\mathrm{~s})$, 168.6 (d, $J=25.5 \mathrm{~Hz}$ ), 155.4 (d, J = 27.9 Hz), 148.9 (s), 137.0 (s), 136.1 (s), 131.3 (s), 131.1 (s), 123.4 (s), 121.3 (s), 120.1 (d, $J=9.7 \mathrm{~Hz}$ ), 99.4 (d, $J=195.3 \mathrm{~Hz}$ ), 53.5 (s), 51.9 (s), 46.9 (d, $J=18.6 \mathrm{~Hz}$ ), 35.7 (d, $J=4.3 \mathrm{~Hz}$ ). HRMS (ESI,

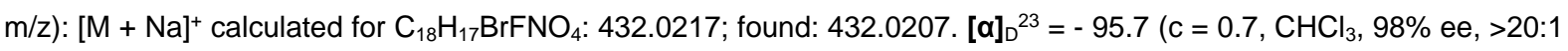
$\mathrm{dr})$; HPLC (IC3, IPA $/ \mathrm{n}$-hexane $=30 / 70$, flow rate $=1 \mathrm{~mL} / \mathrm{min}, \mathrm{I}=254 \mathrm{~nm}$ ) $\mathrm{t}_{\mathrm{R}}=10.32 \mathrm{~min}$ (major), $5.41 \mathrm{~min}$ (minor).

\section{General procedure and characterization of 8:}

To a solution of compound 3aa $(0.2 \mathrm{mmol})$ in dry $\mathrm{CH}_{3} \mathrm{CN}(1.5 \mathrm{ml})$ at $35^{\circ} \mathrm{C}$, was added $4 \AA$ molecular sieve $(150 \mathrm{mg})$. The suspension was stirred vigorously under a positive pressure of nitrogen for 2 hours then MeOTf (0.4 mmol) was added and the reaction was stirred for an additional 2 hours under $\mathrm{N}_{2}$ atmosphere. Then the reaction mixture was filtered through a short plug of oven dried Celite. The vial and Celite were washed with $1 \mathrm{ml} \mathrm{EtOAc}$ and the solvent was removed in vacuo. The resulting solid was dissolved in THF $(3 \mathrm{ml})$ and the vial cooled to $-78^{\circ} \mathrm{C}$ before phenylmagnesium bromide $(2.5 \mathrm{eq})$ was added very slowly. The reaction was then stirred for $30 \mathrm{~min}$ before quenched by saturated aqueous $\mathrm{NaHCO}_{3}(2 \mathrm{ml})$ and water $(10 \mathrm{ml})$ and the reaction allowed to warm to ambient temperature with stirring. The aqueous layer was then extracted with diethyl ether $(3 \times 15 \mathrm{ml})$ and the combined organic dried over anhydrous $\mathrm{Na}_{2} \mathrm{SO}_{4}$ and the solvent was removed in vacuo. The product was purified by flash chromatography $\left(\mathrm{PE}: \mathrm{Et}_{2} \mathrm{O}\right)$ to give the title compound 8 . 


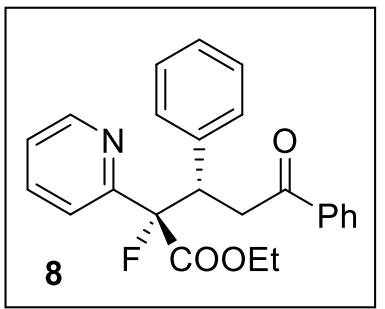

Ethyl (2S,3S)-2-fluoro-5-oxo-3,5-diphenyl-2-(pyridin-2-yl)pentanoate (8): The desired product was isolated by silica gel column chromatography $\left(10: 90=\mathrm{Et}_{2} \mathrm{O} /\right.$ petroleum ether, $\left.\mathrm{v} / \mathrm{v}\right)$ to afford a light yellow gel $(55 \mathrm{mg}, 70 \%$ yield); $\mathbf{m p} 123-$ $126{ }^{\circ} \mathrm{C} ;{ }^{1} \mathbf{H}$ NMR $\left(500 \mathrm{MHz}, \mathrm{CDCl}_{3}\right) \delta 8.54(\mathrm{~d}, J=4.3 \mathrm{~Hz}, 1 \mathrm{H}), 7.96-7.84(\mathrm{~m}, 2 \mathrm{H}), 7.56-7.37(\mathrm{~m}, 4 \mathrm{H}), 7.20-7.10(\mathrm{~m}$, $3 \mathrm{H}$ ), $7.09-6.95(\mathrm{~m}, 4 \mathrm{H}), 4.79$ (ddd, $J=34.1,10.7,3.1 \mathrm{~Hz}, 1 \mathrm{H}), 4.30(\mathrm{q}, J=7.2 \mathrm{~Hz}, 2 \mathrm{H}), 3.84(\mathrm{dd}, J=16.9,10.7 \mathrm{~Hz}, 1 \mathrm{H}$ ), 3.48 (dd, $J=16.9,3.1 \mathrm{~Hz}, 1 \mathrm{H}), 1.25$ (t, $J=7.1 \mathrm{~Hz}, 3 \mathrm{H}) .{ }^{19} \mathrm{~F} \mathrm{NMR}\left(376 \mathrm{MHz}, \mathrm{CDCl}_{3}\right) \delta-174.32(\mathrm{~d}, J=32.6 \mathrm{~Hz}) .{ }^{13} \mathrm{C} \mathrm{NMR}$ $\left(126 \mathrm{MHz}, \mathrm{CDCl}_{3}\right.$ ) $\delta 196.9$ (s), 168.6 (d, J = 25.1 Hz), 155.9 (d, J = 24.3 Hz), 148.5 (s), 137.6 (s), 136.8 (d, J = $\left.12.6 \mathrm{~Hz}\right)$, 133.1 (s), 129.7 (s), 128.6 (s), 128.2 (s), 127.9 (s), 127.0 (s), 123.1 (s), 120.4 (d, J = 7.7 Hz), 100.1 (d, J = 195.3 Hz), 62.6 (s), 46.9 (d, $J=19.0 \mathrm{~Hz}$ ), 40.0 (s), 14.1 (s). HRMS (ESI, m/z): [M + H] $]^{+}$calculated for $\mathrm{C}_{24} \mathrm{H}_{23} \mathrm{FNO}_{3}: 392.1656$; found: 392.1667. $[\alpha]_{D}^{23}=-96.4\left(\mathrm{c}=0.25, \mathrm{CHCl}_{3}, 99 \%\right.$ ee, $\left.>20: 1 \mathrm{dr}\right)$; HPLC (ID, IPA $/ \mathrm{n}$-hexane $=30 / 70$, flow rate $=1 \mathrm{~mL} / \mathrm{min}, \mathrm{I}=$ $254 \mathrm{~nm}) t_{\mathrm{R}}=16.94 \mathrm{~min}$ (major), $8.66 \mathrm{~min}$ (minor). 
Crystal structure of 3ad: (CCDC 2110157) Thermal ellipsoids are drawn at $50 \%$ probability in this ORTEP representation.
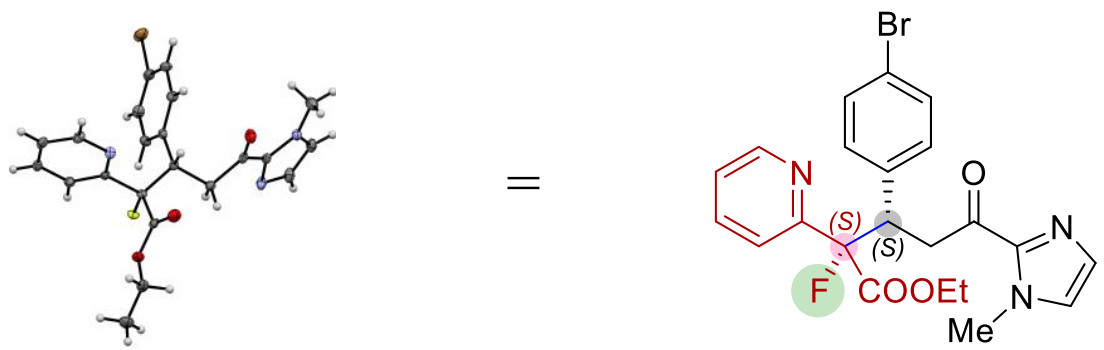

Crystal structure of 4ep: (CCDC 2110161) Thermal ellipsoids are drawn at $50 \%$ probability in this ORTEP representation.

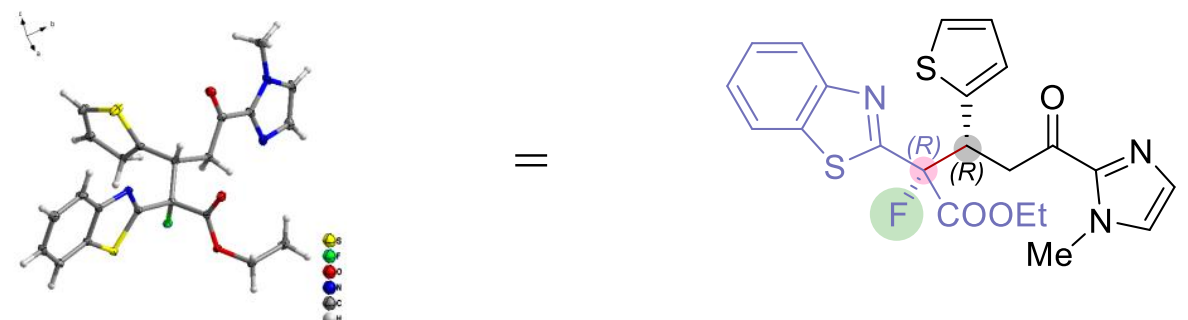

Table for the crystallographic data for 3ad and 4ep.

\begin{tabular}{|c|c|c|}
\hline Identification code & 4 ep & $3 a d$ \\
\hline Empirical formula & $\mathrm{C}_{44} \mathrm{H}_{41} \mathrm{~F}_{2} \mathrm{~N}_{6} \mathrm{O}_{6} \mathrm{~S}_{4}$ & $\mathrm{C}_{22} \mathrm{H}_{21} \mathrm{BrFN}_{3} \mathrm{O}_{3}$ \\
\hline Formula weight & 916.07 & 474.33 \\
\hline Temperature/K & 273.15 & 273.15 \\
\hline Crystal system & triclinic & orthorhombic \\
\hline Space group & $\mathrm{P} 1$ & $\mathrm{P} 22_{1} 2_{1} 2_{1}$ \\
\hline $\mathrm{a} / \AA ̊$ & $8.4924(9)$ & $10.6445(9)$ \\
\hline $\mathrm{b} / \AA$ & $10.5805(12)$ & $11.5113(10)$ \\
\hline $\mathrm{c} / \AA ̊$ & $12.4778(14)$ & $17.1194(14)$ \\
\hline$\alpha /^{\circ}$ & $93.506(3)$ & 90 \\
\hline$\beta /^{\circ}$ & $102.760(3)$ & 90 \\
\hline$Y /^{\circ}$ & $99.165(3)$ & 90 \\
\hline Volume $/ \AA^{3}$ & $1074.1(2)$ & $2097.7(3)$ \\
\hline Z & 1 & 4 \\
\hline$\rho_{\text {calc }} \mathrm{g} / \mathrm{cm}^{3}$ & 1.416 & 1.502 \\
\hline$\mu / \mathrm{mm}^{-1}$ & 0.286 & 1.997 \\
\hline$F(000)$ & 477.0 & 968.0 \\
\hline Crystal size $/ \mathrm{mm}^{3}$ & $0.45 \times 0.34 \times 0.25$ & $0.35 \times 0.34 \times 0.23$ \\
\hline Radiation & $\operatorname{MoKa}(\lambda=0.71073)$ & $\operatorname{MoKa}(\lambda=0.71073)$ \\
\hline
\end{tabular}




\begin{tabular}{|c|c|c|}
\hline $2 \Theta$ range for data collection $/{ }^{\circ}$ & 5.414 to 56.612 & 4.264 to 56.622 \\
\hline Index ranges & $-11 \leq h \leq 11,-14 \leq k \leq 14,-16 \leq \mathrm{I} \leq 16$ & $-14 \leq \mathrm{h} \leq 14,-15 \leq \mathrm{k} \leq 15,-22 \leq \mathrm{I} \leq 22$ \\
\hline Reflections collected & 21799 & 44805 \\
\hline Independent reflections & $10640\left[R_{\text {int }}=0.0397, R_{\text {sigma }}=0.0671\right]$ & $5214\left[R_{\text {int }}=0.0481, R_{\text {sigma }}=0.0319\right]$ \\
\hline Data/restraints/parameters & $10640 / 3 / 563$ & $5214 / 0 / 273$ \\
\hline Goodness-of-fit on $\mathrm{F}^{2}$ & 1.051 & 0.949 \\
\hline Final $R$ indexes $[\mathrm{I}>=2 \sigma(\mathrm{I})]$ & $R_{1}=0.0445, w R_{2}=0.0911$ & $\mathrm{R}_{1}=0.0228, w \mathrm{R}_{2}=0.0609$ \\
\hline Final $\mathrm{R}$ indexes [all data] & $R_{1}=0.0576, w R_{2}=0.1004$ & $\mathrm{R}_{1}=0.0254, \mathrm{wR}_{2}=0.0626$ \\
\hline Largest diff. peak/hole / e $\AA^{-3}$ & $0.35 /-0.55$ & $0.31 /-0.40$ \\
\hline Flack parameter & $0.02(3)$ & $0.014(3$ \\
\hline
\end{tabular}


Check CIF file data of 3ad (CCDC 2110157) Thermal ellipsoids are drawn at 50\% probability in this ORTEP representation:

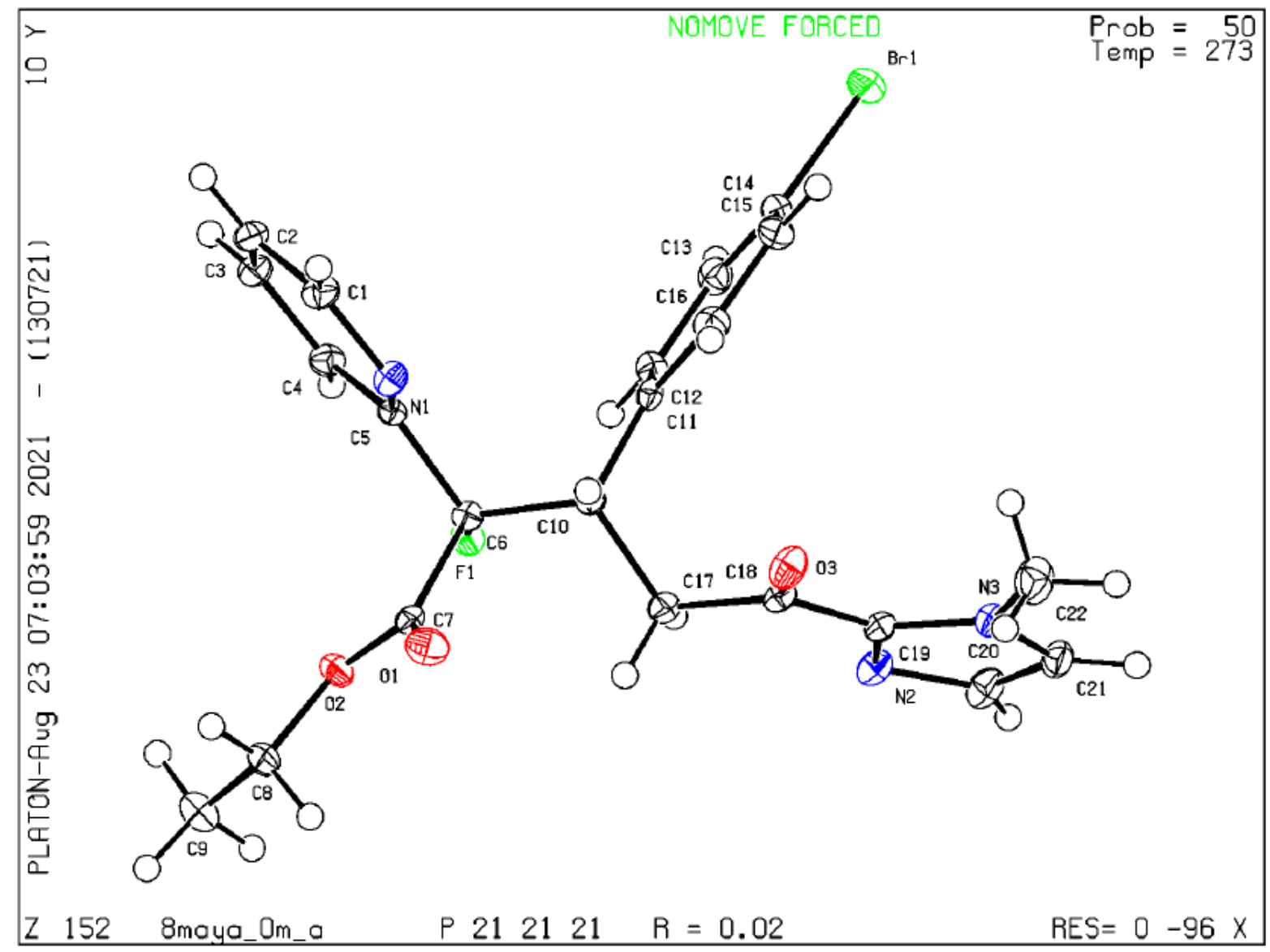


Check CIF file data of 4ep (CCDC 2110161) Thermal ellipsoids are drawn at $50 \%$ probability in this ORTEP representation:

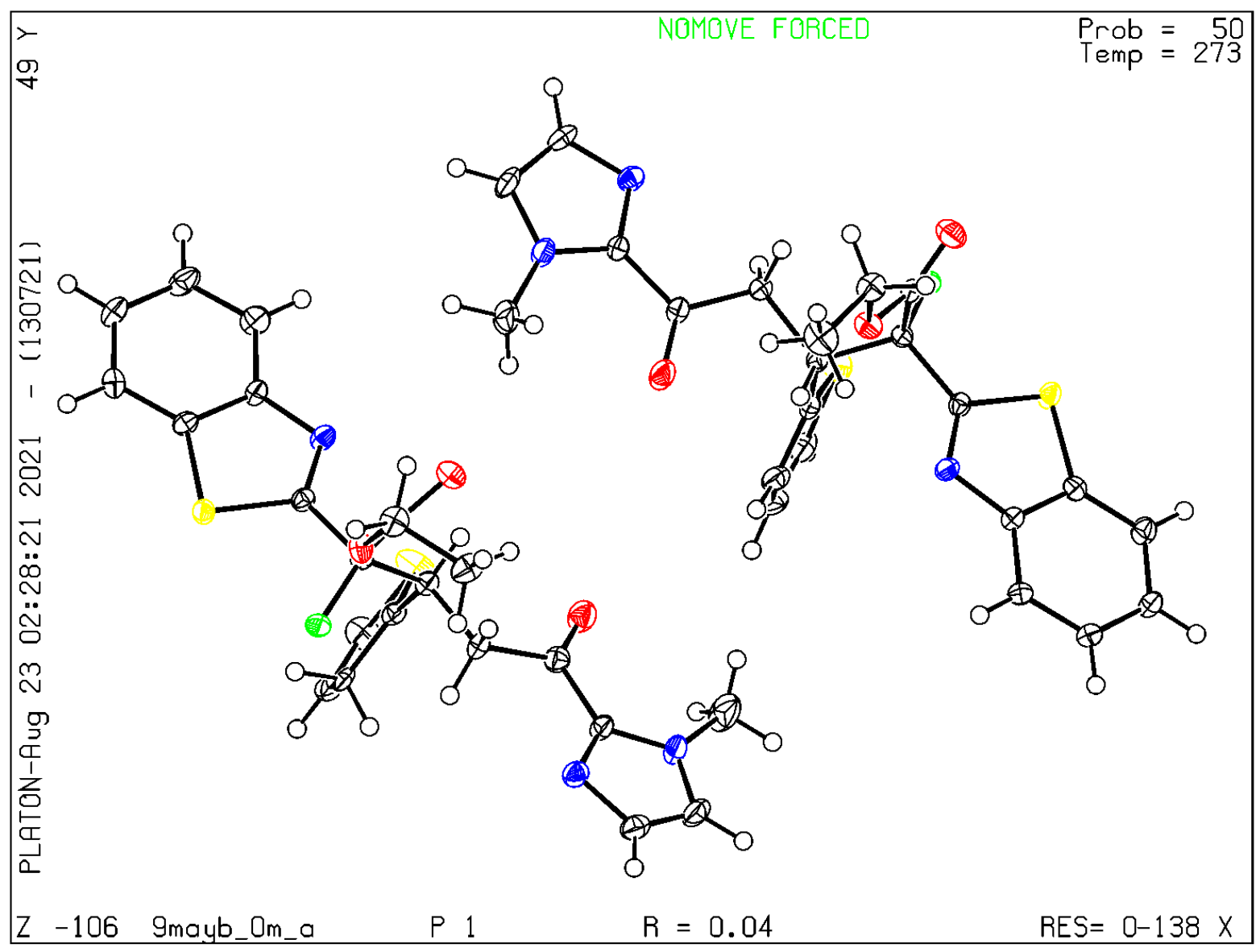




\section{Proposed transition state and stereochemical outcome:}

Absolute stereochemistry of the products 4ep (CCDC 2110161) and 3ad (CCDC 2110157) was determined by crystal structures. This model shows the mode of binding and the origin of the stereoselectivity. Where the Si face of the electrophile is reacting with the Re or Si face of the nucleophile. As mentioned earlier, due to different priority order of the nucleophiles the stereochemical outcome is different.
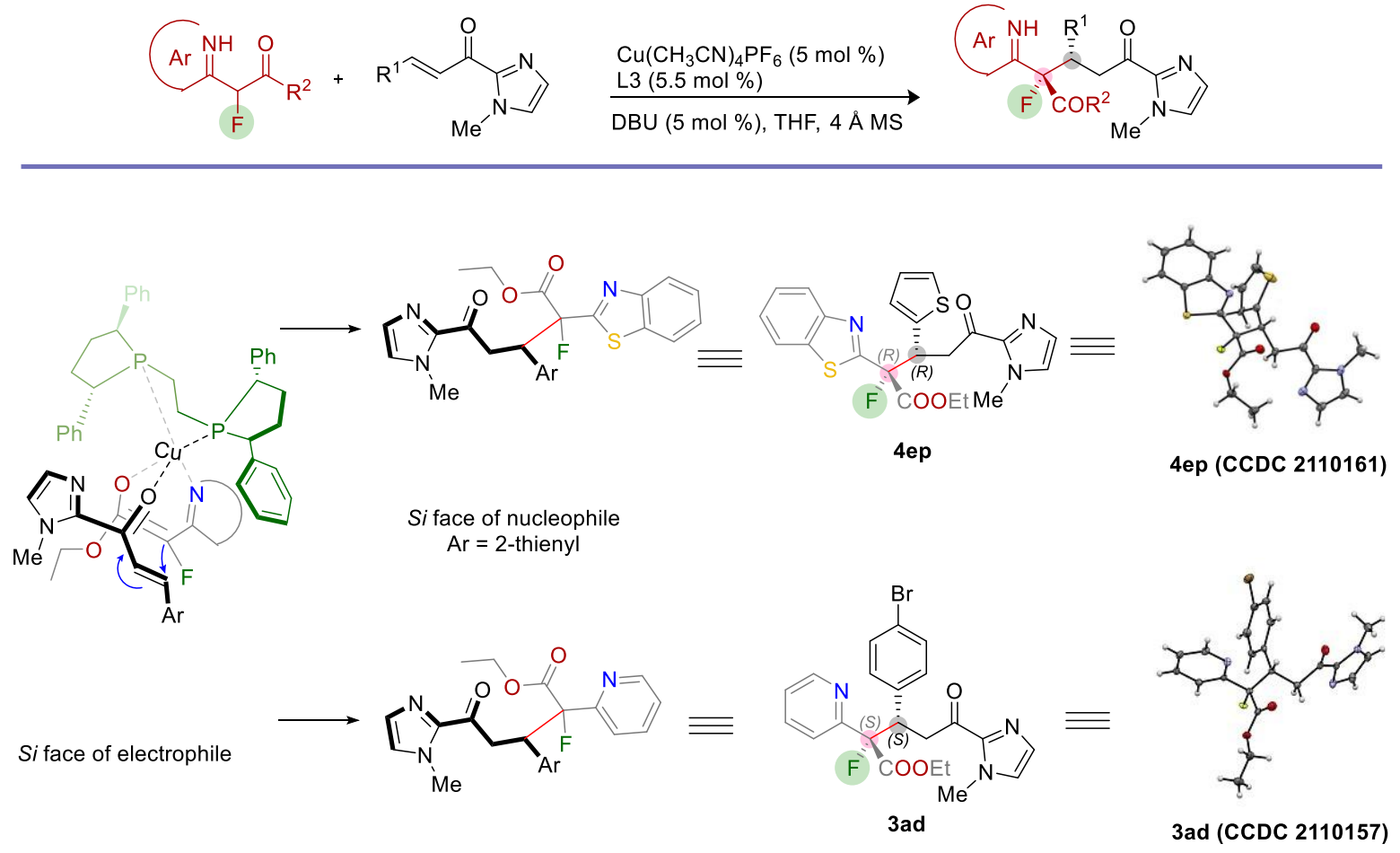

Re face of nucleophile $\mathrm{Ar}=4-\mathrm{Br}-\mathrm{C}_{6} \mathrm{H}_{4}$ 
Spectra of products 3,4 and 5: 


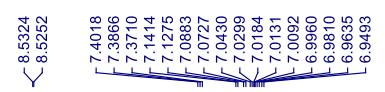

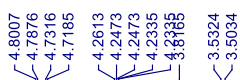

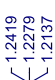

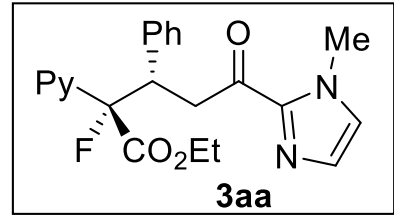

3aa

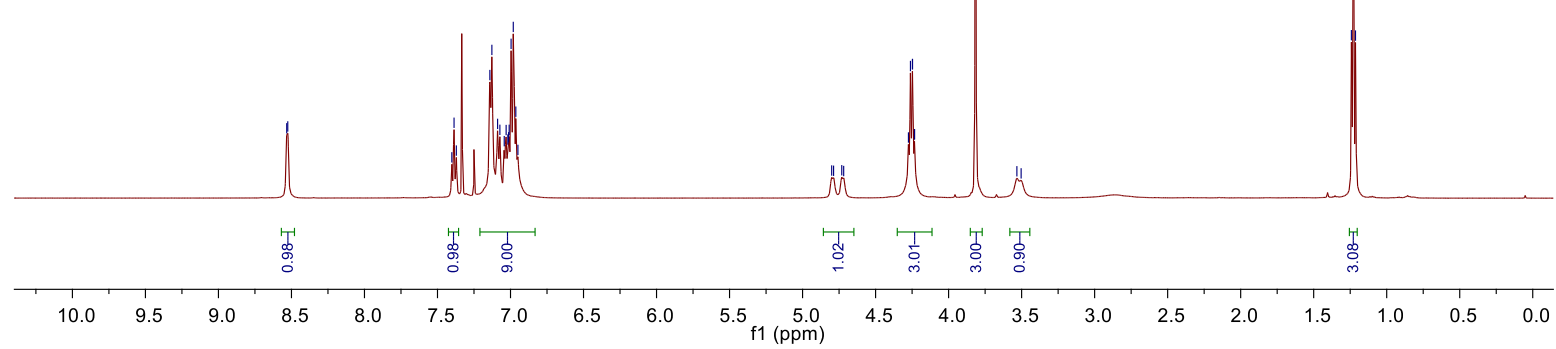

$500 \mathrm{MHz}{ }^{1} \mathrm{H}$ NMR spectra of compound 3aa in $\mathrm{CDCl}_{3}$
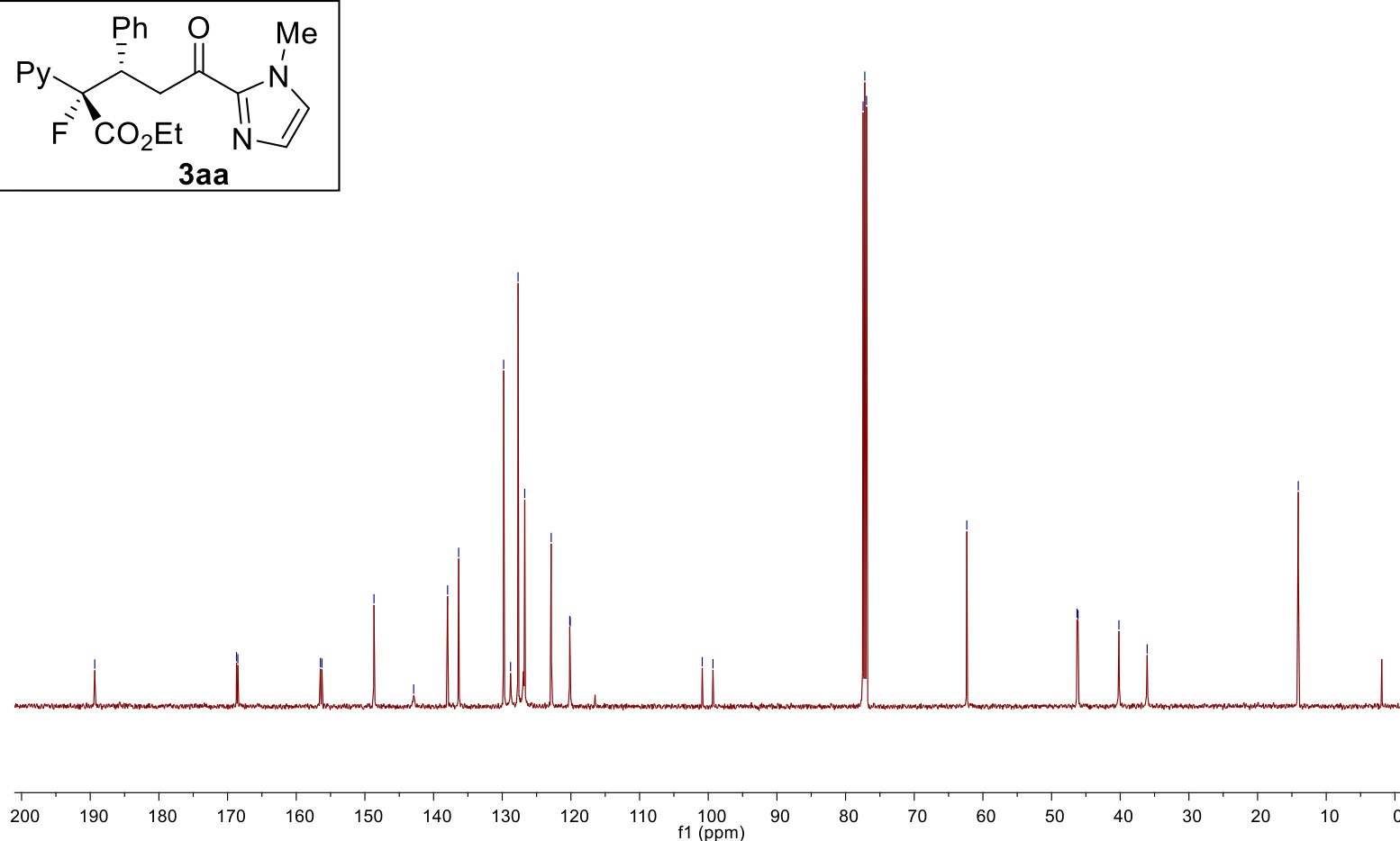

$125 \mathrm{MHz}{ }^{13} \mathrm{C}$ NMR spectra of compound 3aa in $\mathrm{CDCl}_{3}$ 

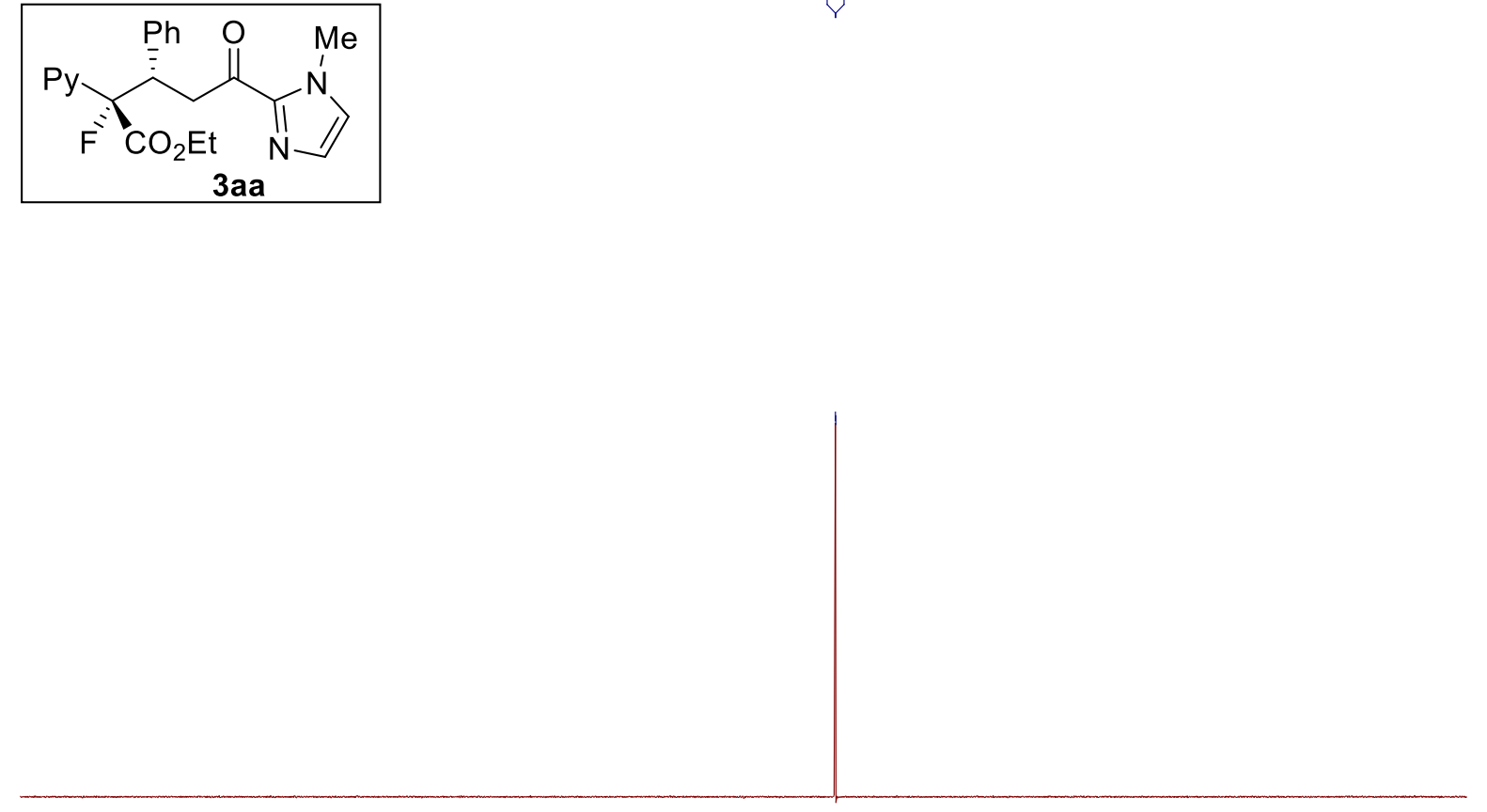

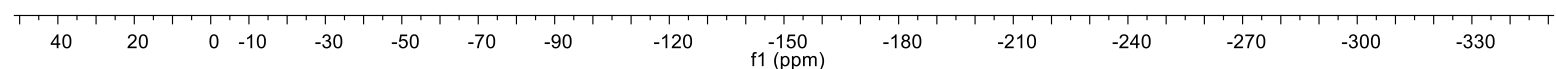

$376 \mathrm{MHz}{ }^{19} \mathrm{~F}$ NMR spectra of compound $3 \mathbf{a a}$ in $\mathrm{CDCl}_{3}$ 

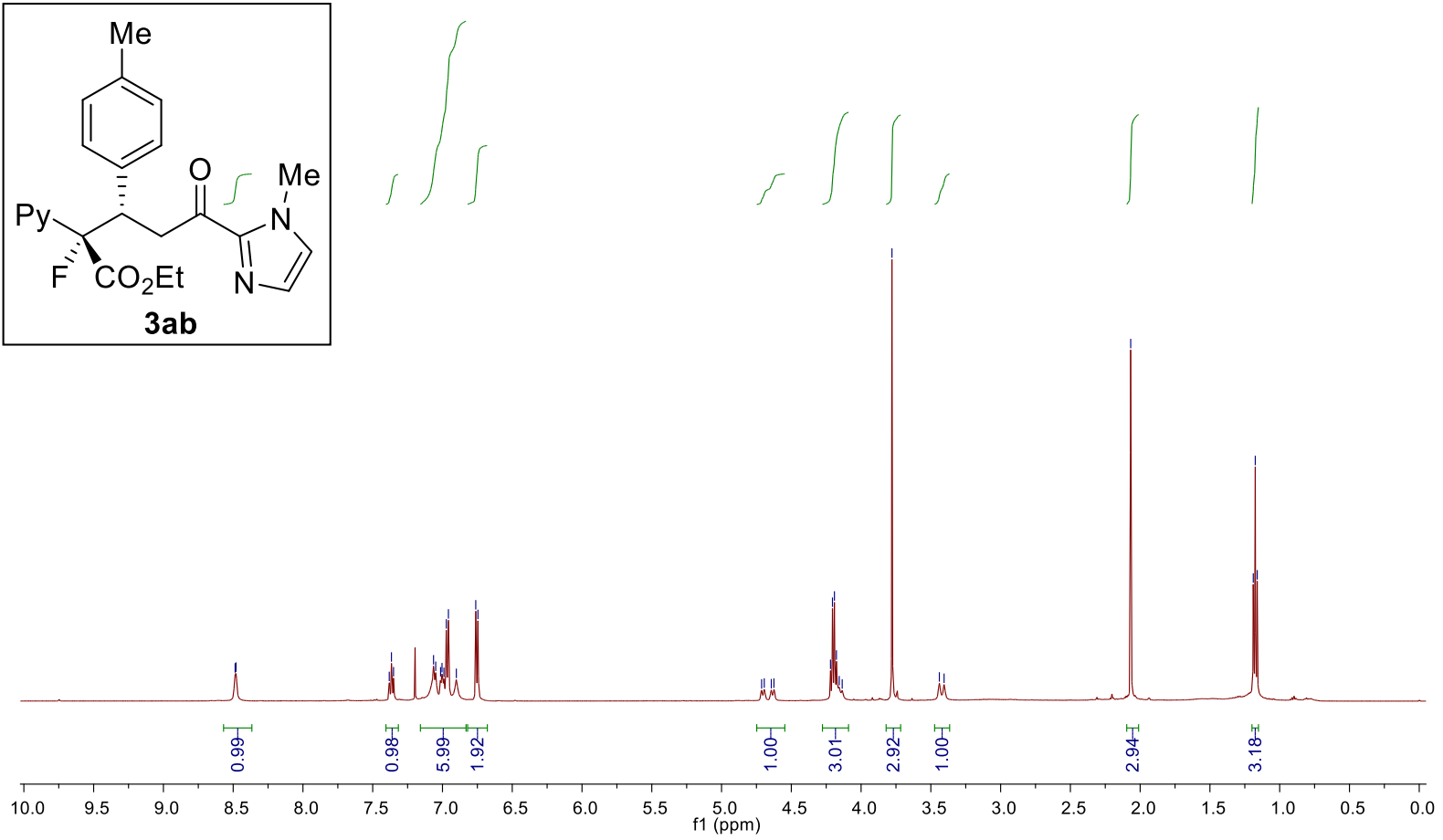

$500 \mathrm{MHz}{ }^{1} \mathrm{H}$ NMR spectra of compound $3 \mathrm{ab}$ in $\mathrm{CDCl}_{3}$

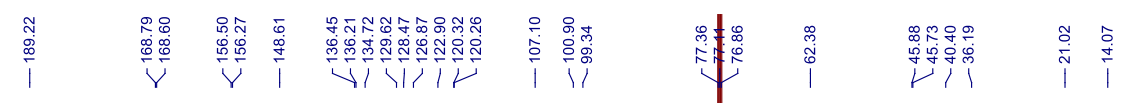<smiles>CCOC(=O)C(CC(=O)c1nccn1C)[C@H](C(=O)OCC)c1ccc(C)cc1</smiles>

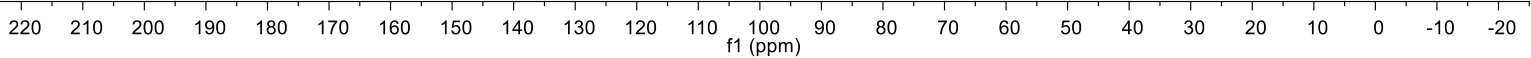
$125 \mathrm{MHz}{ }^{13} \mathrm{C}$ NMR spectra of compound $3 \mathbf{a b}$ in $\mathrm{CDCl}_{3}$ 

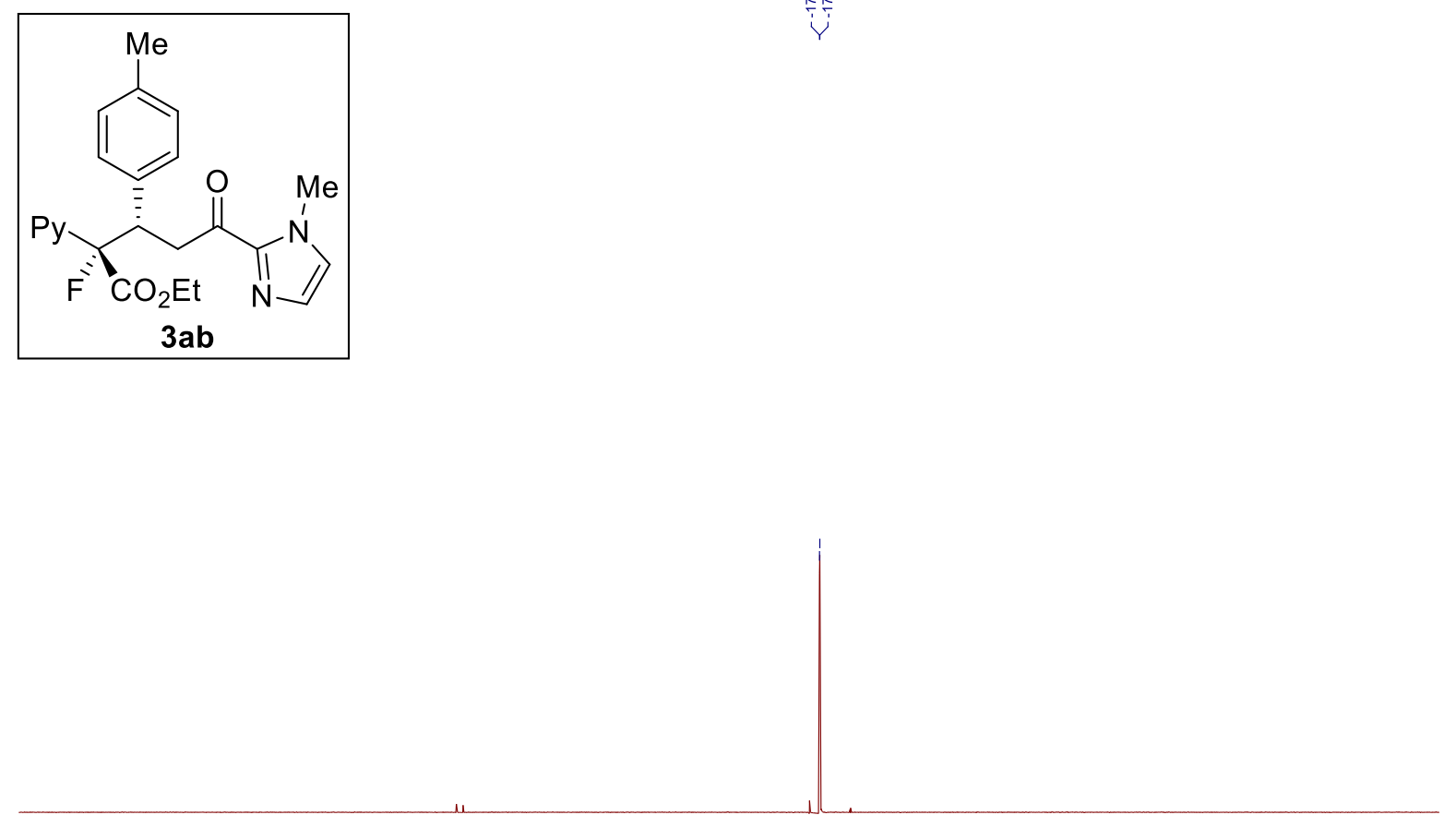

$\begin{array}{lllllllllllllllll} & \\ 40 & 20 & 0 & -10 & -30 & -50 & -70 & -90 & -120 & -150 & -180 & -210 & -240 & -270 & -300 & -330 & \end{array}$

$376 \mathrm{MHz}^{19} \mathrm{~F}$ NMR spectra of compound $3 \mathbf{a b}$ in $\mathrm{CDCl}_{3}$ 

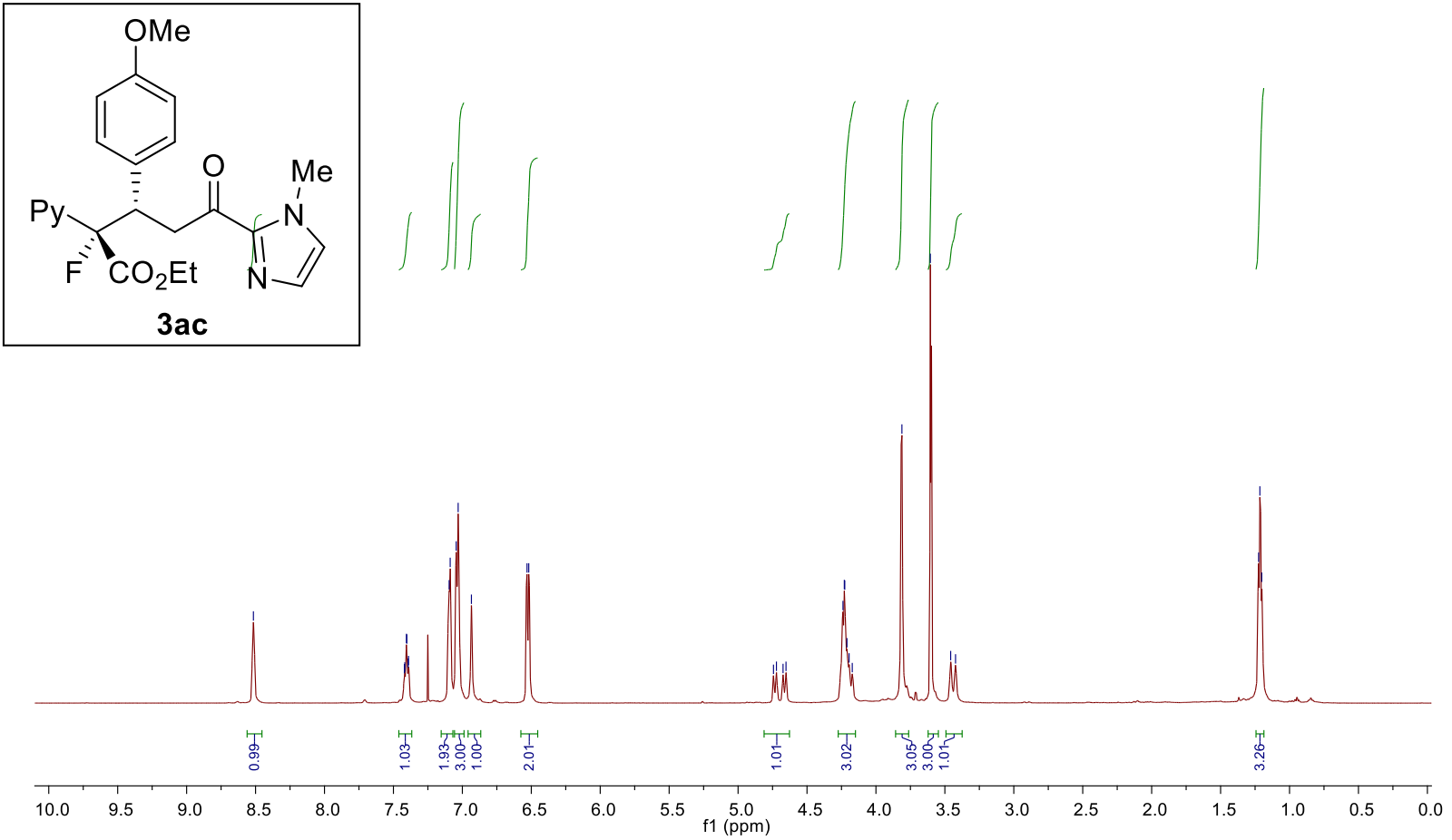

$500 \mathrm{MHz}{ }^{1} \mathrm{H}$ NMR spectra of compound 3ac in $\mathrm{CDCl}_{3}$

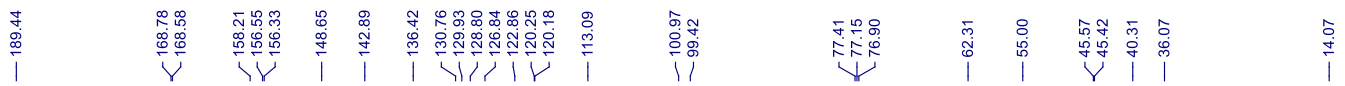
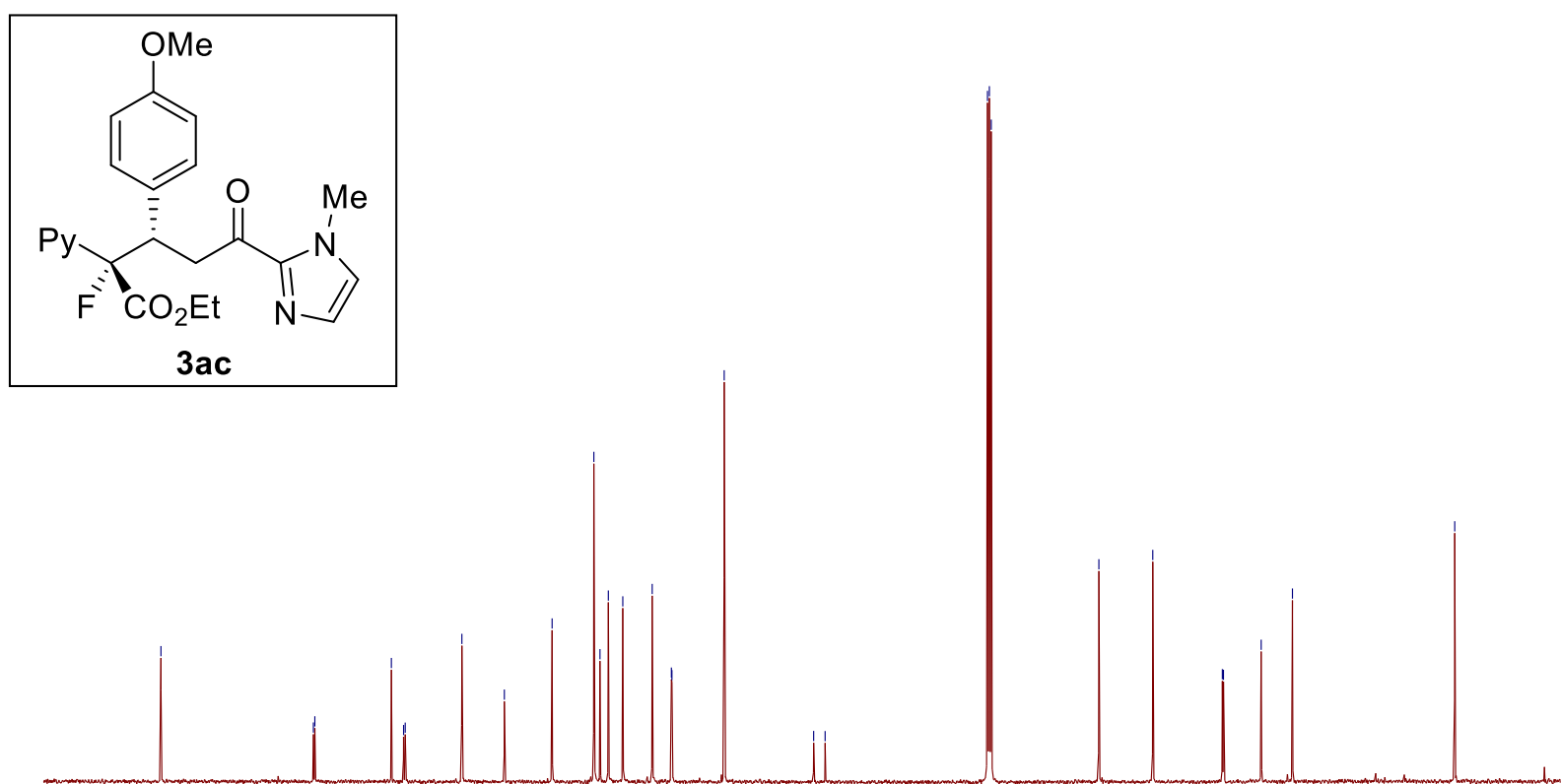

$\begin{array}{llllllllll}200 & 190 & 180 & 170 & 160 & 150 & 140 & 130 & 120 & 110 \\ 100\end{array}$

$125 \mathrm{MHz}{ }^{13} \mathrm{C}$ NMR spectra of compound 3ac in $\mathrm{CDCl}_{3}$ 

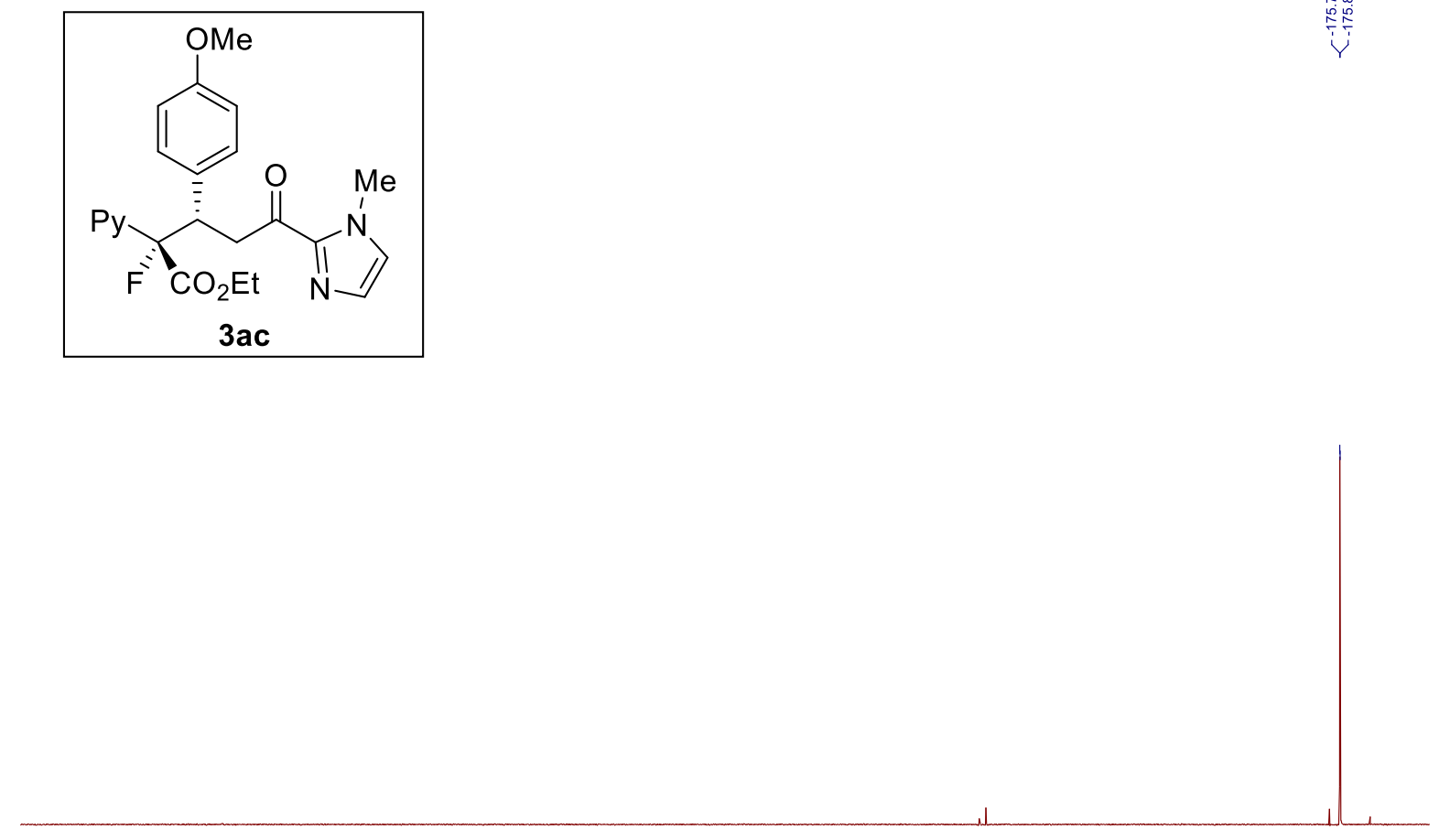

$\begin{array}{llllllllllllllllllllll} & 180 & 160 & 140 & 120 & 100 & 80 & 60 & 40 & 20 & 0 & -10 & -30 & -50 & -70 & -90 & -120 & -150 & -180\end{array}$

$376 \mathrm{MHz}{ }^{19} \mathrm{~F}$ NMR spectra of compound 3ac in $\mathrm{CDCl}_{3}$ 

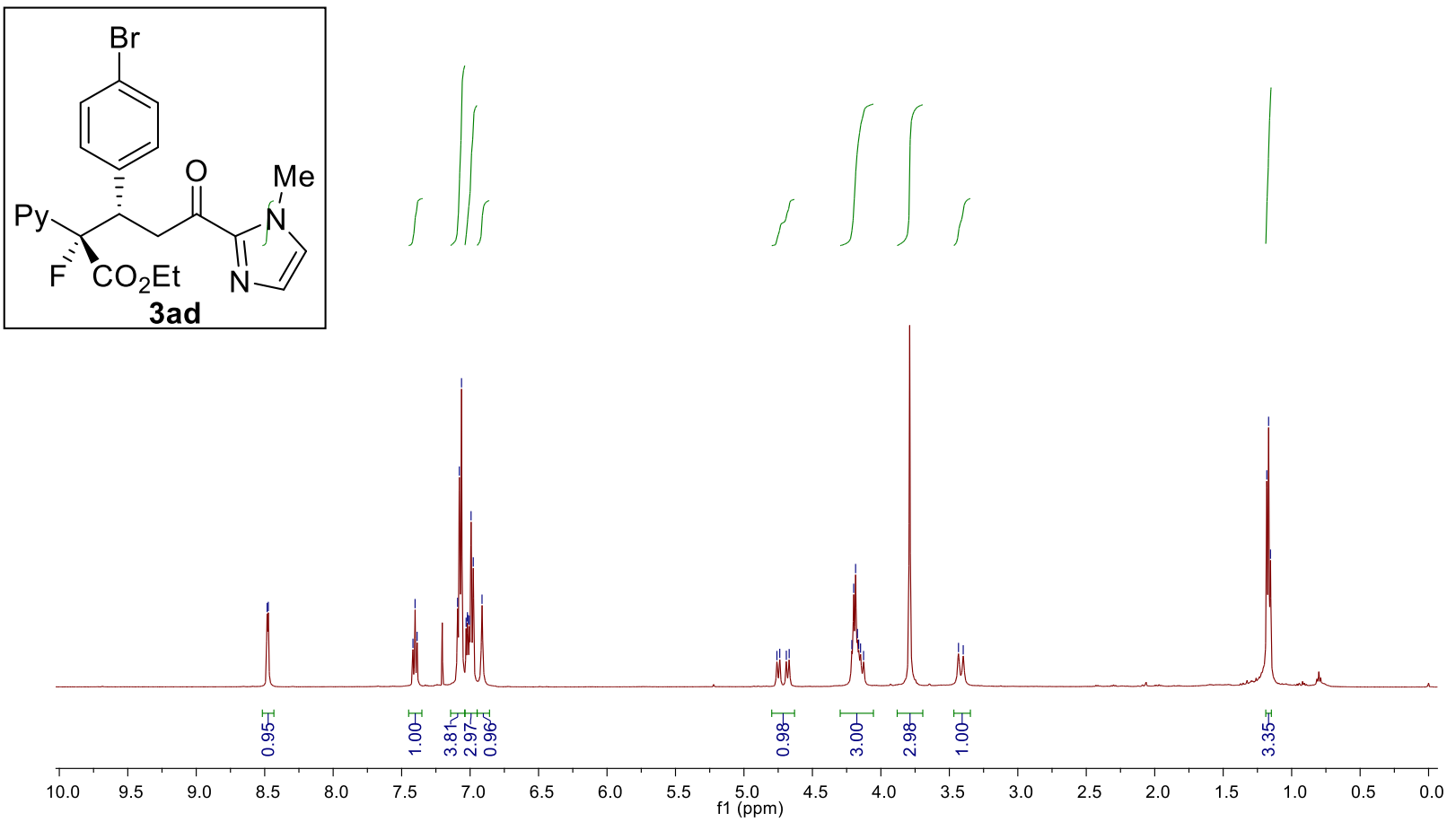

$500 \mathrm{MHz}{ }^{1} \mathrm{H}$ NMR spectra of compound 3ad in $\mathrm{CDCl}_{3}$

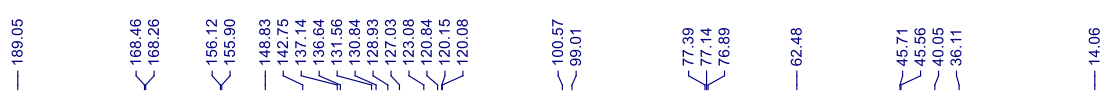
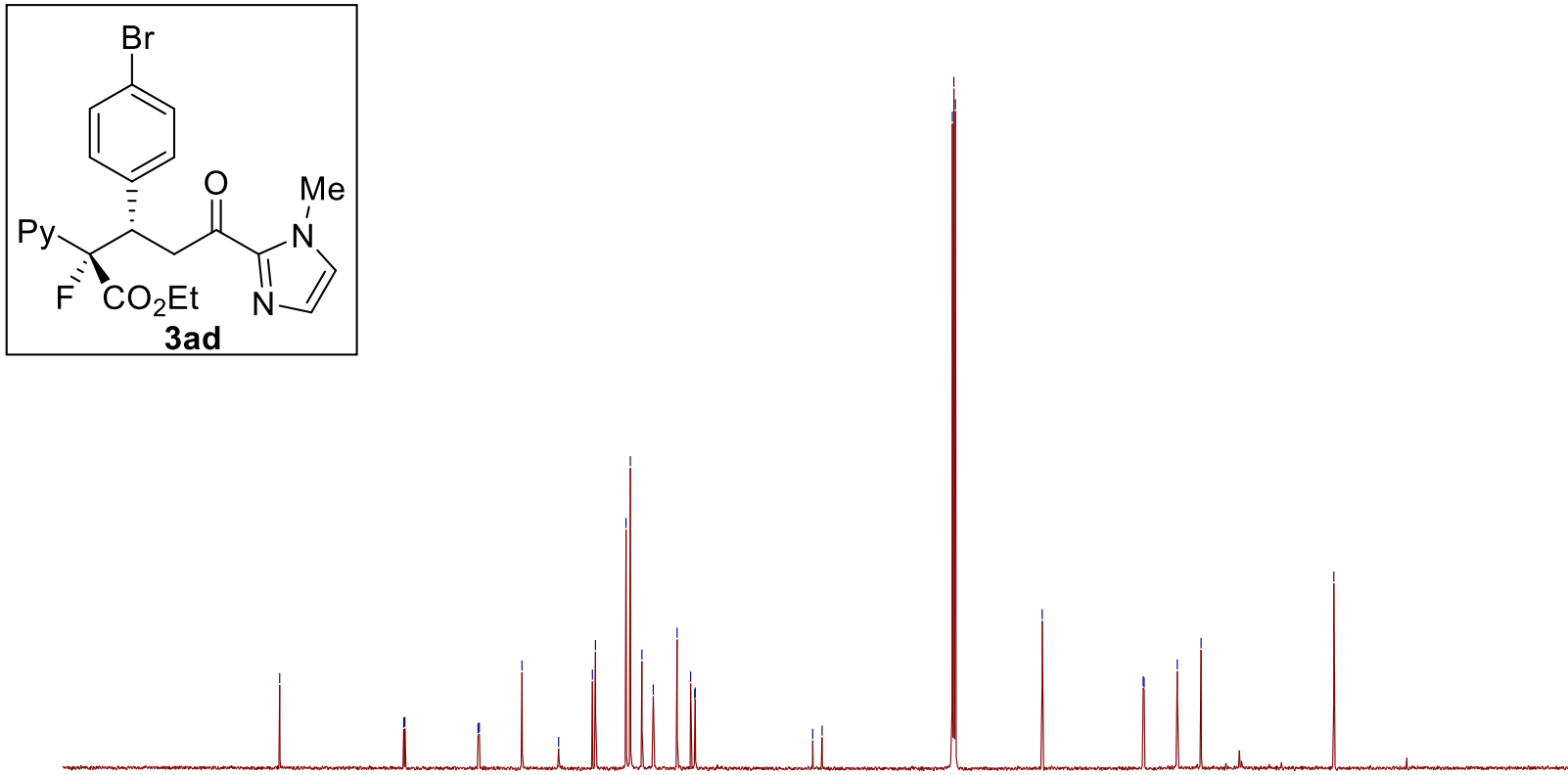

$\begin{array}{lllllllllllllllllllllllllllllll}220 & 210 & 200 & 190 & 180 & 170 & 160 & 150 & 140 & 130 & 120 & 110 & 100 & 90 & 80 & 70 & 60 & 50 & 40 & 30 & 20 & 10 & 0 & -10 & -20\end{array}$ $125 \mathrm{MHz}{ }^{13} \mathrm{C}$ NMR spectra of compound 3ad in $\mathrm{CDCl}_{3}$ 


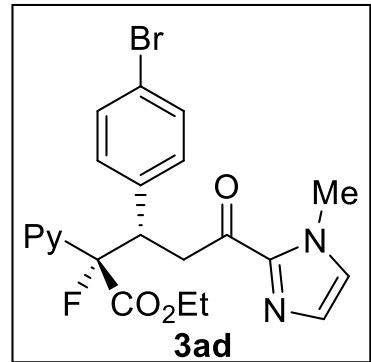

$\begin{array}{llllllllllllllll} & \\ 40 & 20 & 0 & -10 & -30 & -50 & -70 & -90 & -120 & -150 & -180 & -210 & -240 & -270 & -300 & -330\end{array}$

$376 \mathrm{MHz}^{19} \mathrm{~F}$ NMR spectra of compound $3 \mathrm{ad}$ in $\mathrm{CDCl}_{3}$ 

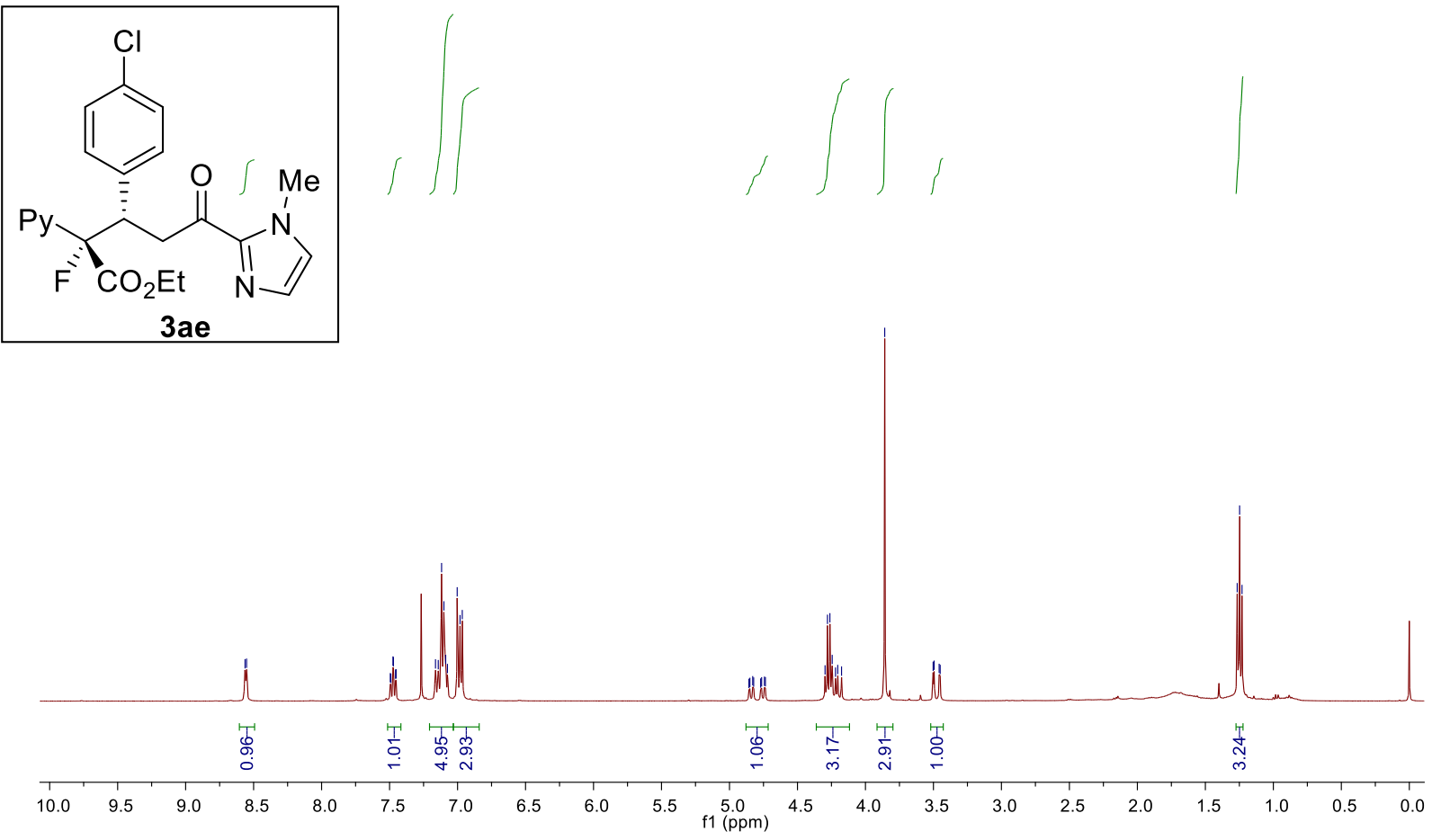

$400 \mathrm{MHz}{ }^{1} \mathrm{H}$ NMR spectra of compound $3 \mathrm{ae}$ in $\mathrm{CDCl}_{3}$

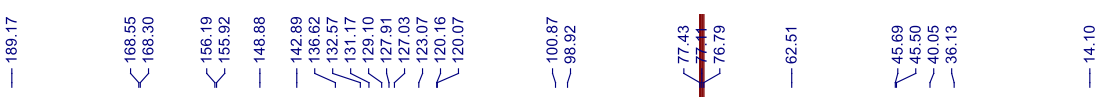<smiles>CCOC[C@@H](c1ccc(Cl)cc1)[C@@](C)(F)CC(=O)c1nccn1C</smiles>

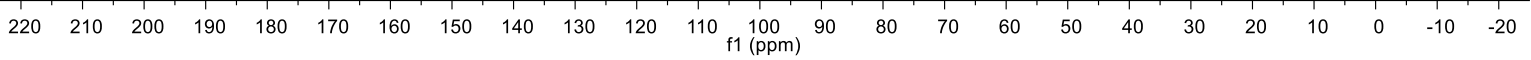
$100 \mathrm{MHz}{ }^{13} \mathrm{C}$ NMR spectra of compound 3ae in $\mathrm{CDCl}_{3}$ 


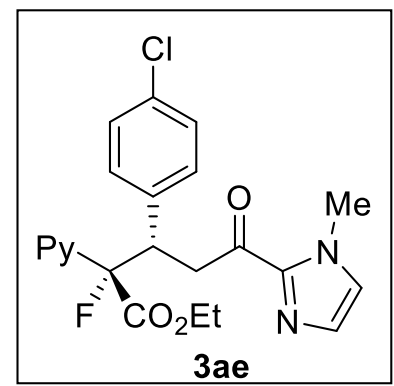

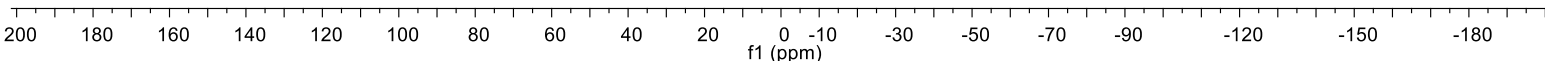

$376 \mathrm{MHz}^{19} \mathrm{~F}$ NMR spectra of compound 3ae in $\mathrm{CDCl}_{3}$ 


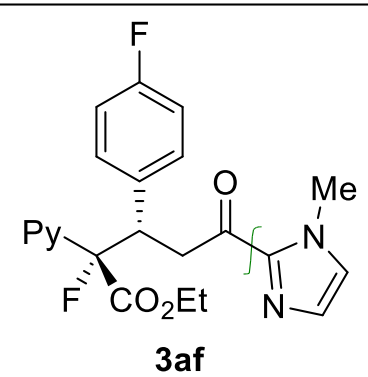

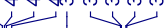
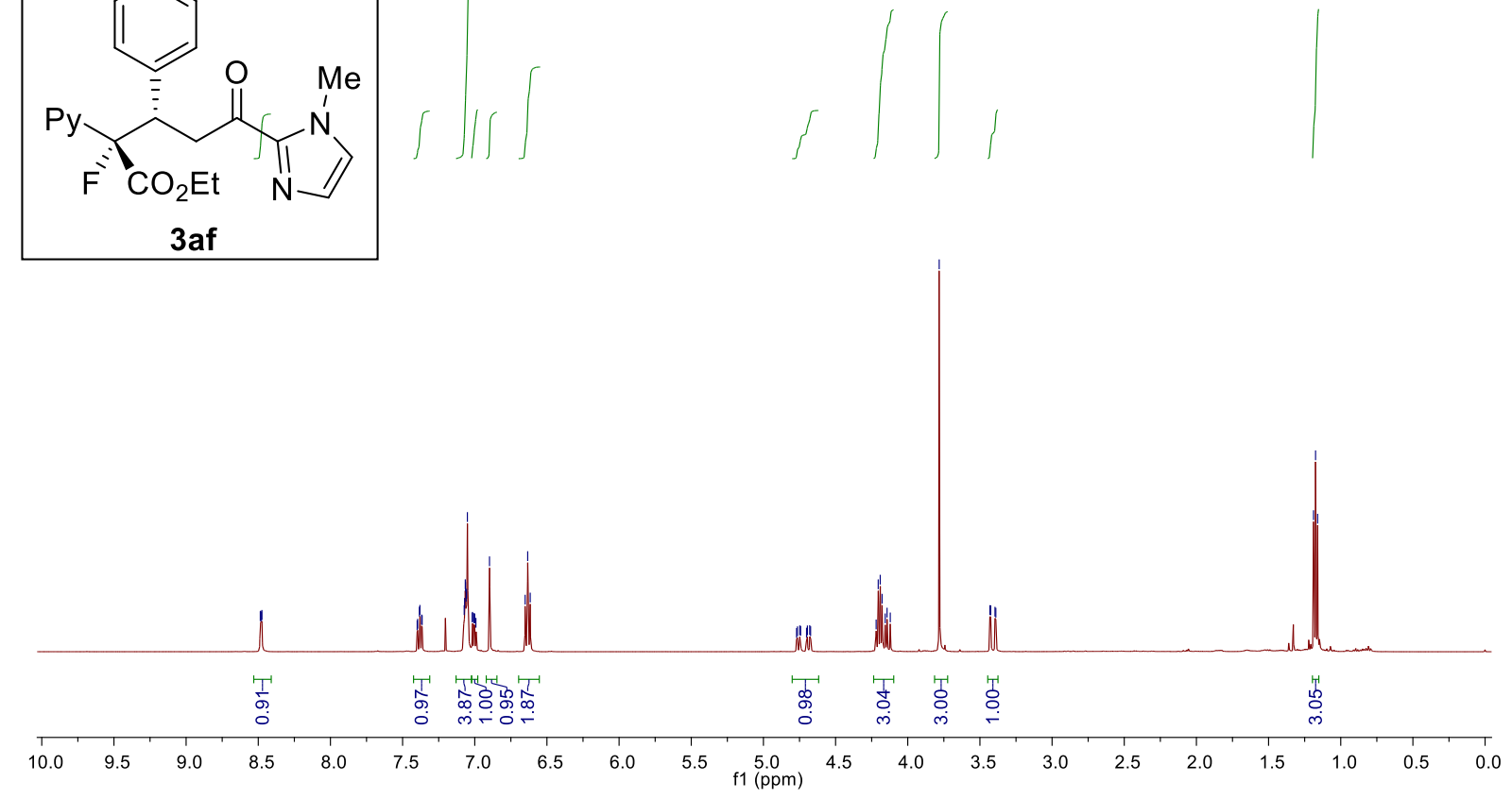

$500 \mathrm{MHz}{ }^{1} \mathrm{H}$ NMR spectra of compound 3af in $\mathrm{CDCl}_{3}$

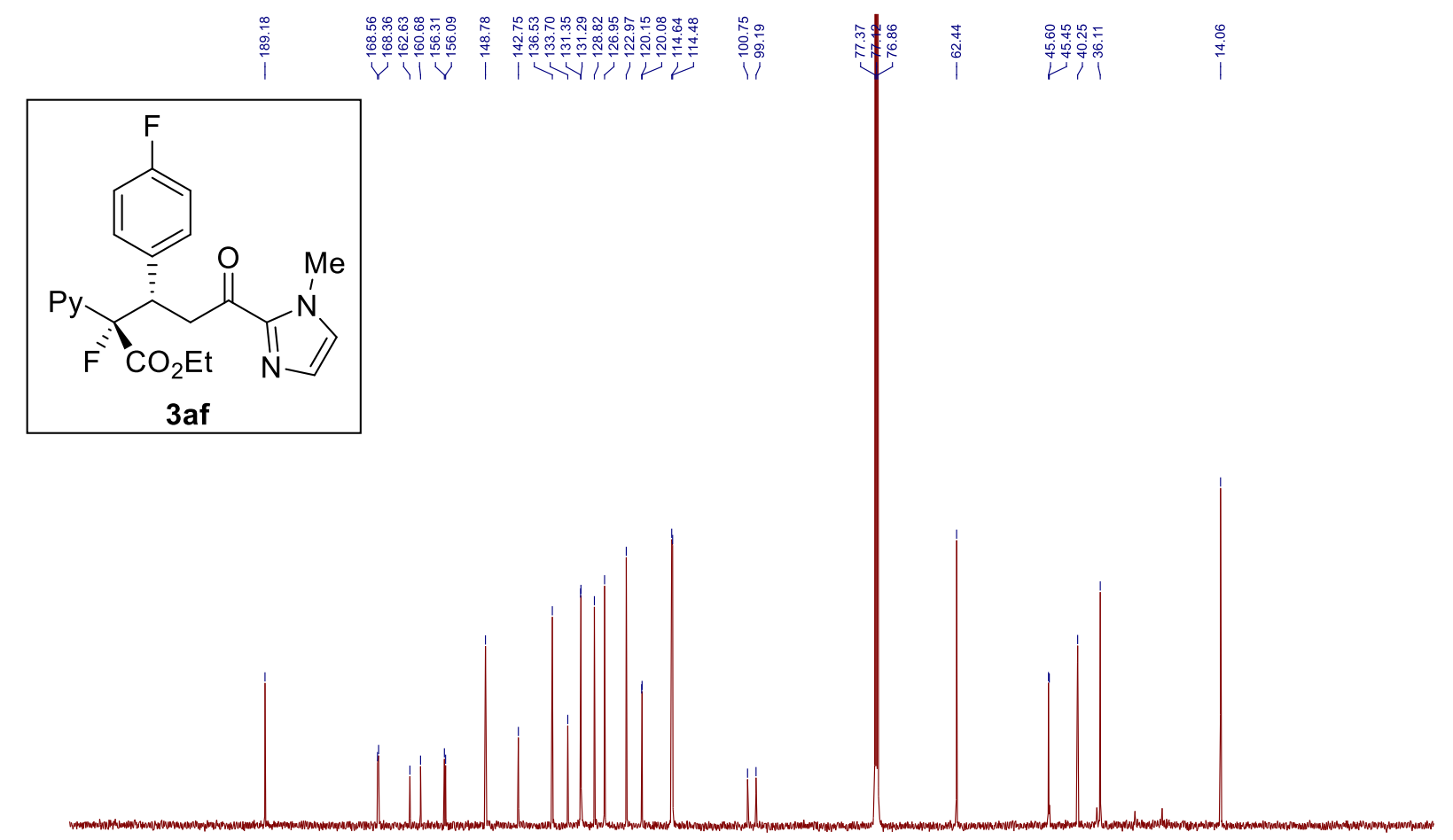

$\begin{array}{lllllllllllllllllllllllll}220 & 210 & 200 & 190 & 180 & 170 & 160 & 150 & 140 & 130 & 120 & 110 & 100 & 90 & 80 & 70 & 60 & 50 & 40 & 30 & 20 & 10 & 0 & -10 & -20\end{array}$ $125 \mathrm{MHz}{ }^{13} \mathrm{C}$ NMR spectra of compound 3af in $\mathrm{CDCl}_{3}$ 


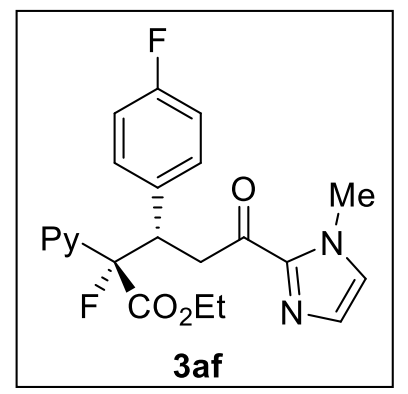

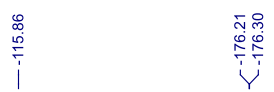

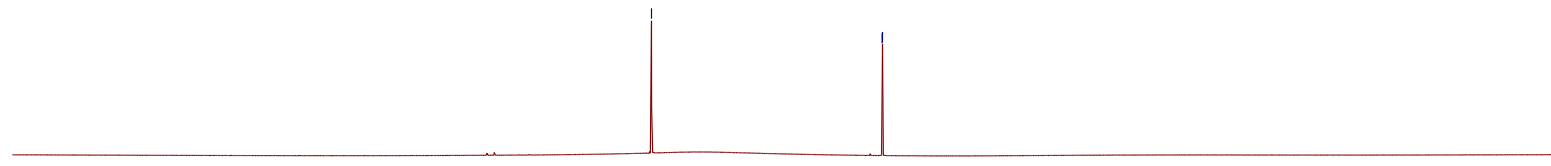

$\begin{array}{lllllllllllllllll} & \\ 40 & 20 & 0 & -10 & -30 & -50 & -70 & -90 & -120 & -150 & -180 & -210 & -240 & -270 & -300 & -330 & \end{array}$

$376 \mathrm{MHz}{ }^{19} \mathrm{~F}$ NMR spectra of compound 3af in $\mathrm{CDCl}_{3}$ 

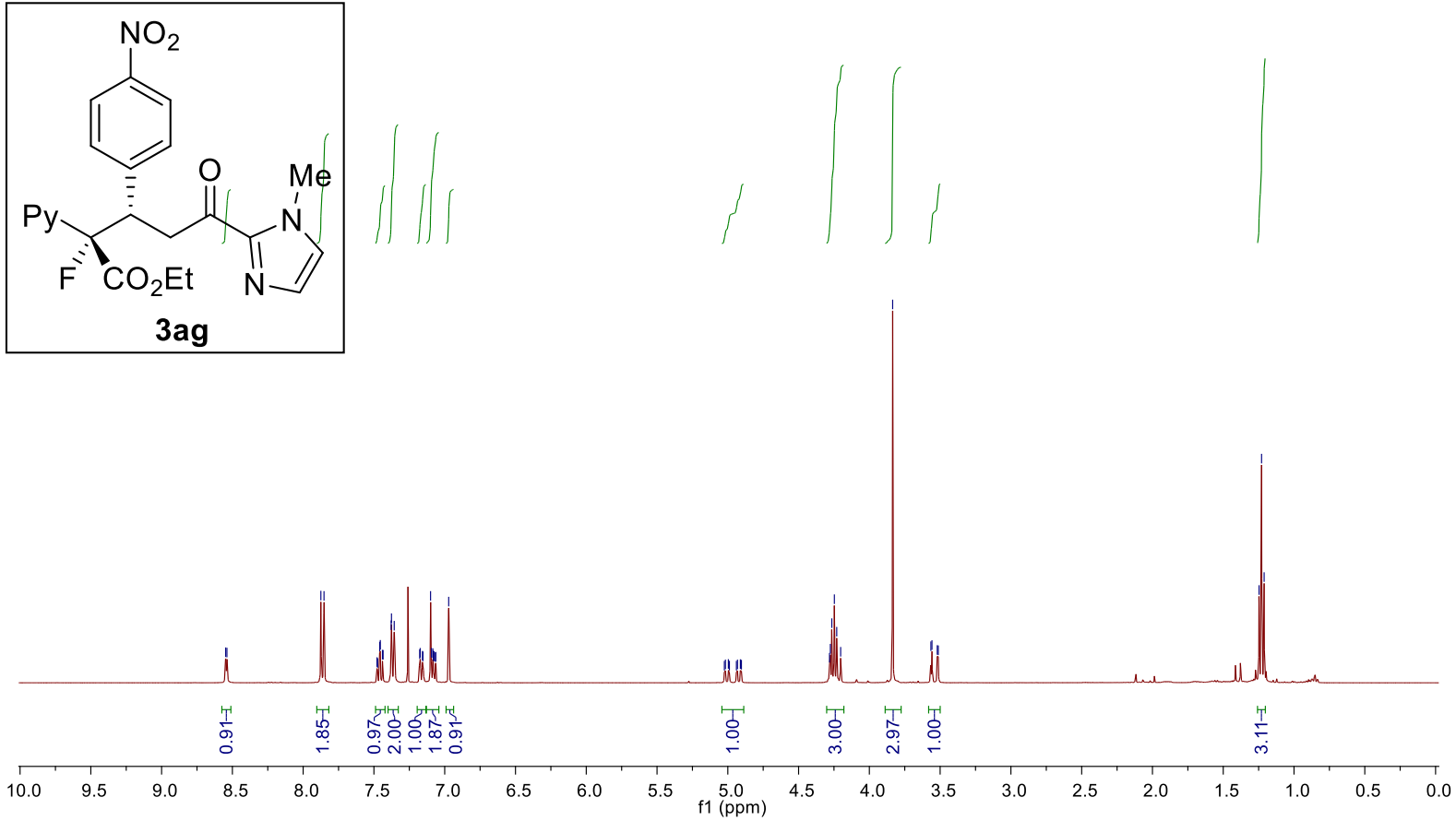

$400 \mathrm{MHz}{ }^{1} \mathrm{H}$ NMR spectra of compound 3ag in $\mathrm{CDCl}_{3}$

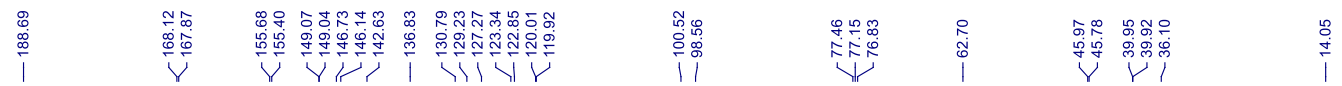
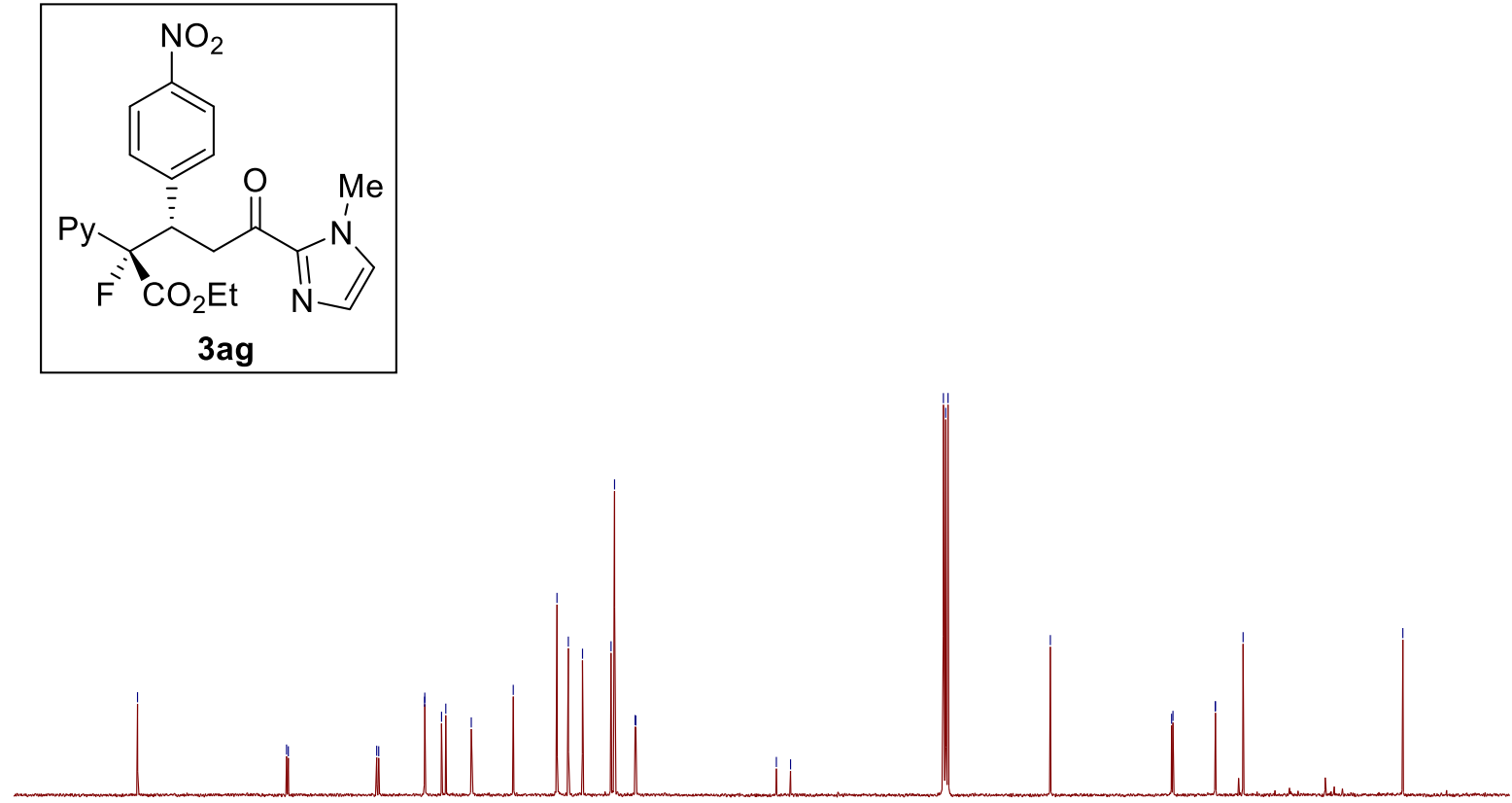

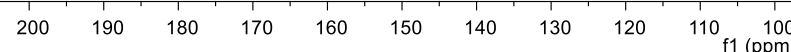
$100 \mathrm{MHz}{ }^{13} \mathrm{C}$ NMR spectra of compound 3 ag in $\mathrm{CDCl}_{3}$ 

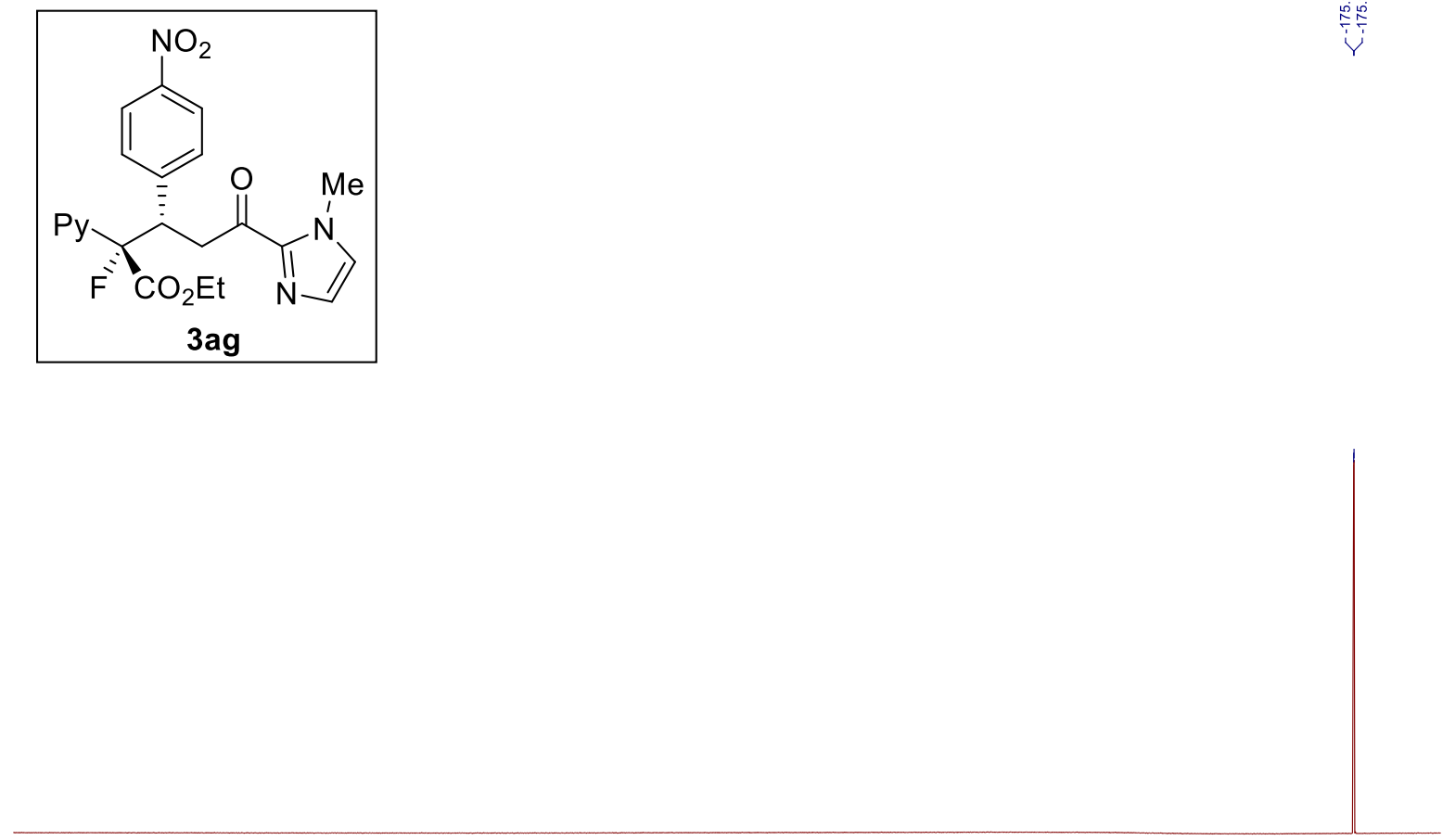

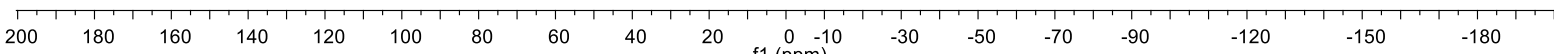

$376 \mathrm{MHz}^{19} \mathrm{~F}$ NMR spectra of compound $3 \mathrm{ag}$ in $\mathrm{CDCl}_{3}$ 


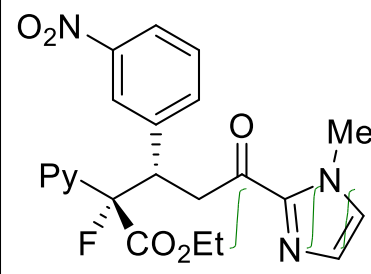

3ah

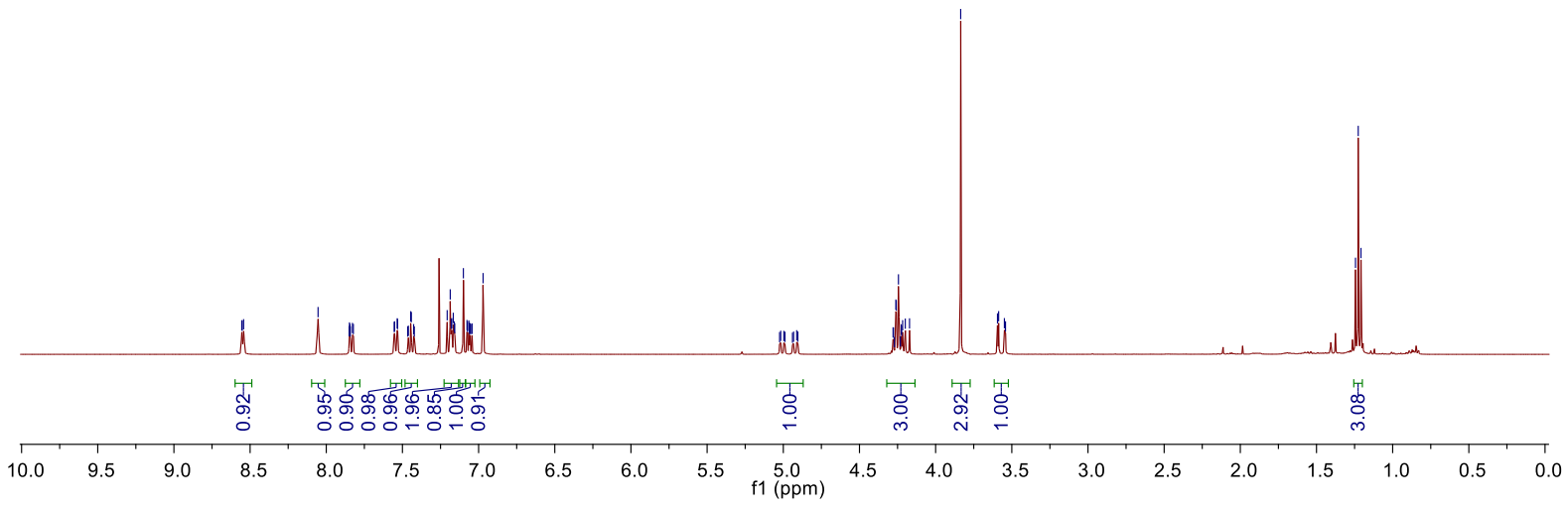
$400 \mathrm{MHz}{ }^{1} \mathrm{H}$ NMR spectra of compound 3ah in $\mathrm{CDCl}_{3}$

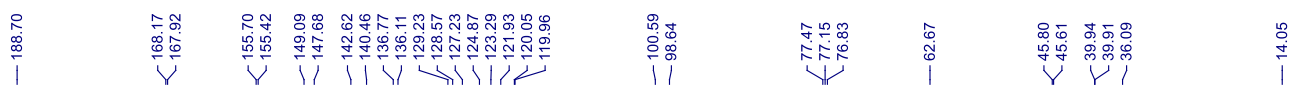
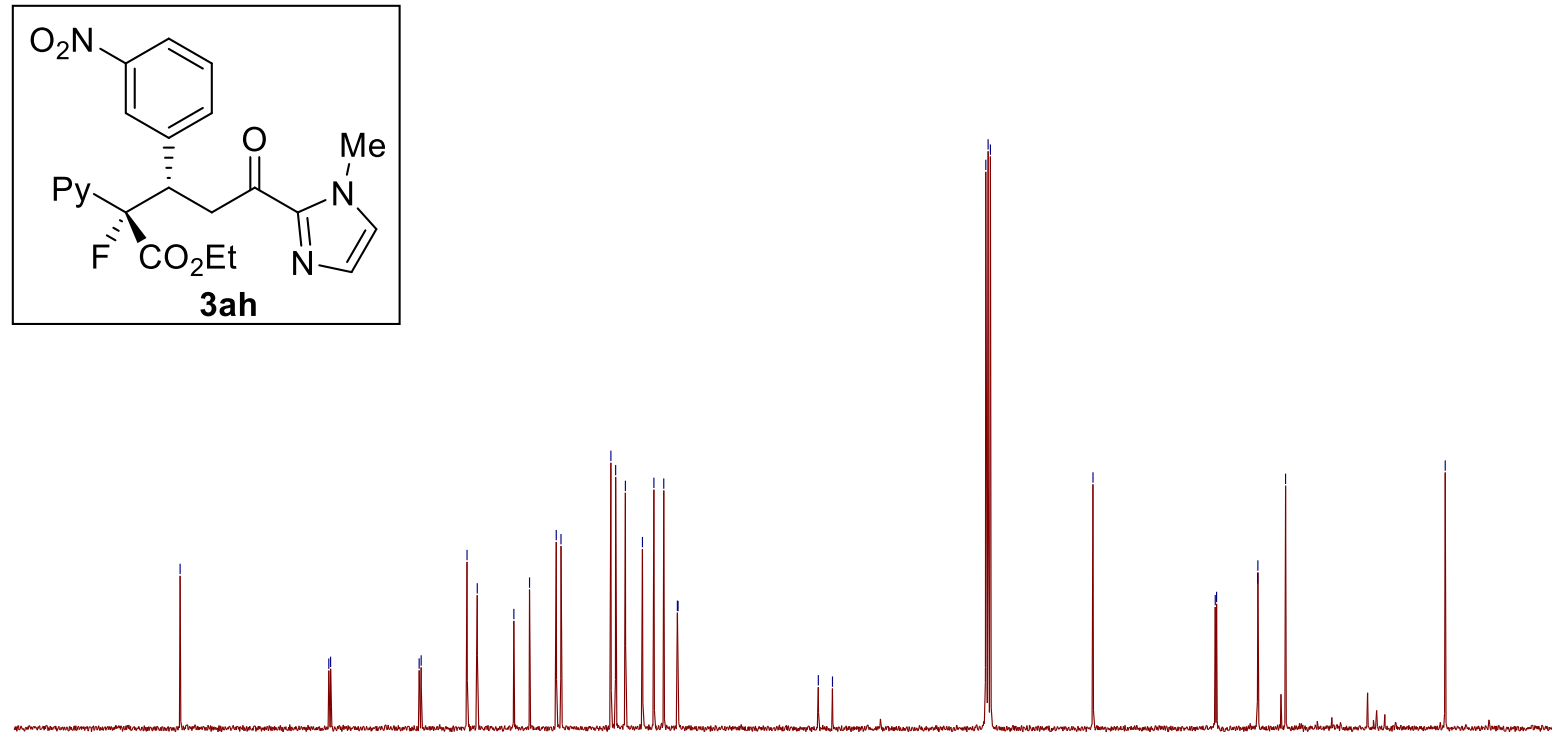

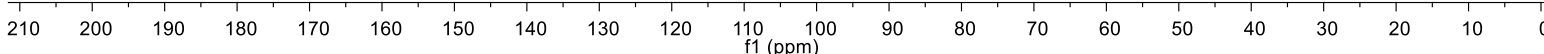
$100 \mathrm{MHz}{ }^{13} \mathrm{C}$ NMR spectra of compound 3ah in $\mathrm{CDCl}_{3}$ 

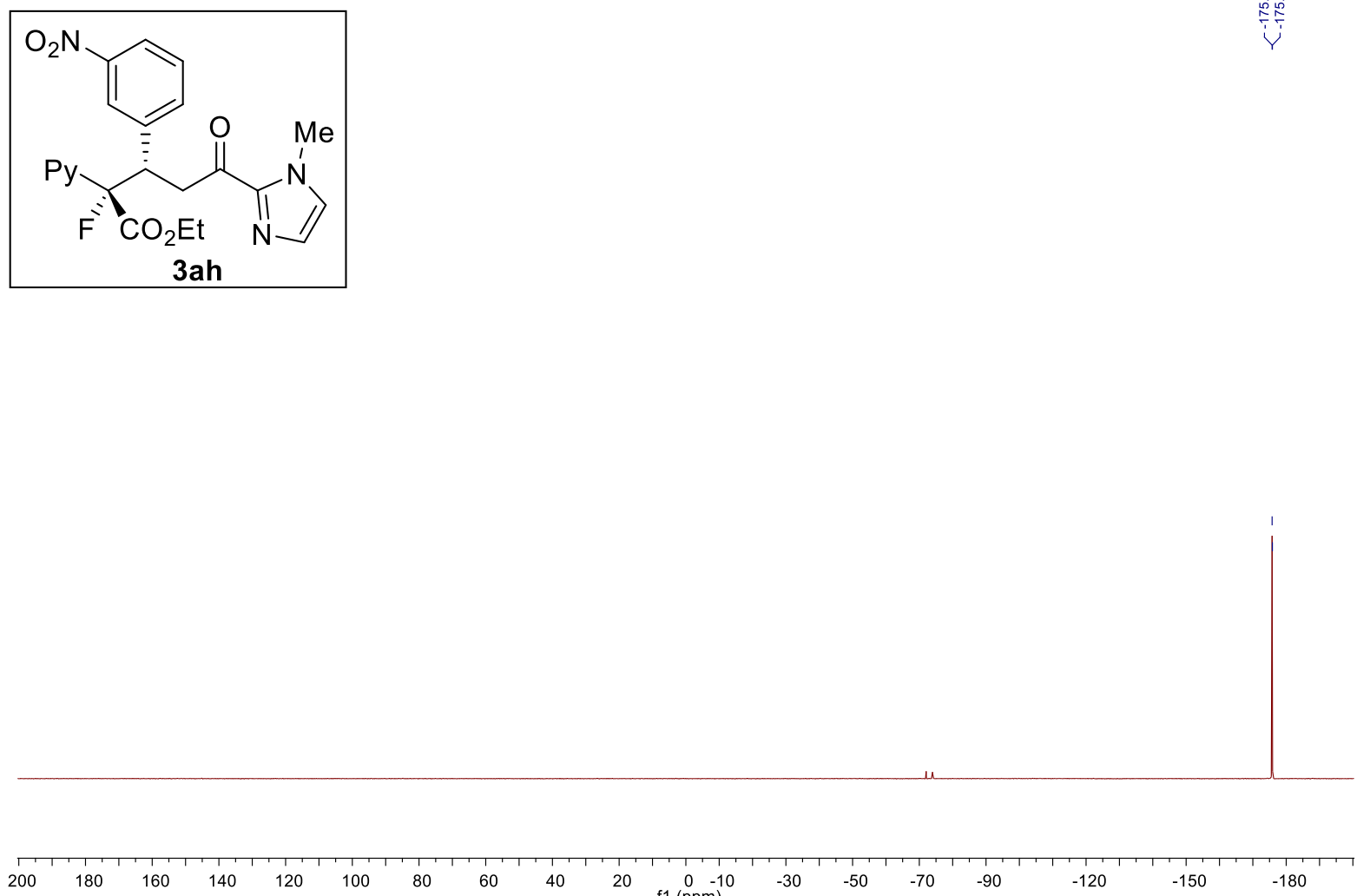

$376 \mathrm{MHz}{ }^{19} \mathrm{~F}$ NMR spectra of compound $3 \mathrm{ah}$ in $\mathrm{CDCl}_{3}$ 


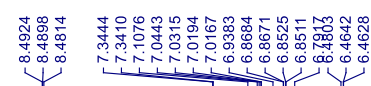

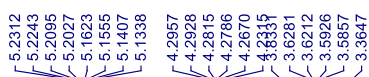
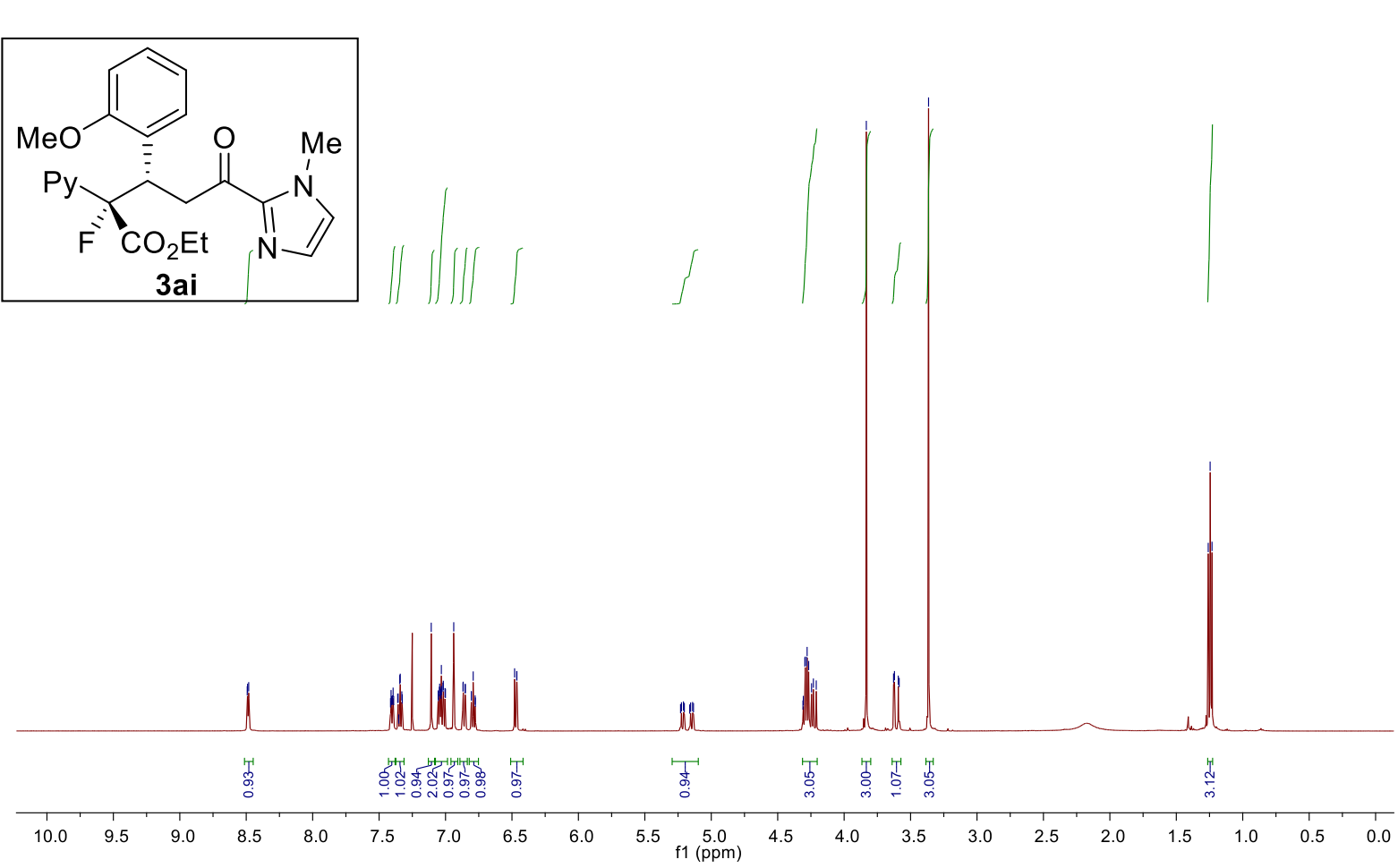

$500 \mathrm{MHz}{ }^{1} \mathrm{H}$ NMR spectra of compound 3ai in $\mathrm{CDCl}_{3}$

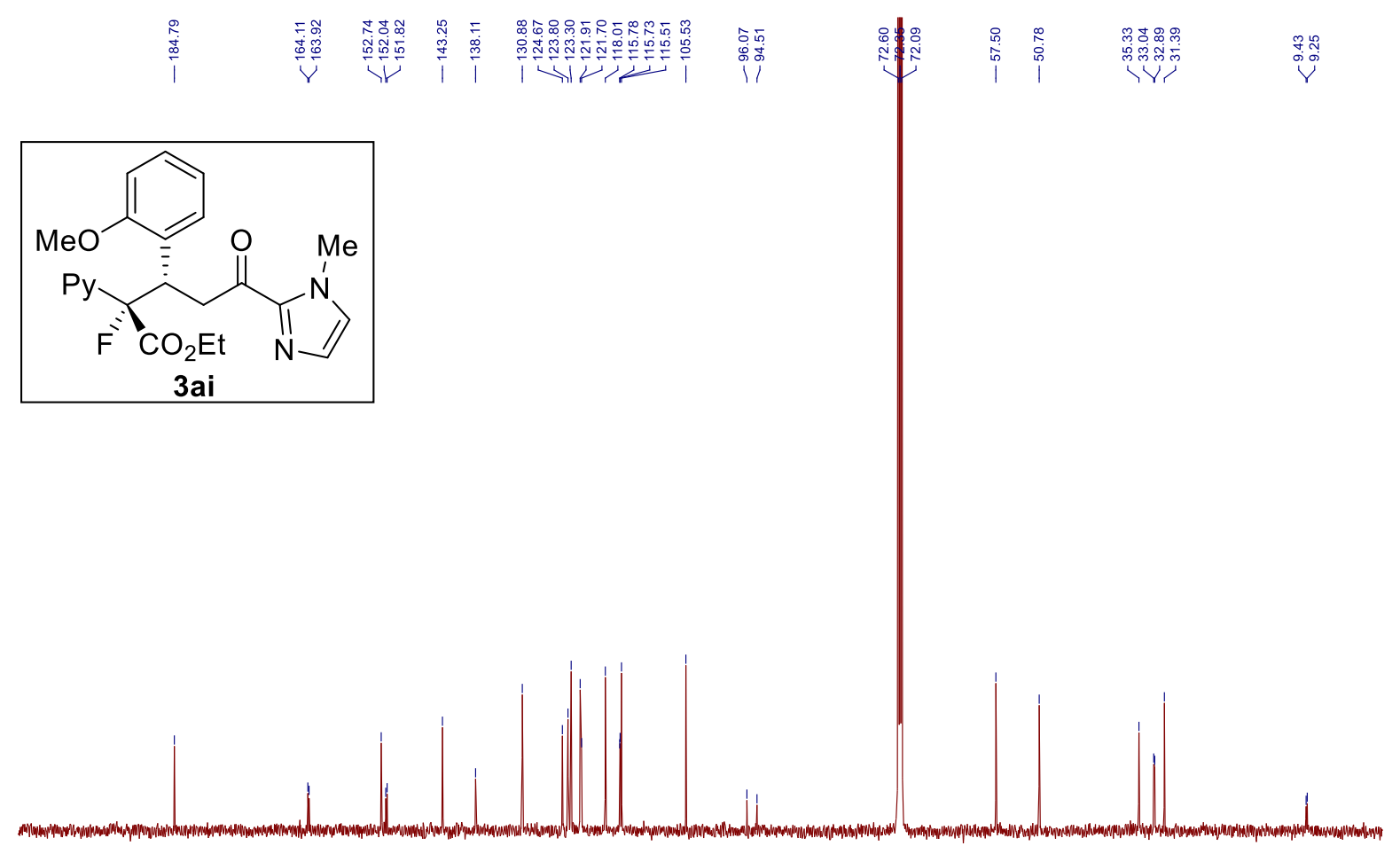

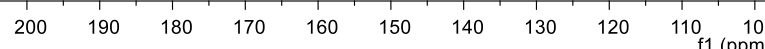

$125 \mathrm{MHz}{ }^{13} \mathrm{C}$ NMR spectra of compound 3ai in $\mathrm{CDCl}_{3}$ 


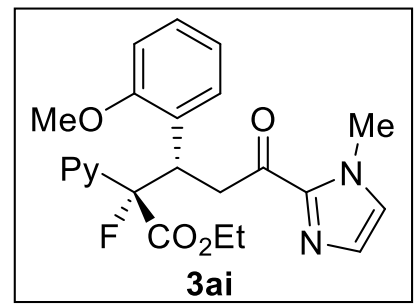

$376 \mathrm{MHz}{ }^{19} \mathrm{~F}$ NMR spectra of compound 3ai in $\mathrm{CDCl}_{3}$ 
<smiles>CCOC(=O)C(CC(=O)c1nccn1C)[C@H](C(=O)OCC)c1ccccc1Cl</smiles>

3aj

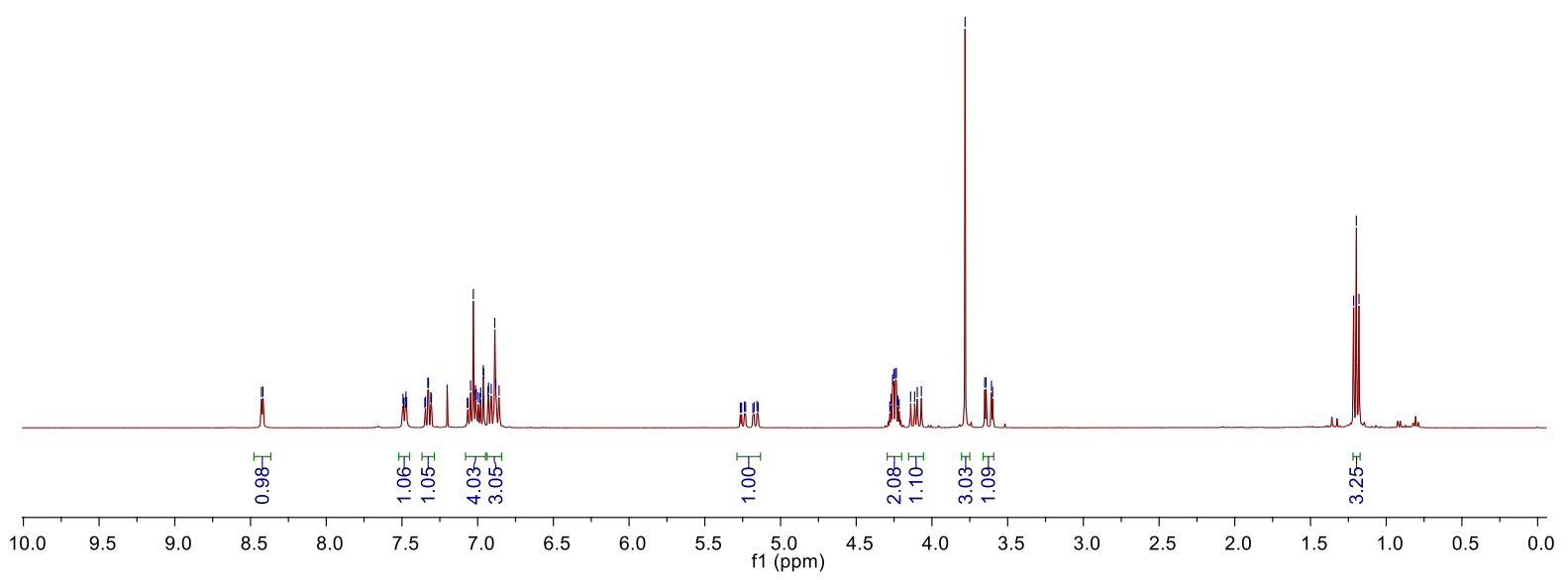

$400 \mathrm{MHz}{ }^{1} \mathrm{H}$ NMR spectra of compound 3aj in $\mathrm{CDCl}_{3}$

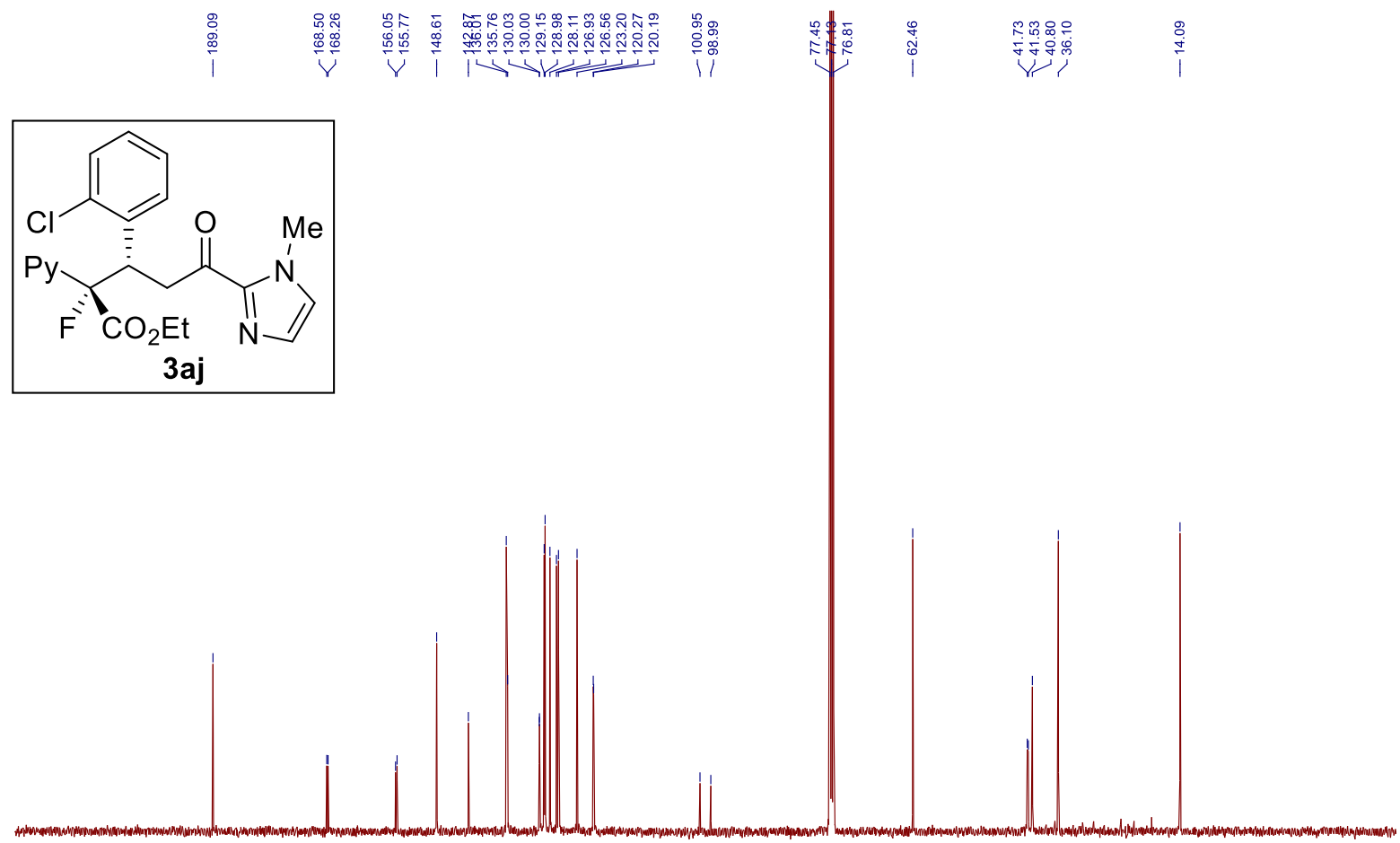

$\begin{array}{lllllllllllllllllllllllll}220 & 210 & 200 & 190 & 180 & 170 & 160 & 150 & 140 & 130 & 120 & 110 & \begin{array}{c}100 \\ \mathrm{f} 1(\mathrm{ppm})\end{array} & 90 & 80 & 70 & 60 & 50 & 40 & 30 & 20 & 10 & 0 & -10 & -20\end{array}$ $100 \mathrm{MHz}{ }^{13} \mathrm{C}$ NMR spectra of compound 3aj in $\mathrm{CDCl}_{3}$ 


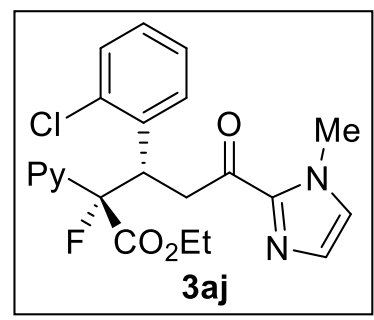

ํํำ

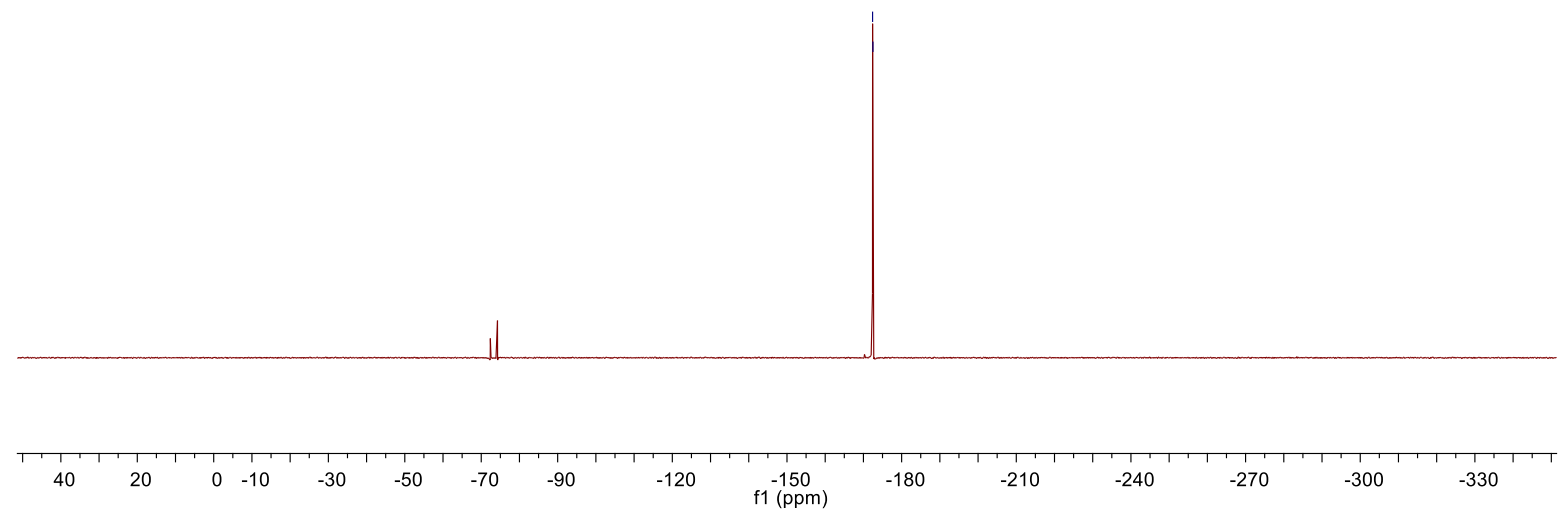

$376 \mathrm{MHz}{ }^{19} \mathrm{~F}$ NMR spectra of compound 3aj in $\mathrm{CDCl}_{3}$ 

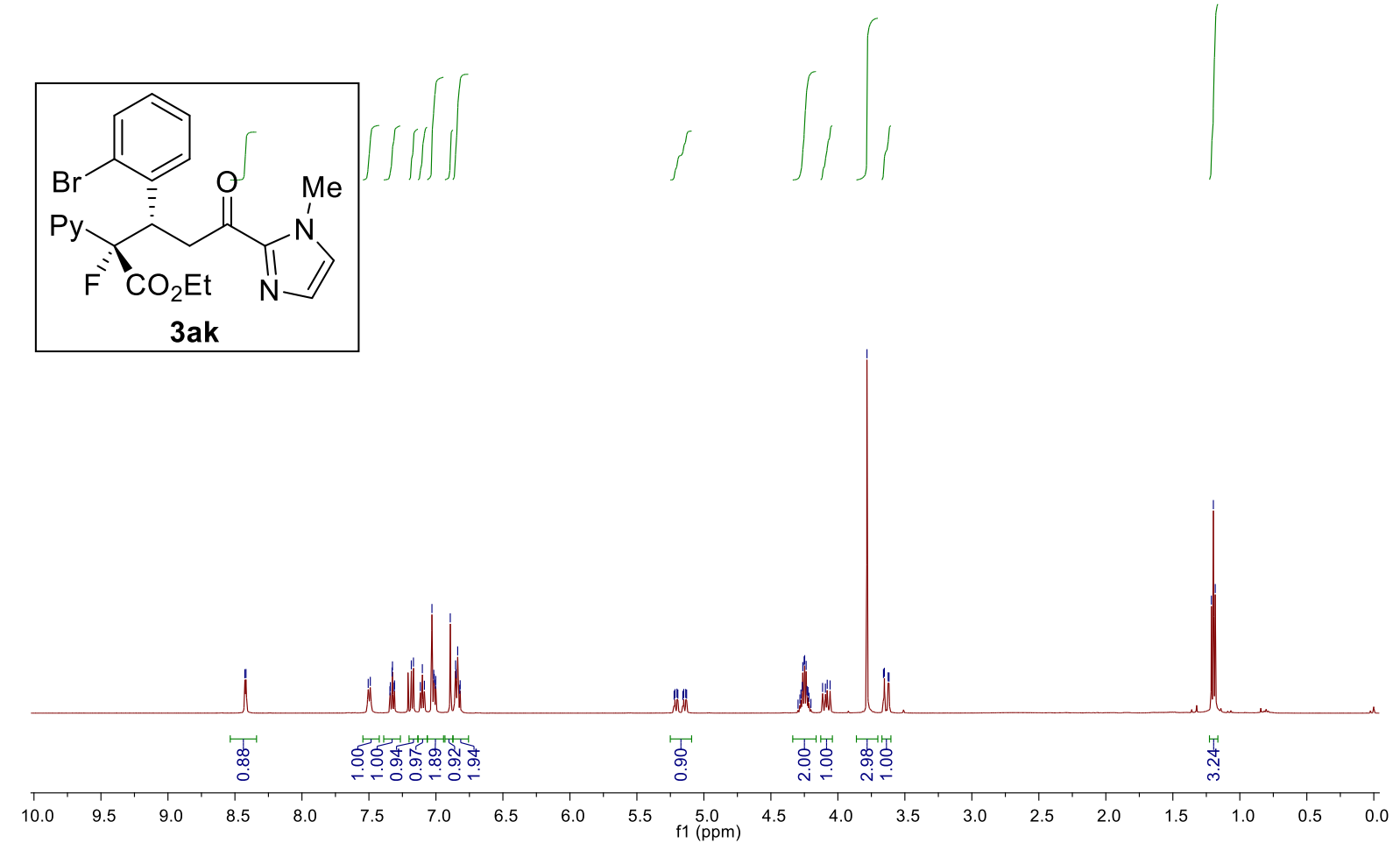

$500 \mathrm{MHz}{ }^{1} \mathrm{H}$ NMR spectra of compound 3ak in $\mathrm{CDCl}_{3}$

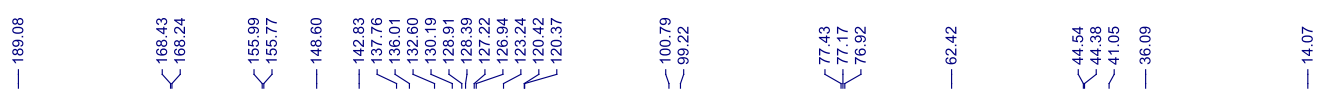
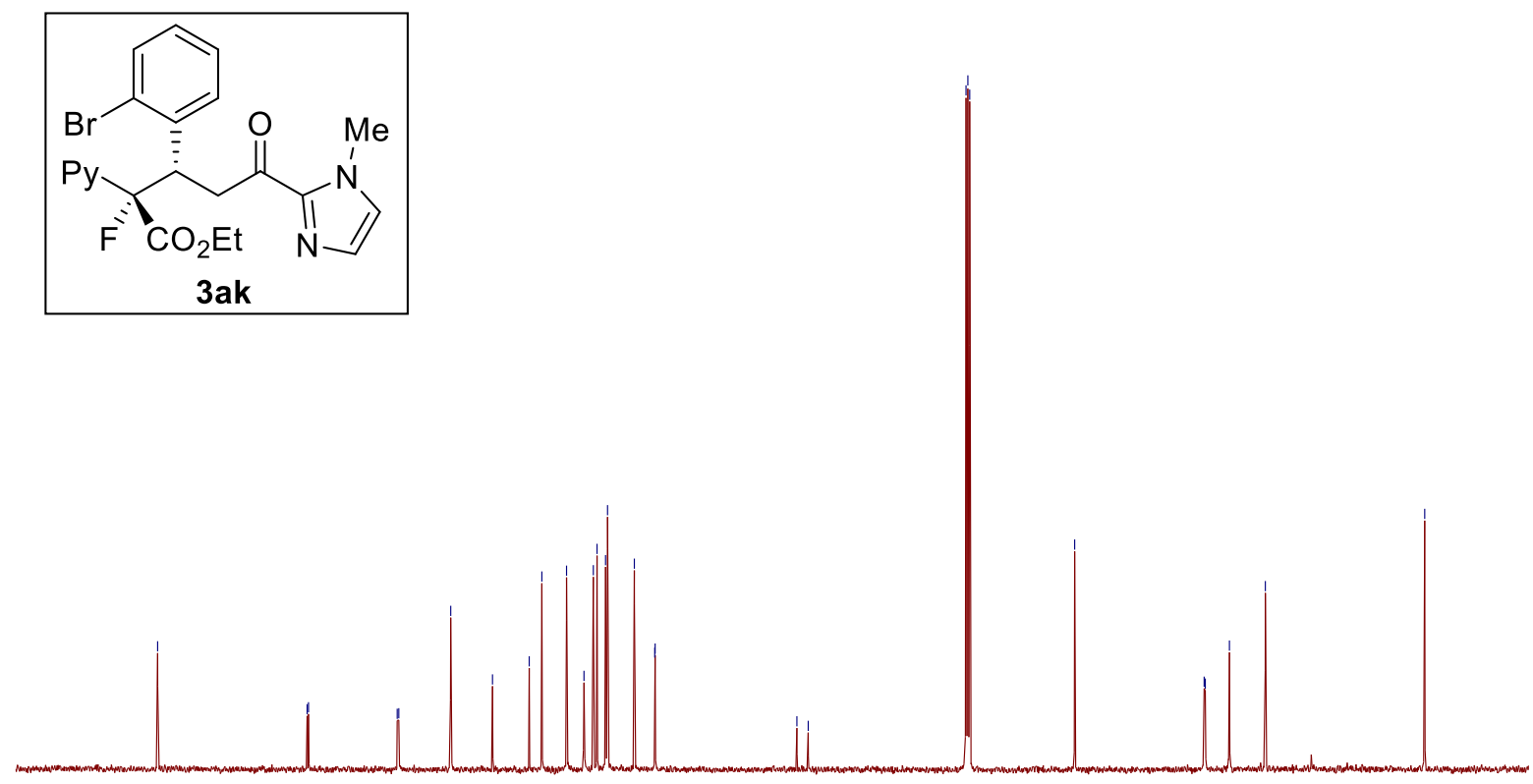

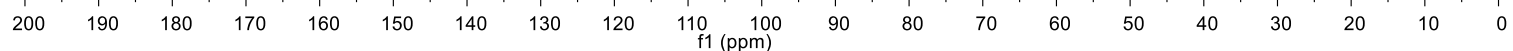

$125 \mathrm{MHz}{ }^{13} \mathrm{C}$ NMR spectra of compound 3ak in $\mathrm{CDCl}_{3}$ 

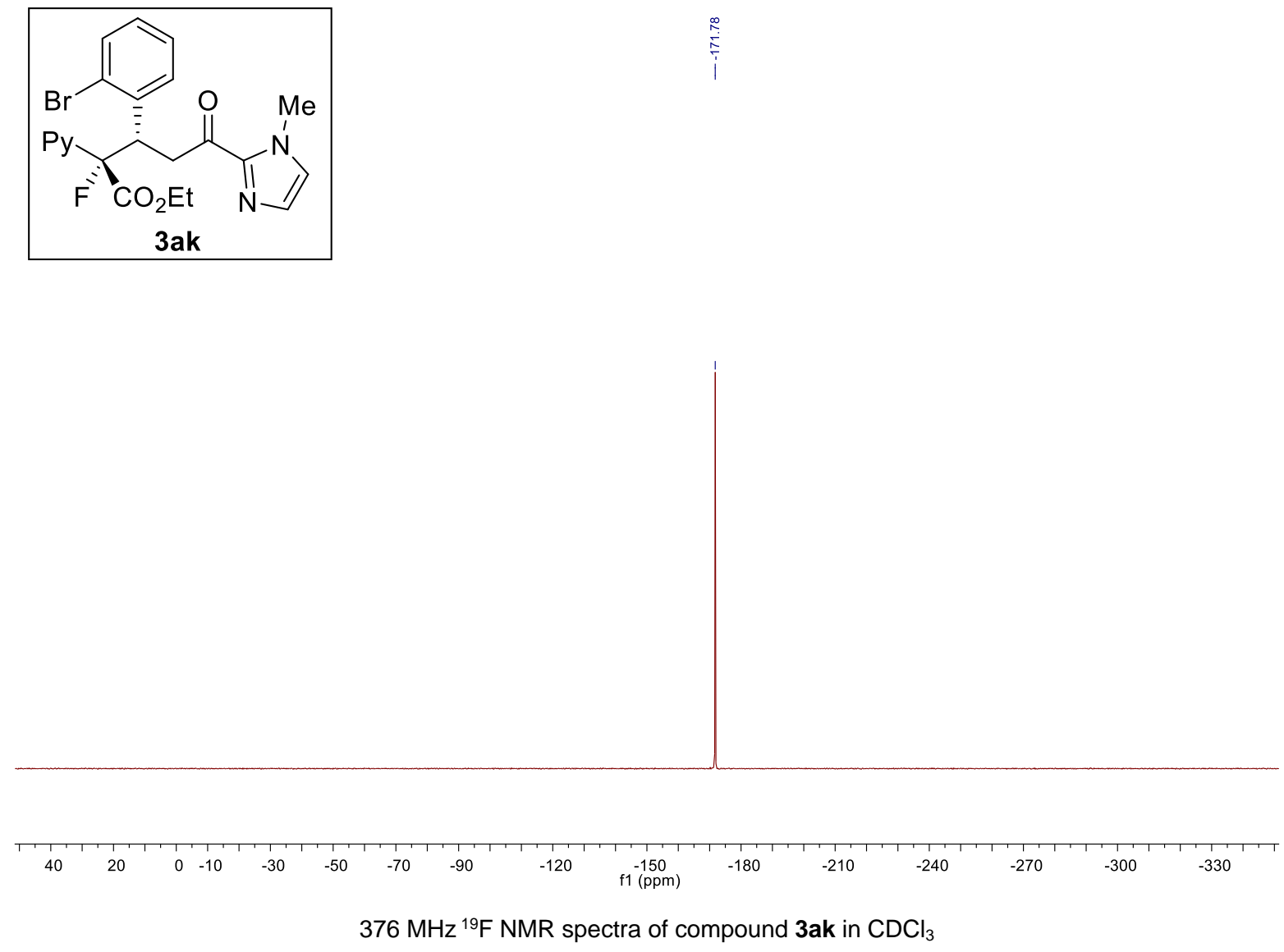

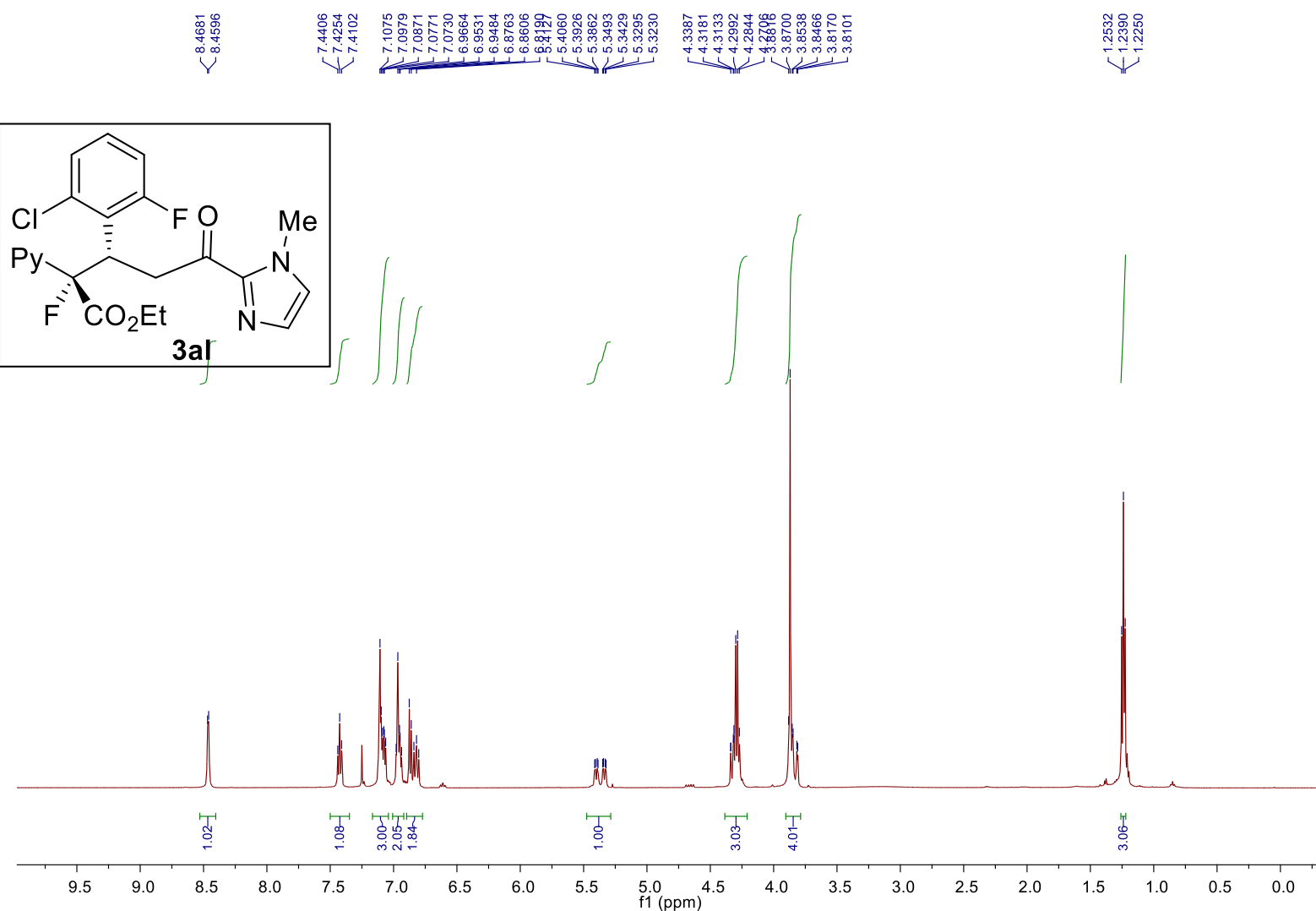

$500 \mathrm{MHz}{ }^{1} \mathrm{H}$ NMR spectra of compound 3al in $\mathrm{CDCl}_{3}$

i
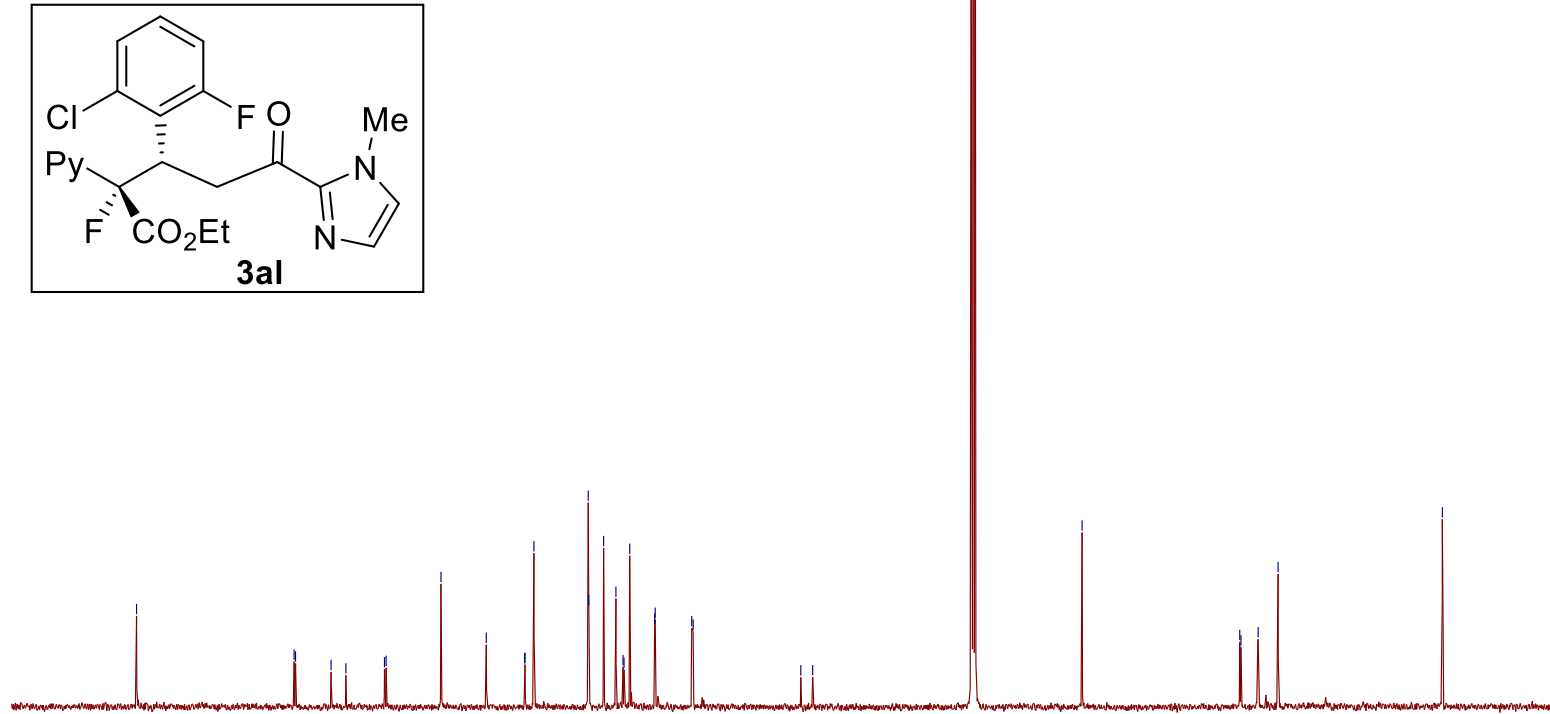

$\begin{array}{llllllllll}200 & 190 & 180 & 170 & 160 & 150 & 140 & 130 & 120 & 110 \begin{array}{l}100 \\ \mathrm{f} 1(\mathrm{ppm})\end{array}\end{array}$

$125 \mathrm{MHz}{ }^{13} \mathrm{C}$ NMR spectra of compound 3al in $\mathrm{CDCl}_{3}$ 


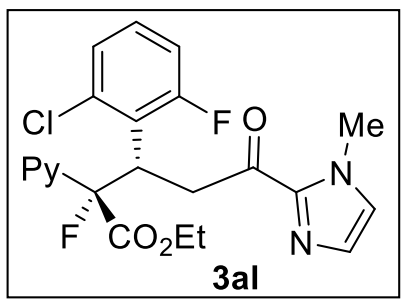

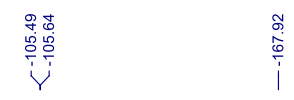

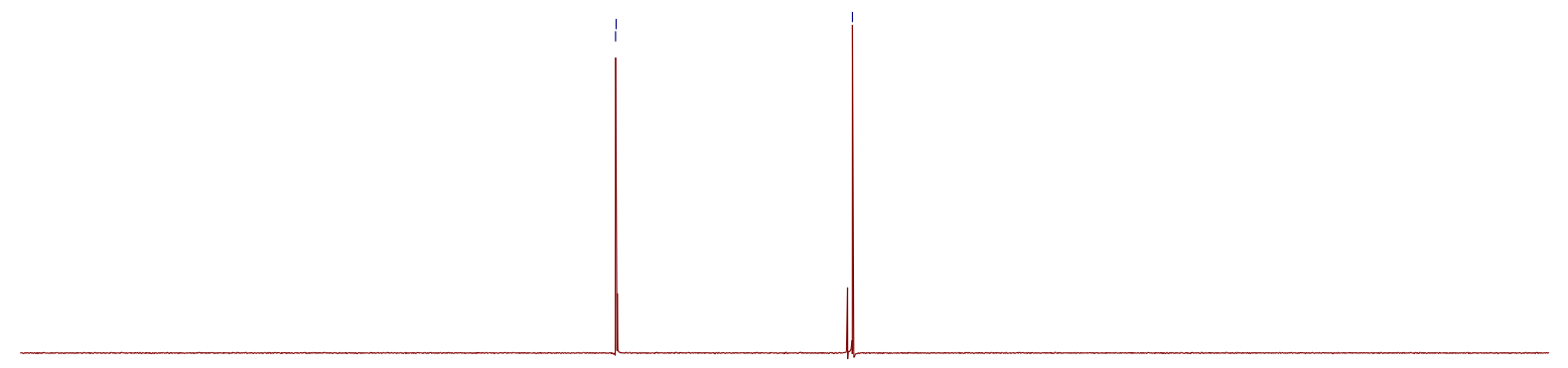

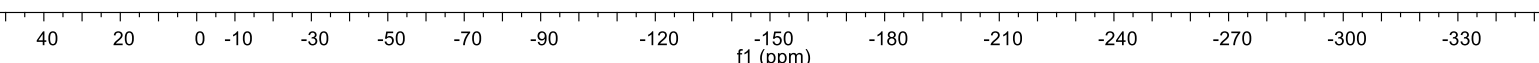

$376 \mathrm{MHz}{ }^{19} \mathrm{~F}$ NMR spectra of compound 3al in $\mathrm{CDCl}_{3}$ 


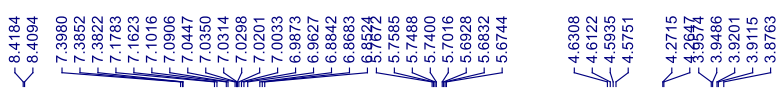
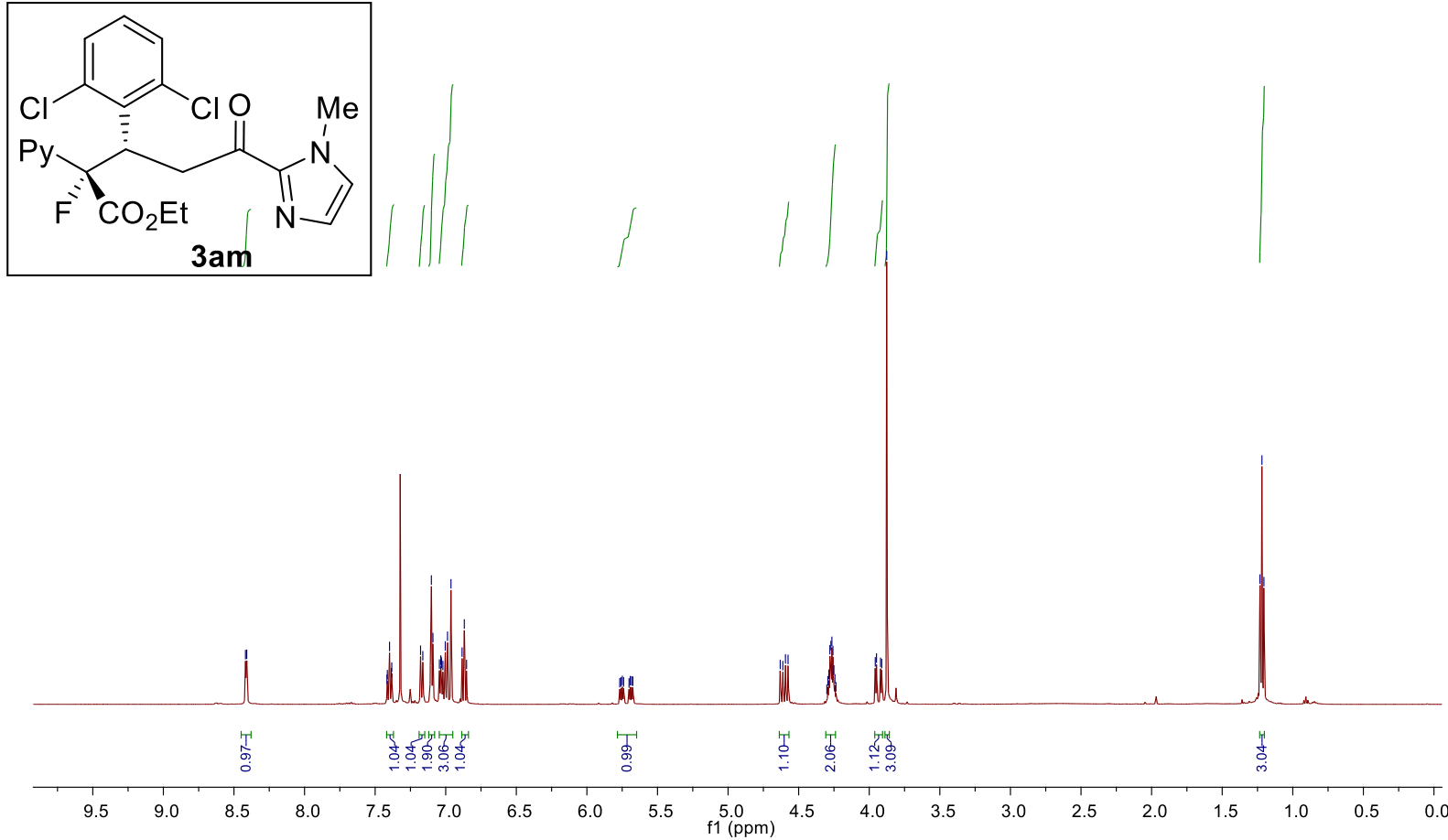

$500 \mathrm{MHz}{ }^{1} \mathrm{H}$ NMR spectra of compound 3am in $\mathrm{CDCl}_{3}$

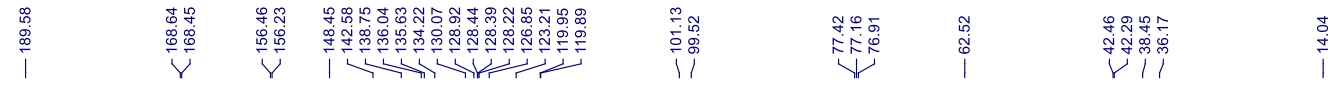
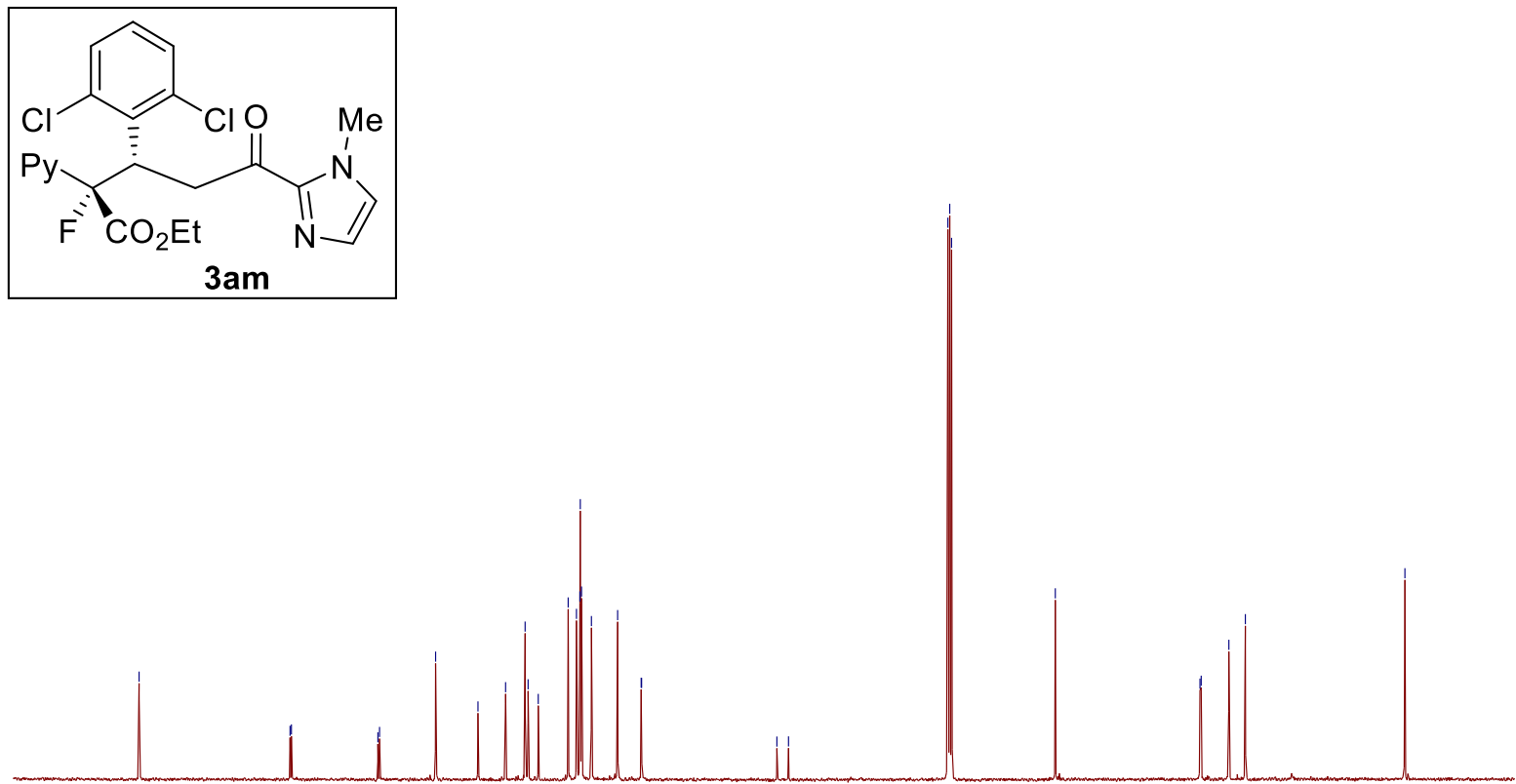

$\begin{array}{lllllllllllllllllllll}200 & 190 & 180 & 170 & 160 & 150 & 140 & 130 & 120 & \begin{array}{c}110 \\ \mathrm{f} 1(\mathrm{ppm})\end{array} & 90 & 80 & 70 & 60 & 50 & 40 & 30 & 20 & 10 & 0\end{array}$ $125 \mathrm{MHz}{ }^{13} \mathrm{C}$ NMR spectra of compound 3am in $\mathrm{CDCl}_{3}$ 

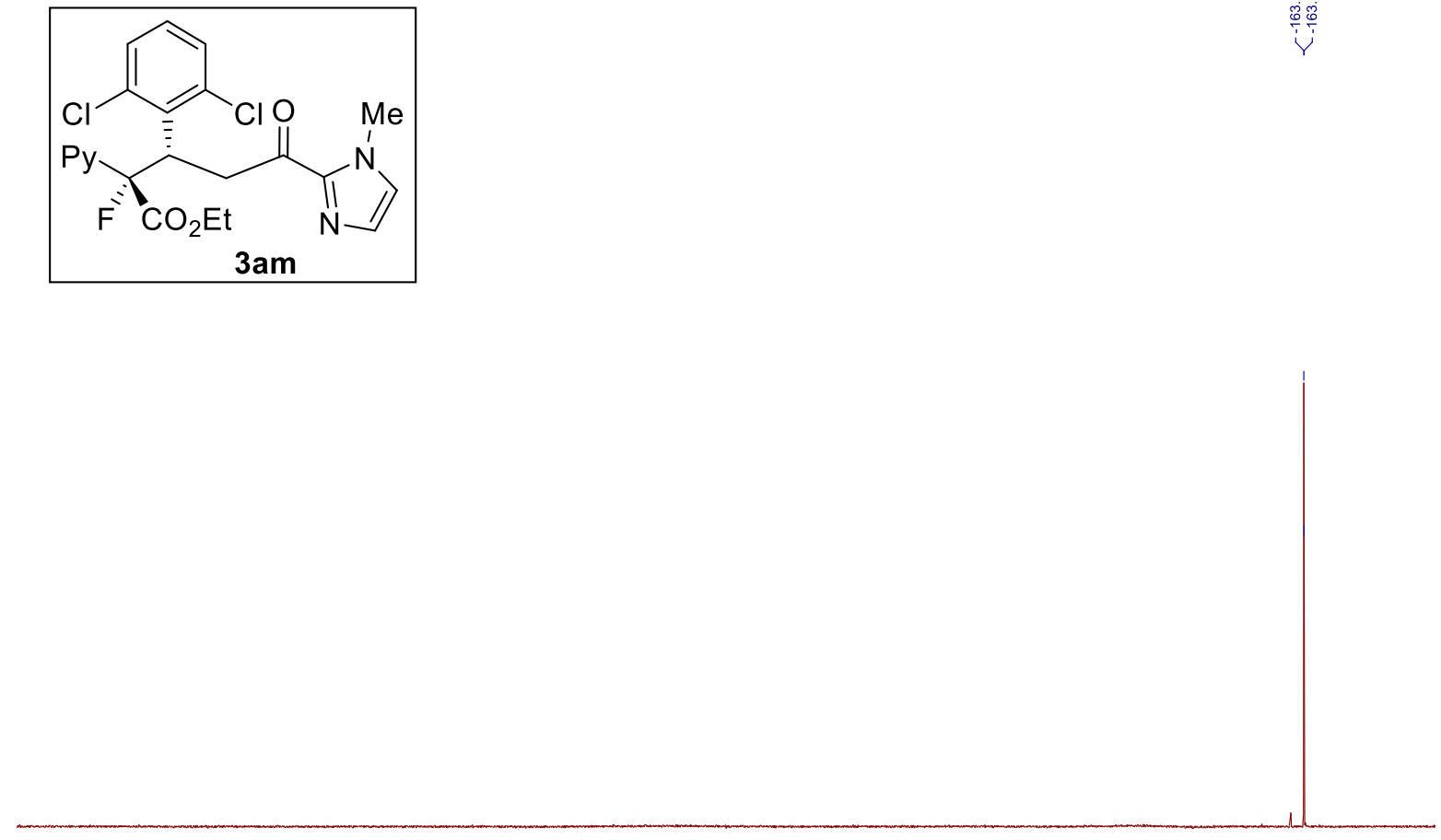

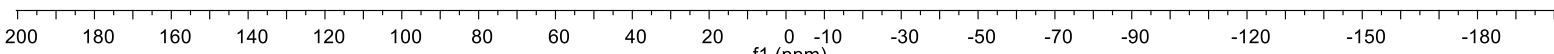
$376 \mathrm{MHz}{ }^{19} \mathrm{~F}$ NMR spectra of compound 3am in $\mathrm{CDCl}_{3}$ 

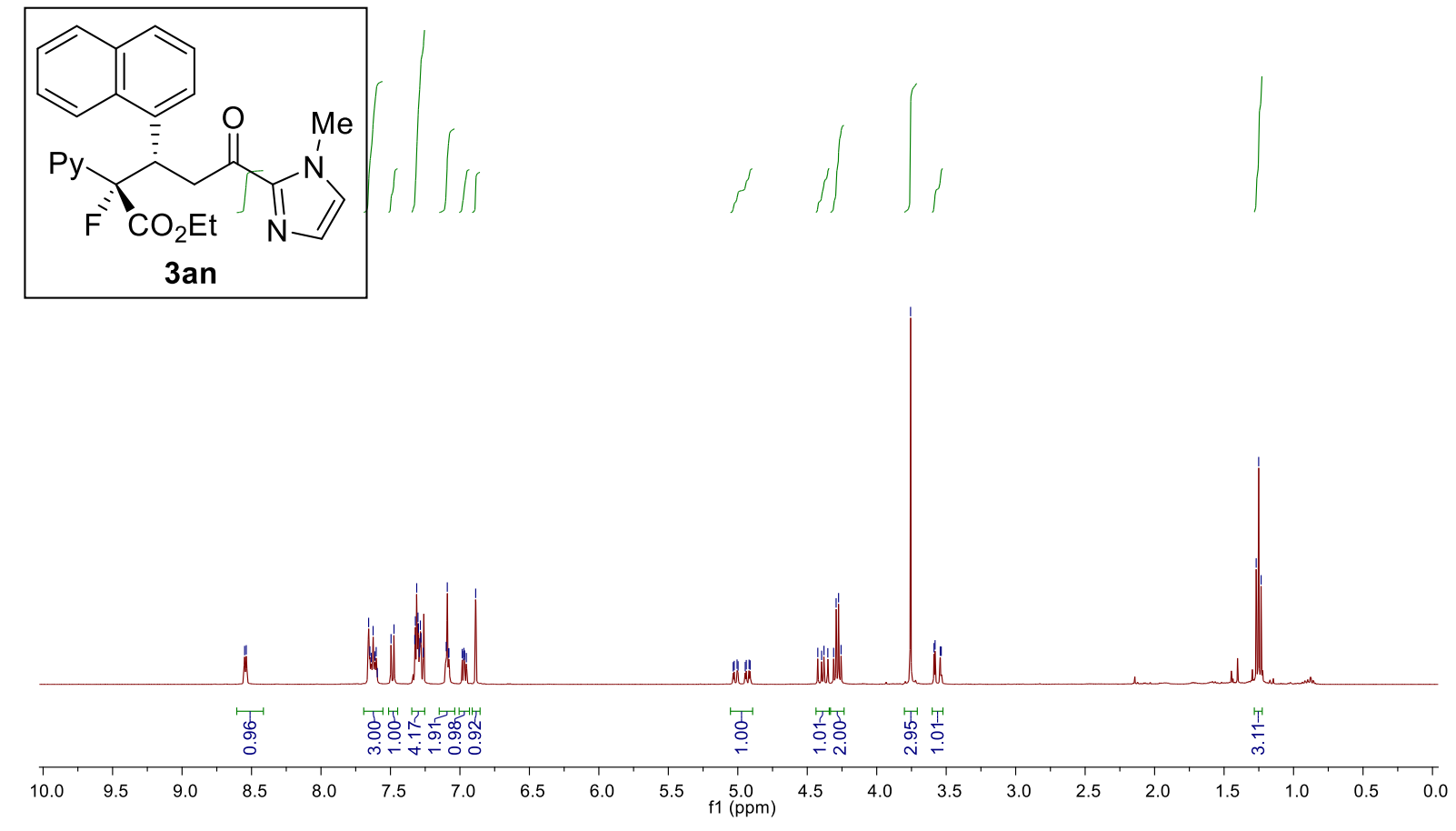

$400 \mathrm{MHz}{ }^{1} \mathrm{H}$ NMR spectra of compound 3an in $\mathrm{CDCl}_{3}$

V V

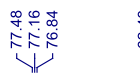

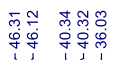
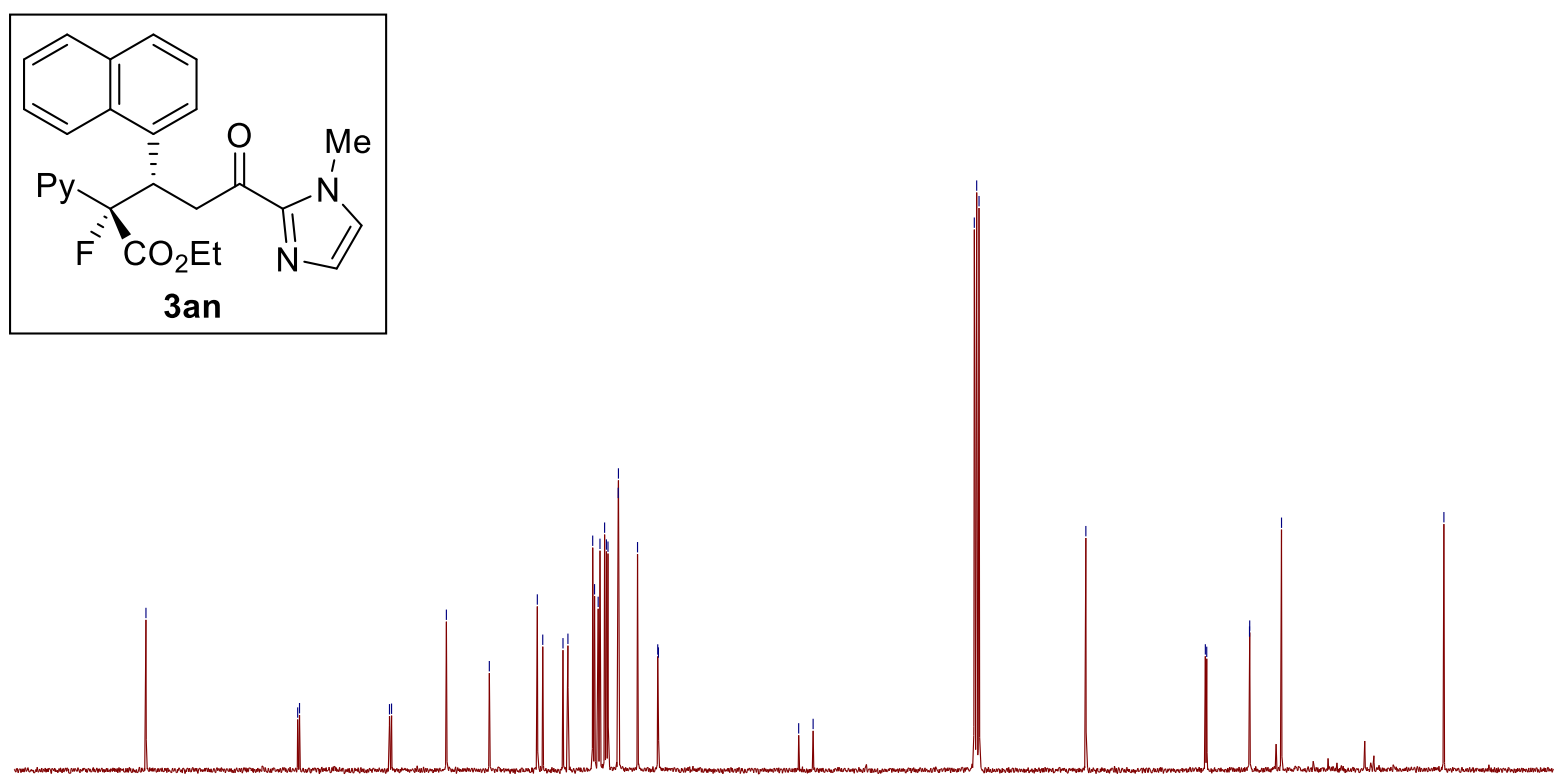

$\begin{array}{llllllllll}200 & 190 & 180 & 170 & 160 & 150 & 140 & 130 & 120 & 110 \\ \mathrm{f} 1(\mathrm{ppm}) & 100\end{array}$

$100 \mathrm{MHz}{ }^{13} \mathrm{C}$ NMR spectra of compound 3an in $\mathrm{CDCl}_{3}$ 

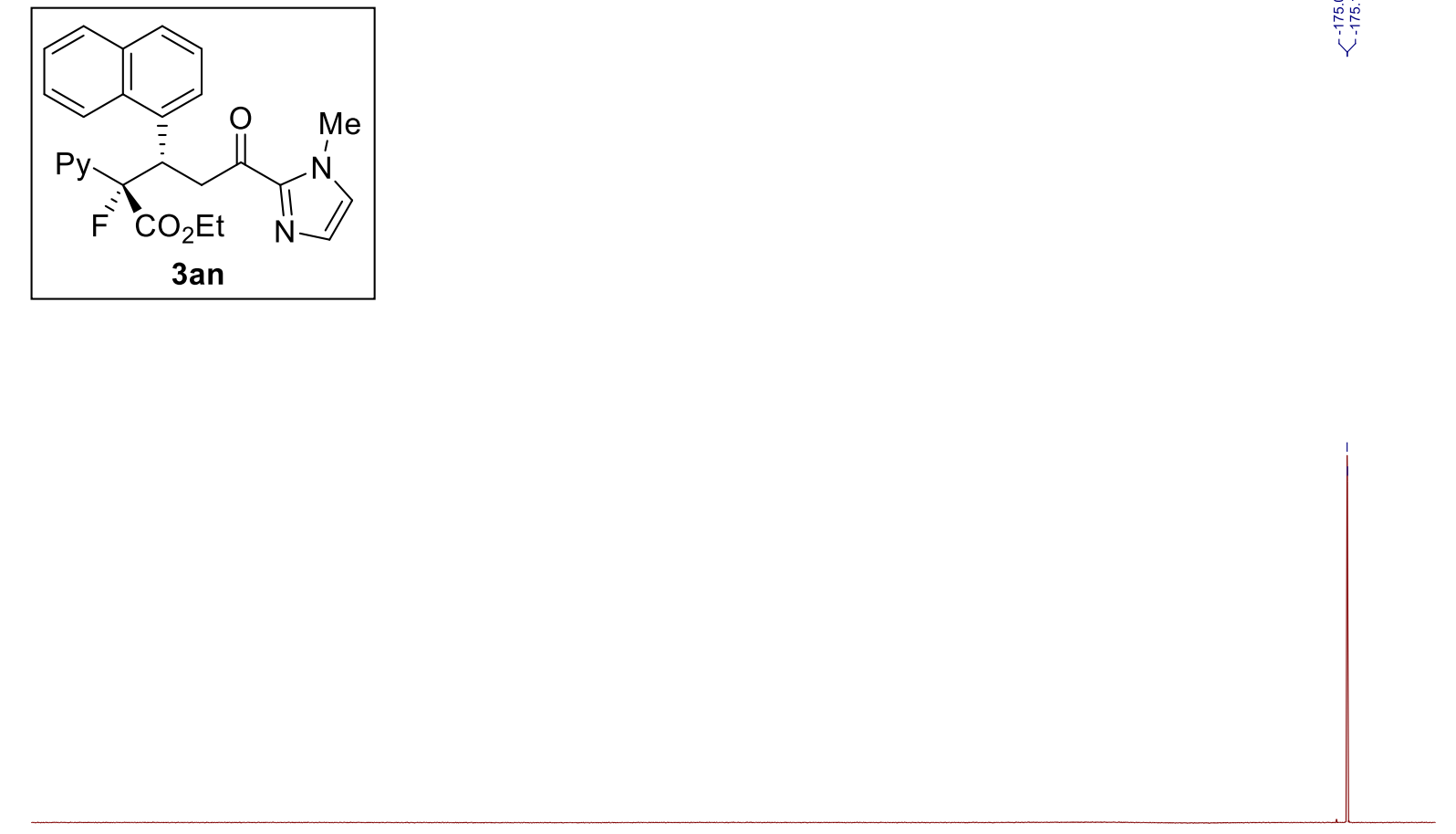

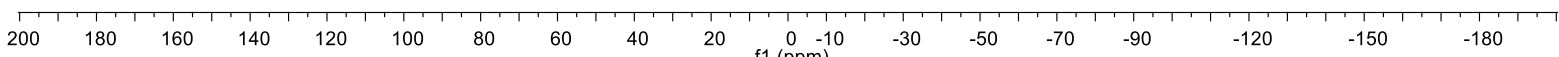

$376 \mathrm{MHz}{ }^{19} \mathrm{~F}$ NMR spectra of compound 3an in $\mathrm{CDCl}_{3}$ 


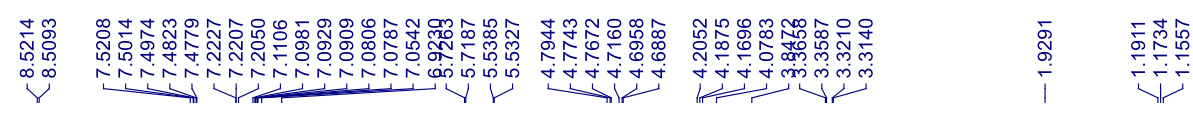
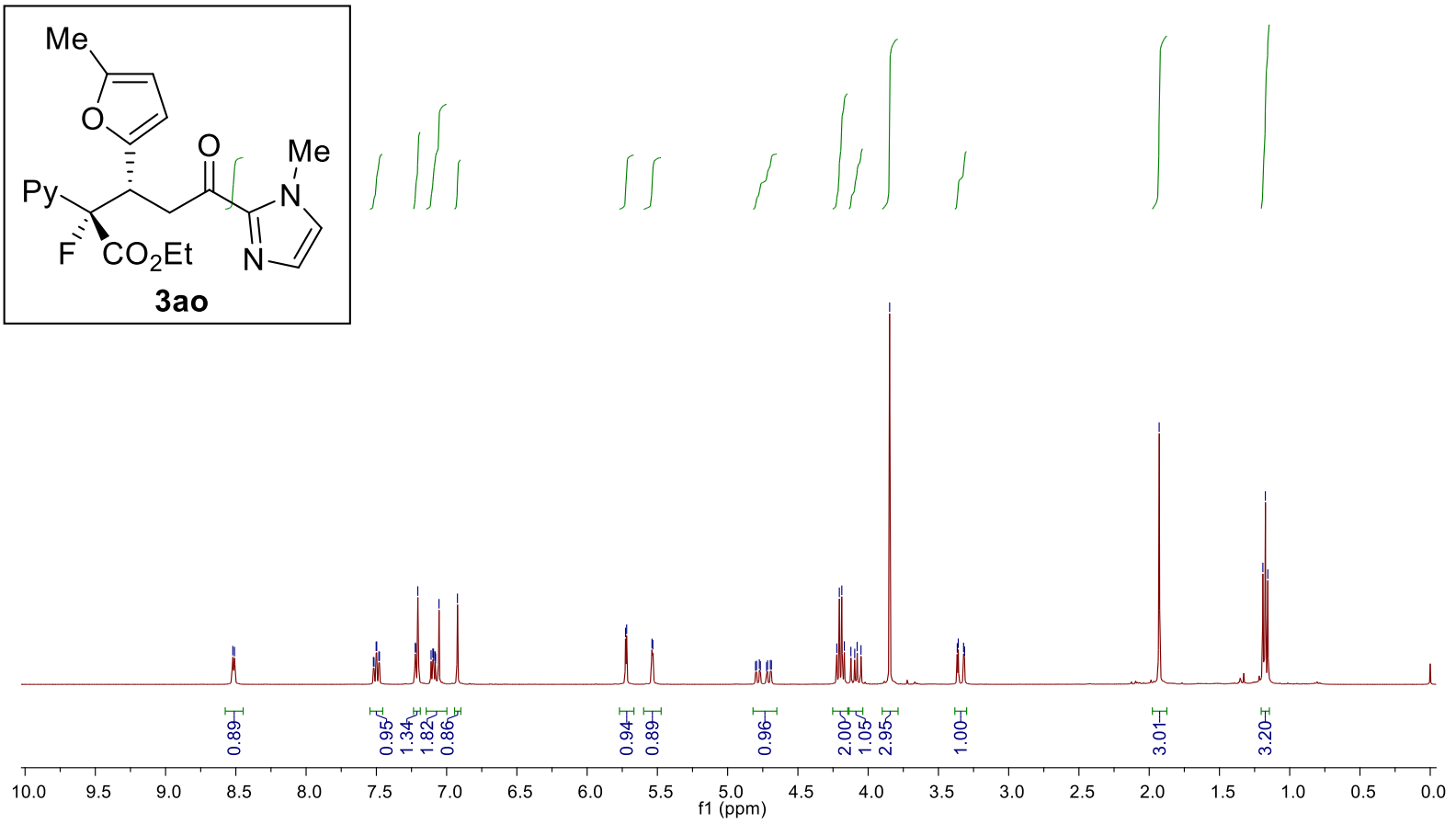

$400 \mathrm{MHz}{ }^{1} \mathrm{H}$ NMR spectra of compound $3 \mathrm{ao}$ in $\mathrm{CDCl}_{3}$

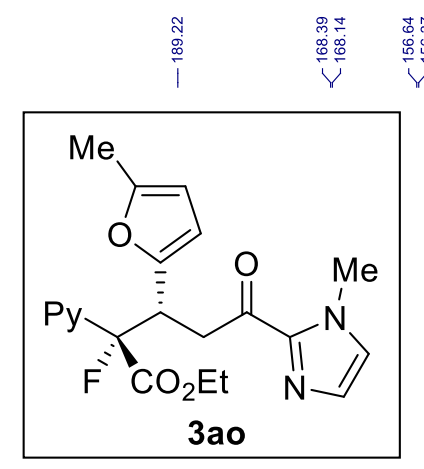

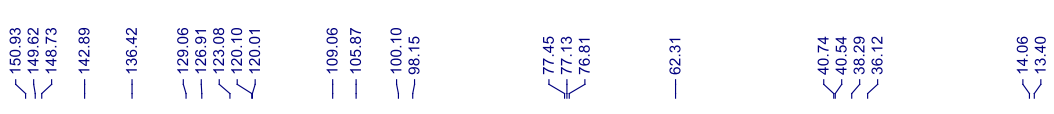



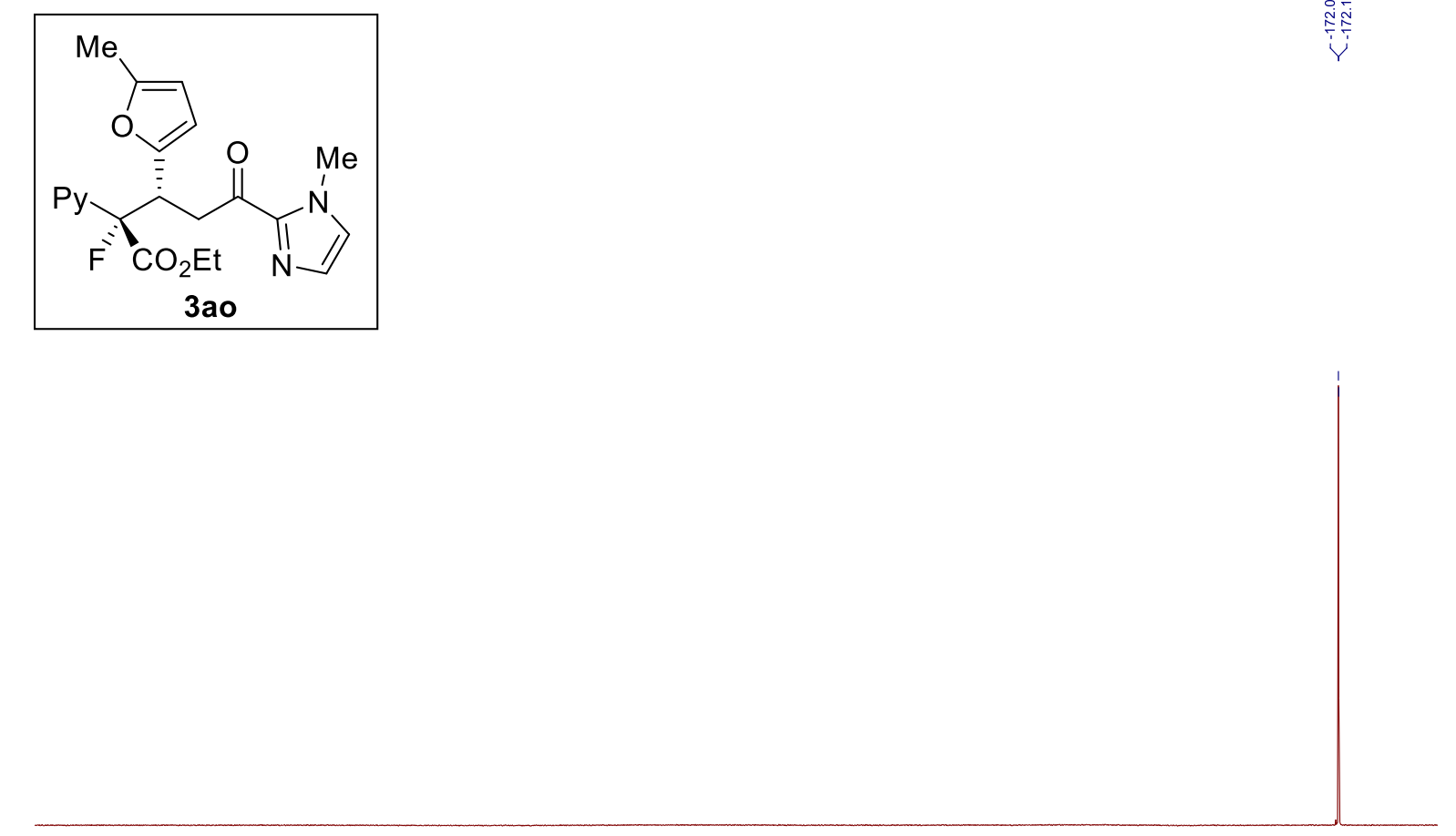

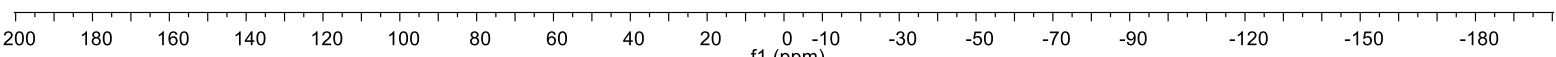
$376 \mathrm{MHz}{ }^{19} \mathrm{~F}$ NMR spectra of compound $3 \mathrm{ao}$ in $\mathrm{CDCl}_{3}$ 

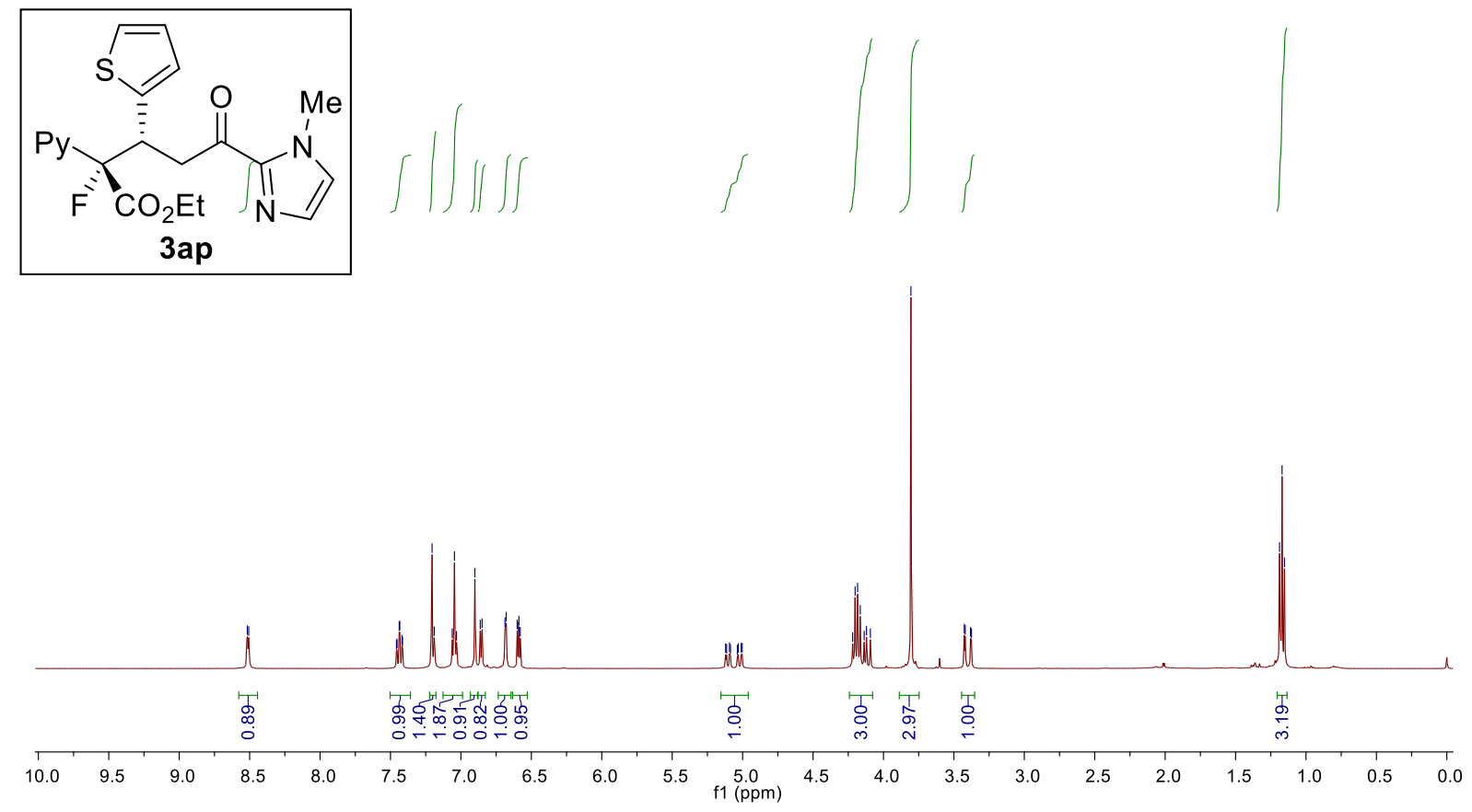

$400 \mathrm{MHz}{ }^{1} \mathrm{H}$ NMR spectra of compound 3ap in $\mathrm{CDCl}_{3}$

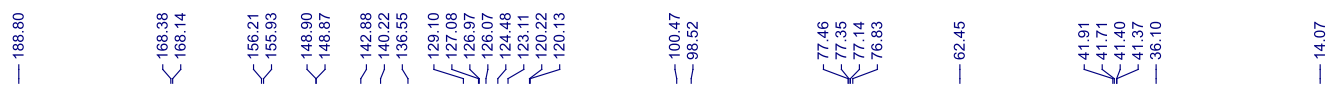
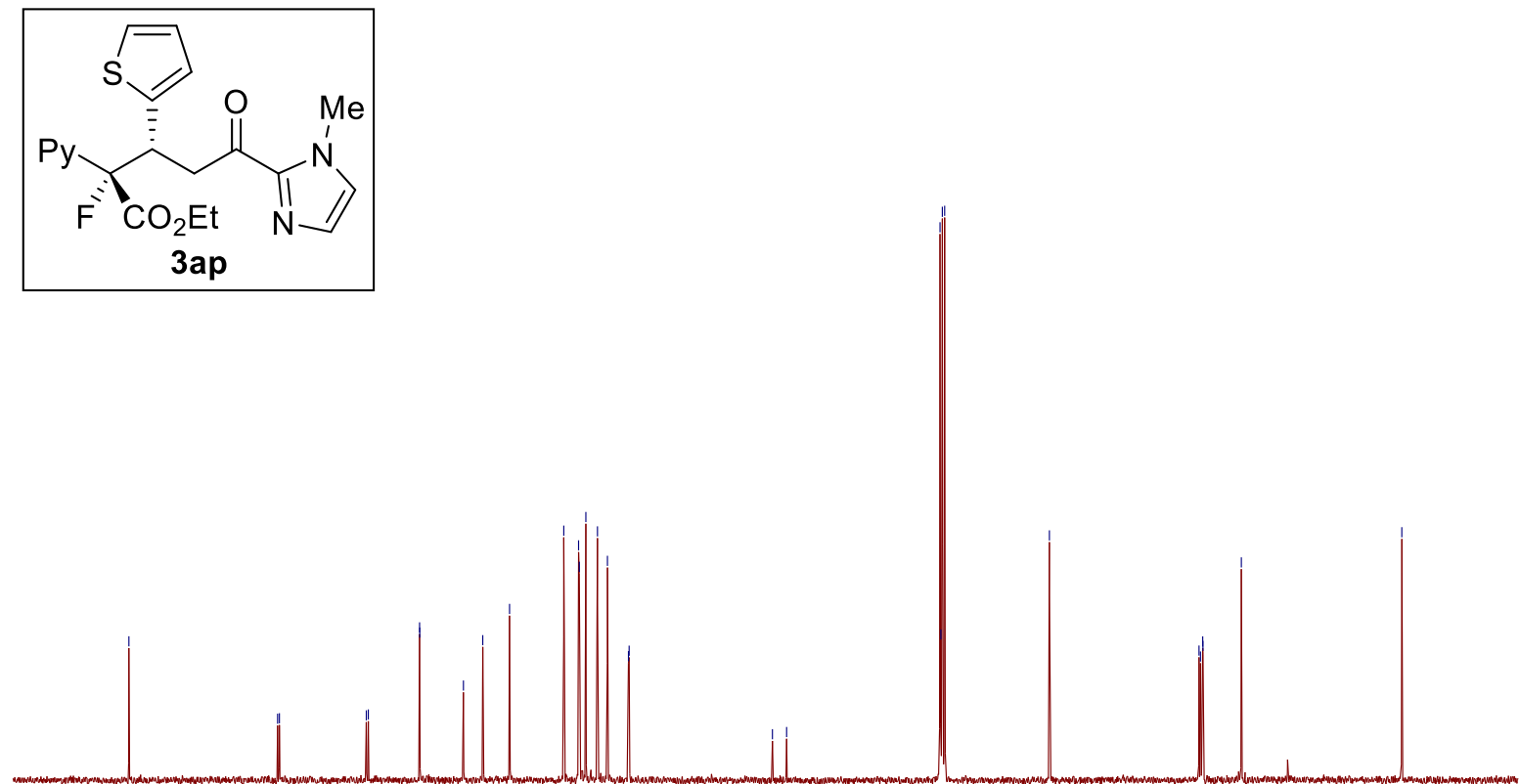

$100 \mathrm{MHz}{ }^{13} \mathrm{C}$ NMR spectra of compound 3ap in $\mathrm{CDCl}_{3}$ 

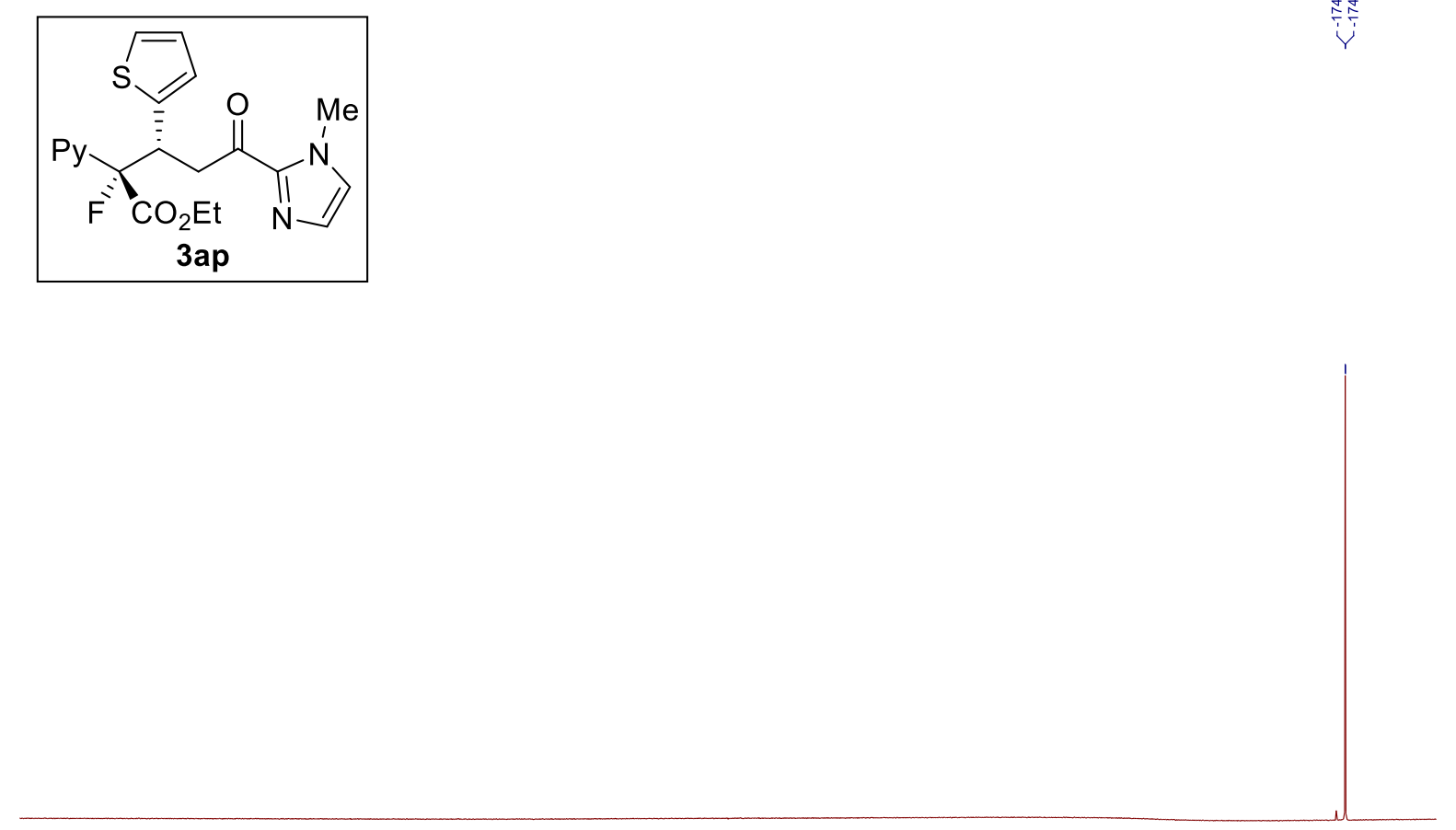

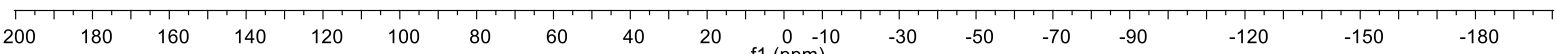
$376 \mathrm{MHz}{ }^{19} \mathrm{~F}$ NMR spectra of compound 3ap in $\mathrm{CDCl}_{3}$ 


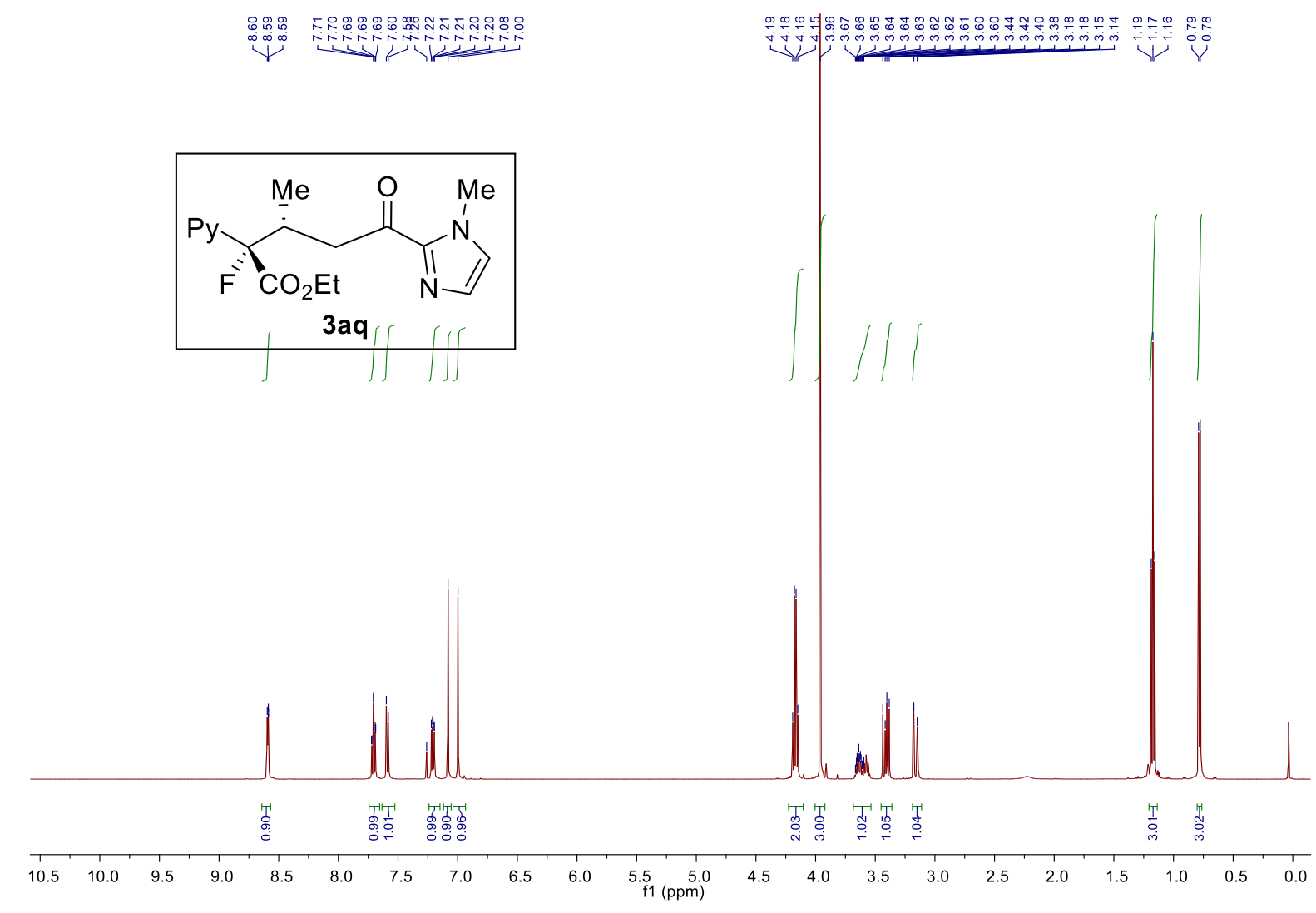

$400 \mathrm{MHz}{ }^{1} \mathrm{H}$ NMR spectra of compound 3aq in $\mathrm{CDCl}_{3}$

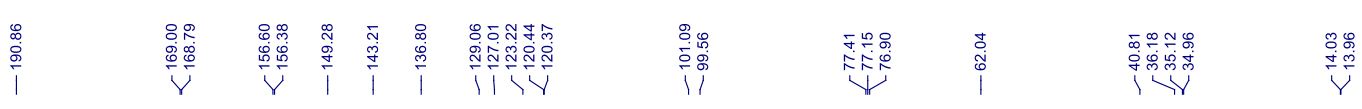

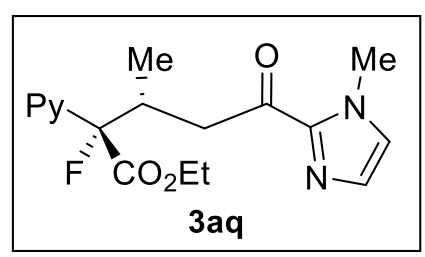

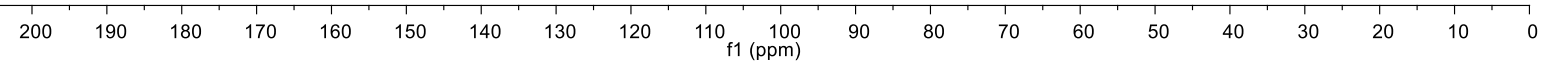

$125 \mathrm{MHz}{ }^{13} \mathrm{C}$ NMR spectra of compound 3aq in $\mathrm{CDCl}_{3}$ 


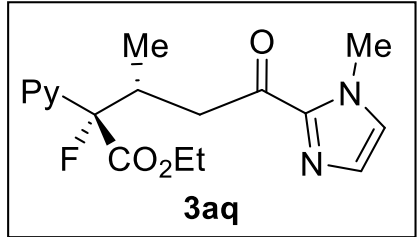

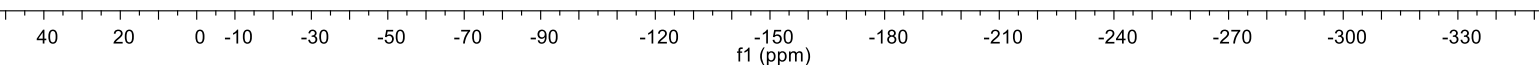

$376 \mathrm{MHz}{ }^{19} \mathrm{~F}$ NMR spectra of compound $3 \mathrm{aq}$ in $\mathrm{CDCl}_{3}$ 


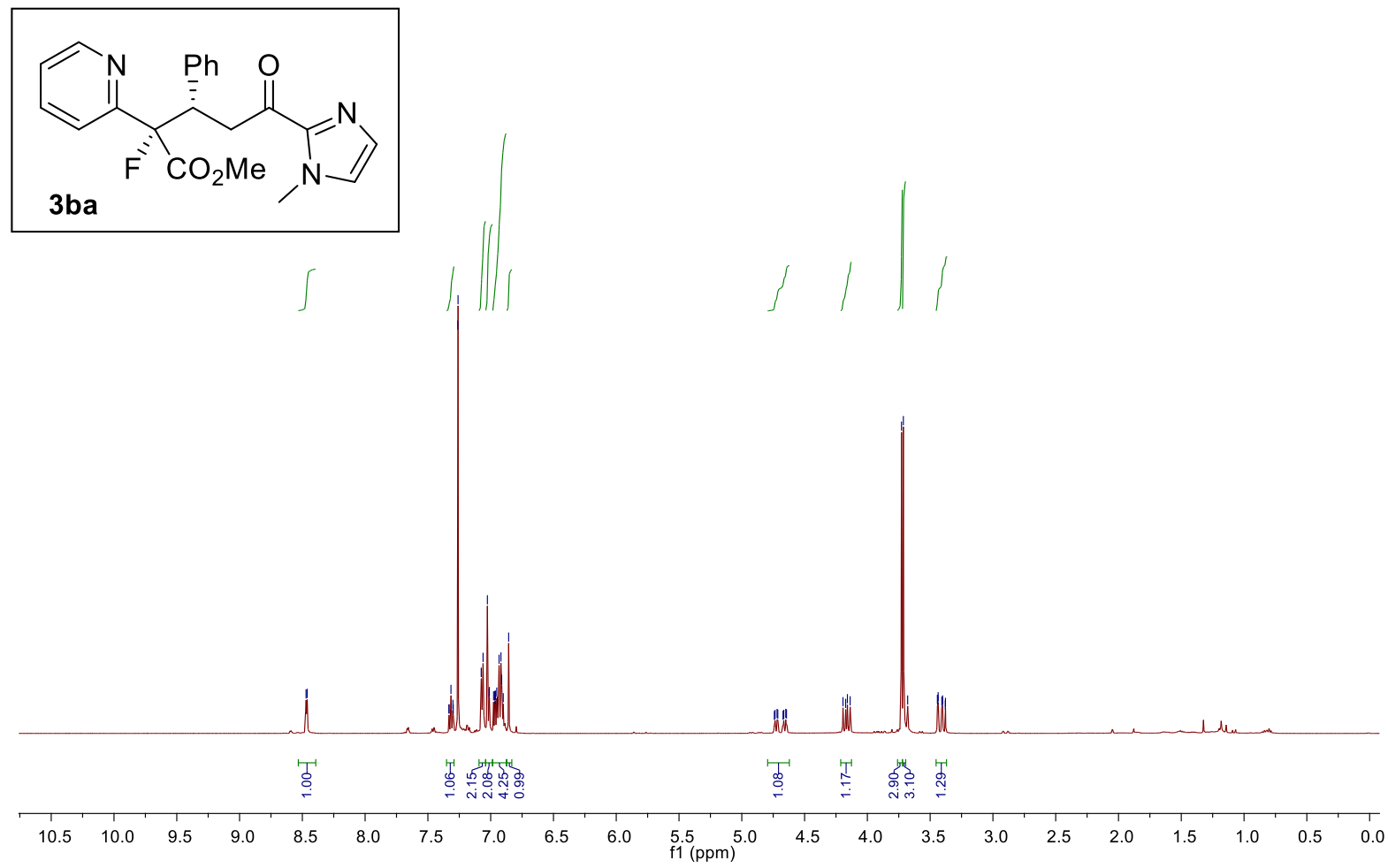

$500 \mathrm{MHz}{ }^{1} \mathrm{H}$ NMR spectra of compound 3 ba in $\mathrm{CDCl}_{3}$

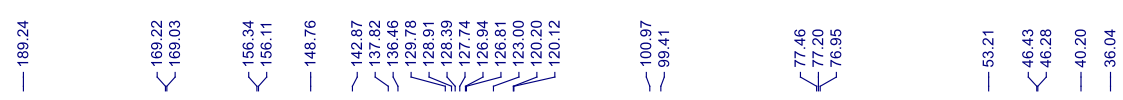
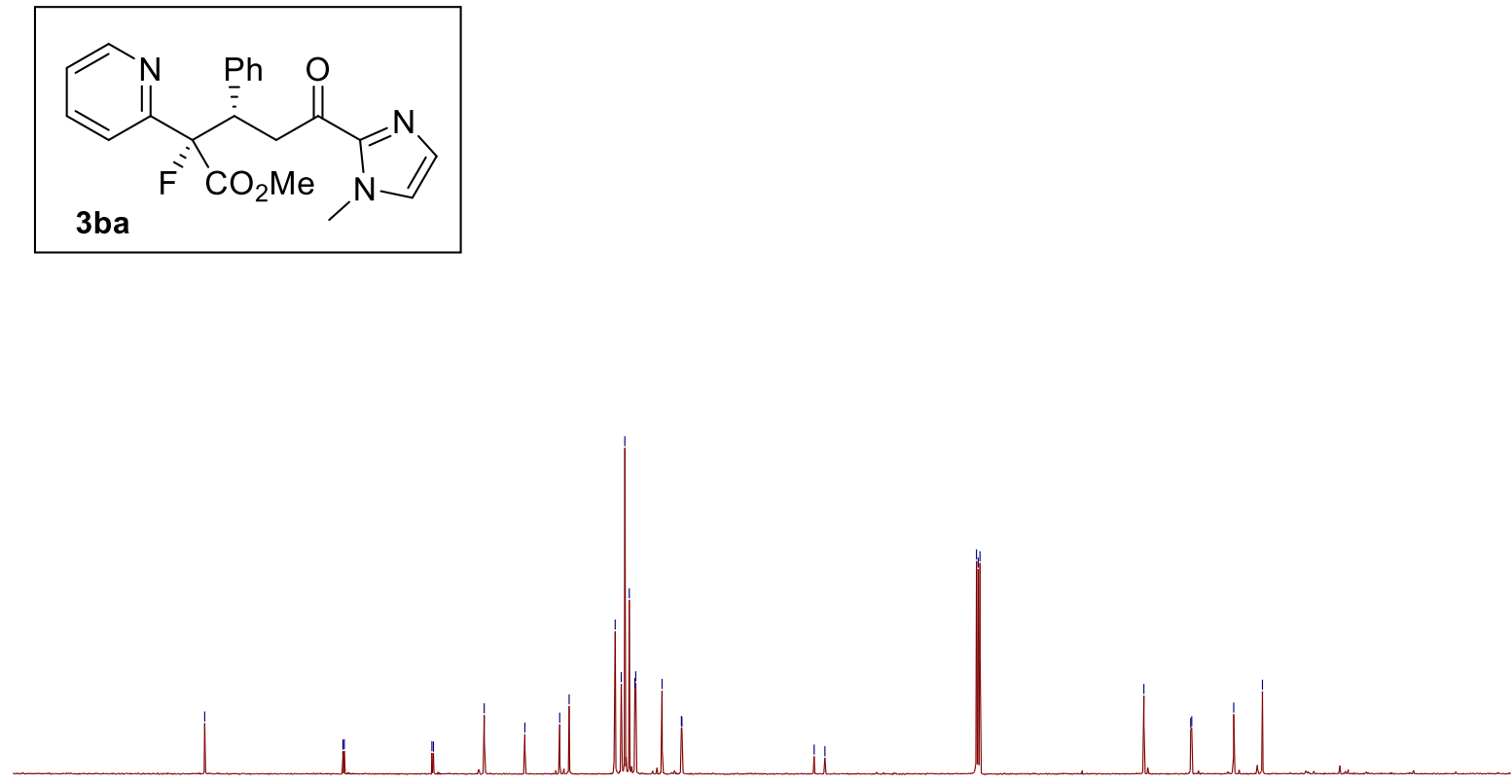

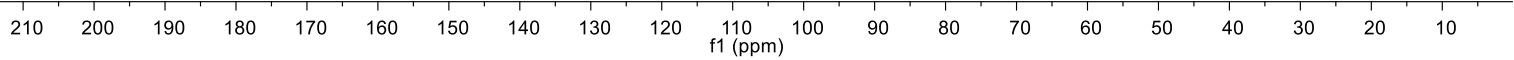
$125 \mathrm{MHz}{ }^{13} \mathrm{C}$ NMR spectra of compound 3 ba in $\mathrm{CDCl}_{3}$ 


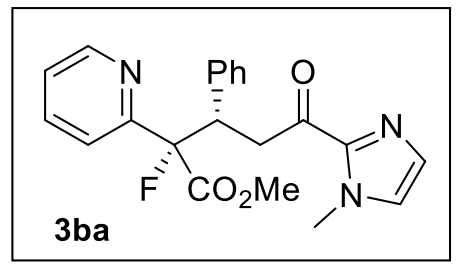

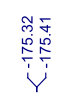

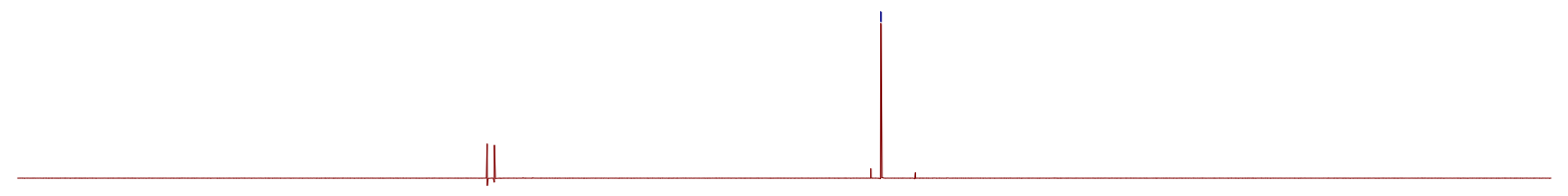

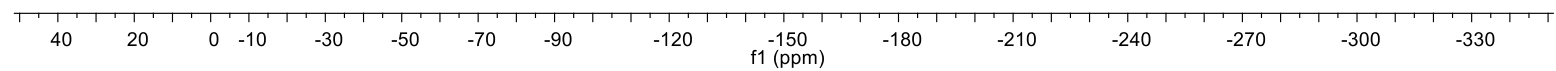

$376 \mathrm{MHz}{ }^{19} \mathrm{~F}$ NMR spectra of compound 3 ba in $\mathrm{CDCl}_{3}$ 


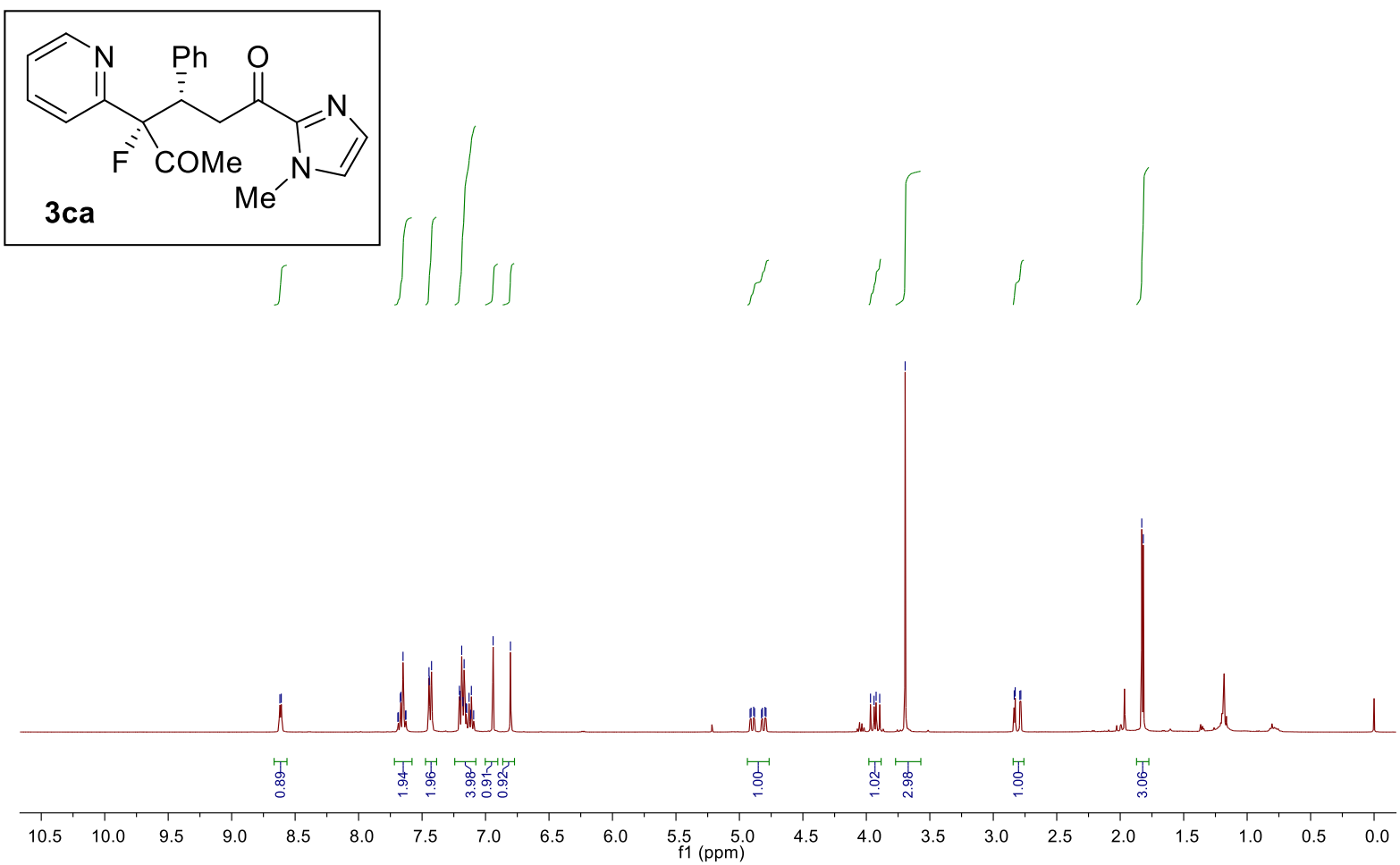

$400 \mathrm{MHz}{ }^{1} \mathrm{H}$ NMR spectra of compound 3 ba in $\mathrm{CDCl}_{3}$

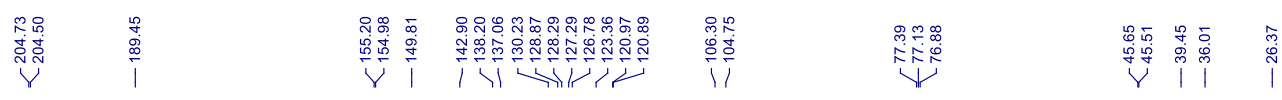
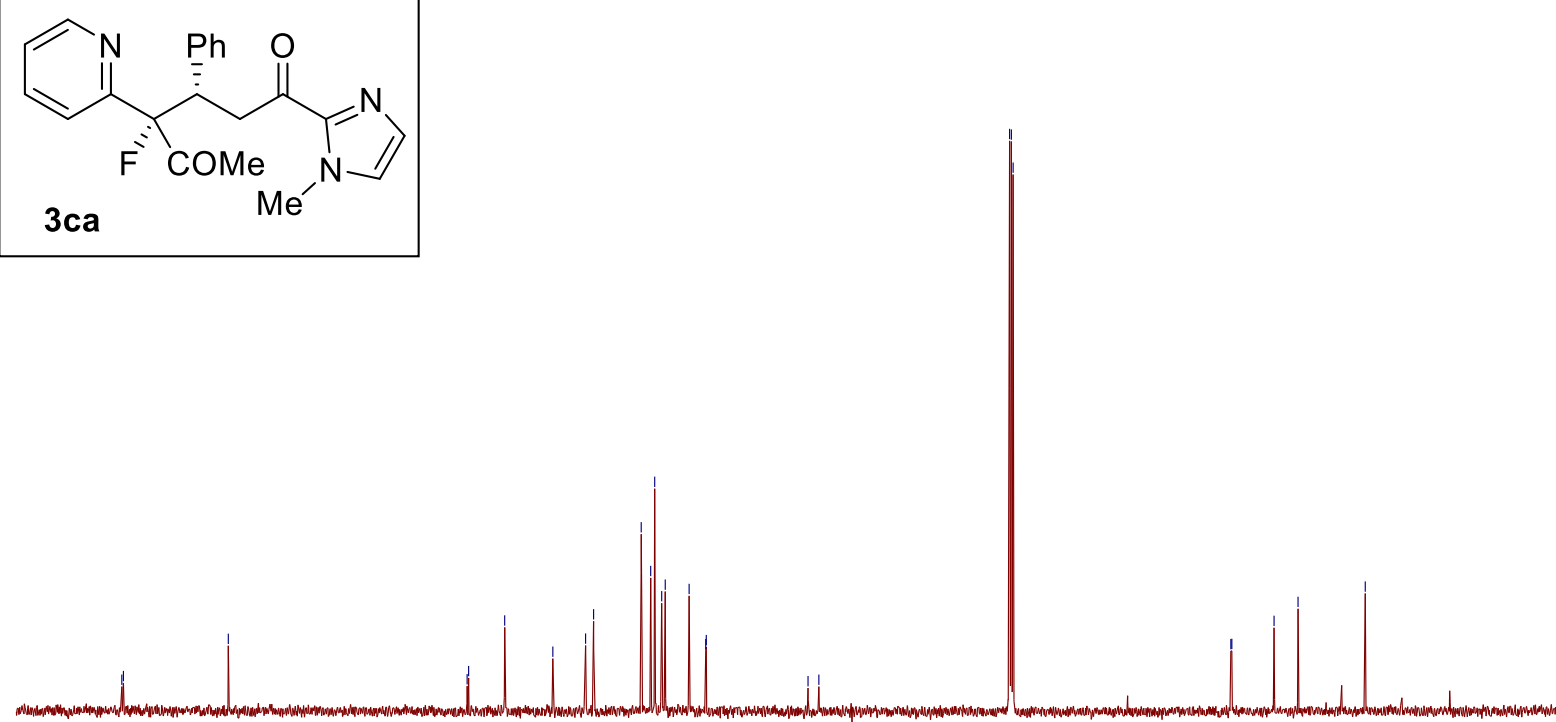

$\begin{array}{llllllllllllllllllllllllll} & 210 & 200 & 190 & 180 & 170 & 160 & 150 & 140 & 130 & 120 & 110 & 100 & 90 & 80 & 70 & 60 & 50 & 40 & 30 & 20 & 10 & 0\end{array}$

$125 \mathrm{MHz}{ }^{13} \mathrm{C}$ NMR spectra of compound 3 ba in $\mathrm{CDCl}_{3}$ 

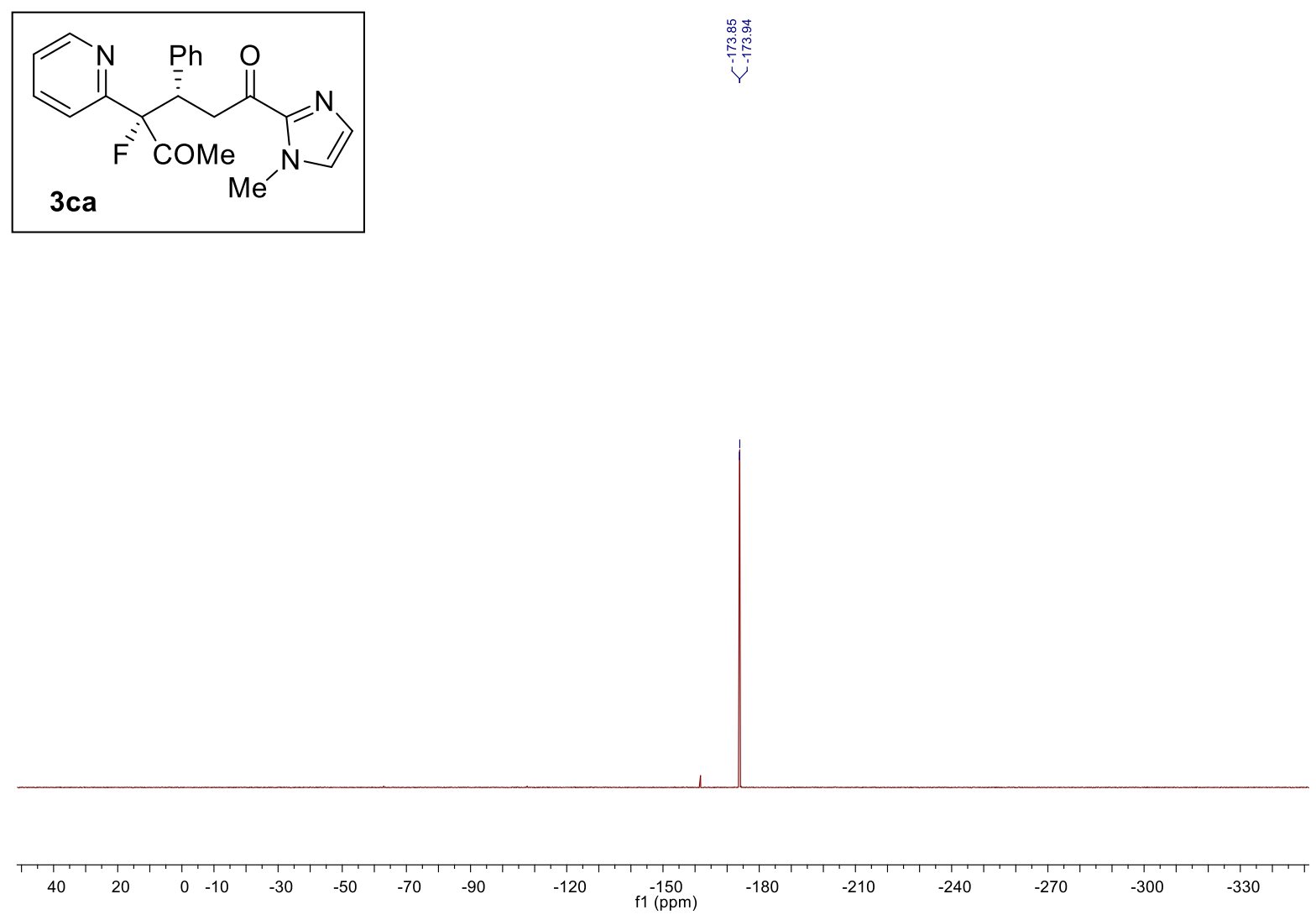

$376 \mathrm{MHz}{ }^{19} \mathrm{~F}$ NMR spectra of compound 3ca in $\mathrm{CDCl}_{3}$ 

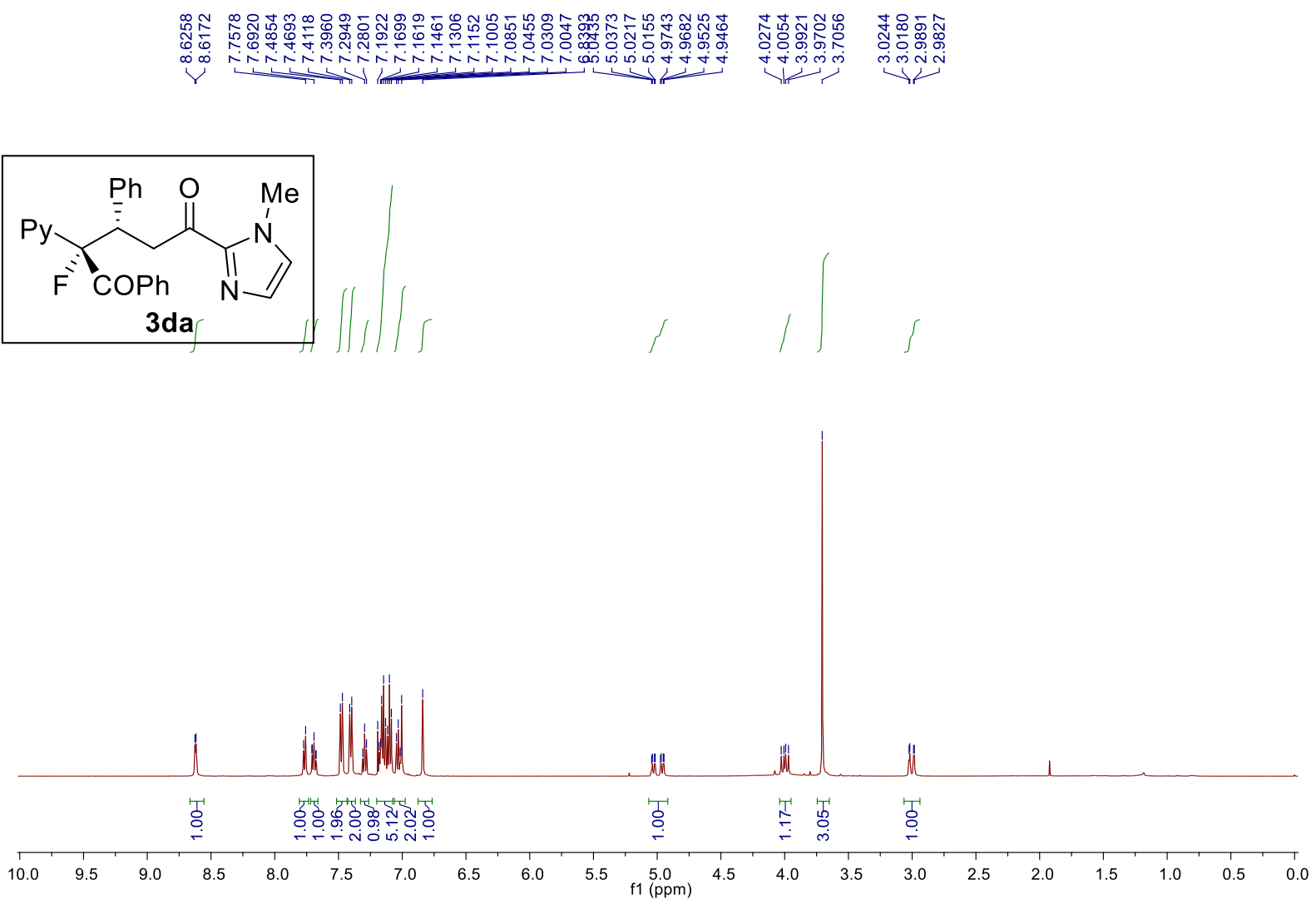
$500 \mathrm{MHz}{ }^{1} \mathrm{H}$ NMR spectra of compound 3da in $\mathrm{CDCl}_{3}$

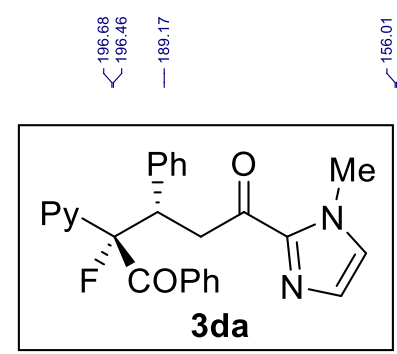

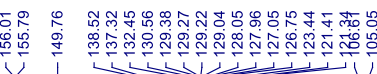

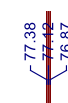

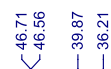

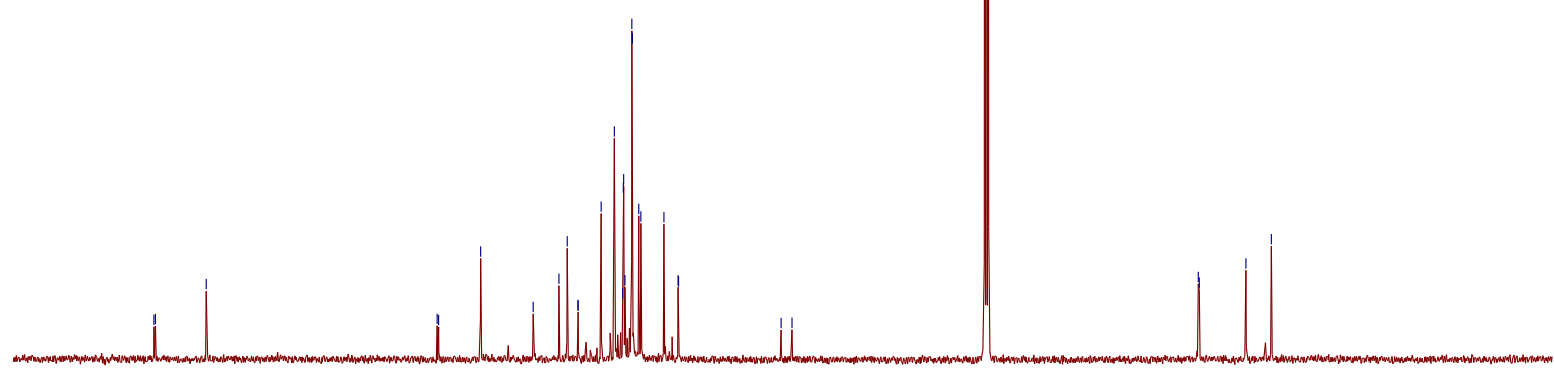

$125 \mathrm{MHz}{ }^{13} \mathrm{C}$ NMR spectra of compound 3da in $\mathrm{CDCl}_{3}$ 

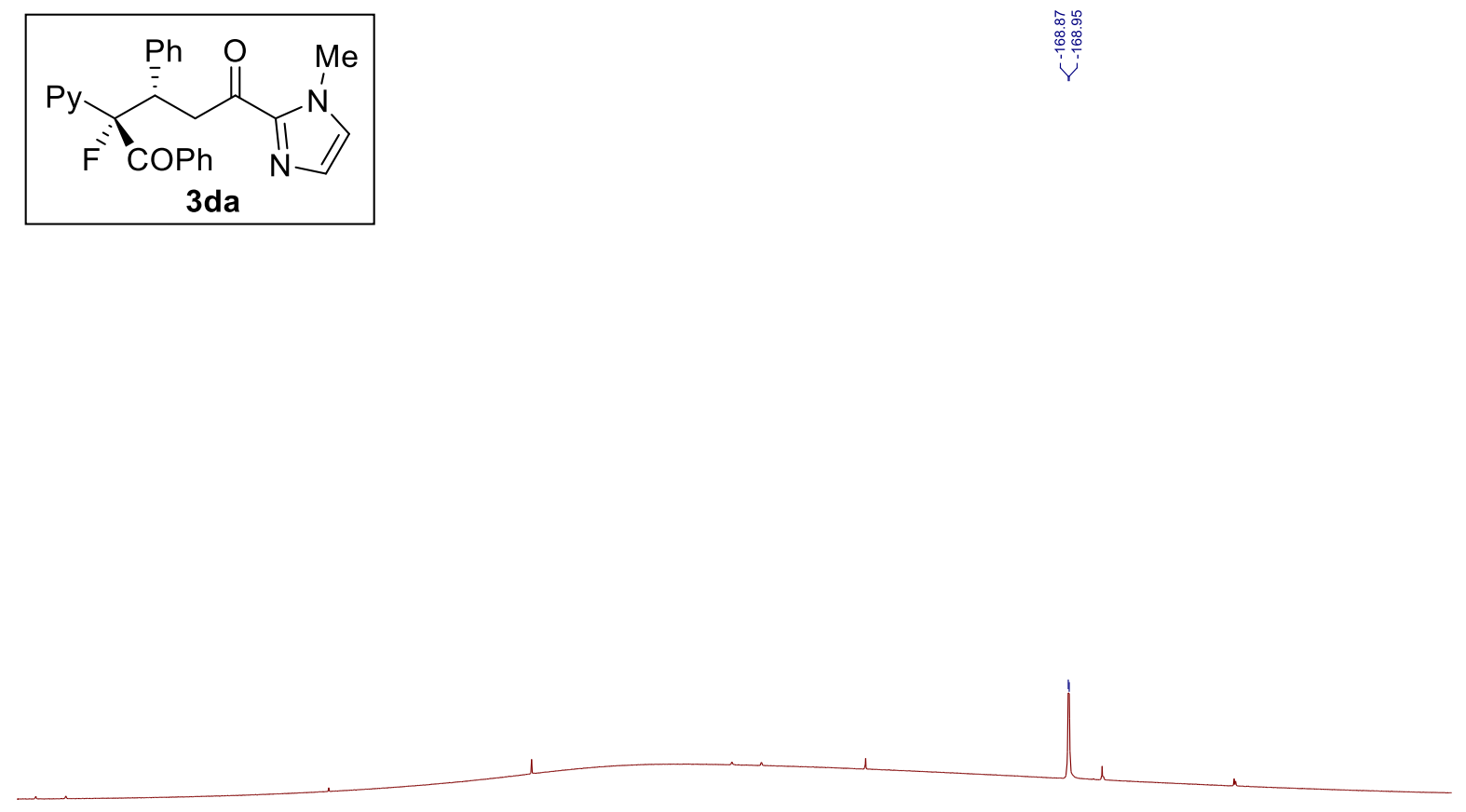

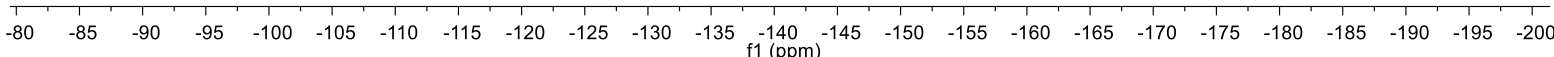
$376 \mathrm{MHz}{ }^{19} \mathrm{~F}$ NMR spectra of compound $3 \mathrm{da}$ in $\mathrm{CDCl}_{3}$ 

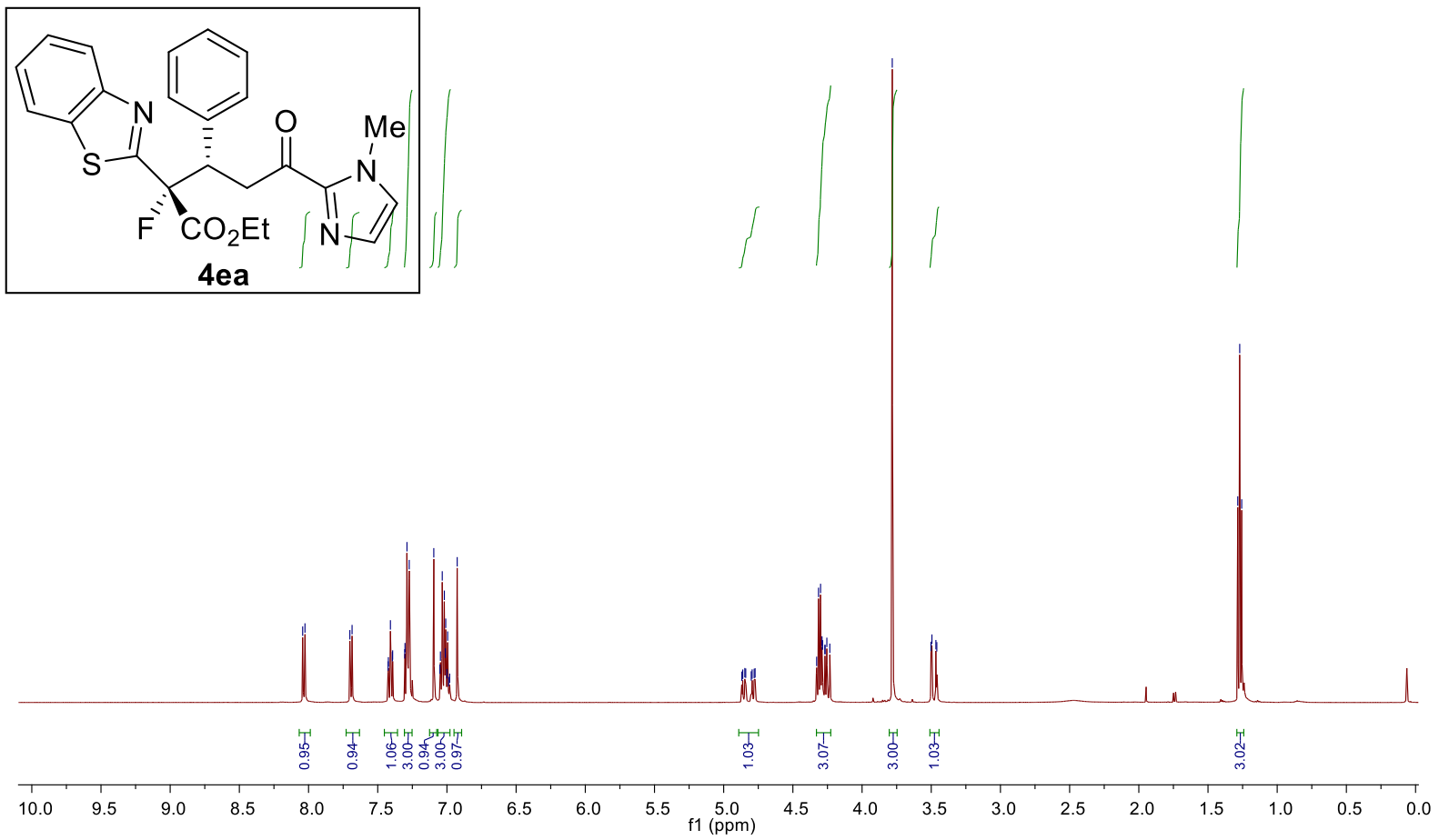

$500 \mathrm{MHz}{ }^{1} \mathrm{H}$ NMR spectra of compound 4ea in $\mathrm{CDCl}_{3}$

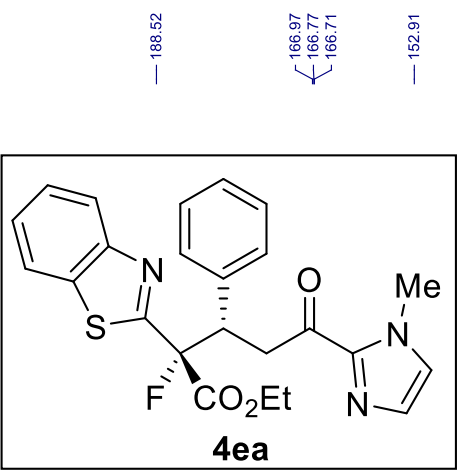

|

80
in
0
0
11

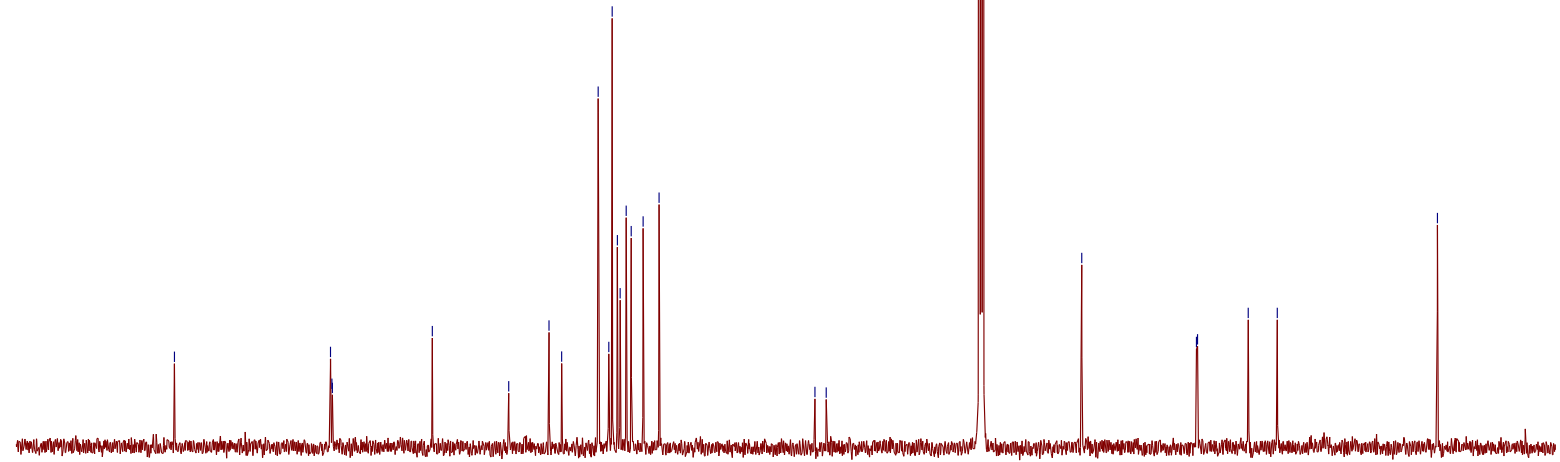

$\begin{array}{lllllllllll}210 & 200 & 190 & 180 & 170 & 160 & 150 & 140 & 130 & 120 & 110 \\ \mathrm{f} 1(\mathrm{ppm}) & 100\end{array}$

$125 \mathrm{MHz}{ }^{13} \mathrm{C}$ NMR spectra of compound 4ea in $\mathrm{CDCl}_{3}$ 

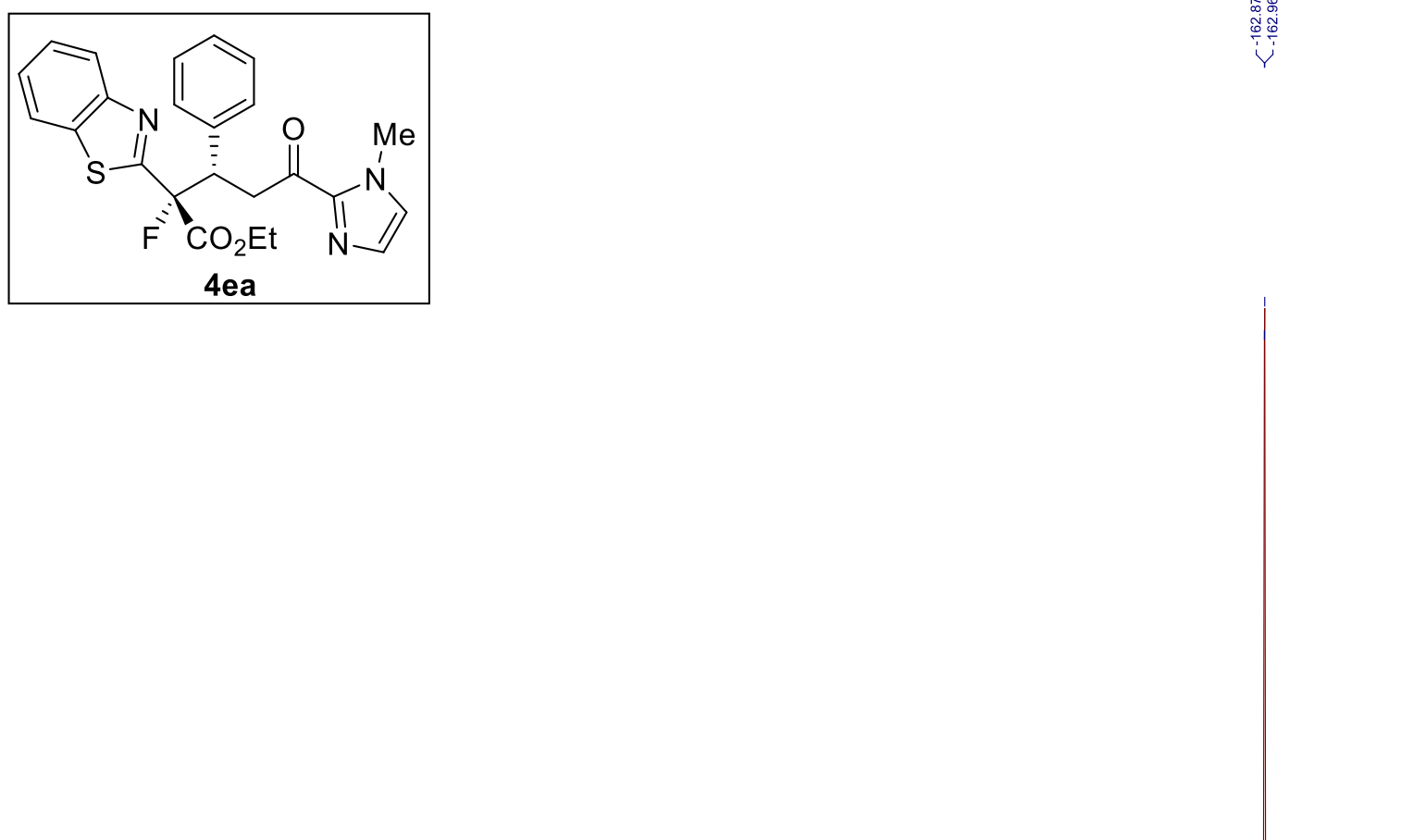

$200 \quad 180 \quad 160 \quad 140 \quad 120 \quad 100 \quad 80 \quad 60 \quad 40 \quad 20 \begin{array}{lllllllllllll}0 & 0 & -10 & -30 & -50 & -70 & -90 & -120 & -150 & -180 & \end{array}$

$376 \mathrm{MHz}{ }^{19} \mathrm{~F}$ NMR spectra of compound 4ea in $\mathrm{CDCl}_{3}$ 

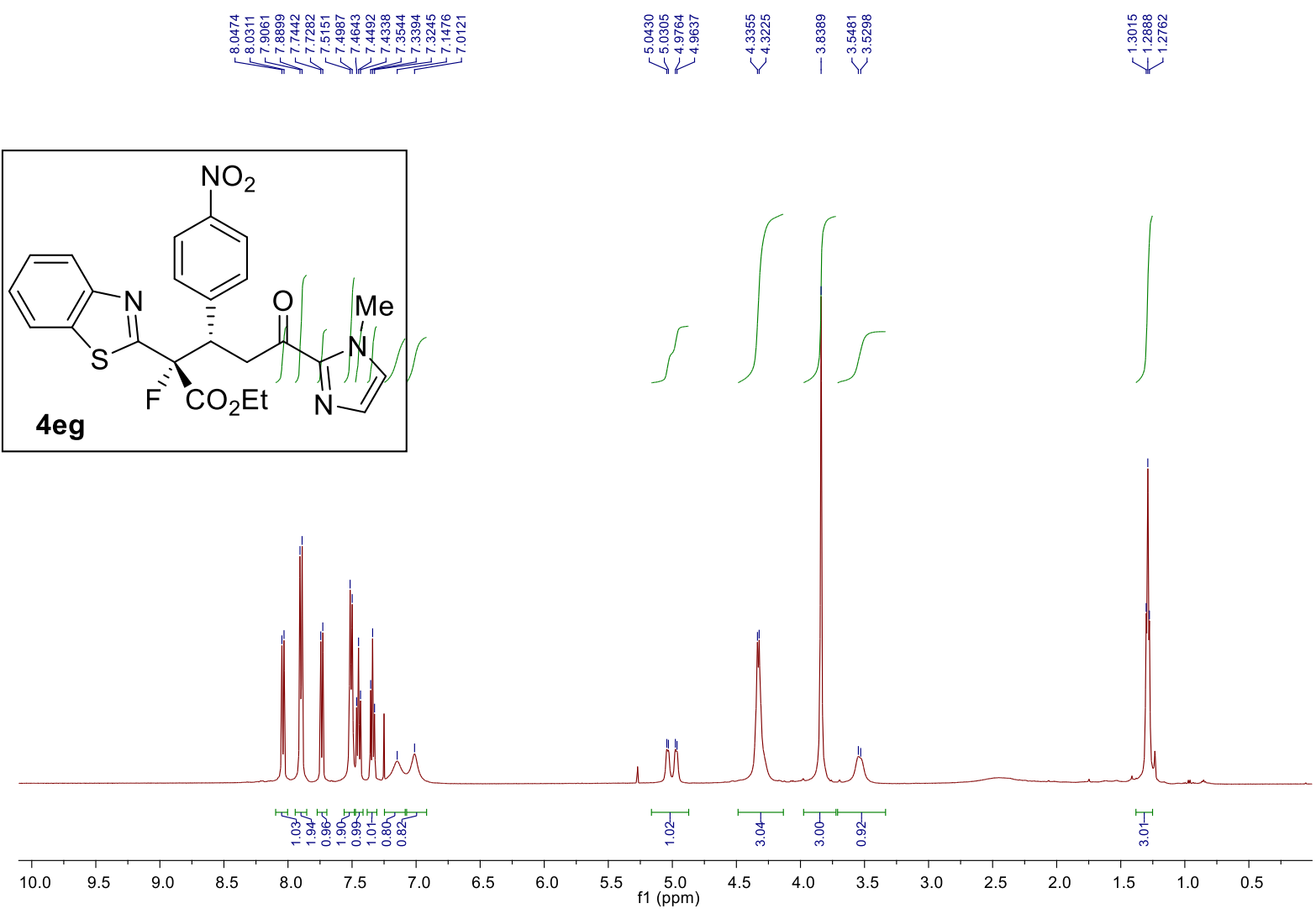

$500 \mathrm{MHz}{ }^{1} \mathrm{H}$ NMR spectra of compound $4 \mathrm{eg}$ in $\mathrm{CDCl}_{3}$

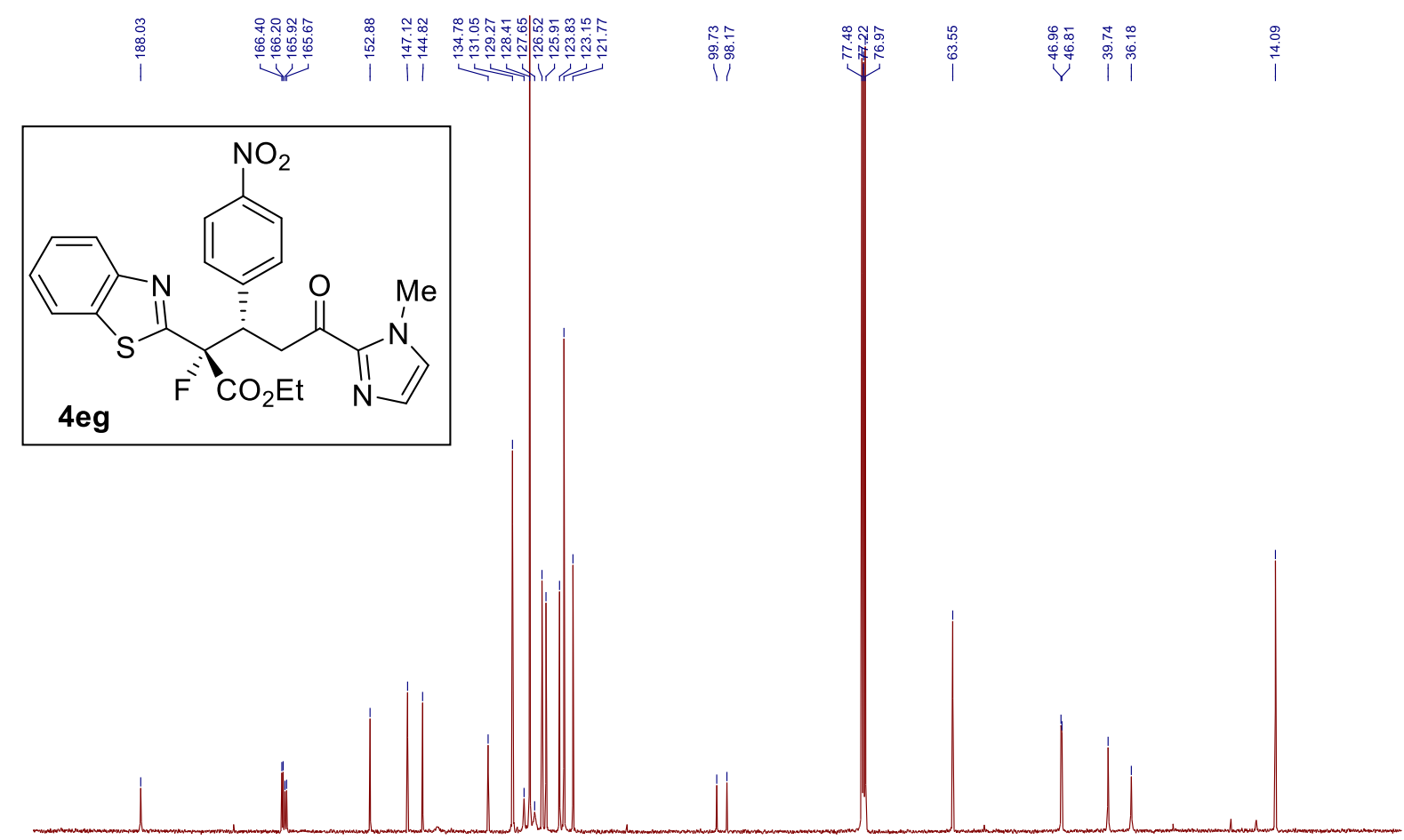

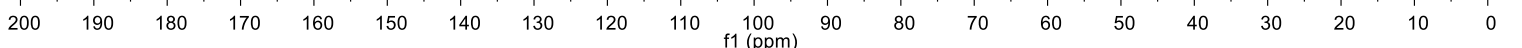

$125 \mathrm{MHz}{ }^{13} \mathrm{C}$ NMR spectra of compound $4 \mathrm{eg}$ in $\mathrm{CDCl}_{3}$ 

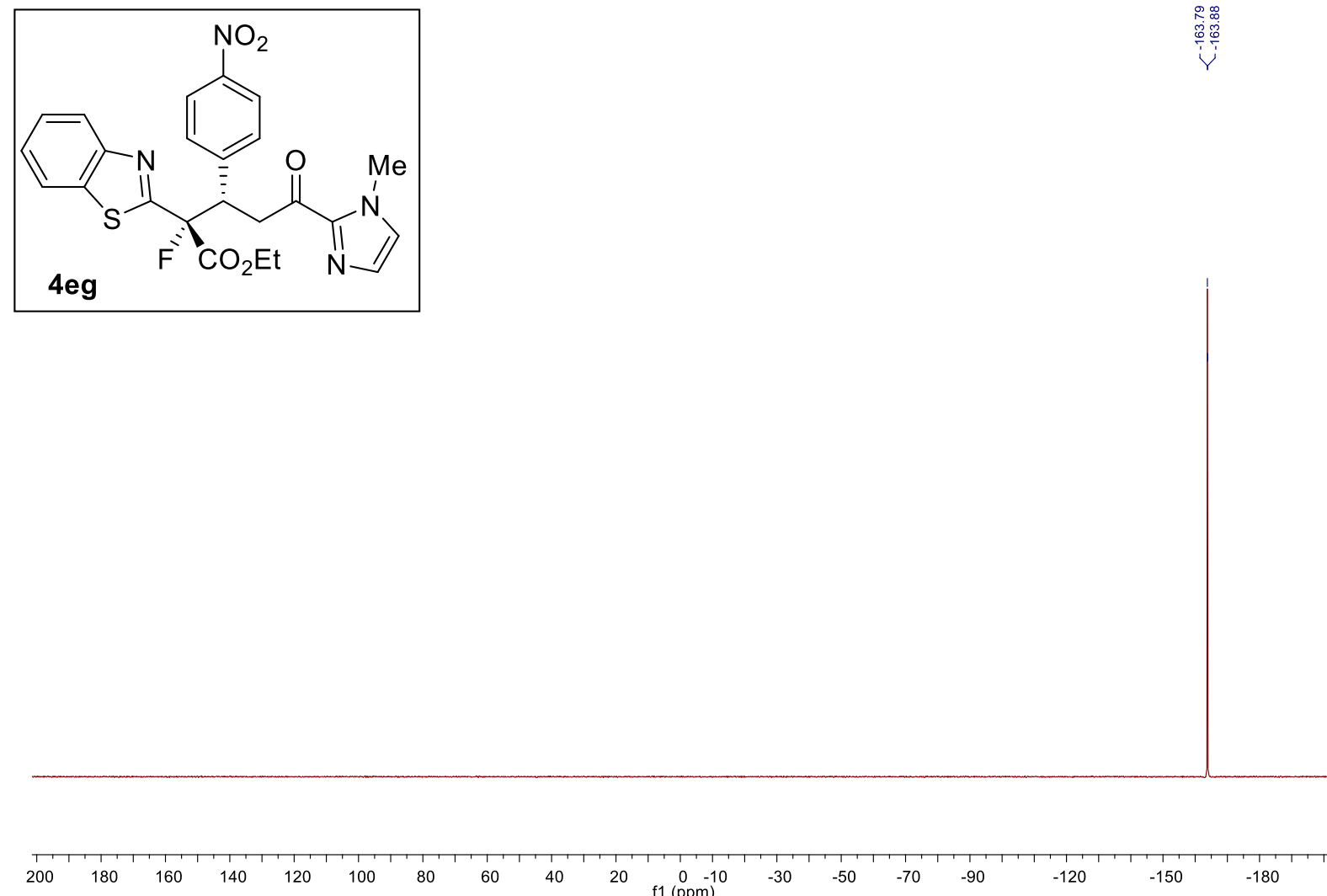

$376 \mathrm{MHz}^{19} \mathrm{~F}$ NMR spectra of compound $4 \mathrm{eg}$ in $\mathrm{CDCl}_{3}$ 

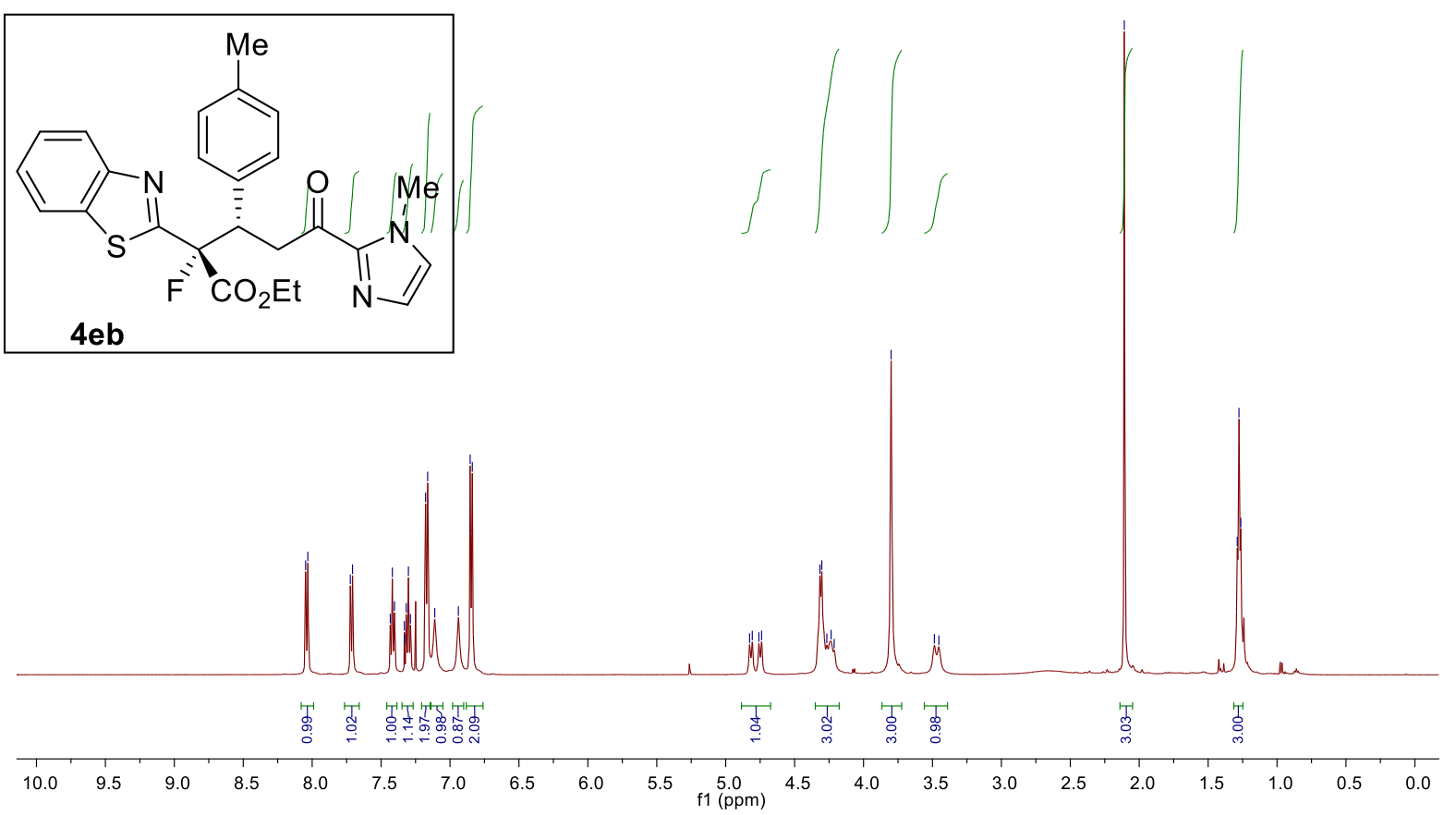

$500 \mathrm{MHz}{ }^{1} \mathrm{H}$ NMR spectra of compound $4 \mathrm{eb}$ in $\mathrm{CDCl}_{3}$

\begin{tabular}{|c|c|c|c|c|c|c|c|c|}
\hline 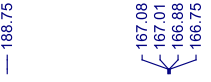 & $\overbrace{\substack{\infty \\
\sim}}^{\infty}$ & 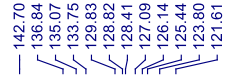 & 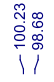 & $\underbrace{\text { inj }}$ & $\stackrel{\substack{\dot{0} \\
0}}{1}$ & 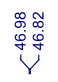 & & $\stackrel{\stackrel{\leftrightarrow}{\pi}}{i}$ \\
\hline
\end{tabular}
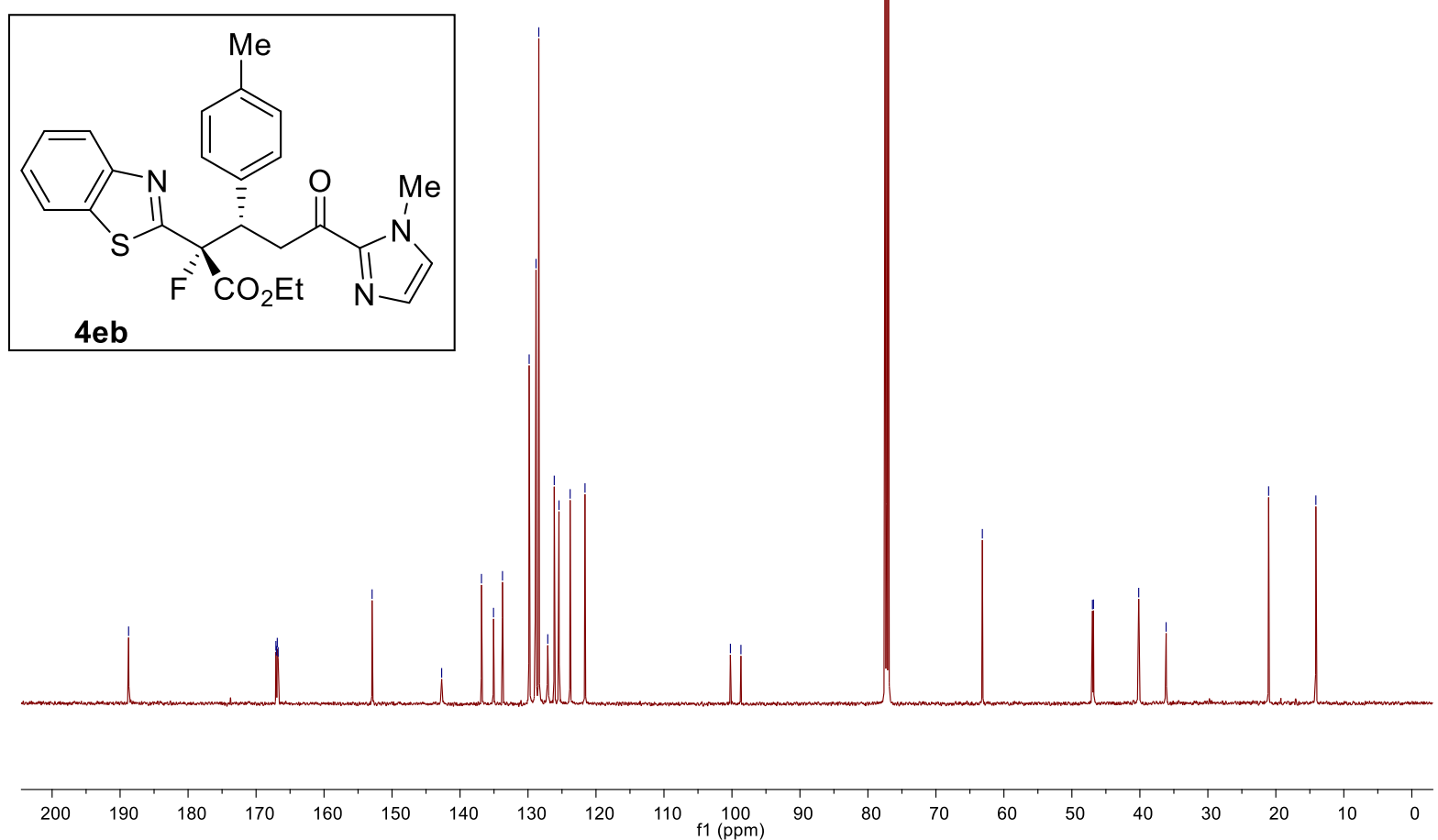

$125 \mathrm{MHz}{ }^{13} \mathrm{C}$ NMR spectra of compound $4 \mathrm{eb}$ in $\mathrm{CDCl}_{3}$ 


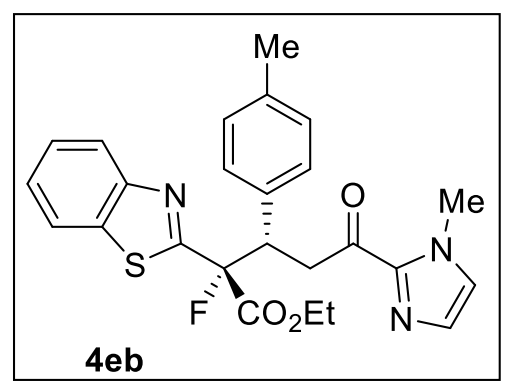

$\begin{array}{llllllllllllllllllllll} & 200 & 180 & 160 & 140 & 120 & 100 & 80 & 60 & 40 & 20 & 0 & 0 & -10 & -30 & -50 & -70 & -90 & -120 & -150 & -180 & \end{array}$

$376 \mathrm{MHz}^{19} \mathrm{~F}$ NMR spectra of compound $4 \mathrm{eb}$ in $\mathrm{CDCl}_{3}$ 


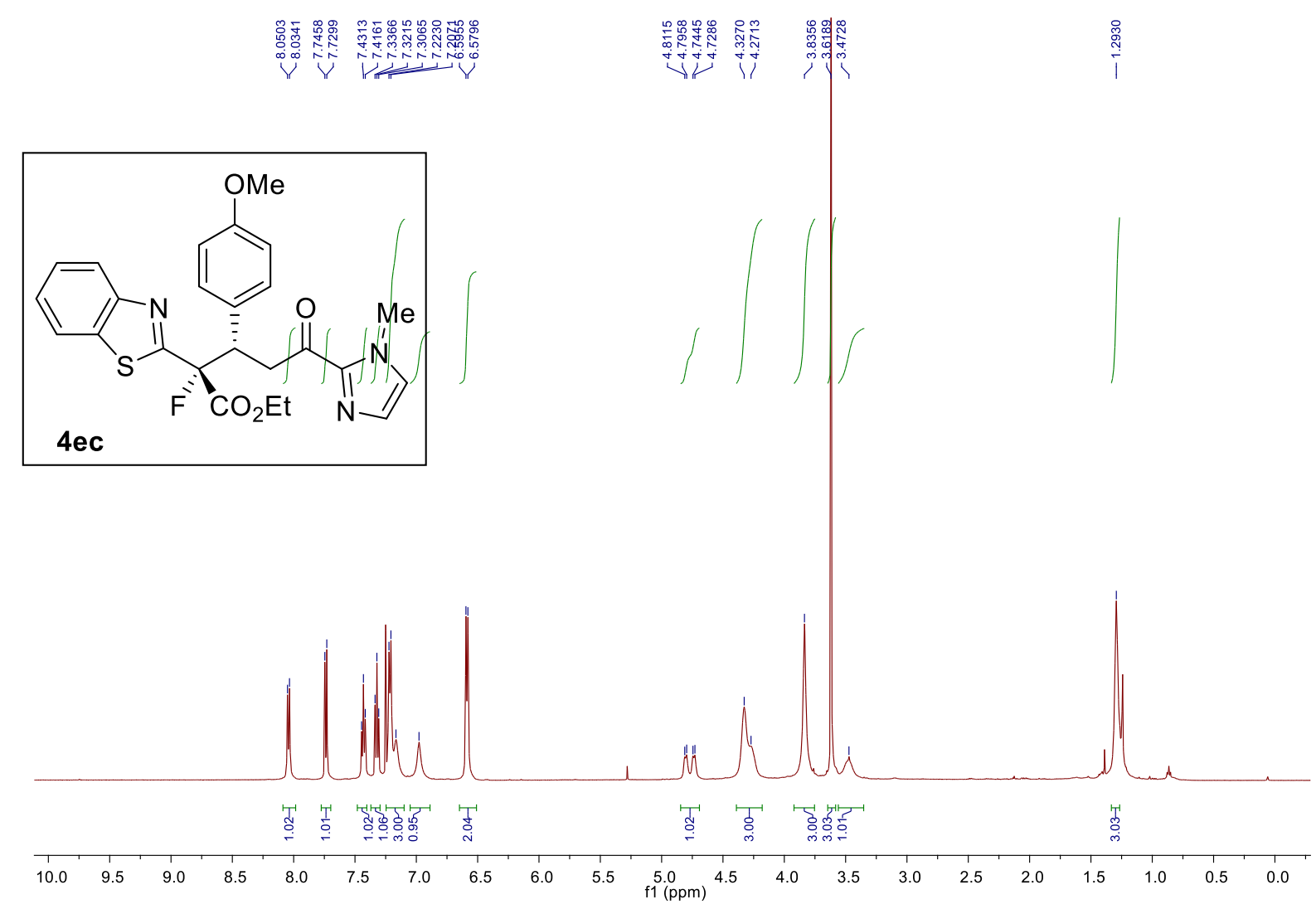

$500 \mathrm{MHz}{ }^{1} \mathrm{H}$ NMR spectra of compound $4 \mathrm{ec}$ in $\mathrm{CDCl}_{3}$
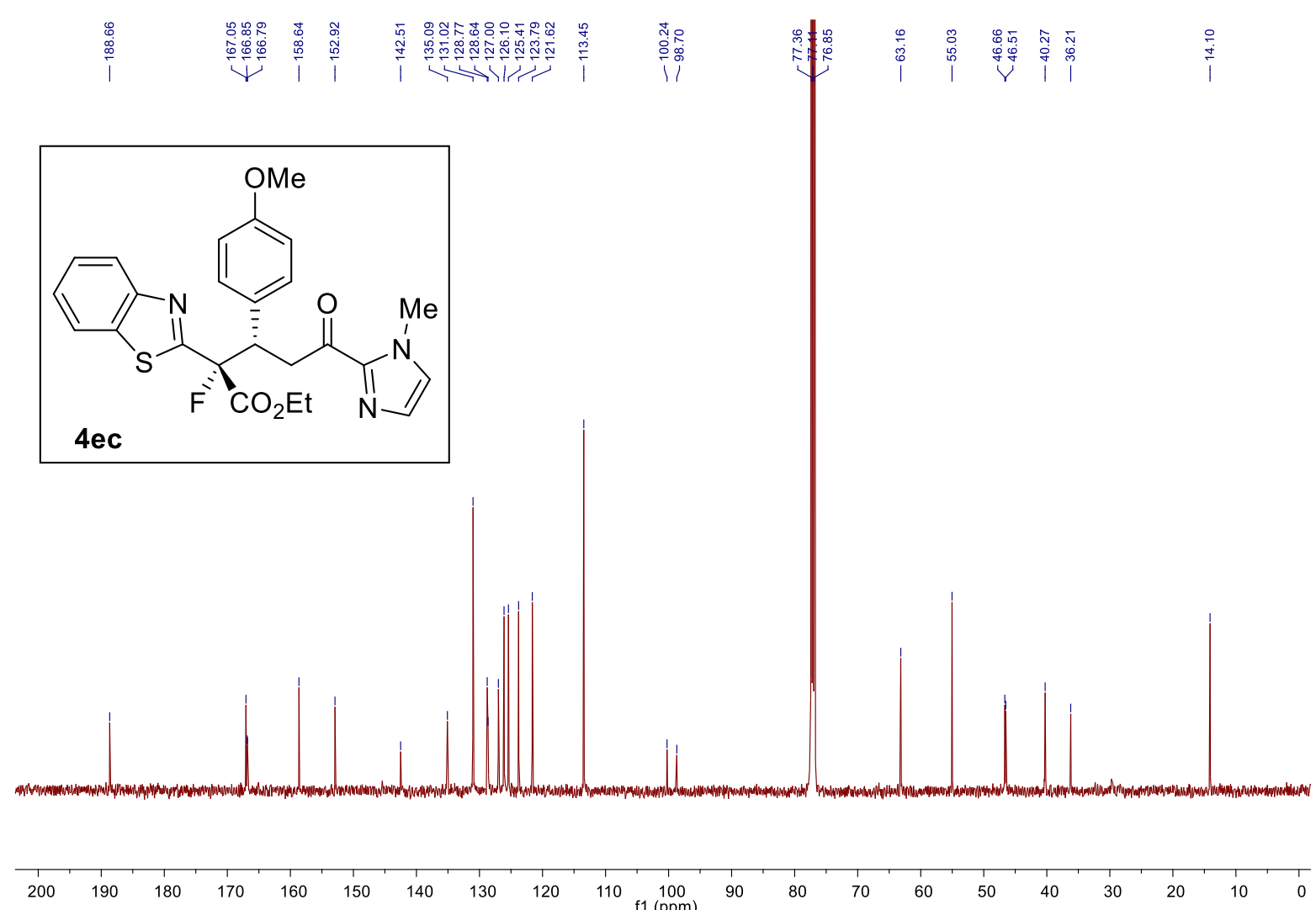

$125 \mathrm{MHz}{ }^{13} \mathrm{C}$ NMR spectra of compound $4 \mathrm{ec}$ in $\mathrm{CDCl}_{3}$ 


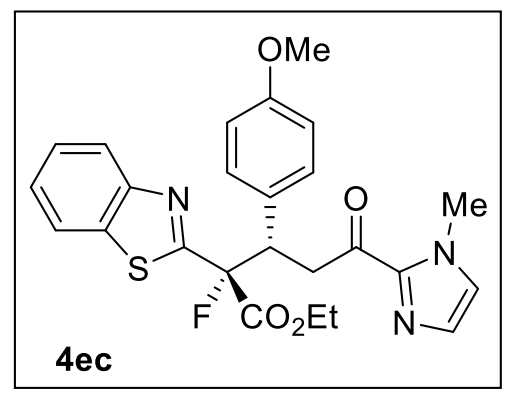

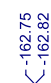

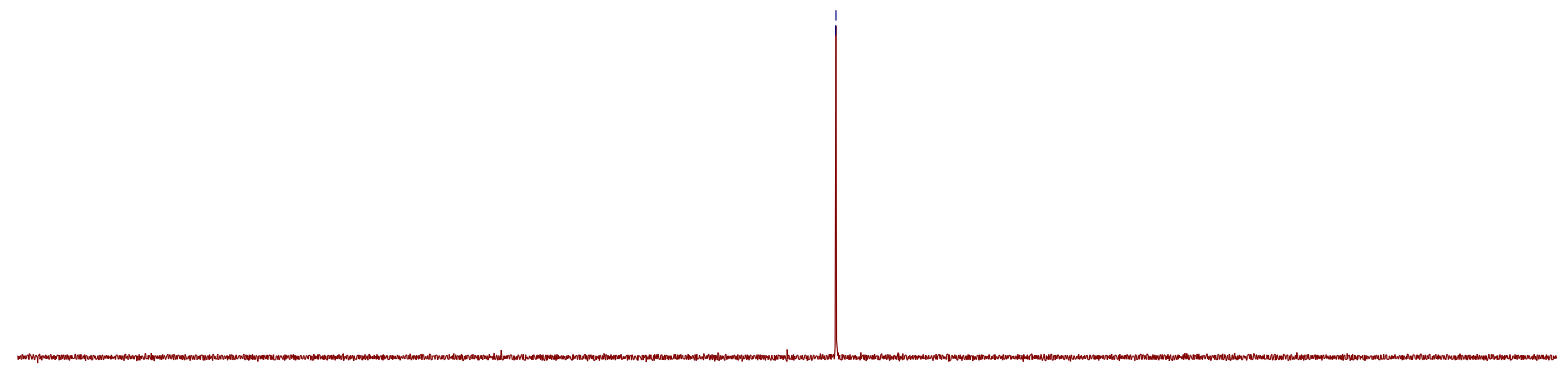

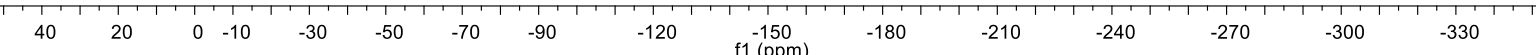

$376 \mathrm{MHz}{ }^{19} \mathrm{~F}$ NMR spectra of compound $4 \mathrm{ec}$ in $\mathrm{CDCl}_{3}$ 

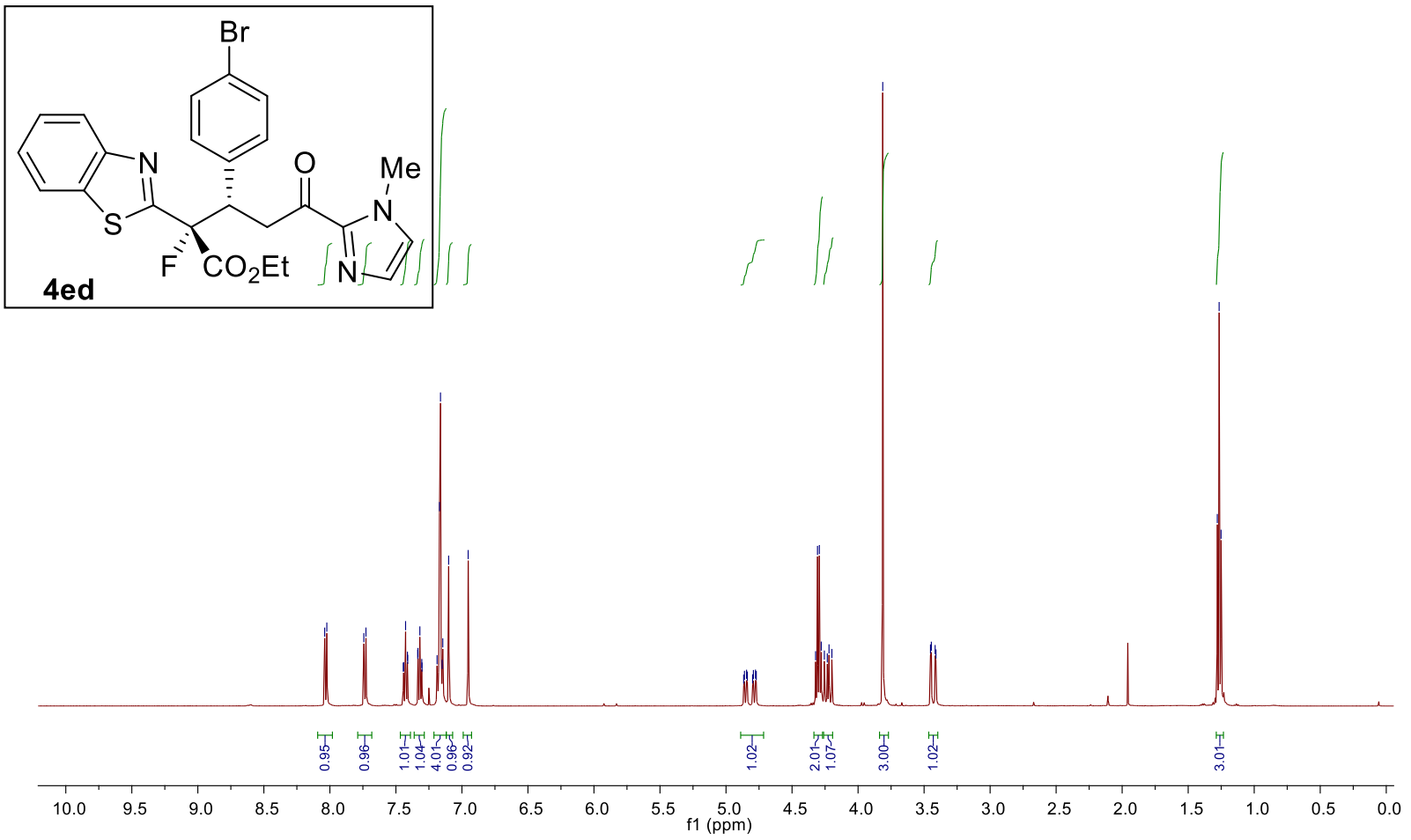

$500 \mathrm{MHz}{ }^{1} \mathrm{H}$ NMR spectra of compound 4 ed in $\mathrm{CDCl}_{3}$

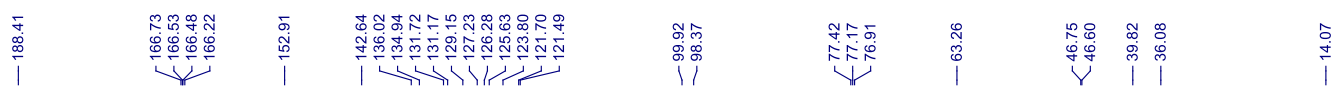
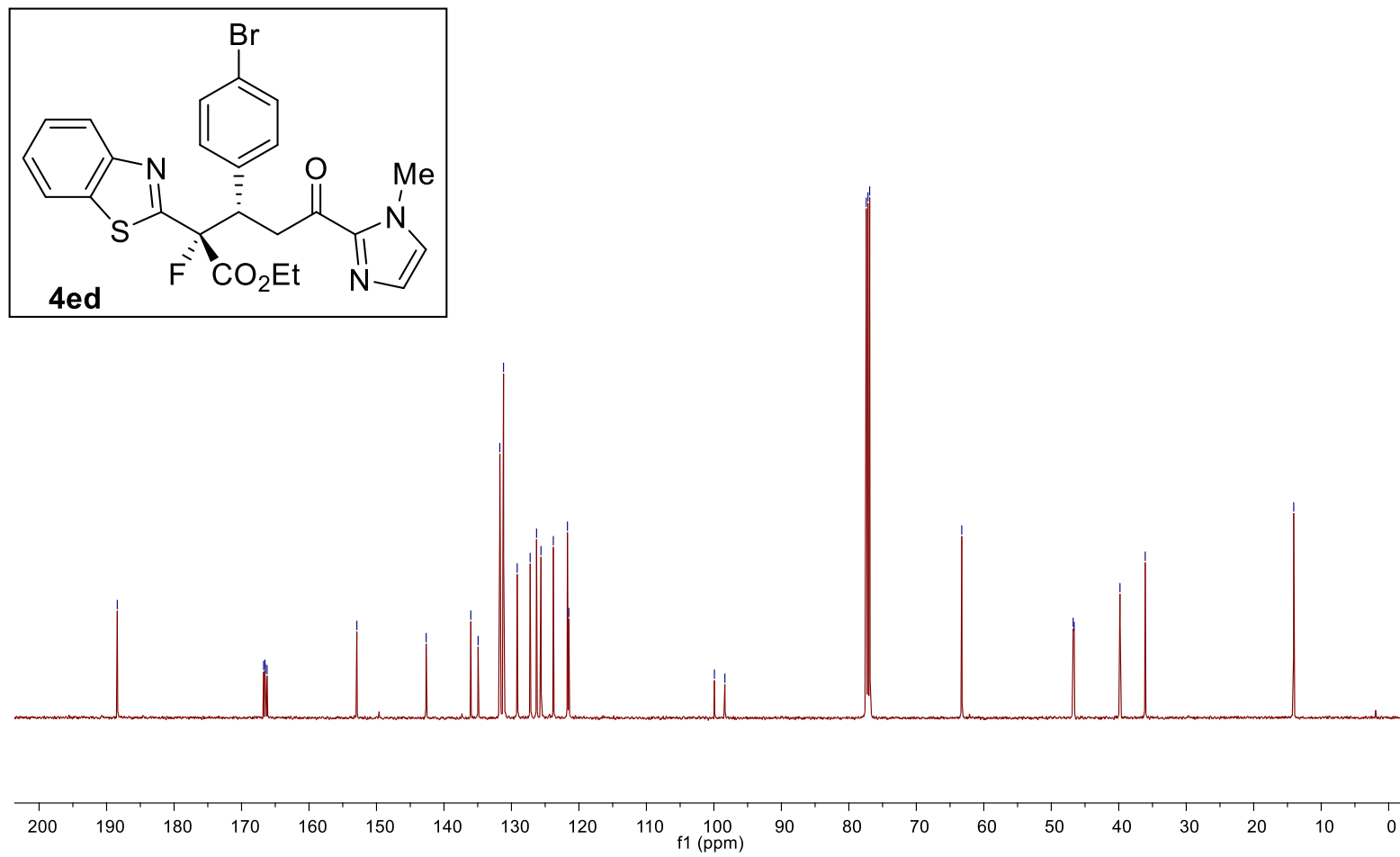

$125 \mathrm{MHz}{ }^{13} \mathrm{C}$ NMR spectra of compound $4 \mathrm{ed}$ in $\mathrm{CDCl}_{3}$ 

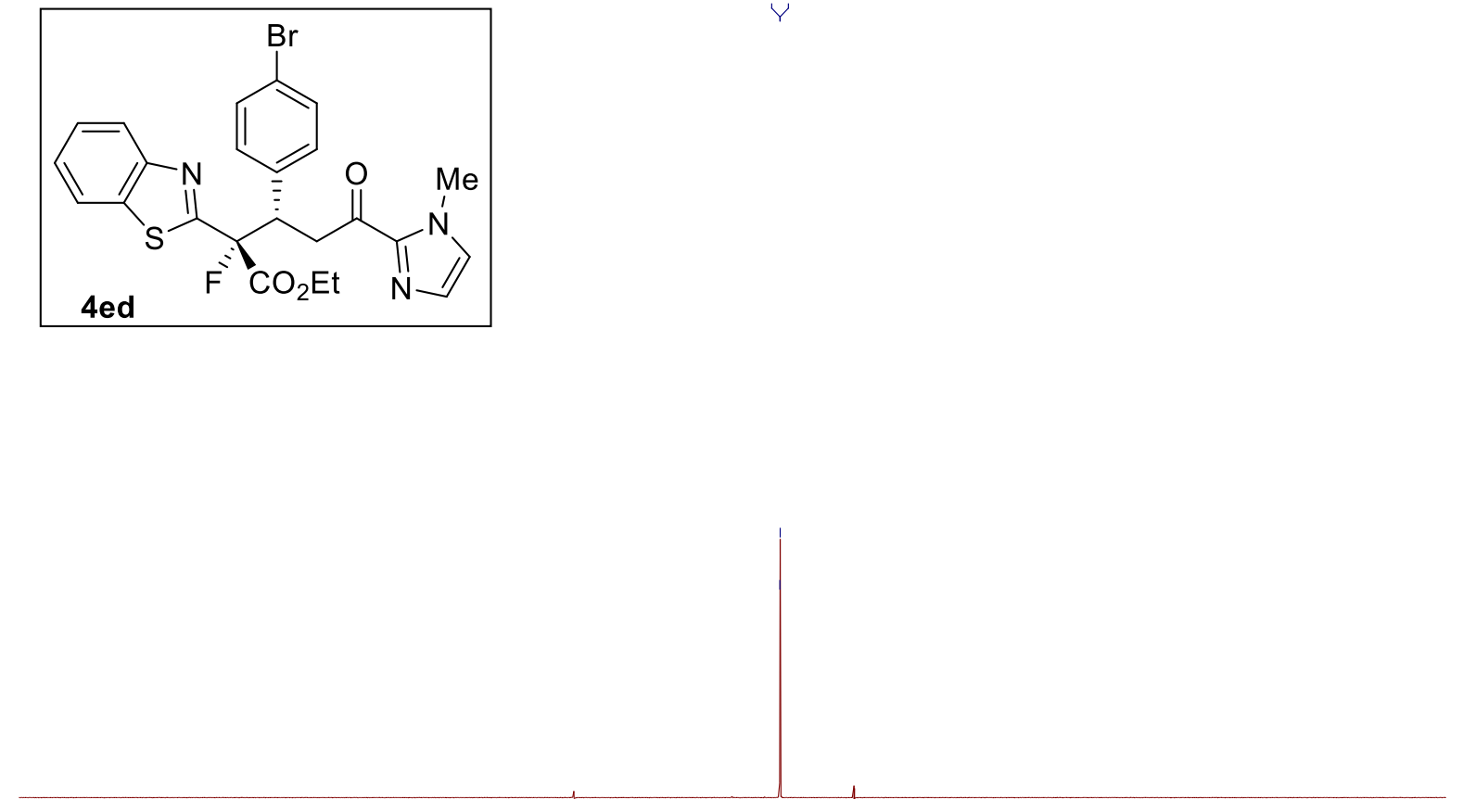

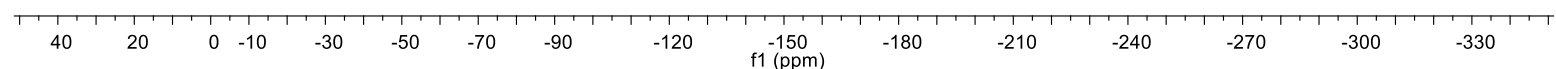

$376 \mathrm{MHz}^{19} \mathrm{~F}$ NMR spectra of compound $4 \mathrm{ed}$ in $\mathrm{CDCl}_{3}$ 

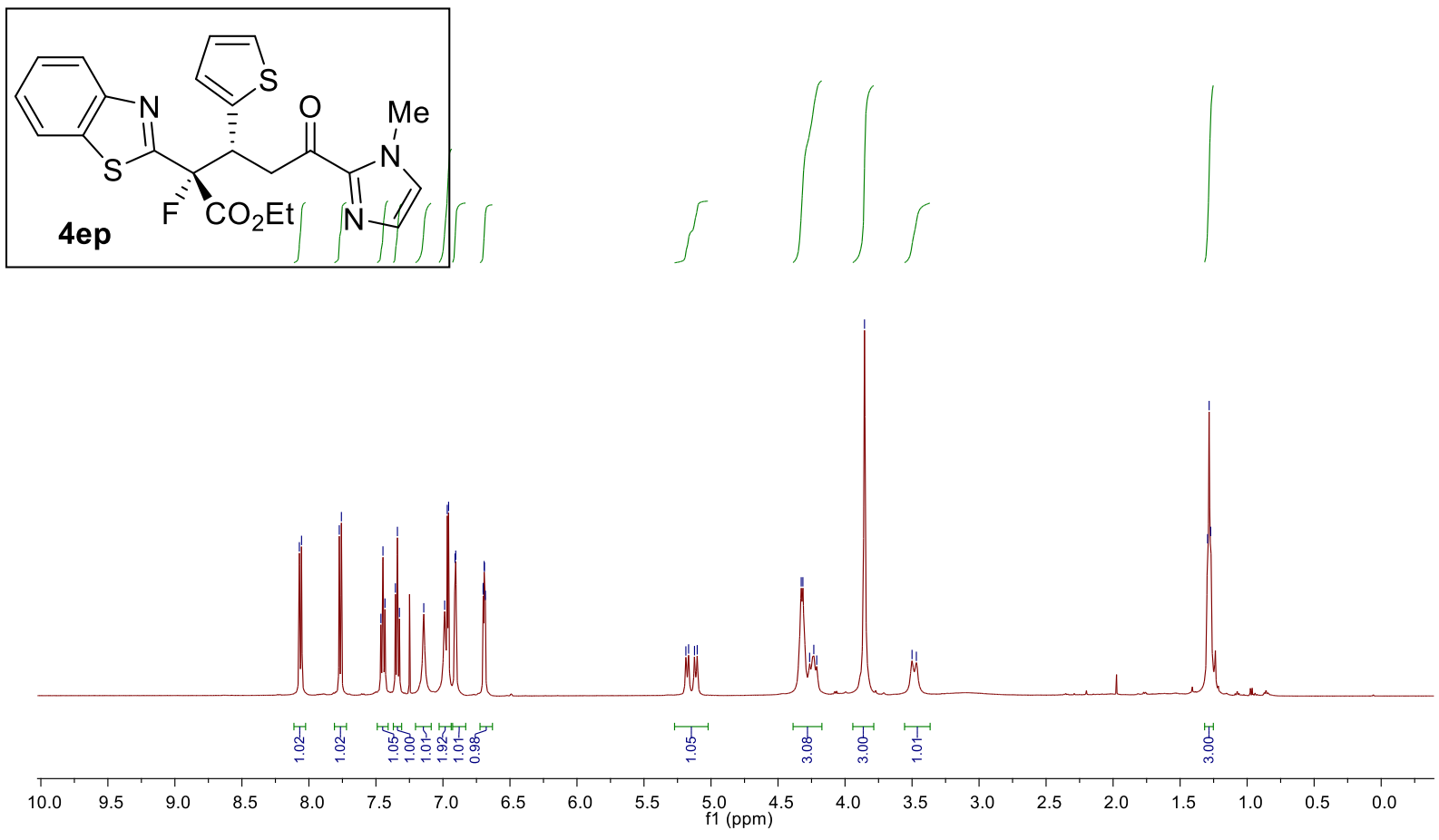

$500 \mathrm{MHz}{ }^{1} \mathrm{H}$ NMR spectra of compound 4ep in $\mathrm{CDCl}_{3}$

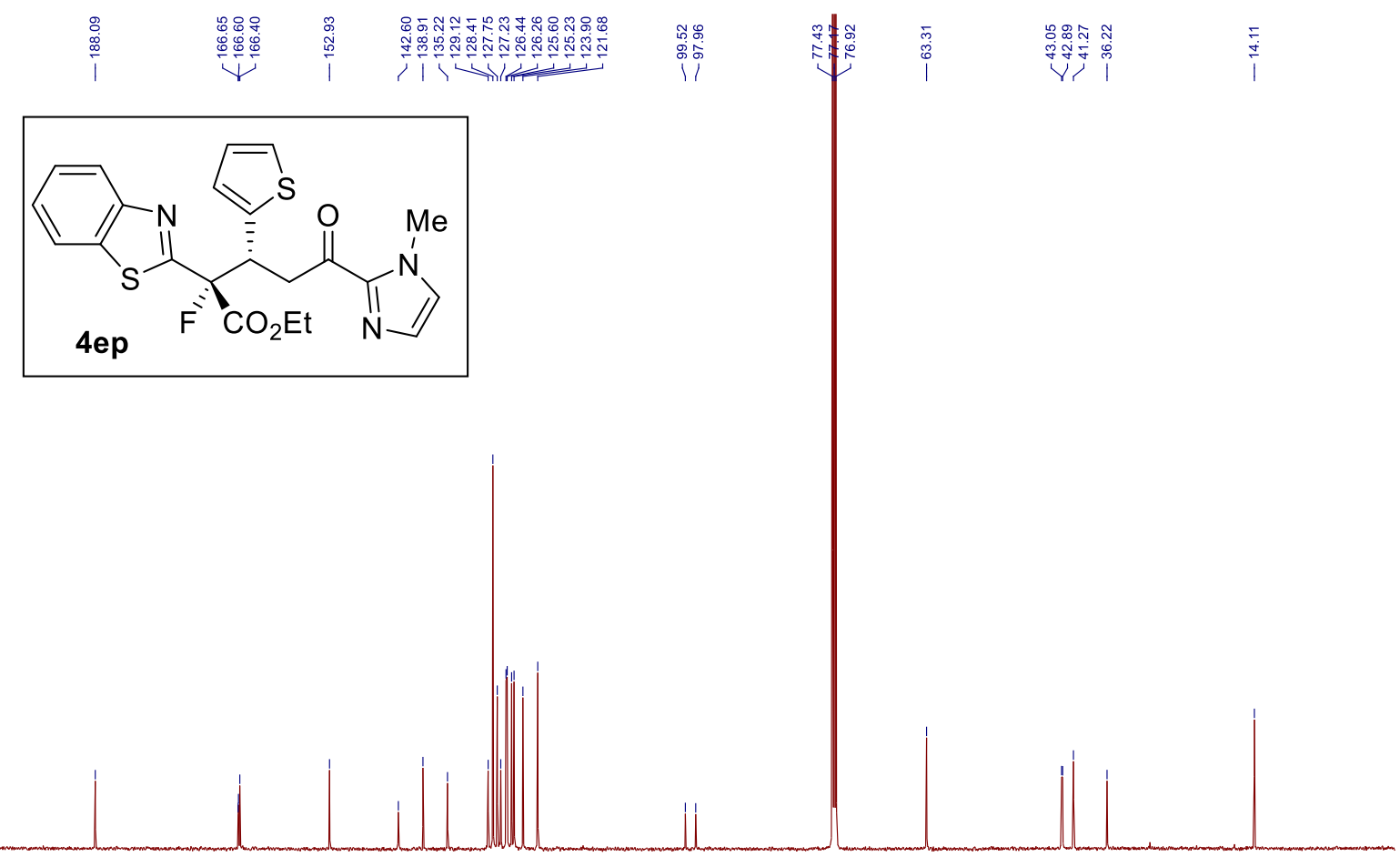

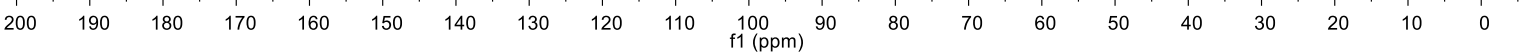

$125 \mathrm{MHz}{ }^{13} \mathrm{C}$ NMR spectra of compound 4ep in $\mathrm{CDCl}_{3}$ 

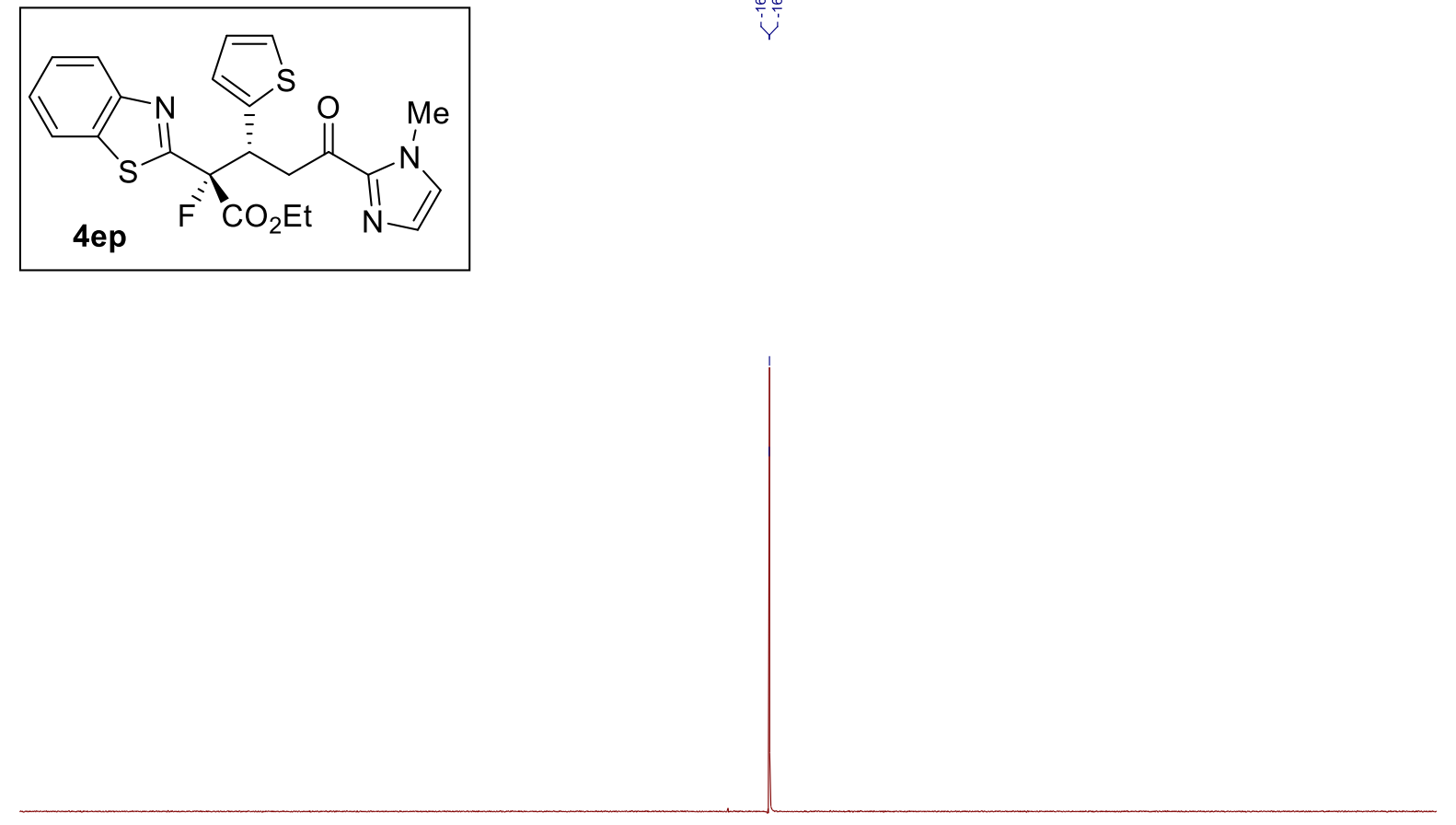

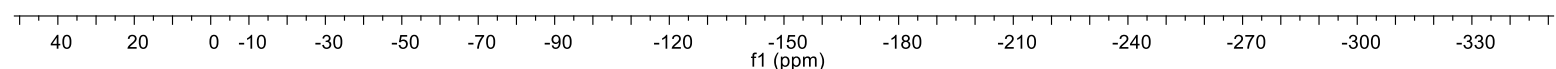

$376 \mathrm{MHz}{ }^{19} \mathrm{~F}$ NMR spectra of compound 4ep in $\mathrm{CDCl}_{3}$ 

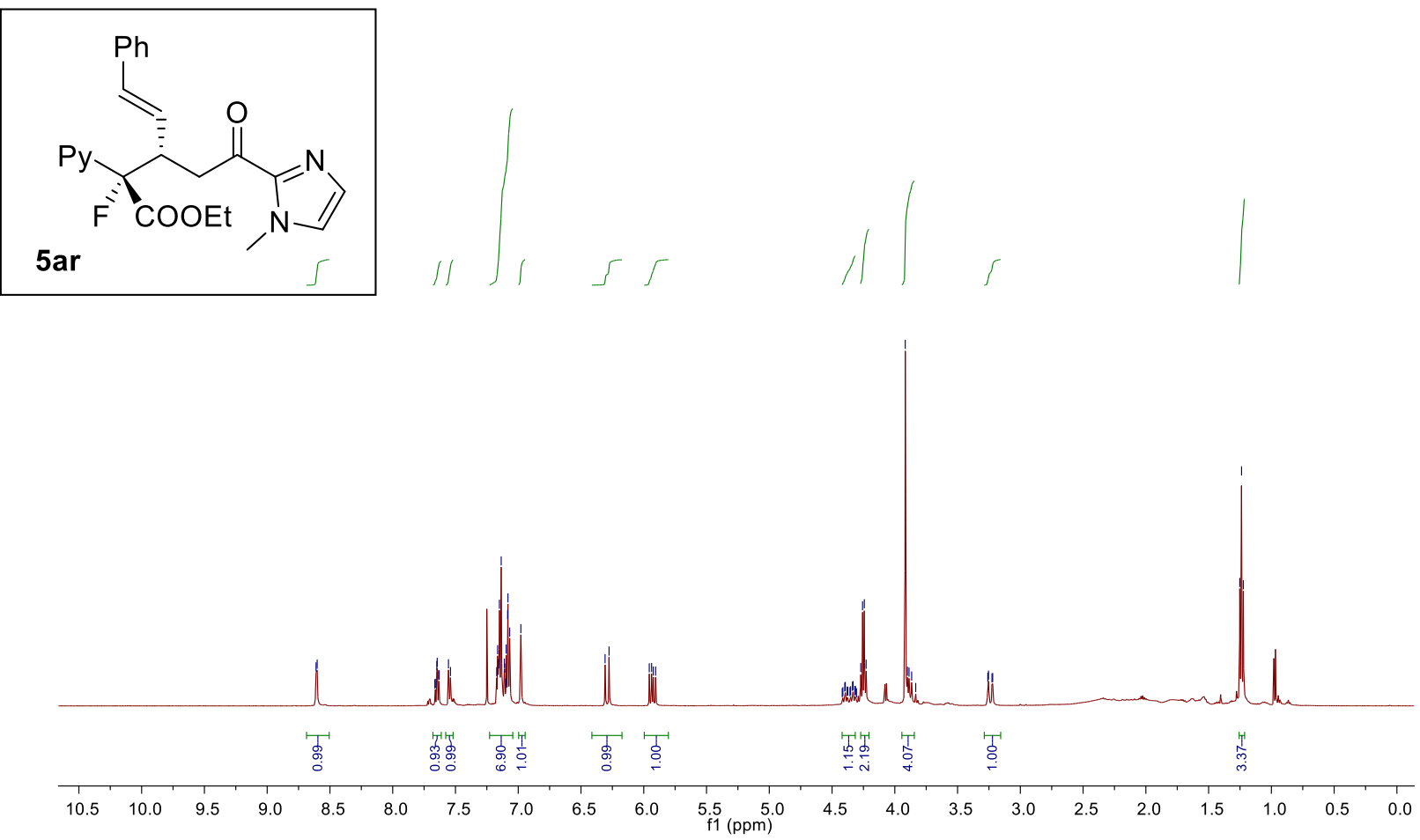

$500 \mathrm{MHz}{ }^{1} \mathrm{H}$ NMR spectra of compound 5ar in $\mathrm{CDCl}_{3}$

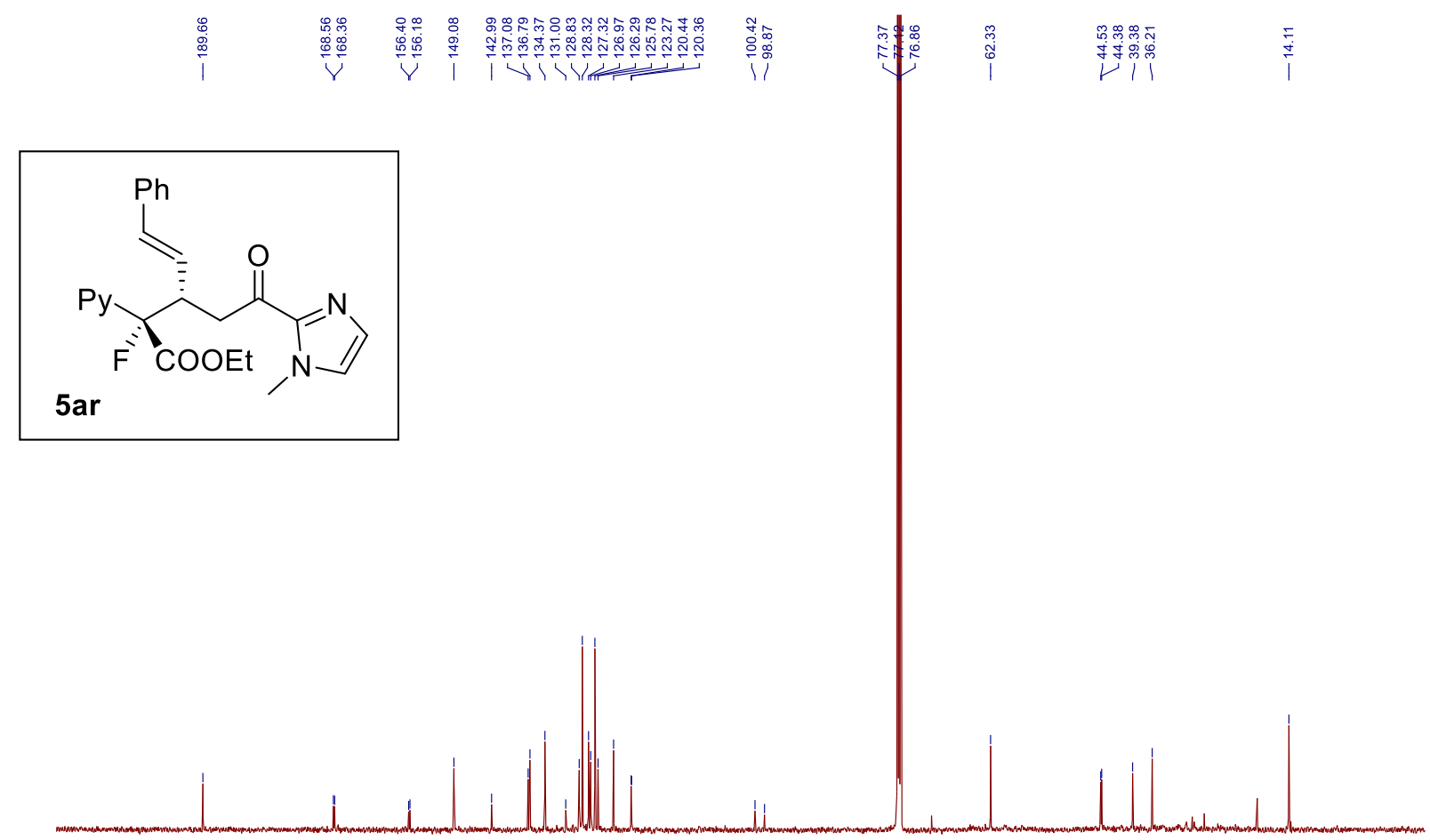

$\begin{array}{llllllllllll}210 & 200 & 190 & 180 & 170 & 160 & 150 & 140 & 130 & 120 & 110 & 100\end{array}$

$125 \mathrm{MHz}{ }^{13} \mathrm{C}$ NMR spectra of compound 5 ar in $\mathrm{CDCl}_{3}$ 


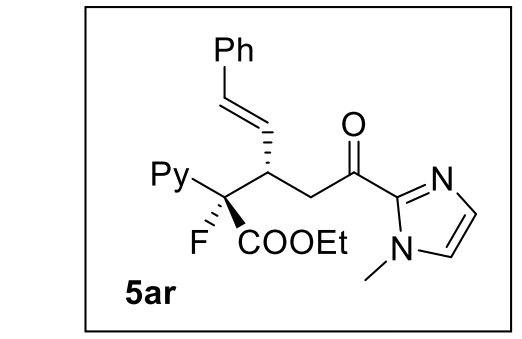

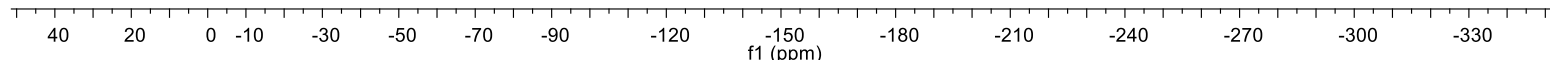

$376 \mathrm{MHz}{ }^{19} \mathrm{~F}$ NMR spectra of compound 5 ar in $\mathrm{CDCl}_{3}$ 

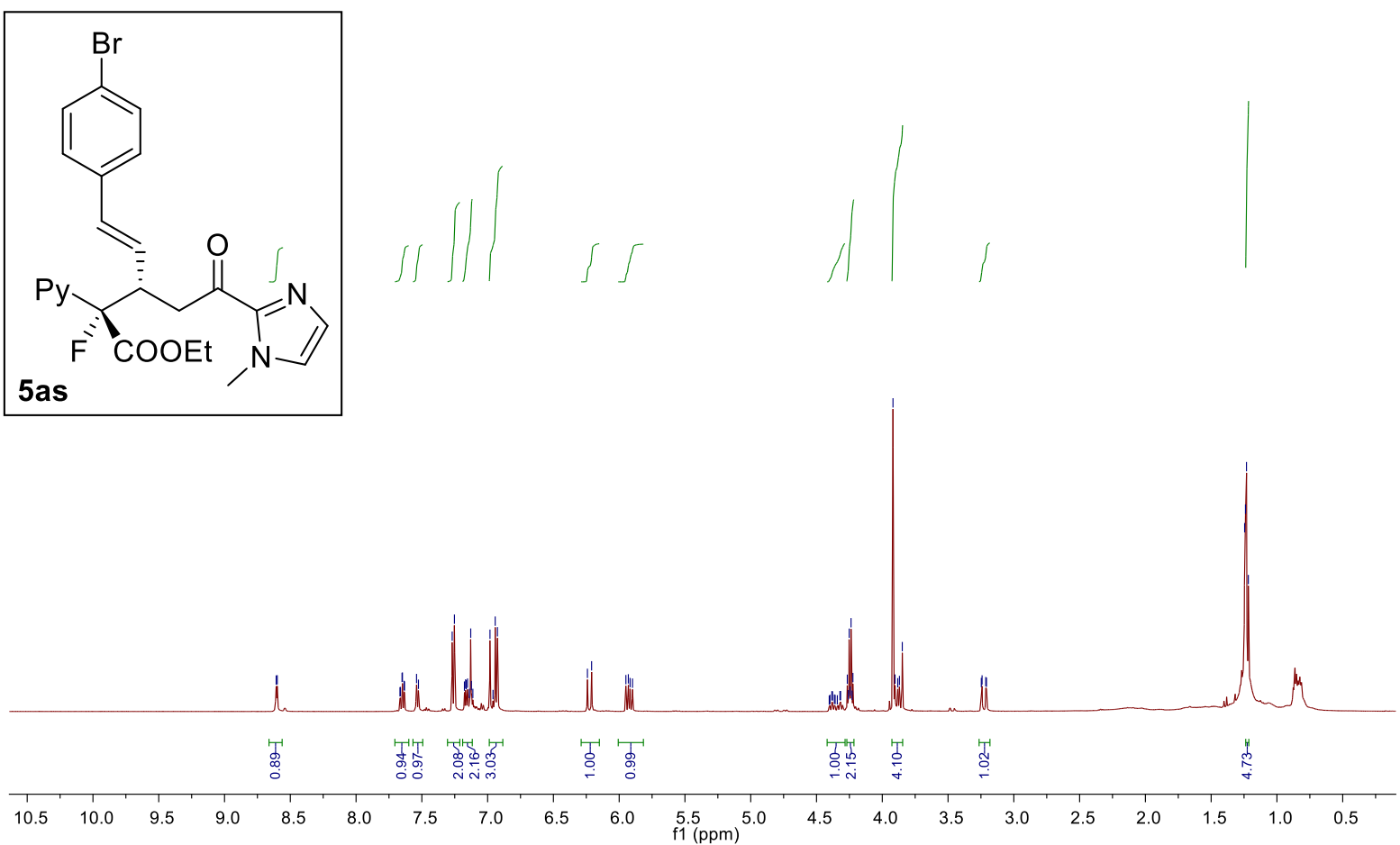

$500 \mathrm{MHz}{ }^{1} \mathrm{H}$ NMR spectra of compound 5 as in $\mathrm{CDCl}_{3}$

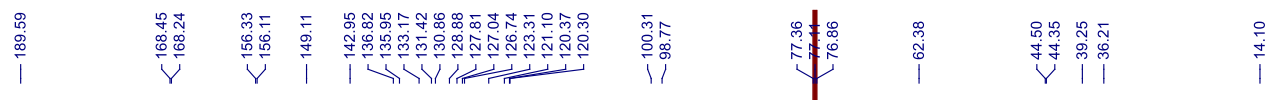<smiles>CCOC(=O)C(F)(C(=O)OCC)[C@H](/C=C/c1ccc(Br)cc1)CC(=O)c1nccn1C</smiles>

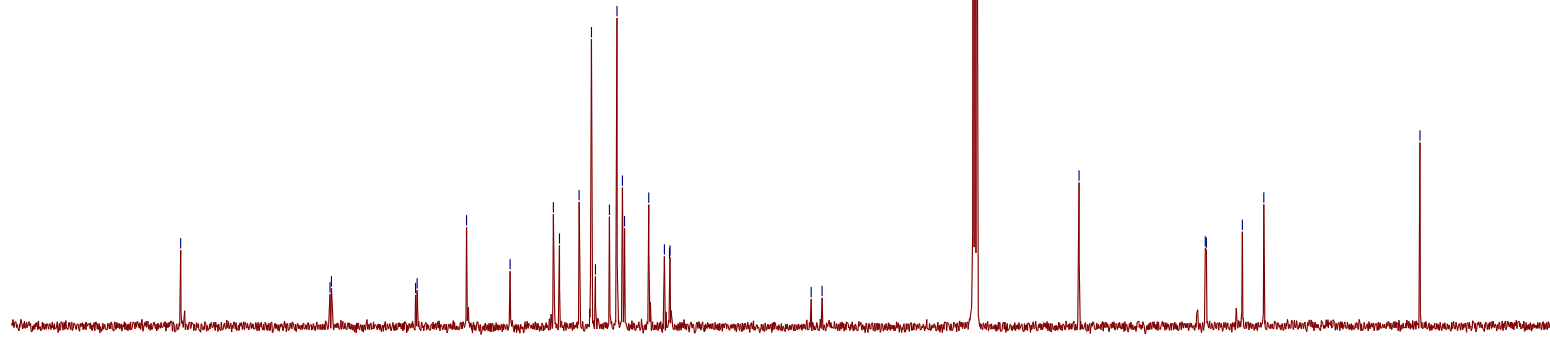

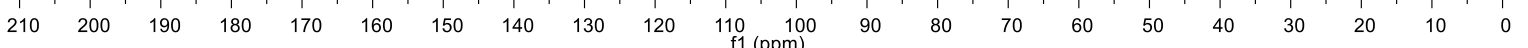

$125 \mathrm{MHz}{ }^{13} \mathrm{C}$ NMR spectra of compound 5as in $\mathrm{CDCl}_{3}$ 


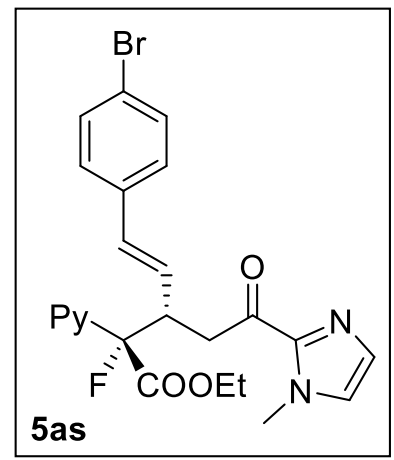

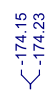

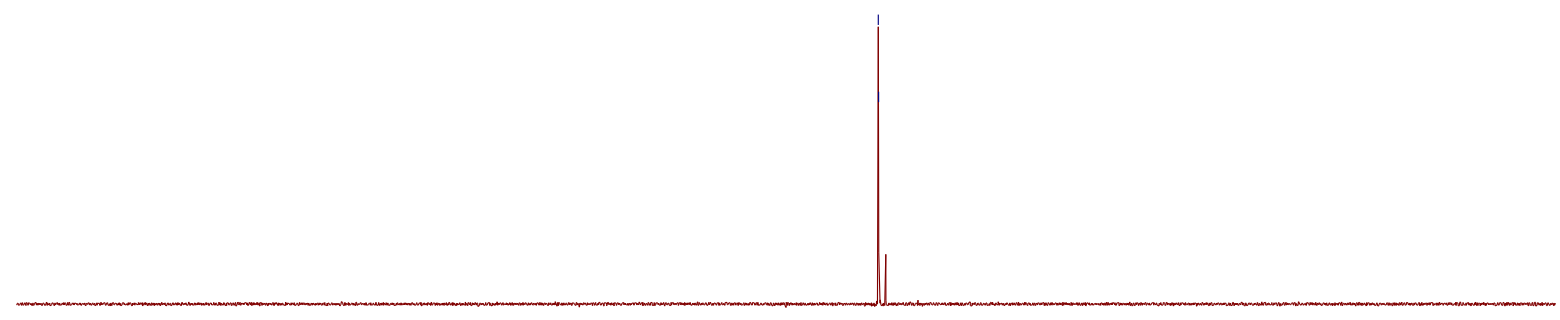

$\begin{array}{llllllllllllllll} & \\ 40 & 20 & 0 & -10 & -30 & -50 & -70 & -90 & -120 & -150 & -180 & -210 & -240 & -270 & -300 & -330\end{array}$

$376 \mathrm{MHz}^{19} \mathrm{~F}$ NMR spectra of compound 5 as in $\mathrm{CDCl}_{3}$ 


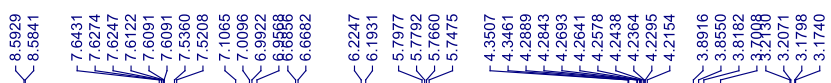
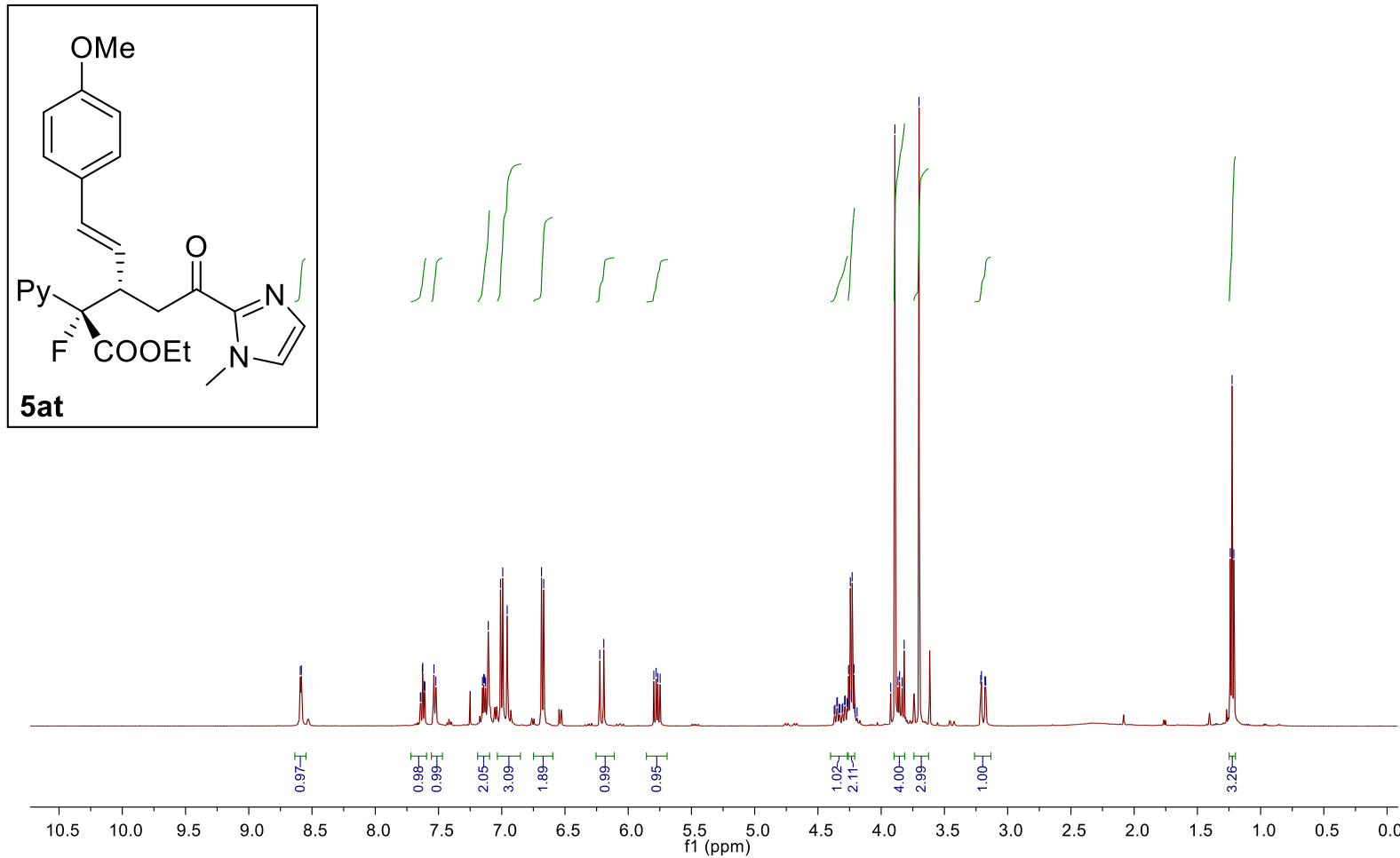

$500 \mathrm{MHz}{ }^{1} \mathrm{H}$ NMR spectra of compound 5at in $\mathrm{CDCl}_{3}$

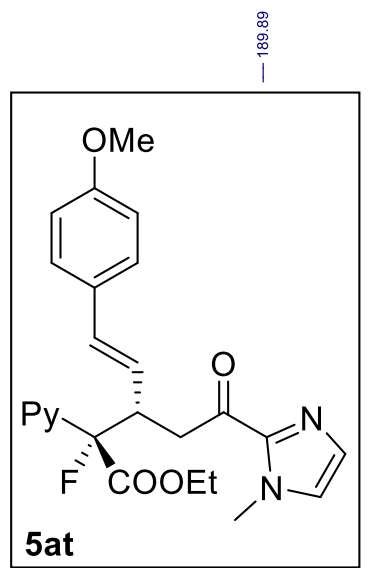

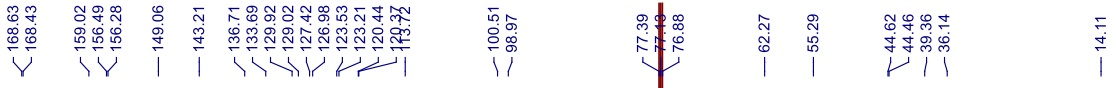

5at

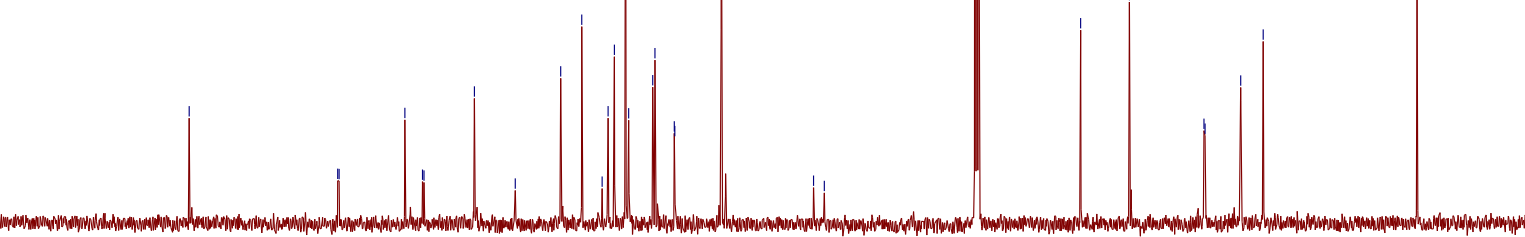

$\begin{array}{lllllllllll}210 & 200 & 190 & 180 & 170 & 160 & 150 & 140 & 130 & 120 & \begin{array}{l}110 \\ \mathrm{f} 1(\mathrm{ppm})\end{array}\end{array}$

$125 \mathrm{MHz}{ }^{13} \mathrm{C}$ NMR spectra of compound 5 at in $\mathrm{CDCl}_{3}$ 


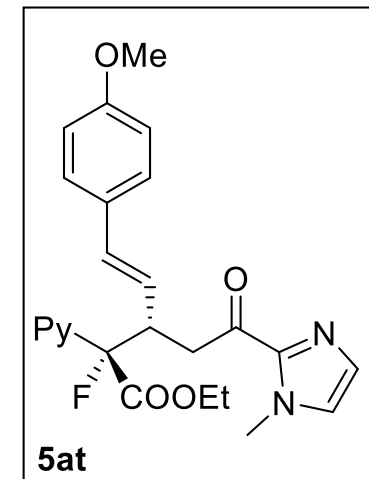

$200 \quad 180 \quad 160 \quad 140 \quad 120 \quad 100 \quad 80 \quad 60 \quad 40 \quad \begin{array}{lllllllllllll}0 & 0 & -10 & -30 & -50 & -70 & -90 & -120 & -150 & -180\end{array}$ $376 \mathrm{MHz}{ }^{19} \mathrm{~F}$ NMR spectra of compound 5at in $\mathrm{CDCl}_{3}$ 


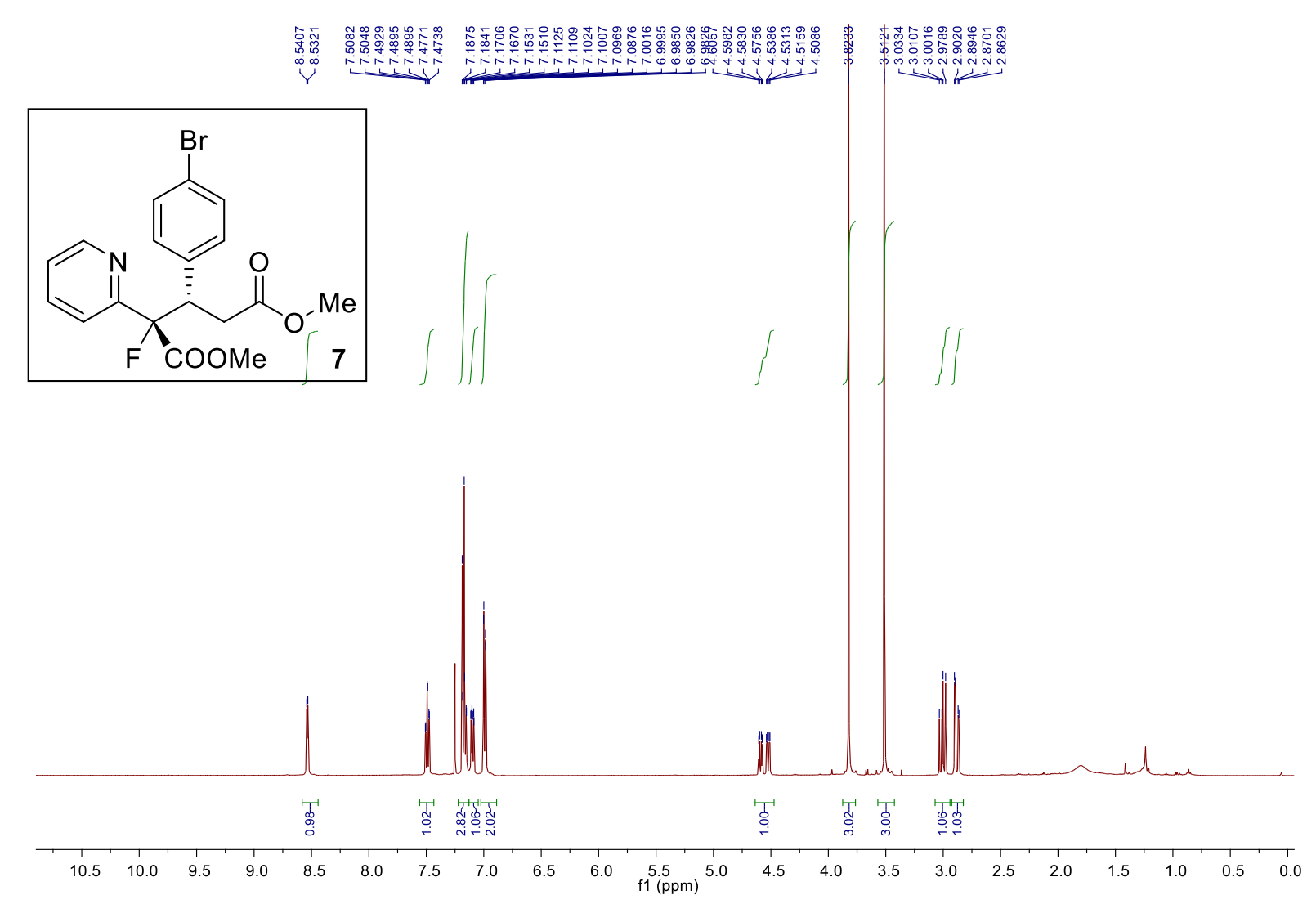

$500 \mathrm{MHz}{ }^{1} \mathrm{H}$ NMR spectra of compound 7 in $\mathrm{CDCl}_{3}$

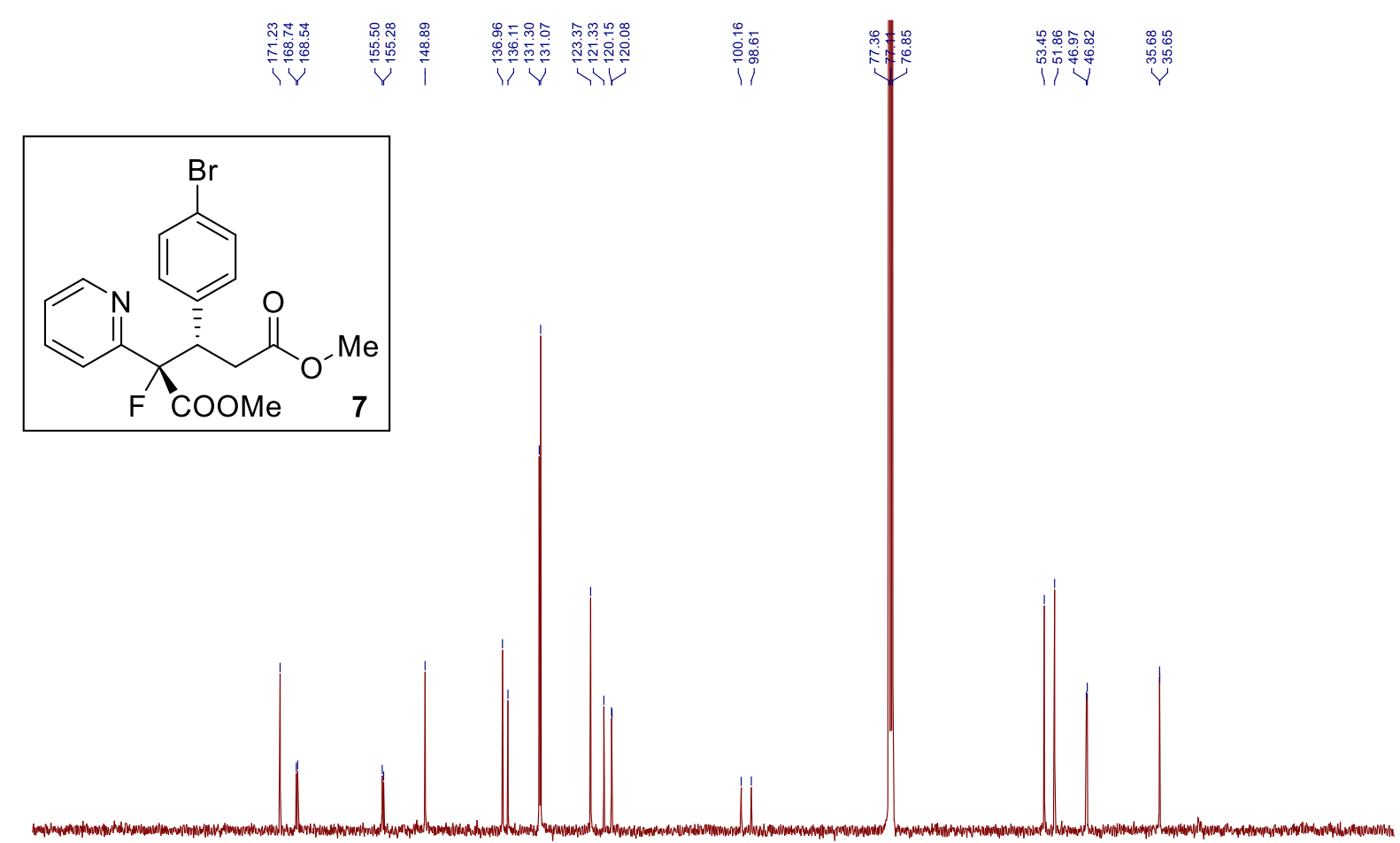

$\begin{array}{llllllllll}200 & 190 & 180 & 170 & 160 & 150 & 140 & 130 & 120 & \begin{array}{c}110 \\ \mathrm{f} 1(\mathrm{ppm})\end{array}\end{array}$

$125 \mathrm{MHz}{ }^{13} \mathrm{C}$ NMR spectra of compound 7 in $\mathrm{CDCl}_{3}$ 


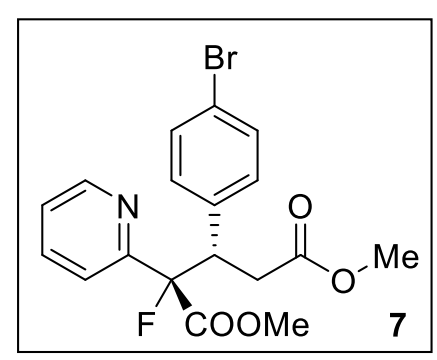

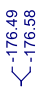

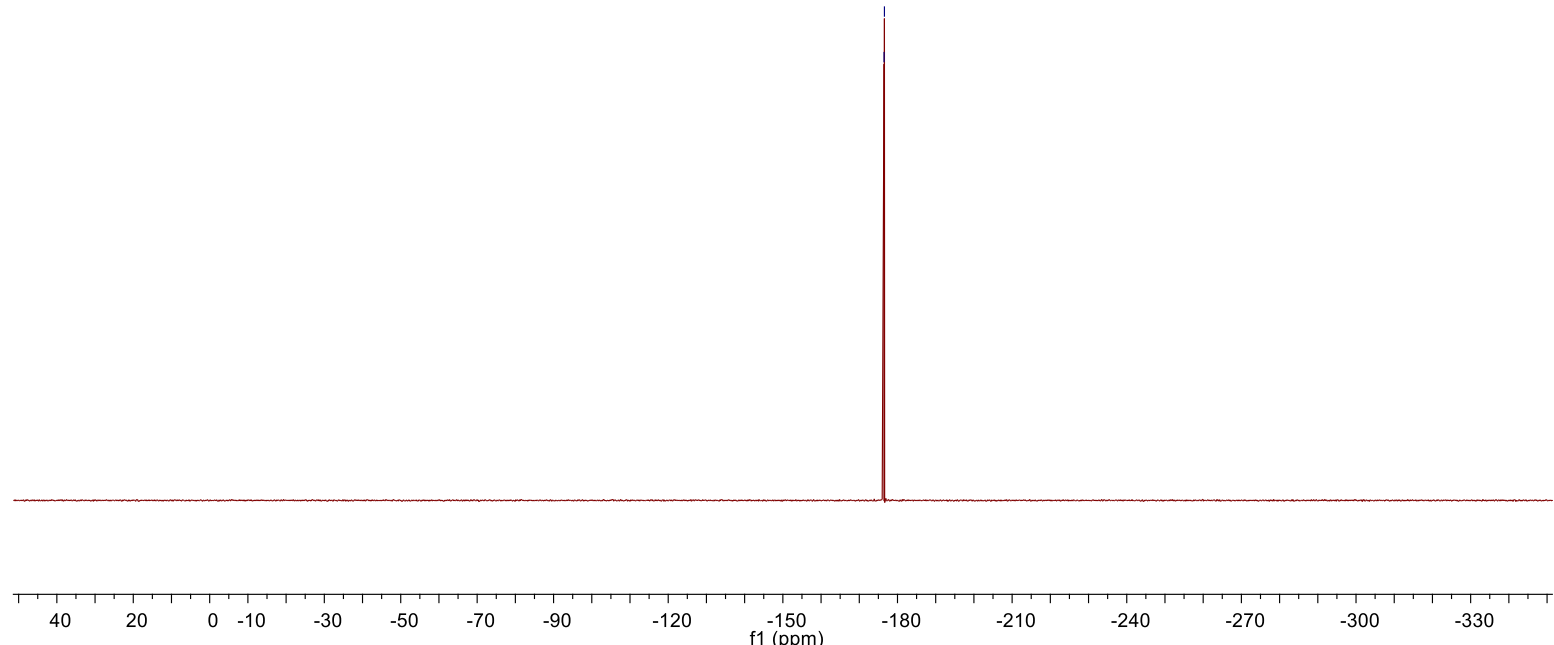

$376 \mathrm{MHz}{ }^{19} \mathrm{~F}$ NMR spectra of compound 7 in $\mathrm{CDCl}_{3}$ 


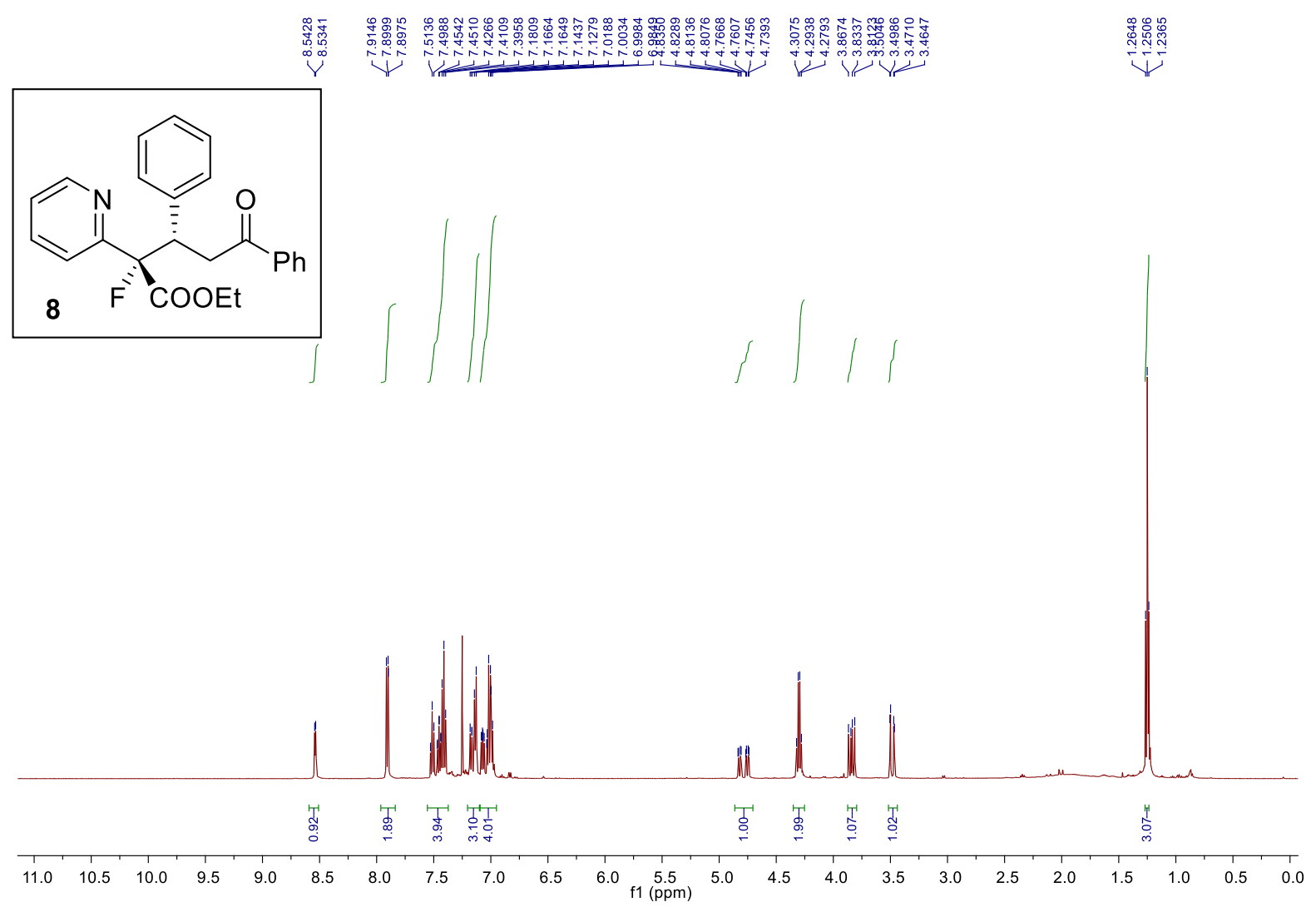

$500 \mathrm{MHz}{ }^{1} \mathrm{H}$ NMR spectra of compound 8 in $\mathrm{CDCl}_{3}$
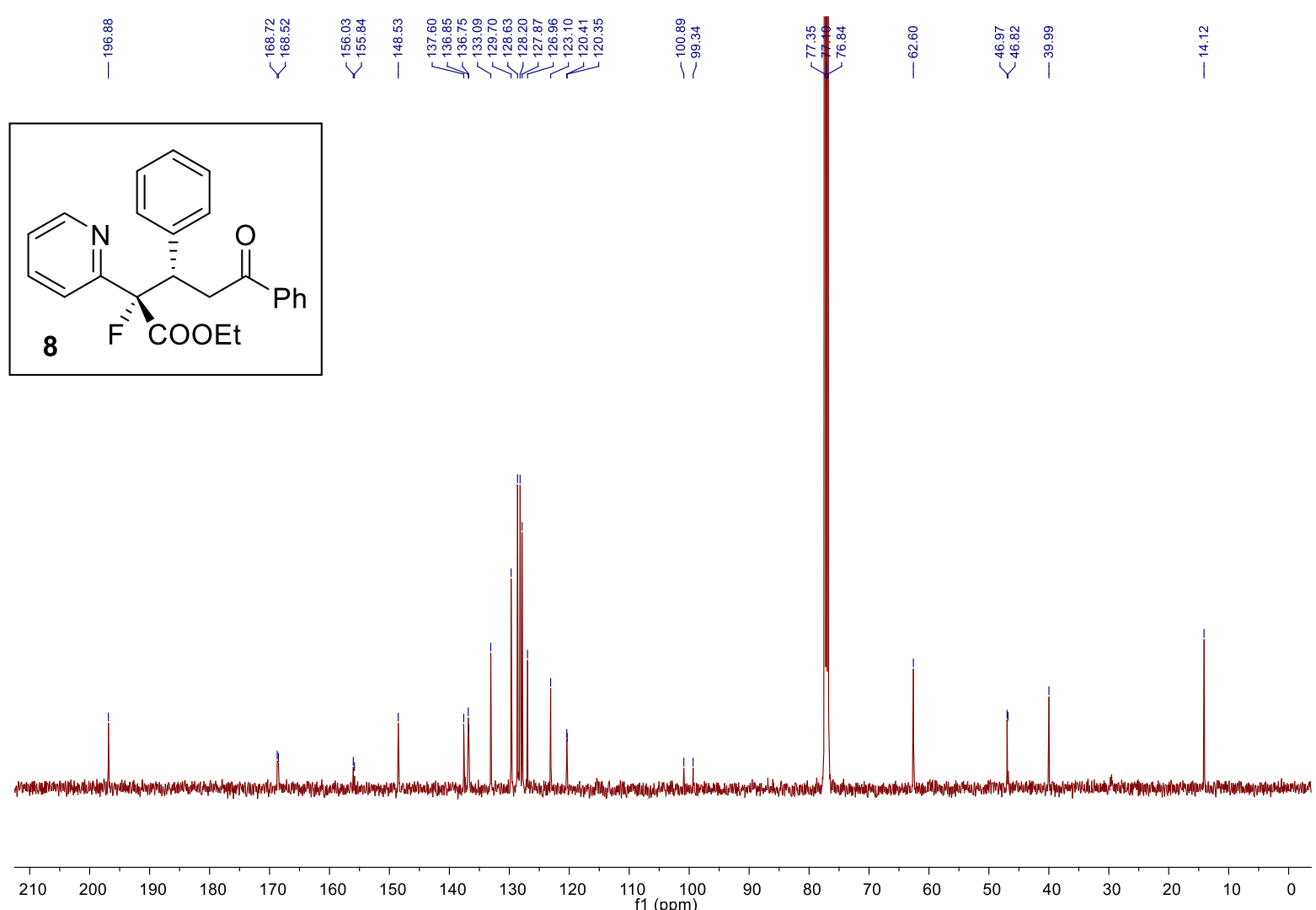

$125 \mathrm{MHz}{ }^{13} \mathrm{C}$ NMR spectra of compound 8 in $\mathrm{CDCl}_{3}$ 


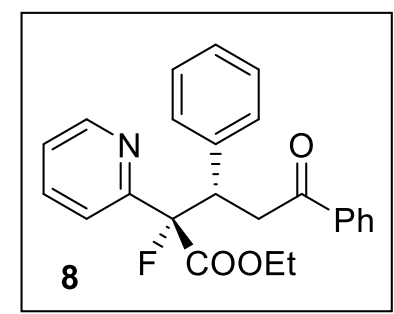

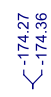

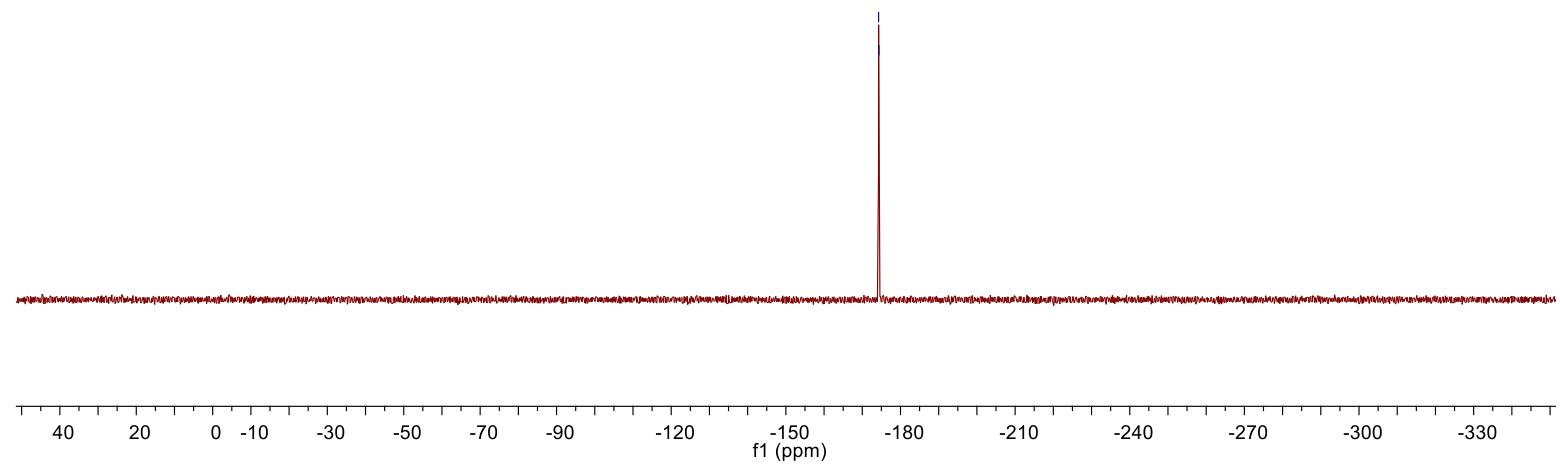

$376 \mathrm{MHz}{ }^{19} \mathrm{~F}$ NMR spectra of compound 8 in $\mathrm{CDCl}_{3}$ 


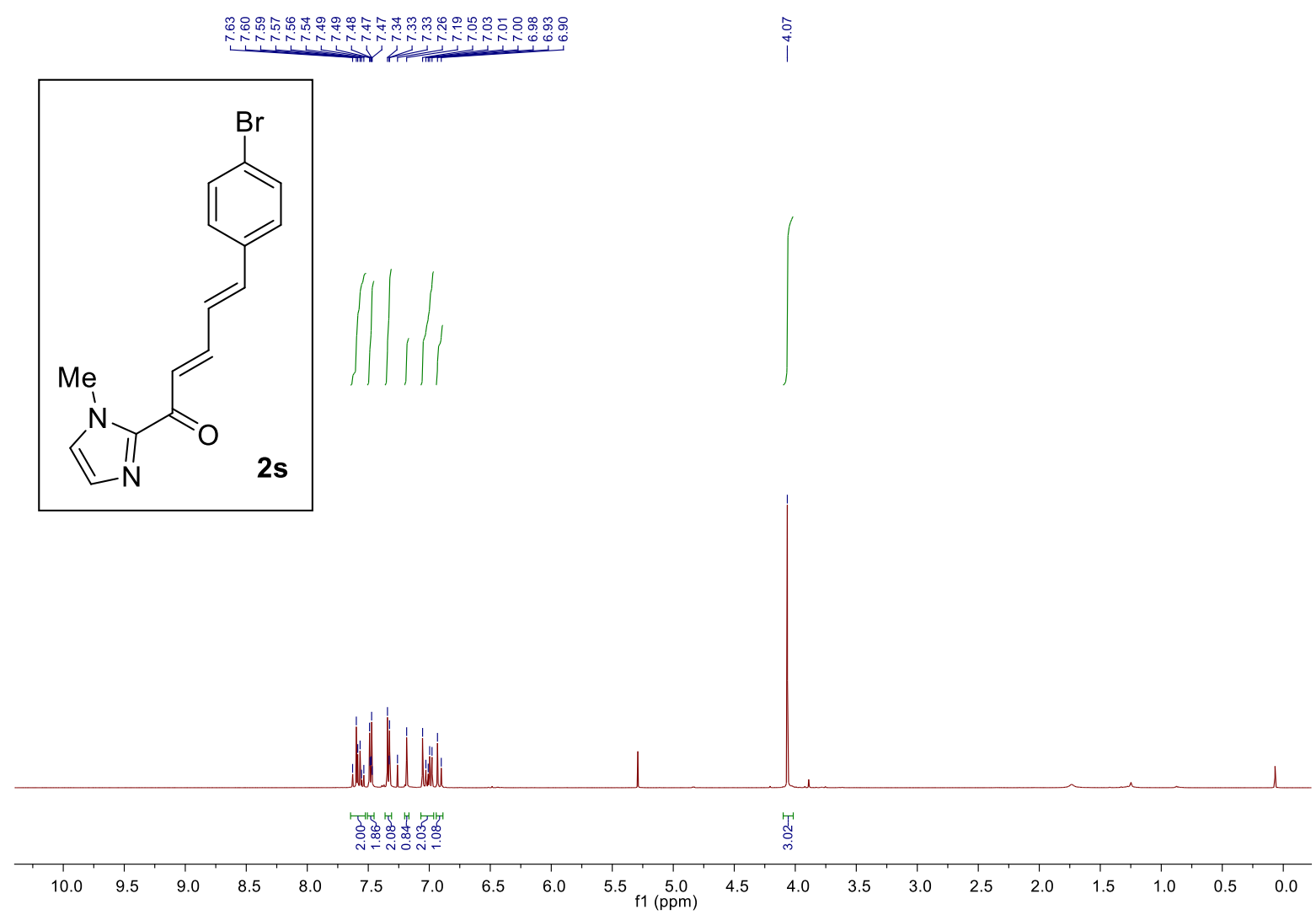

$500 \mathrm{MHz}{ }^{1} \mathrm{H}$ NMR spectra of compound $2 \mathrm{~s}$ in $\mathrm{CDCl}_{3}$

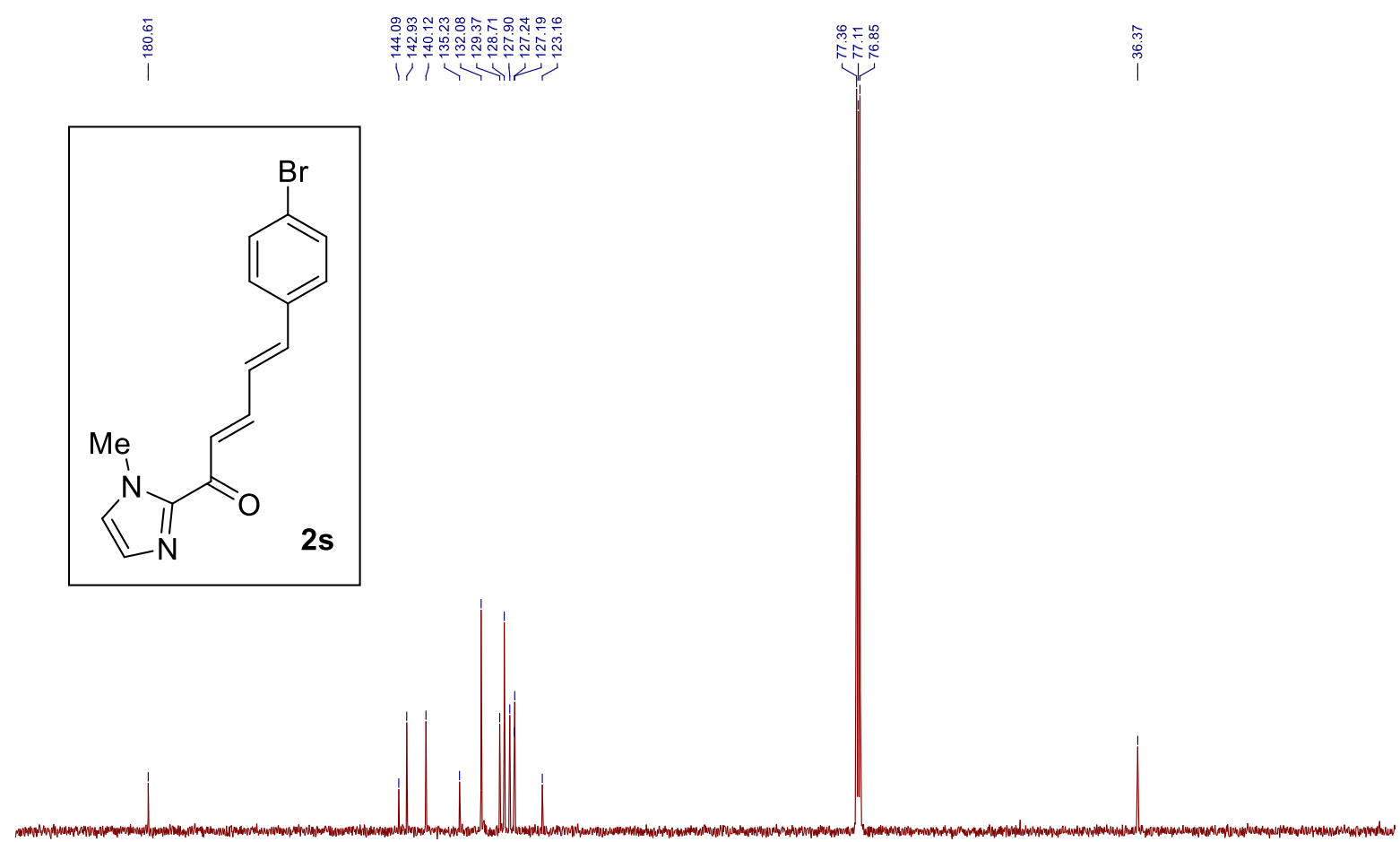

$\begin{array}{lllllllllllllllllllllll}190 & 180 & 170 & 160 & 150 & 140 & 130 & 120 & 110 & 100 & 90 & 80 & 70 & 60 & 50 & 40 & 30 & 20 & 10 & 0\end{array}$

$125 \mathrm{MHz}{ }^{13} \mathrm{C}$ NMR spectra of compound $2 \mathrm{~s}$ in $\mathrm{CDCl}_{3}$ 


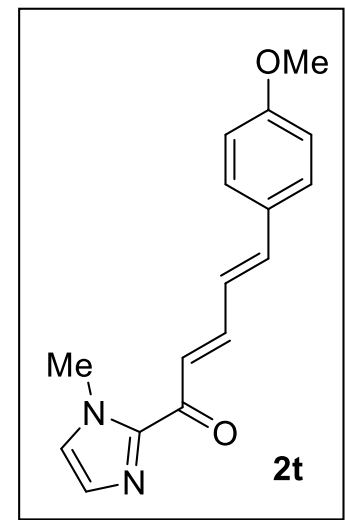

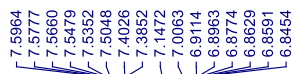
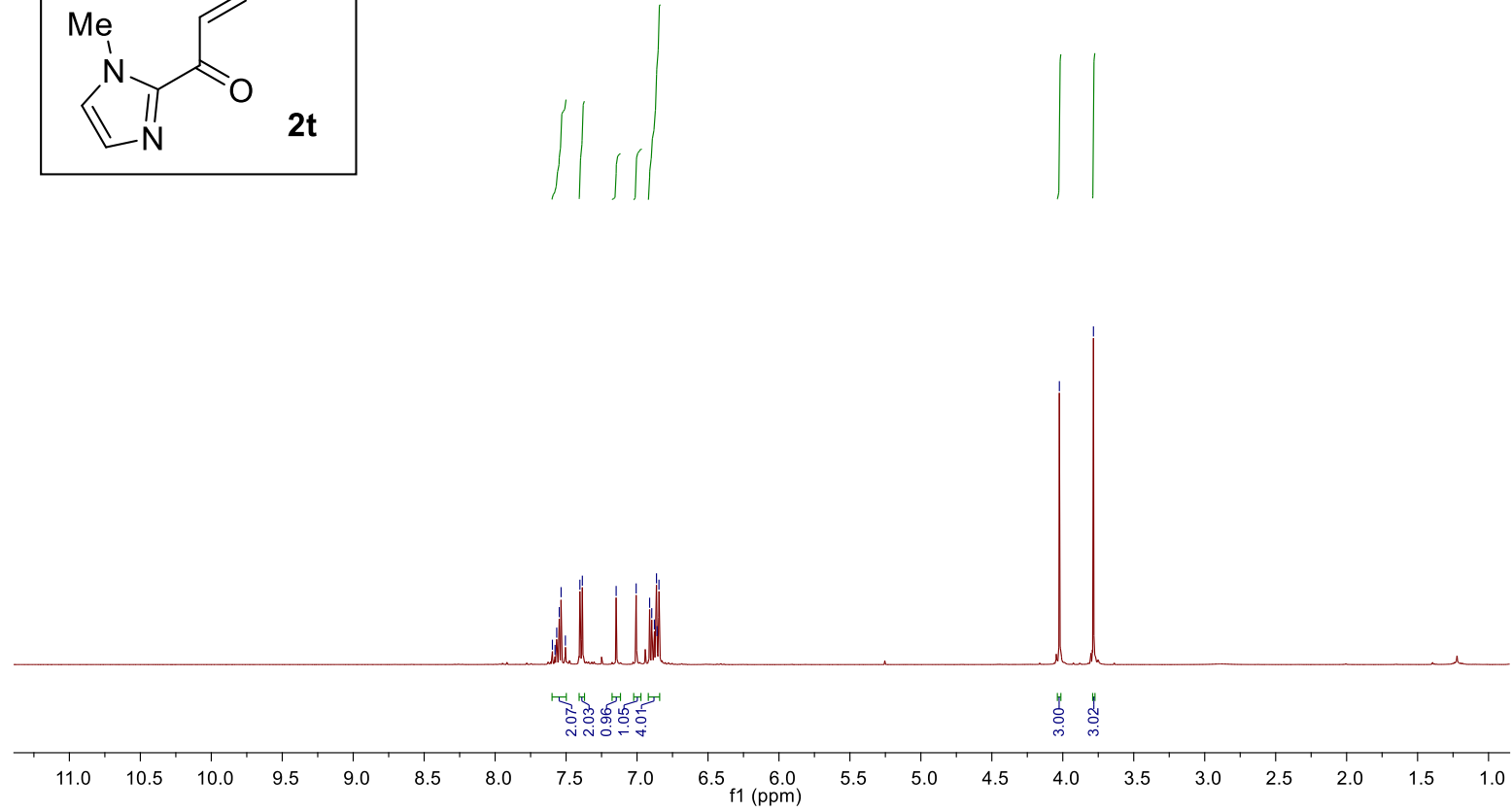

$500 \mathrm{MHz}{ }^{1} \mathrm{H}$ NMR spectra of compound $2 \mathrm{t}$ in $\mathrm{CDCl}_{3}$

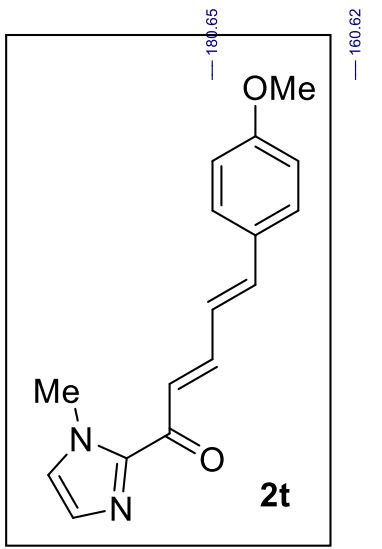

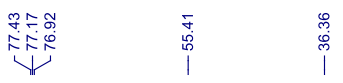

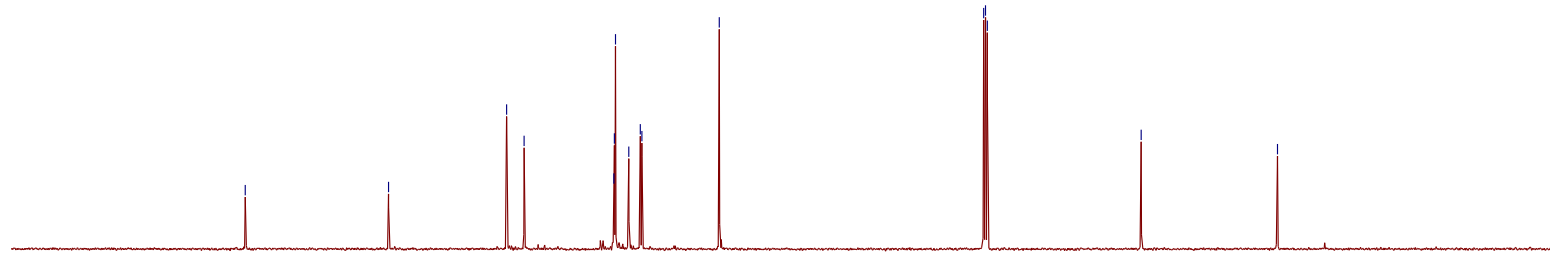

$\begin{array}{llllllllllllllllllllll}210 & 200 & 190 & 180 & 170 & 160 & 150 & 140 & 130 & 120 & 110 & 100 & 90 & 80 & 70 & 60 & 50 & 40 & 30 & 20 & 10 & 0\end{array}$

$125 \mathrm{MHz}{ }^{13} \mathrm{C}$ NMR spectra of compound $2 \mathrm{t}$ in $\mathrm{CDCl}_{3}$ 
HPLC spectra of Products 3, 4, and 5 


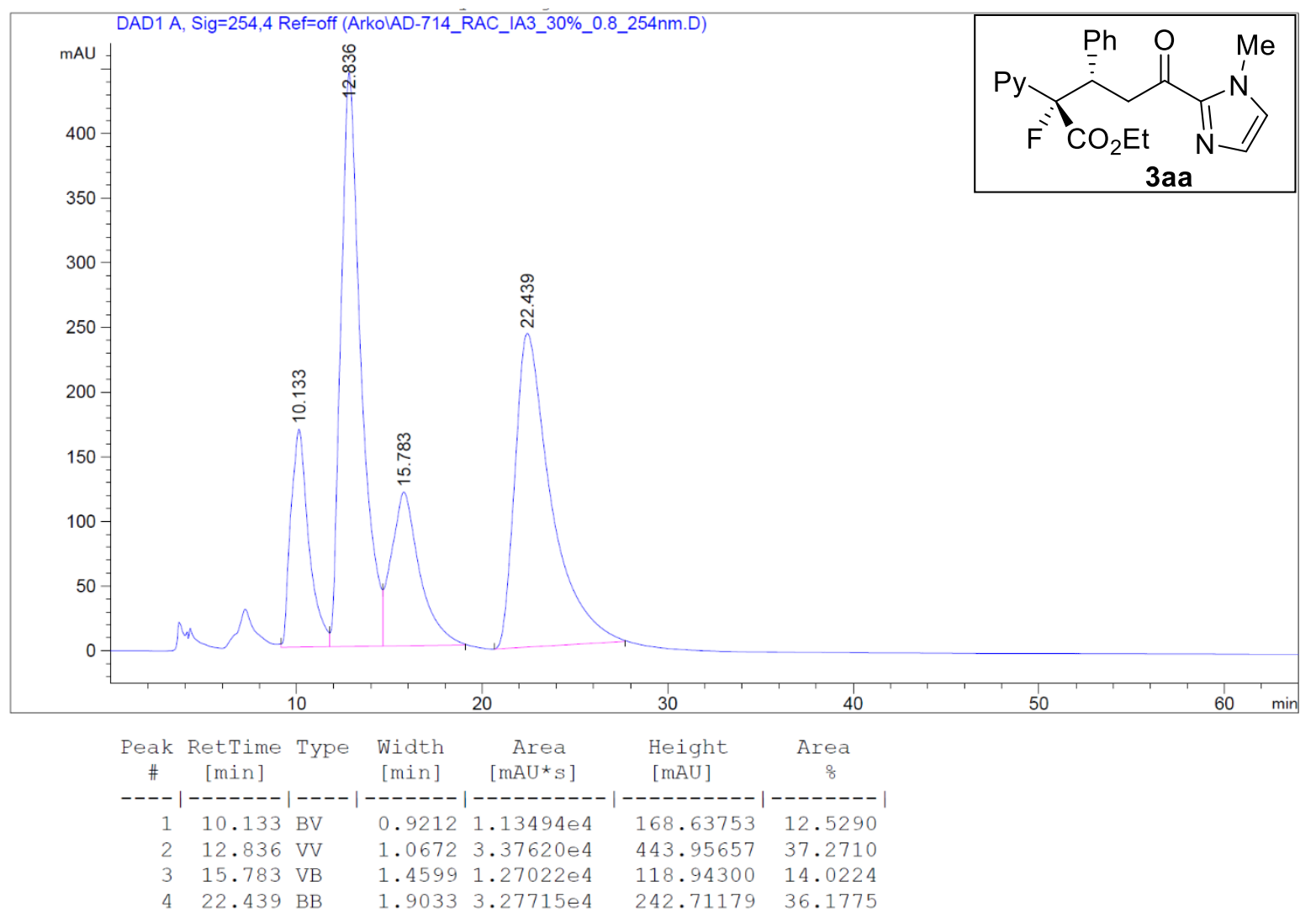

HPLC graph of racemic 3aa

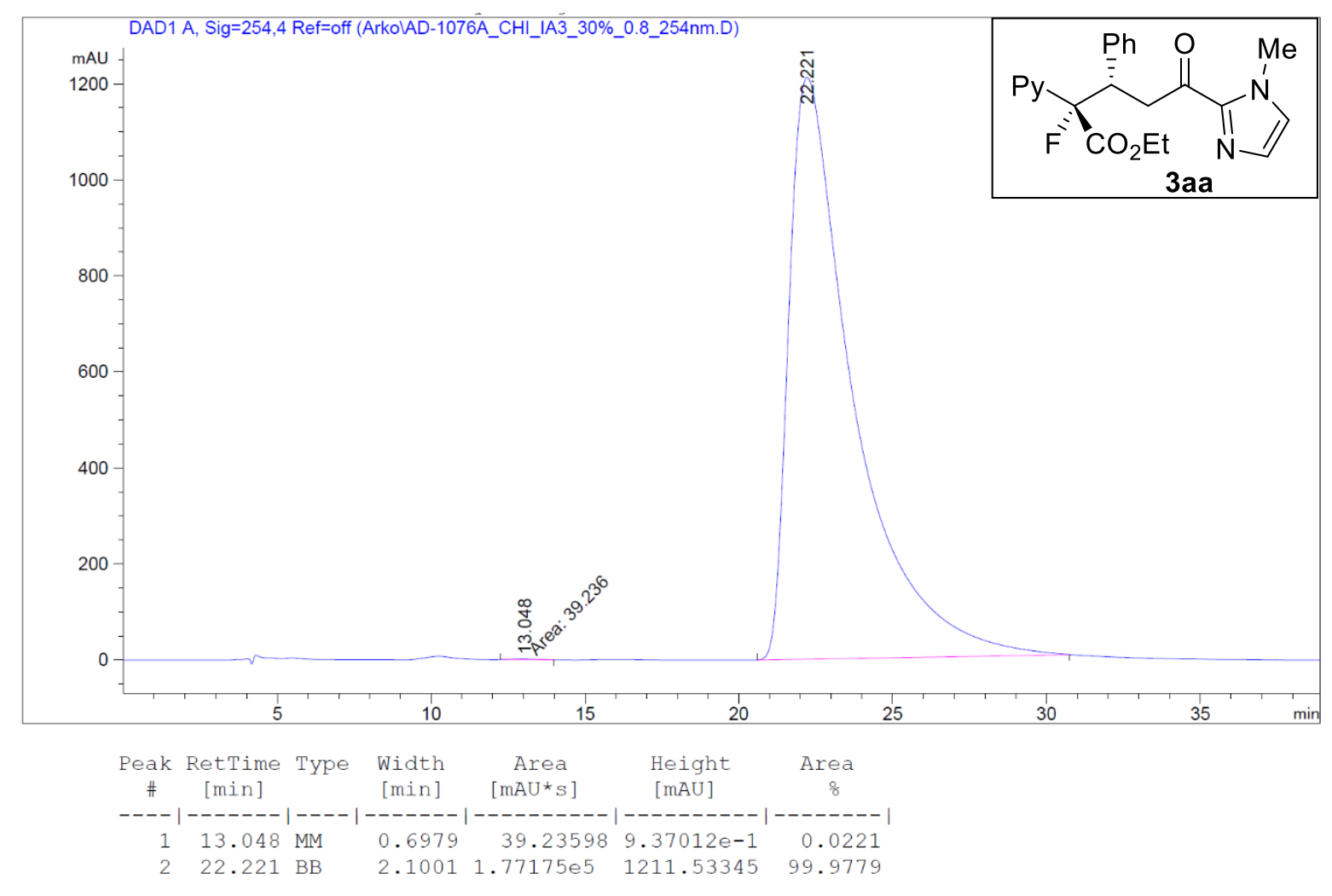

HPLC graph of chiral 3aa 


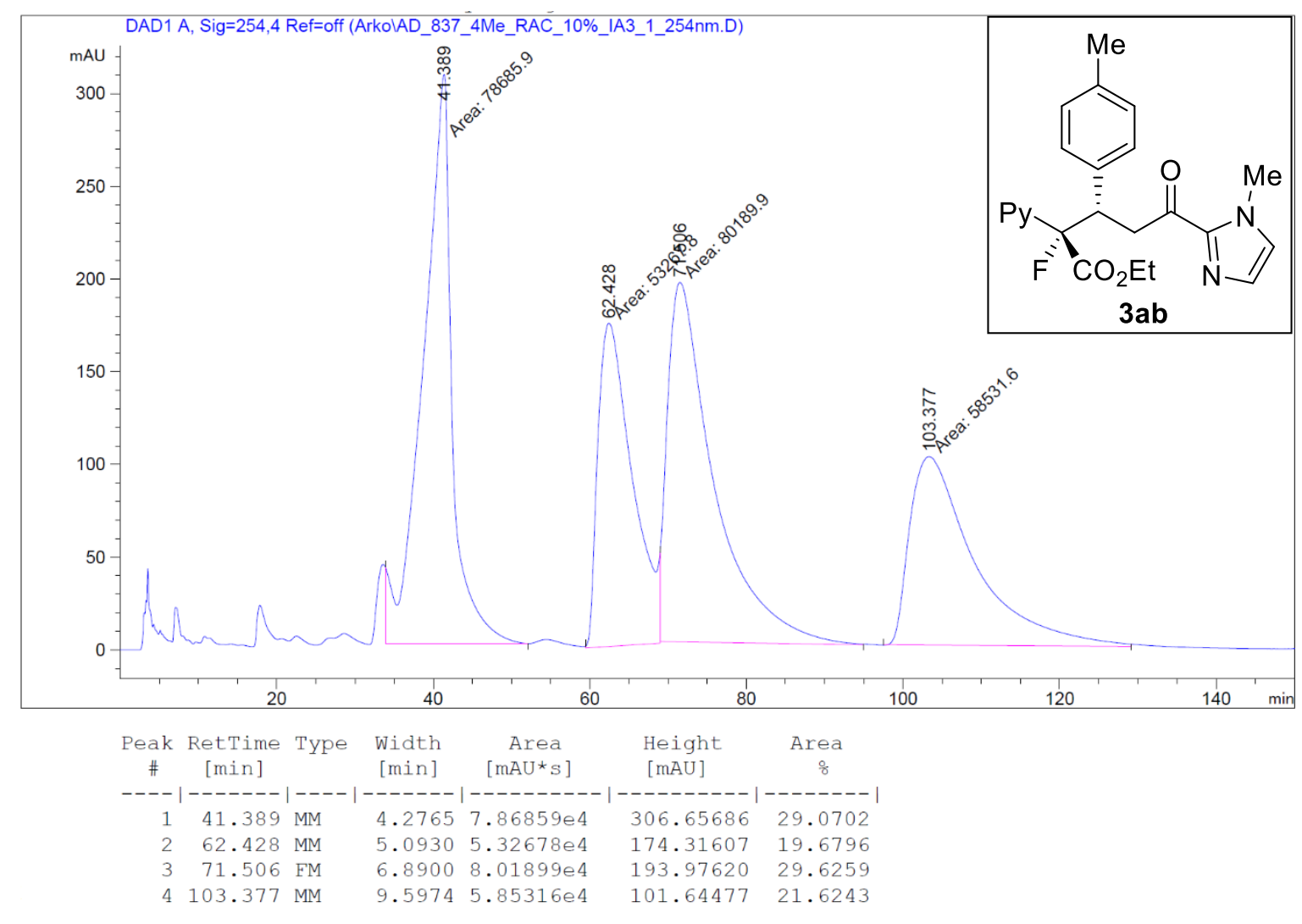

HPLC graph of racemic $3 a b$

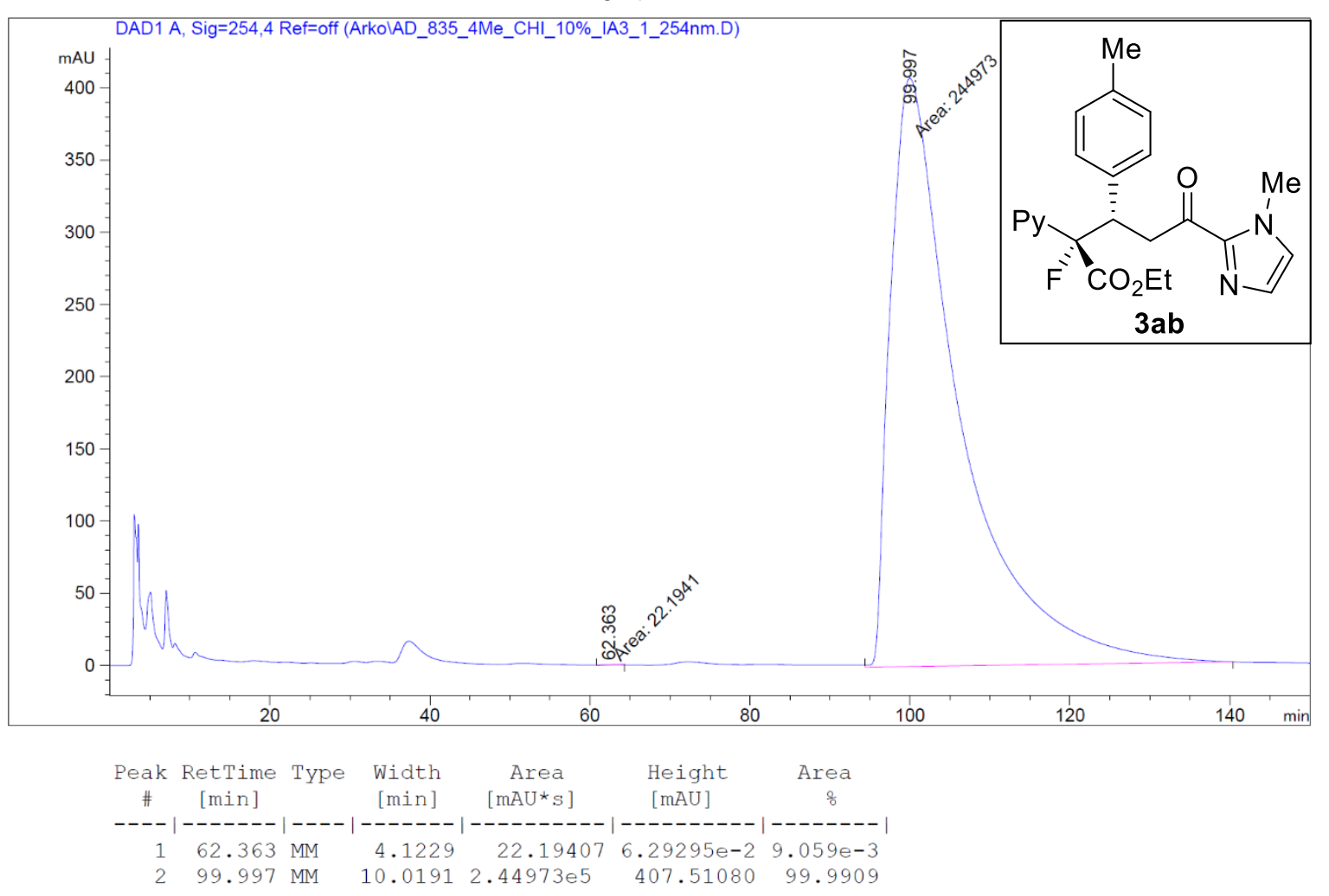

HPLC graph of chiral 3ab 


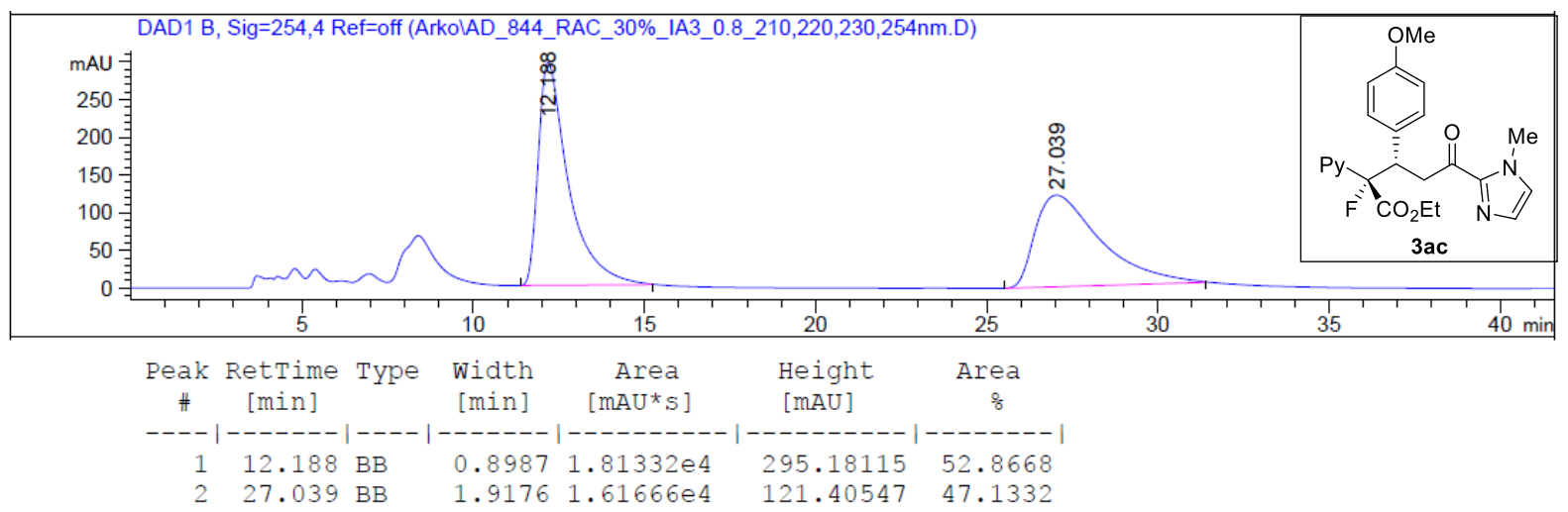

HPLC graph of racemic 3ac

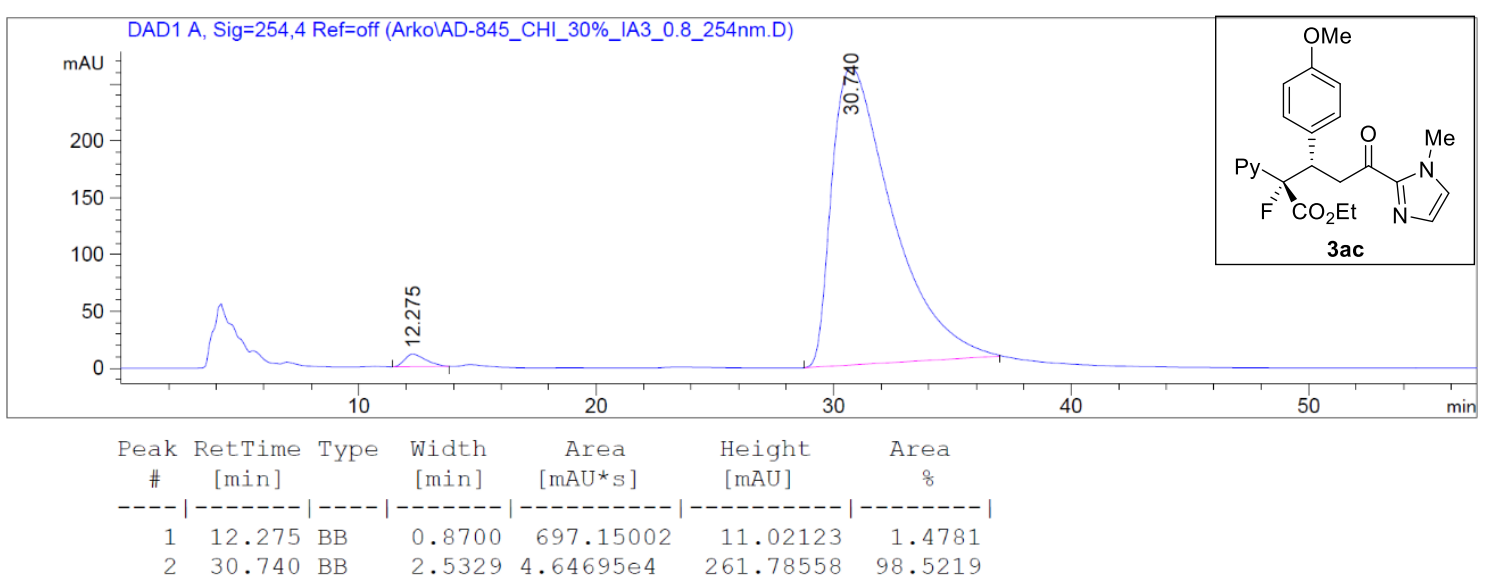

HPLC graph of chiral 3ac 


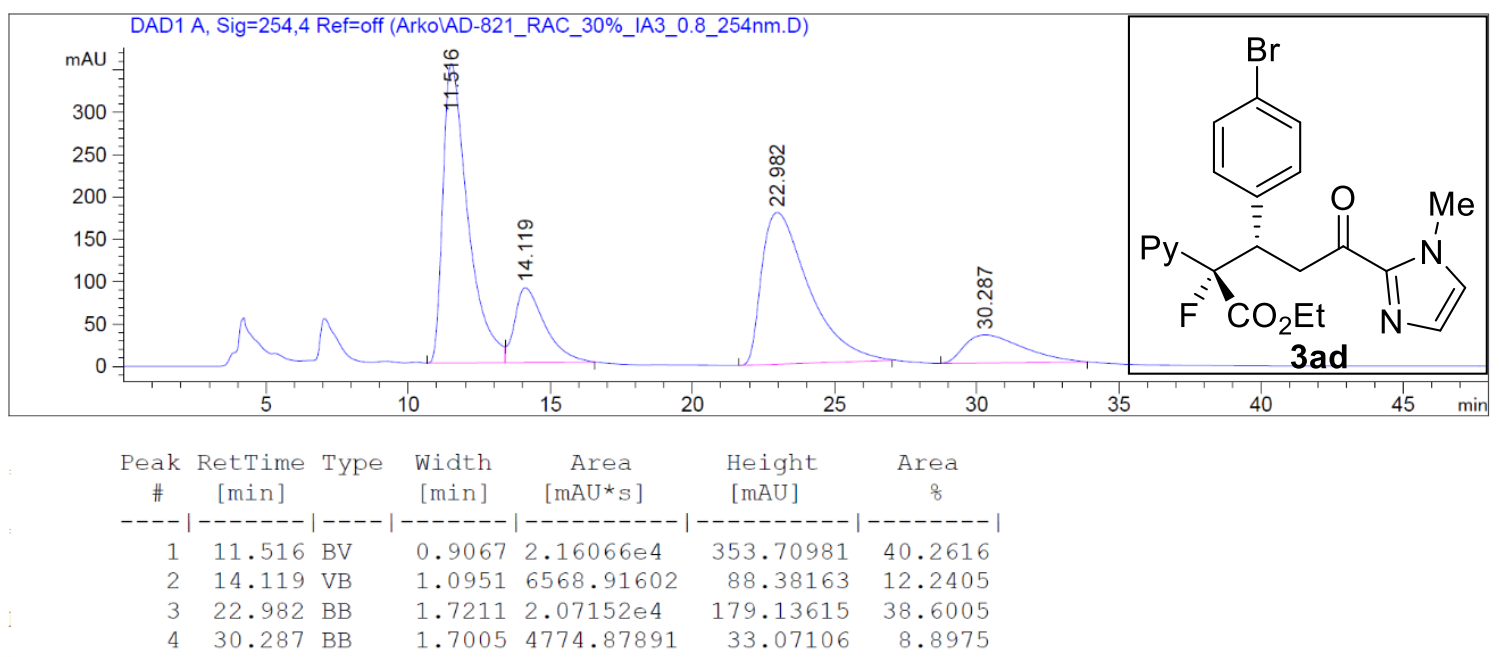

HPLC graph of racemic $3 \mathrm{ad}$

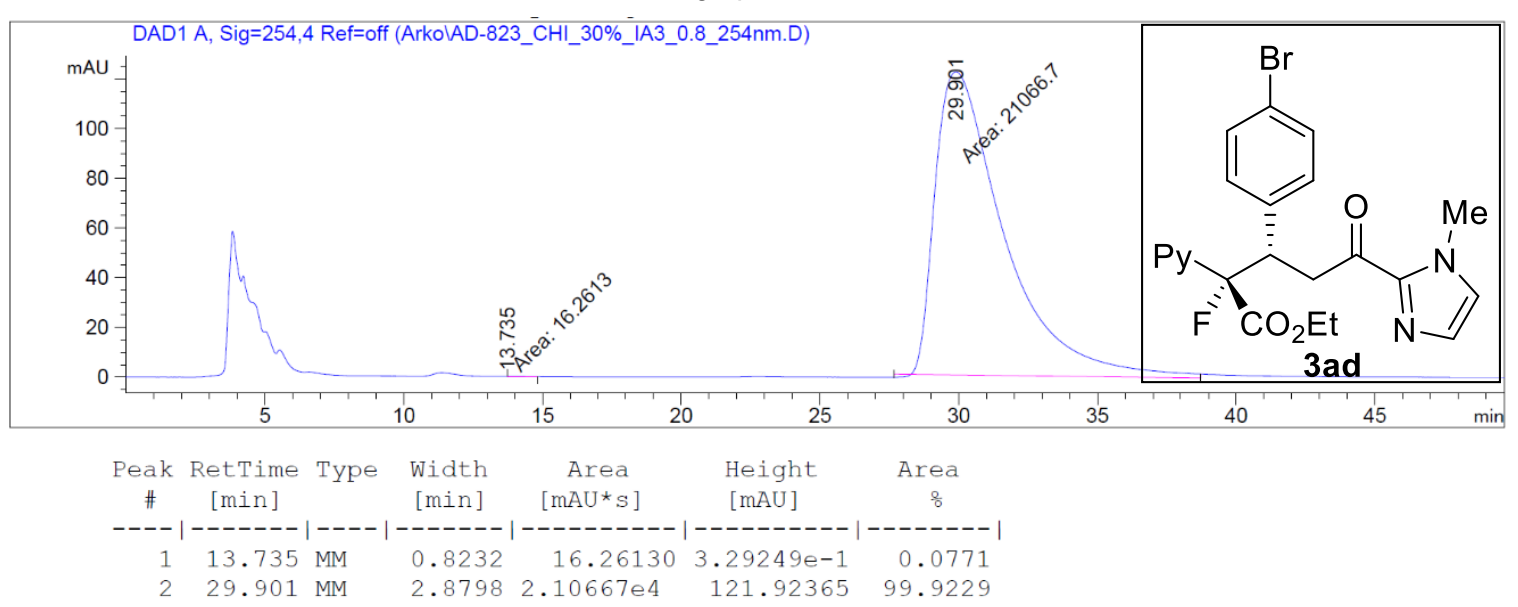

HPLC graph of chiral 3ad

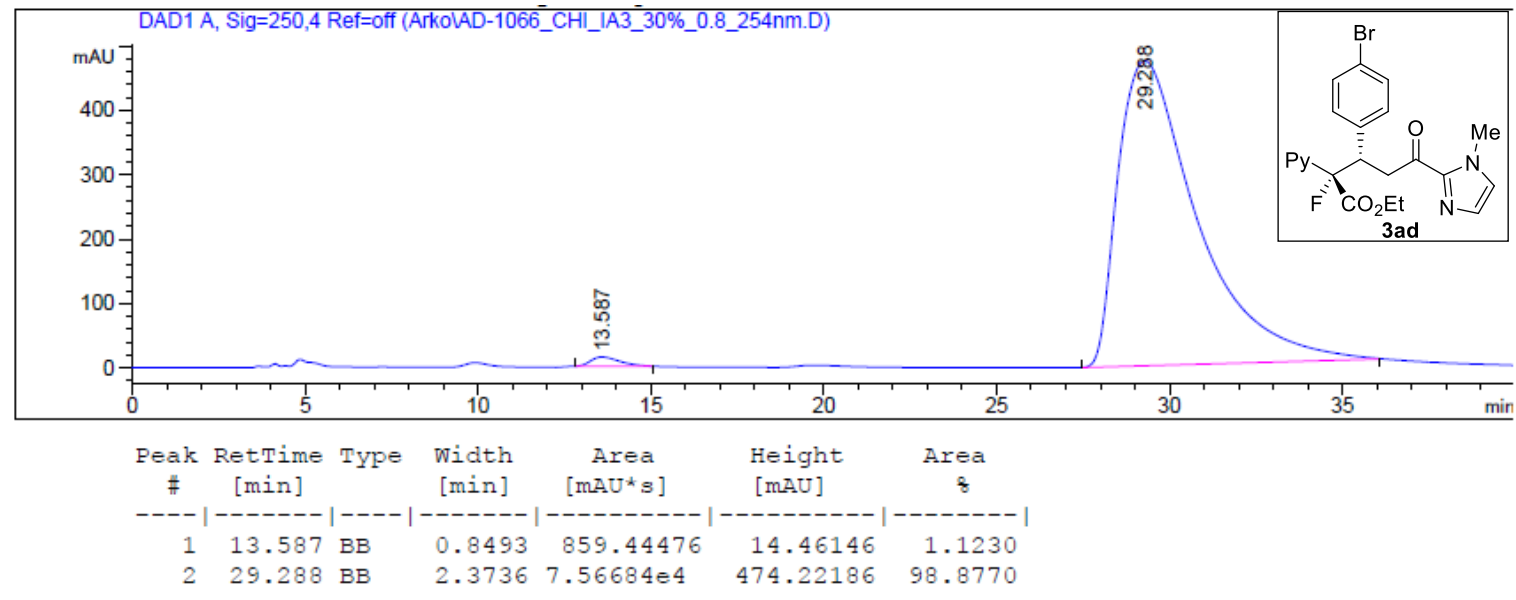

HPLC graph of chiral large-scale reaction (3ad) 


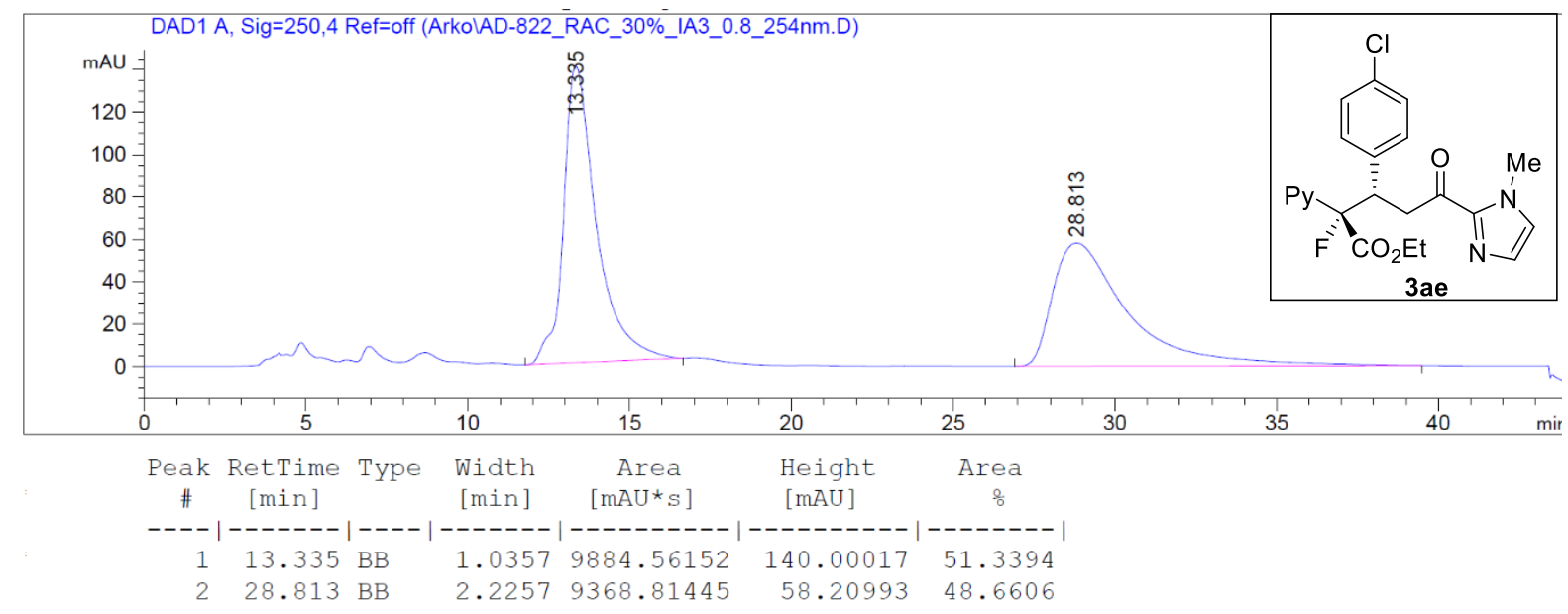

\section{HPLC graph of racemic $3 a e$}

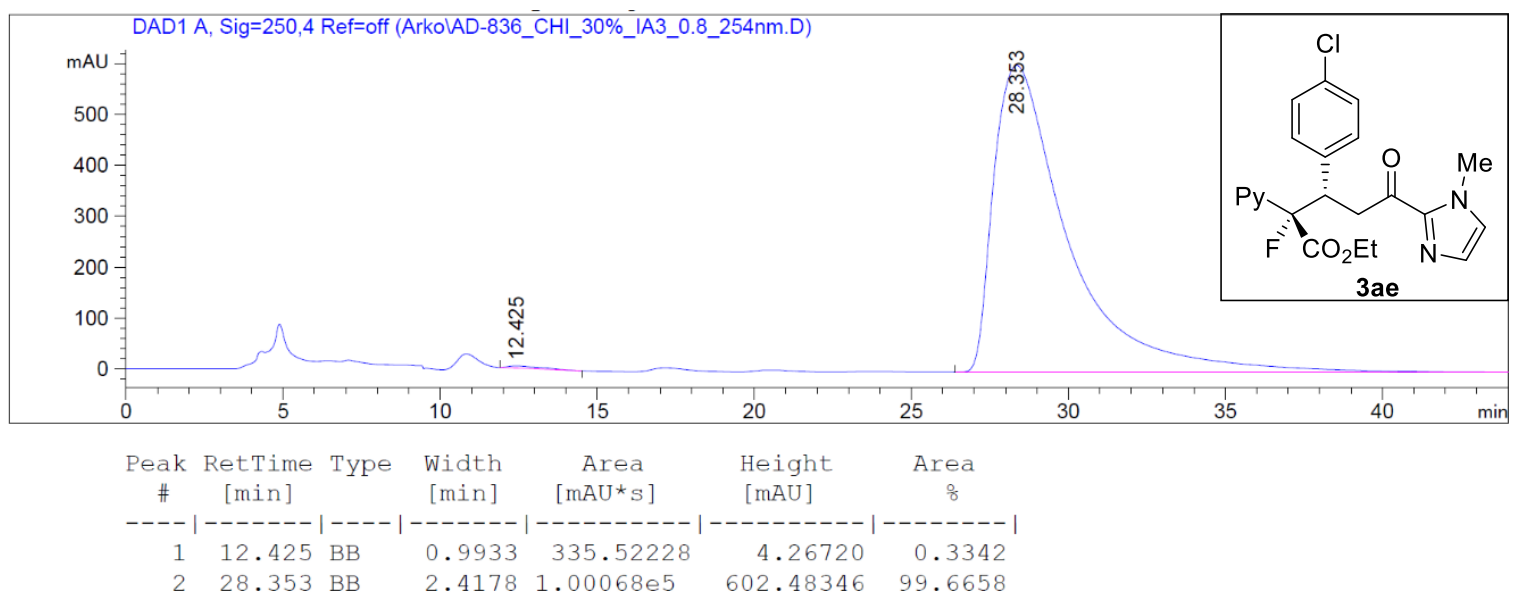

HPLC graph of chiral 3ae 


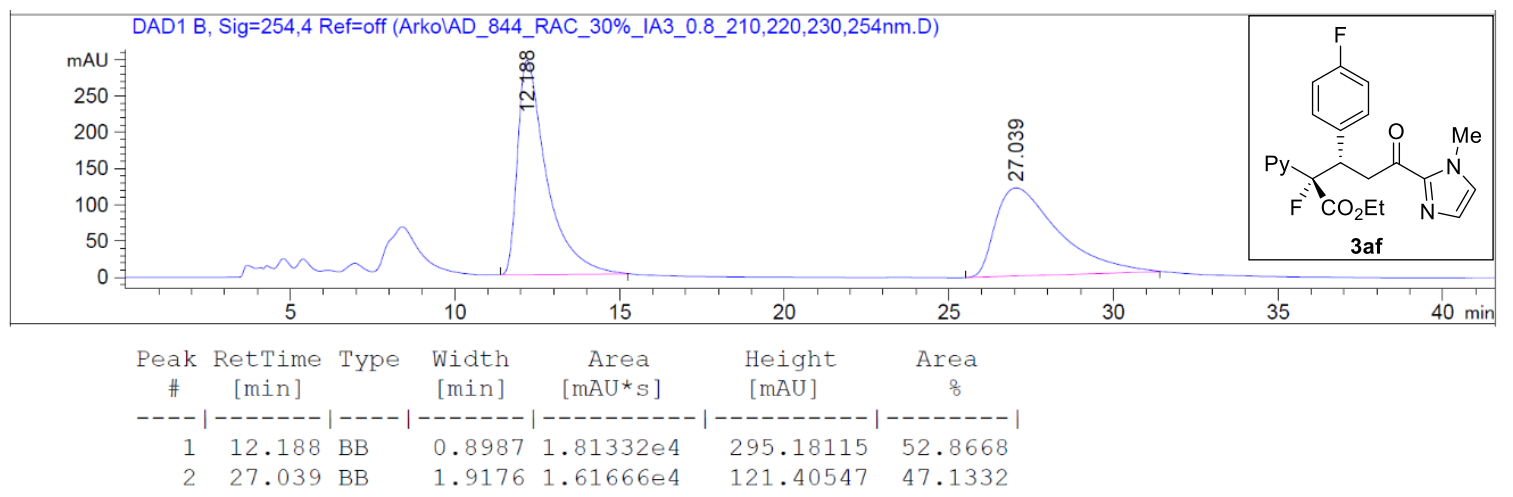

HPLC graph of racemic 3af

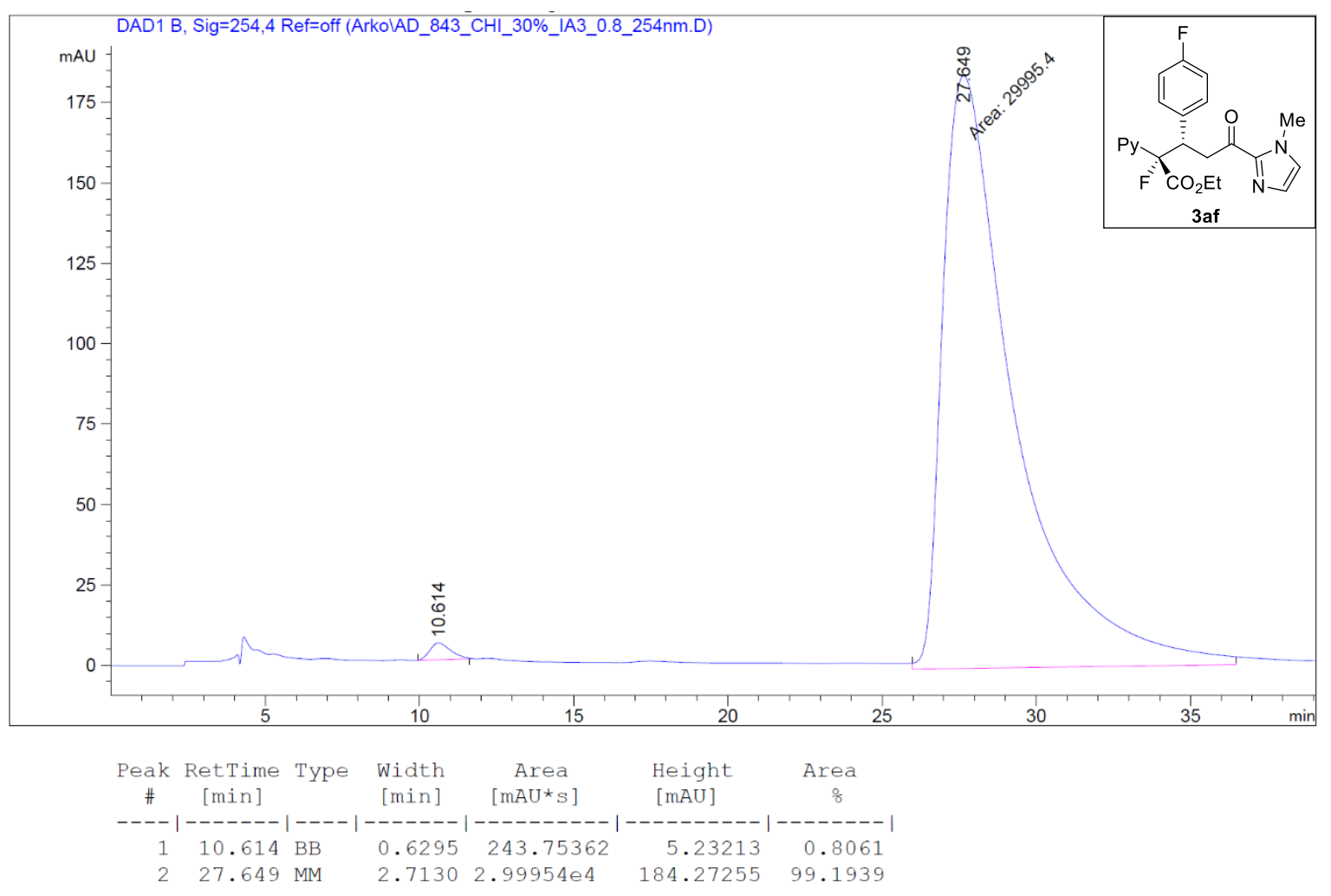

HPLC graph of chiral 3af 


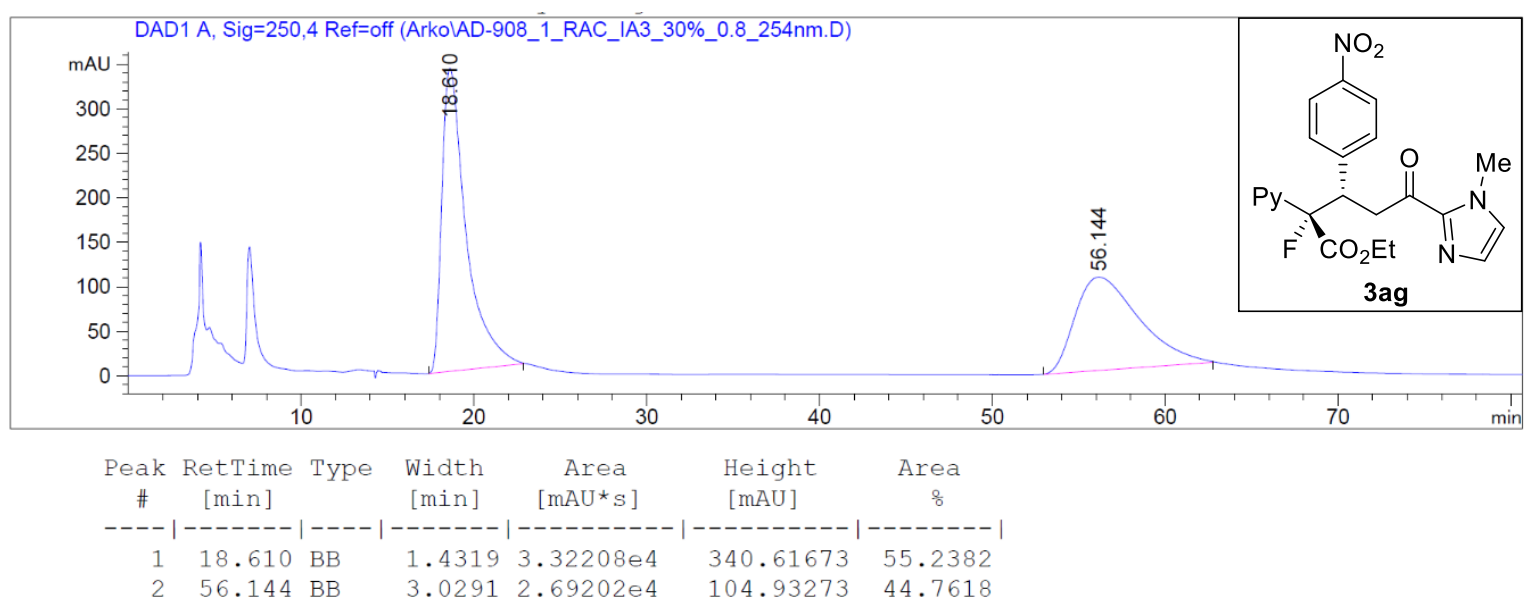

HPLC graph of racemic 3ag

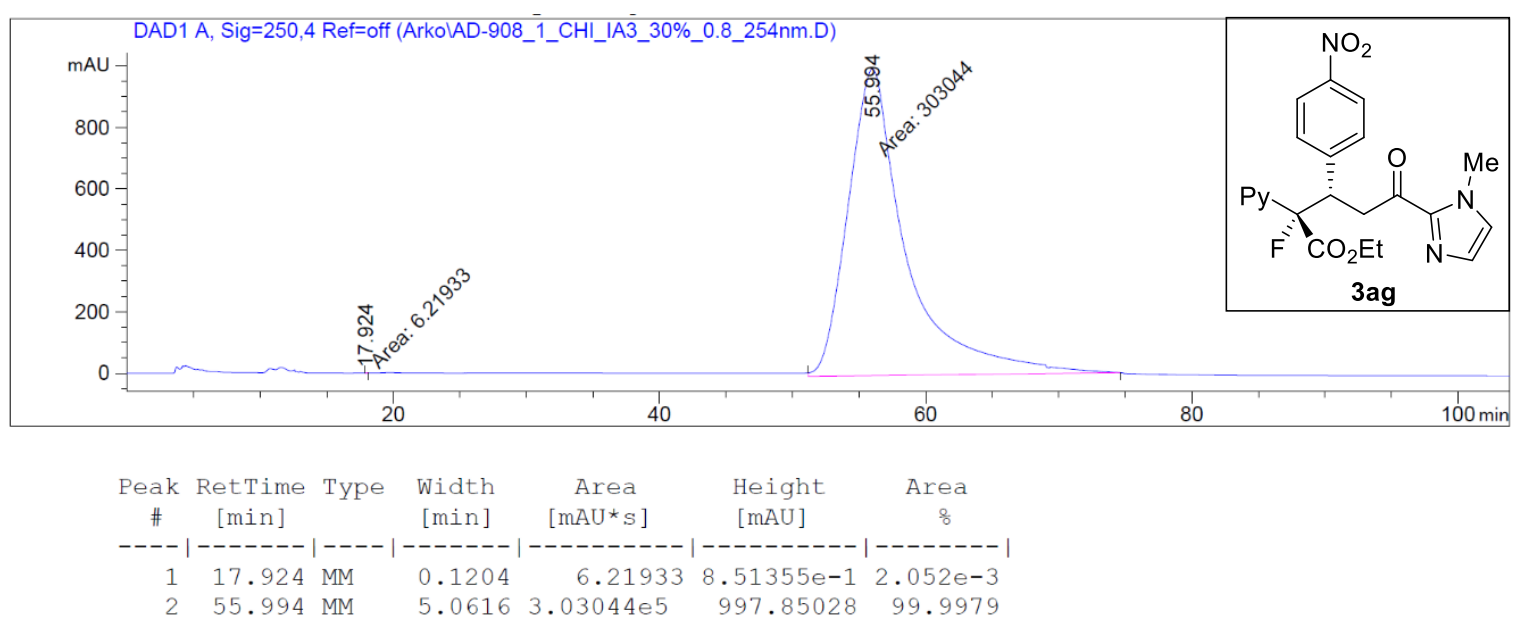

HPLC graph of chiral 3ag 


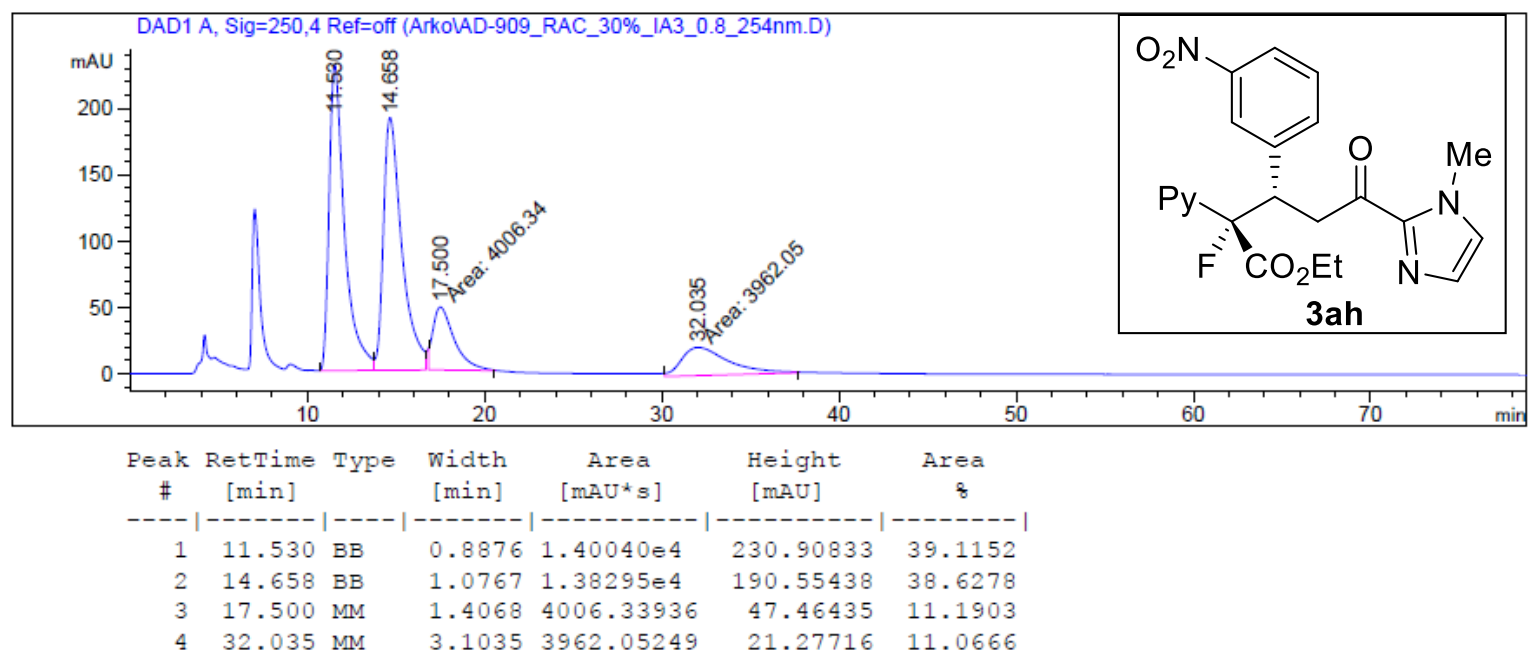

HPLC graph of racemic $3 a h$

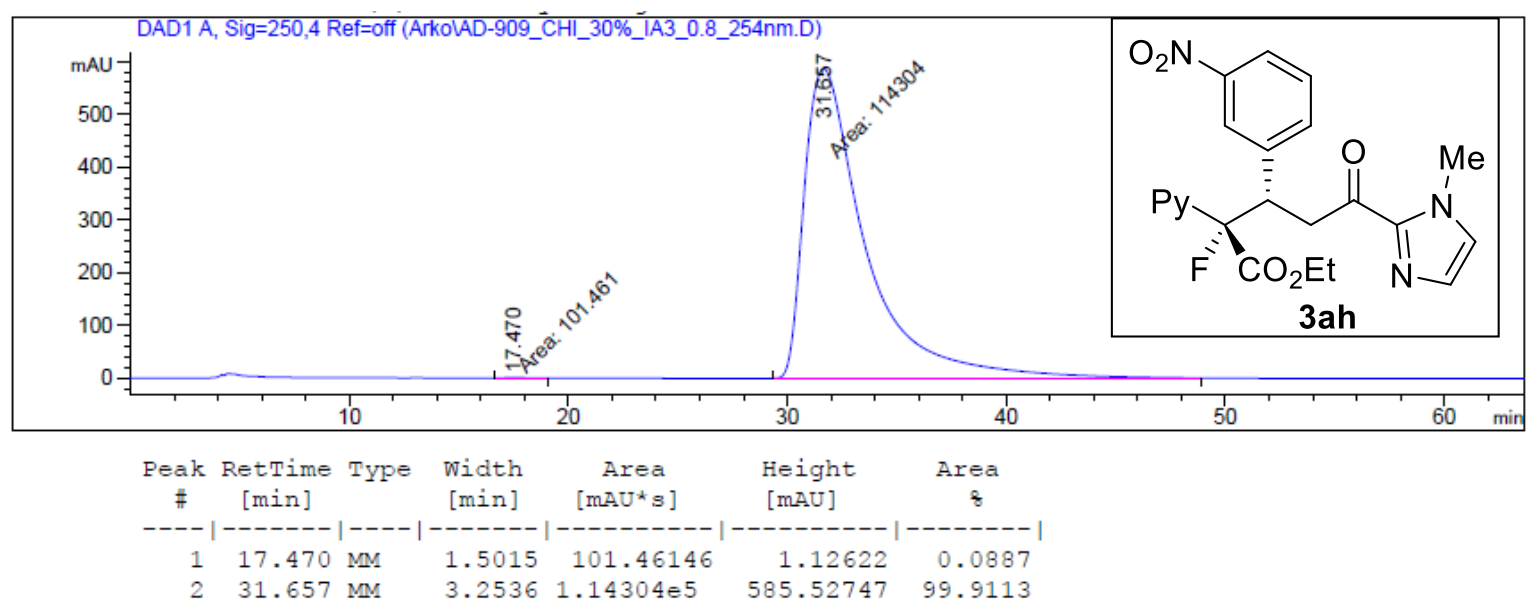

HPLC graph of chiral 3ah 


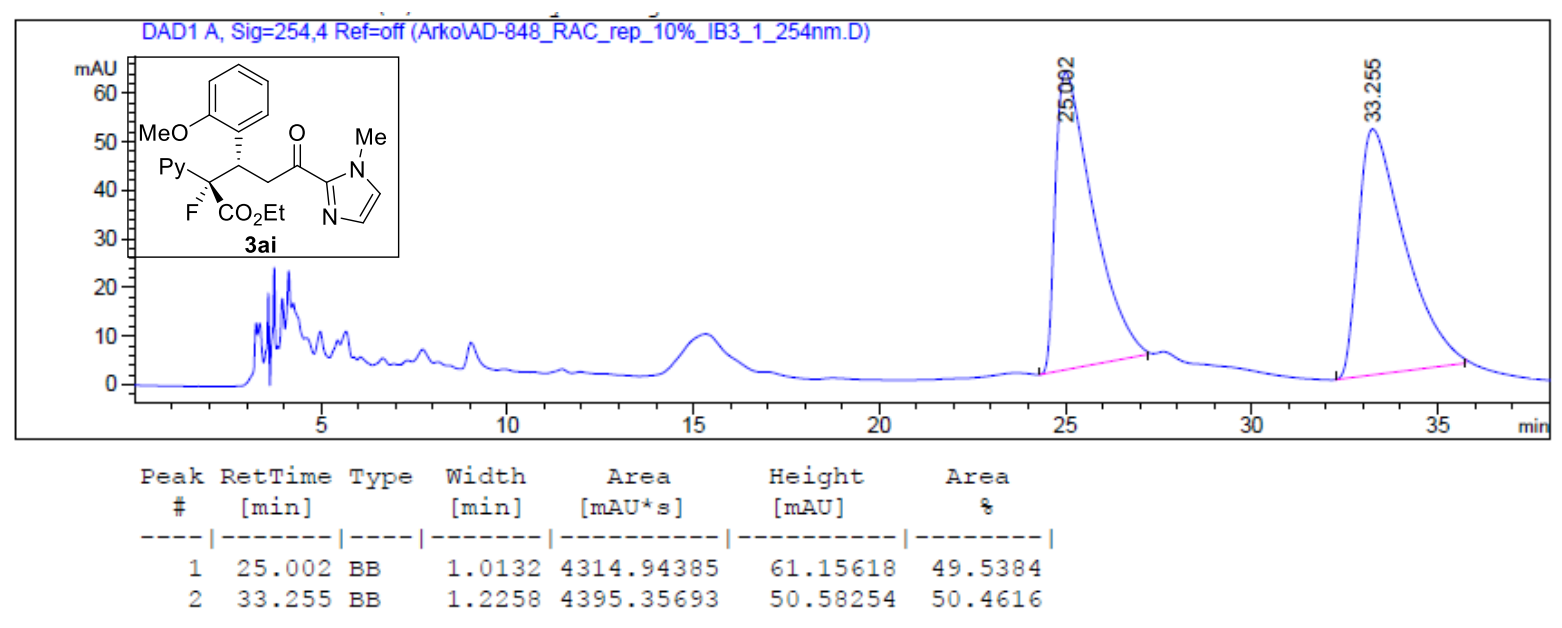

HPLC graph of racemic 3ai

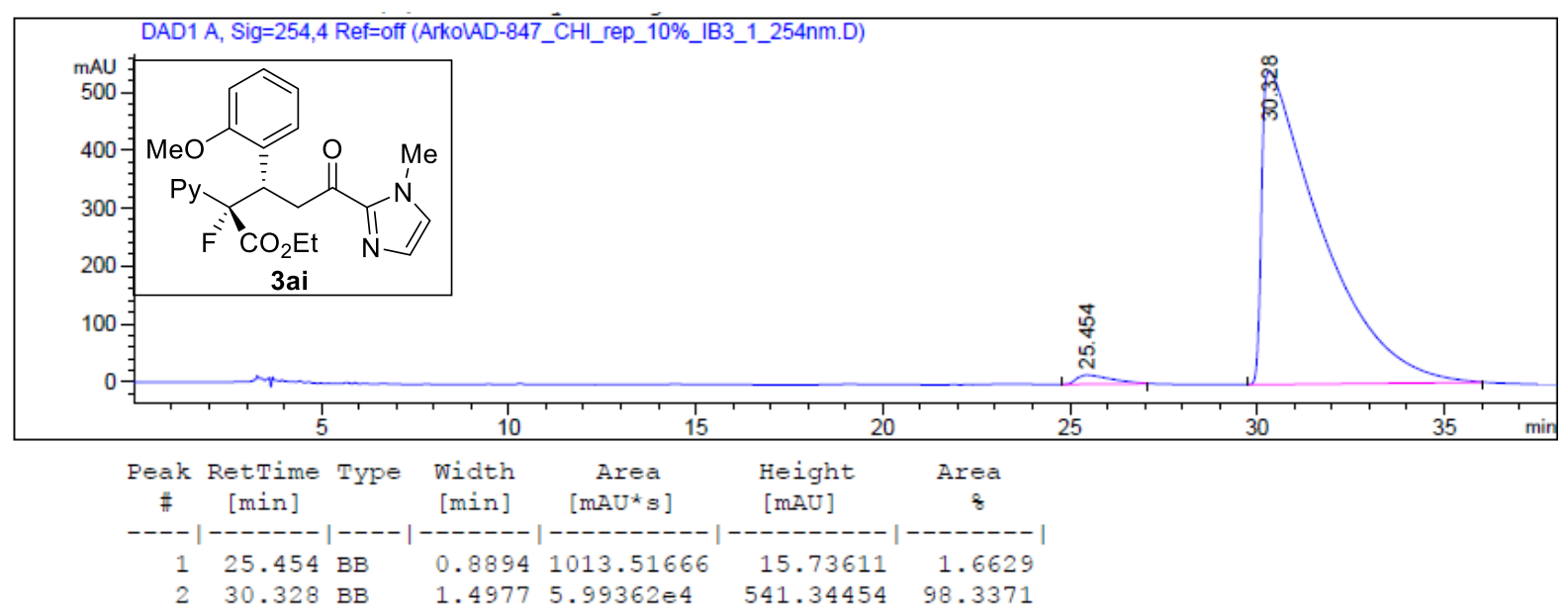

HPLC graph of chiral 3ai 


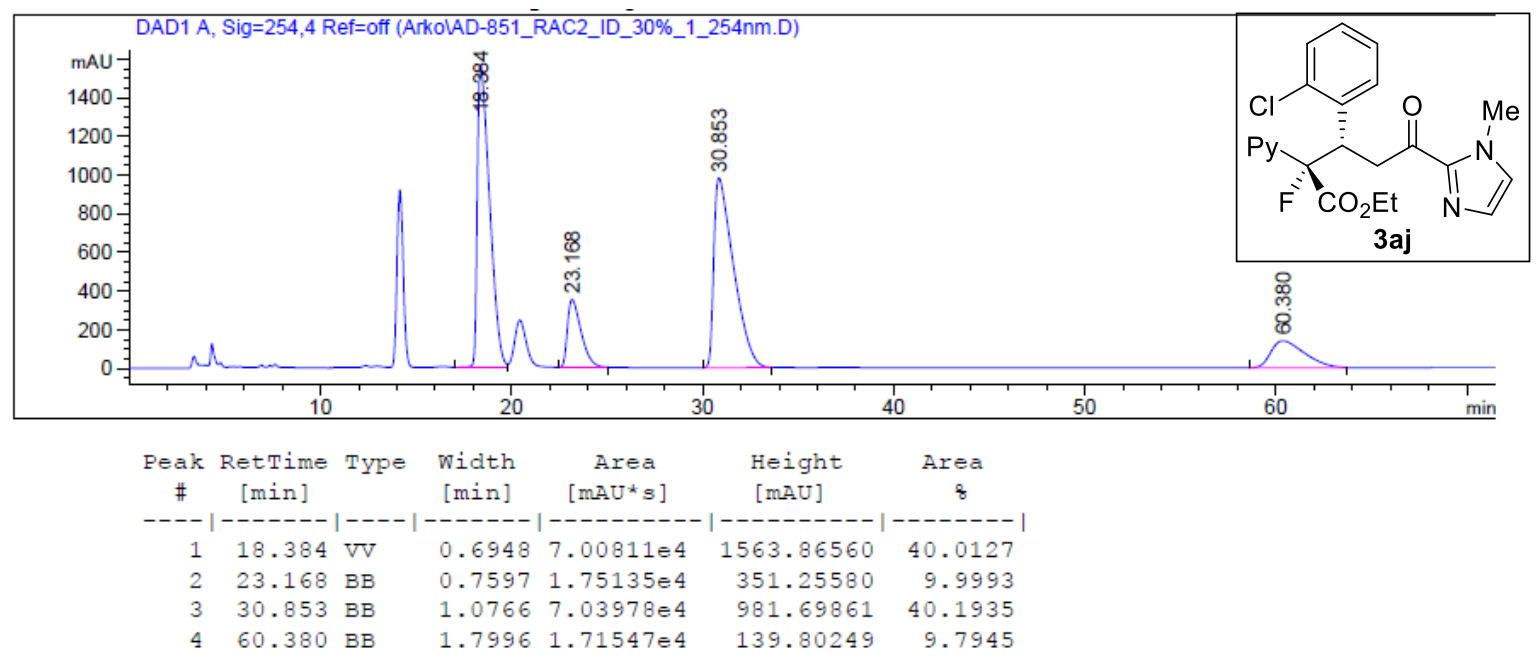

HPLC graph of racemic 3aj

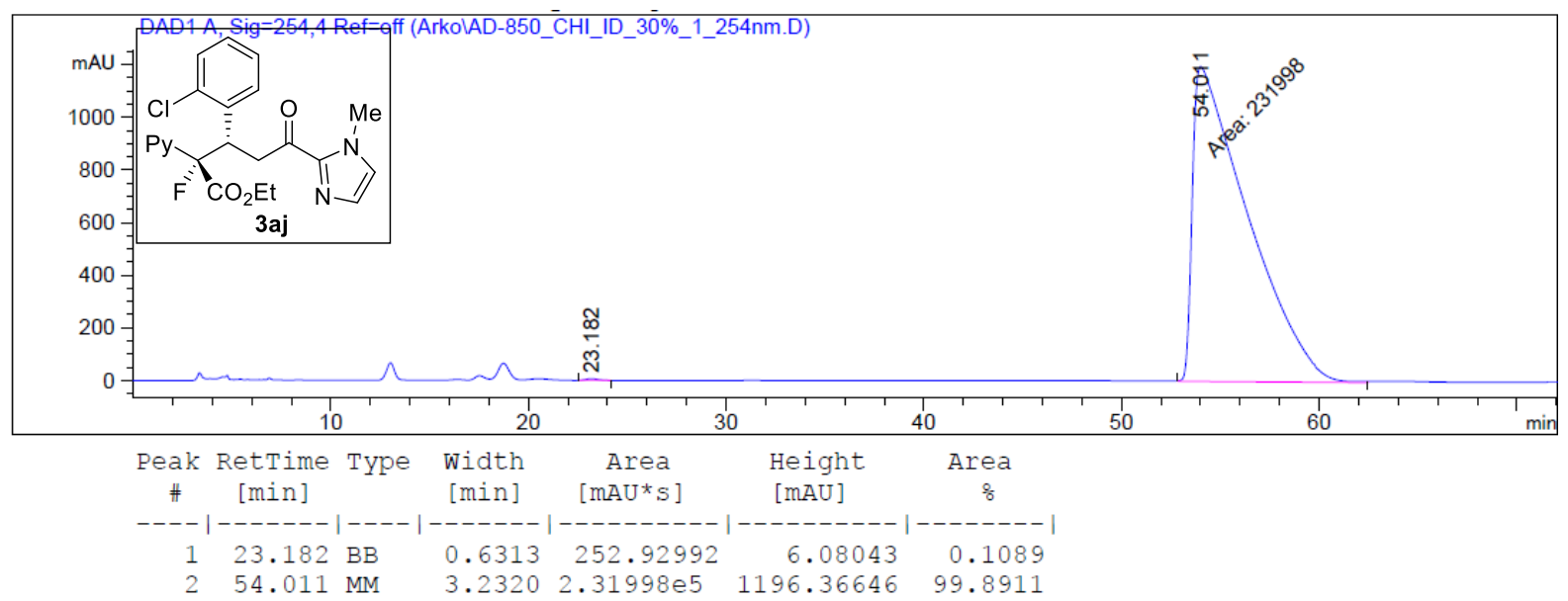

HPLC graph of chiral 3aj 


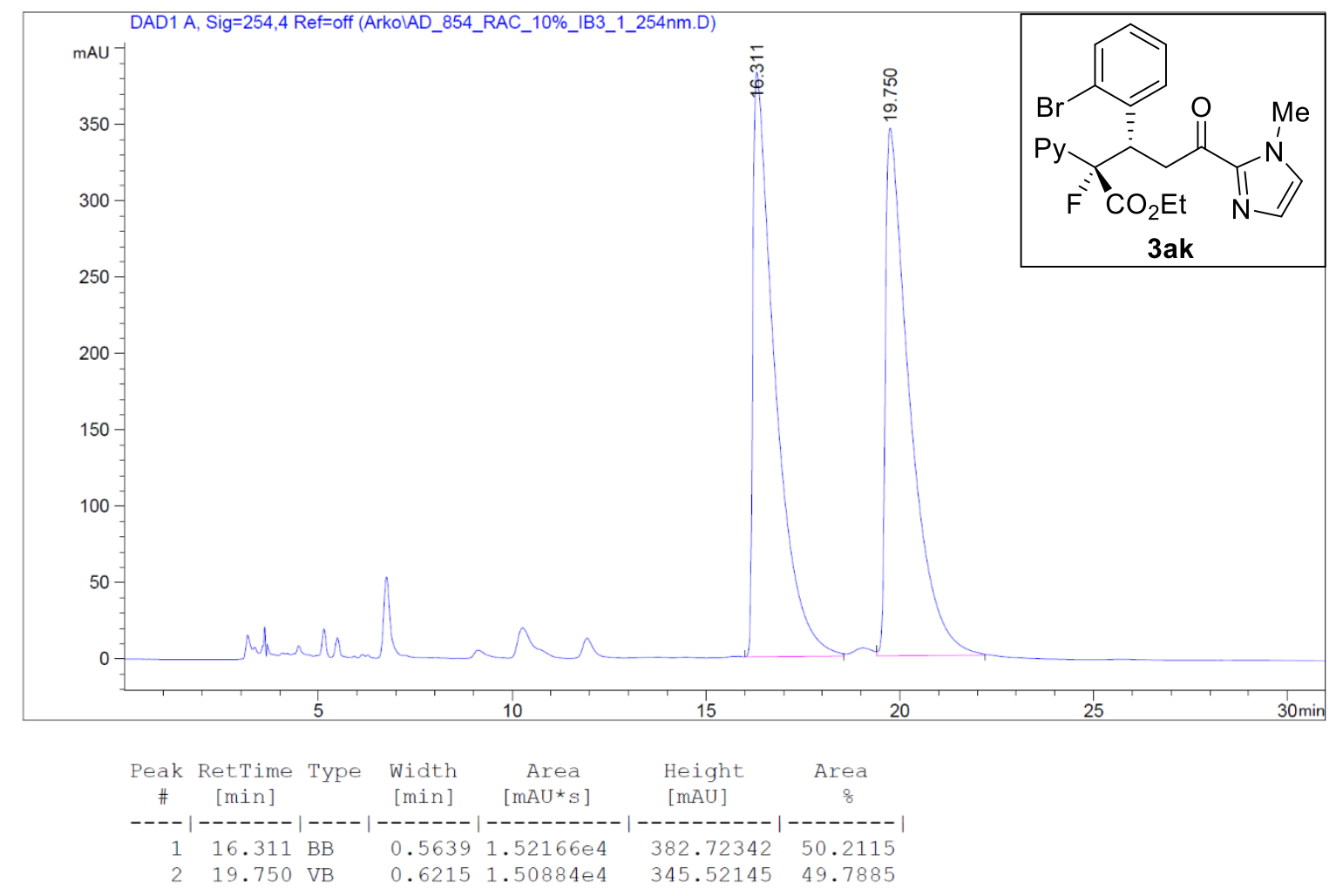

HPLC graph of racemic 3ak

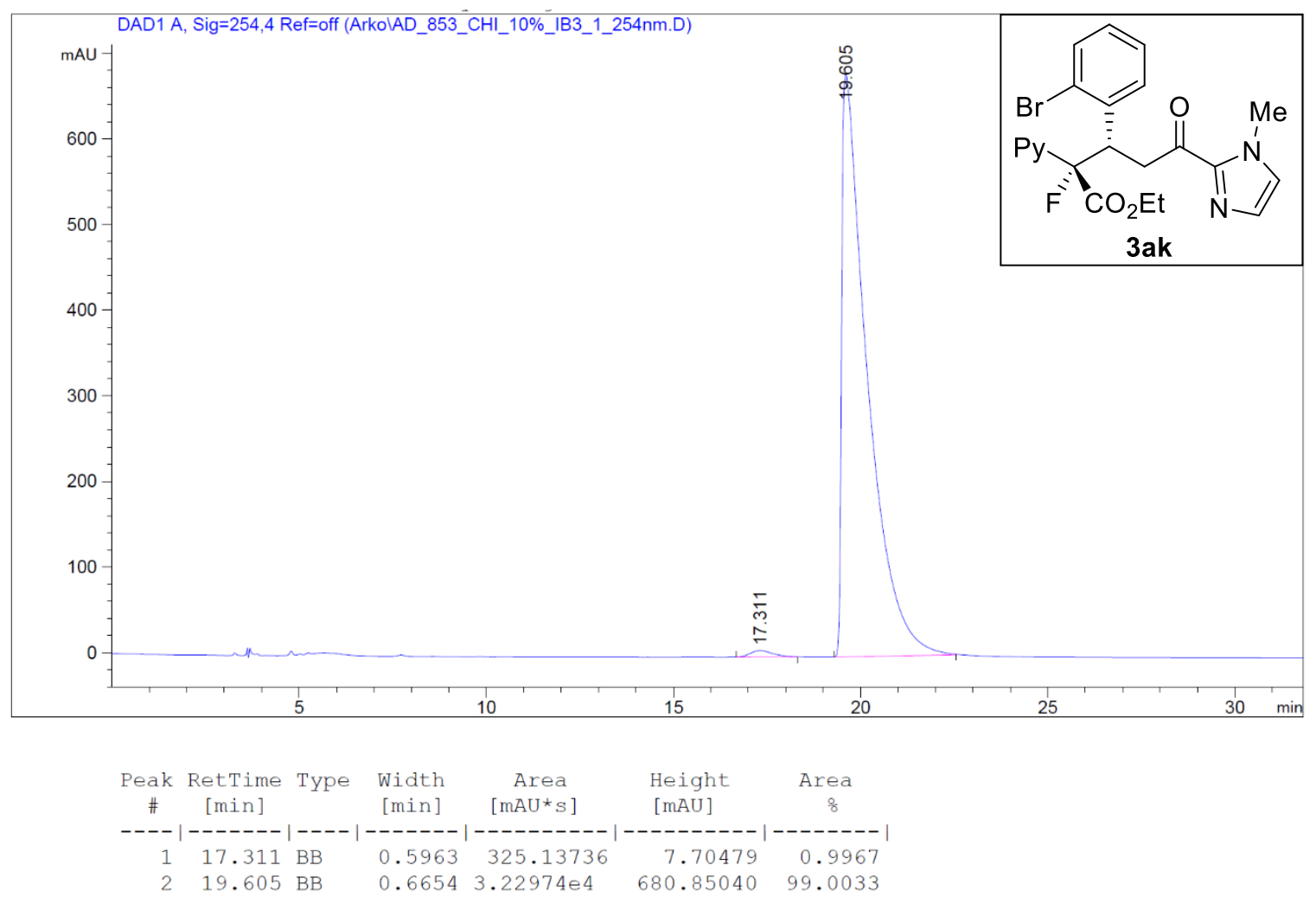

HPLC graph of chiral 3ak 


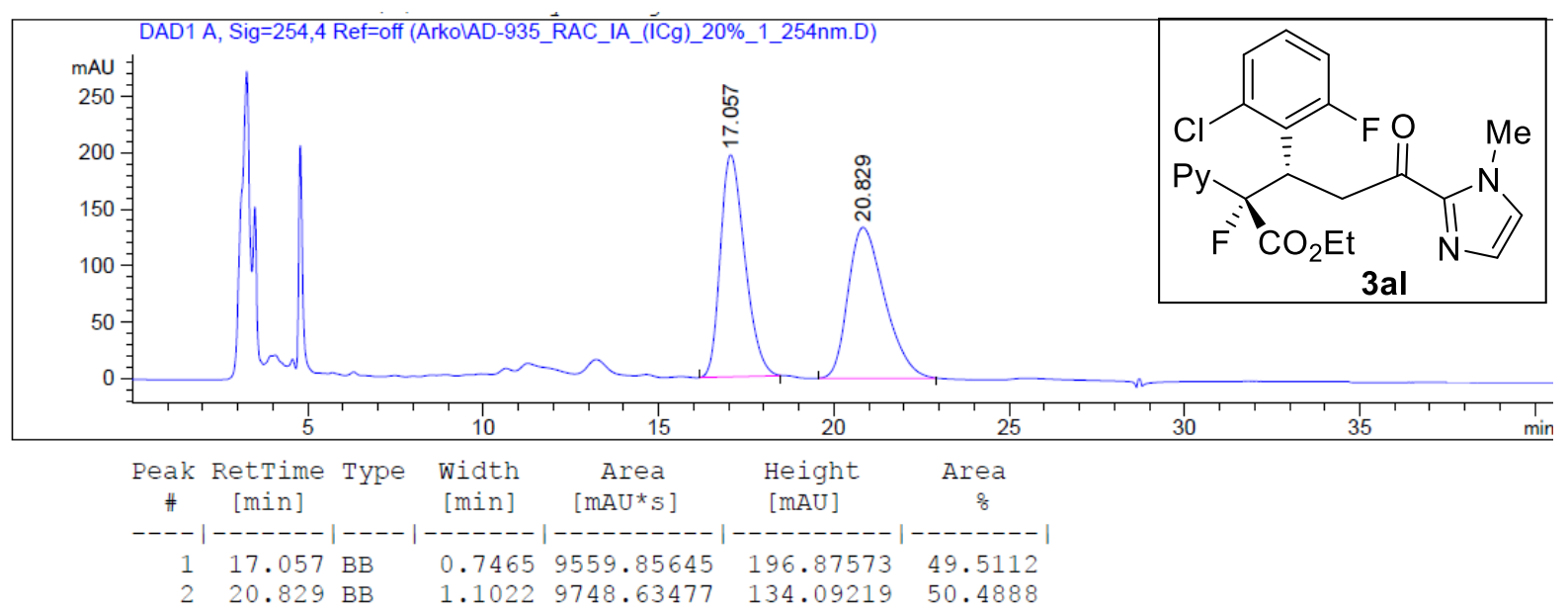

HPLC graph of racemic 3al

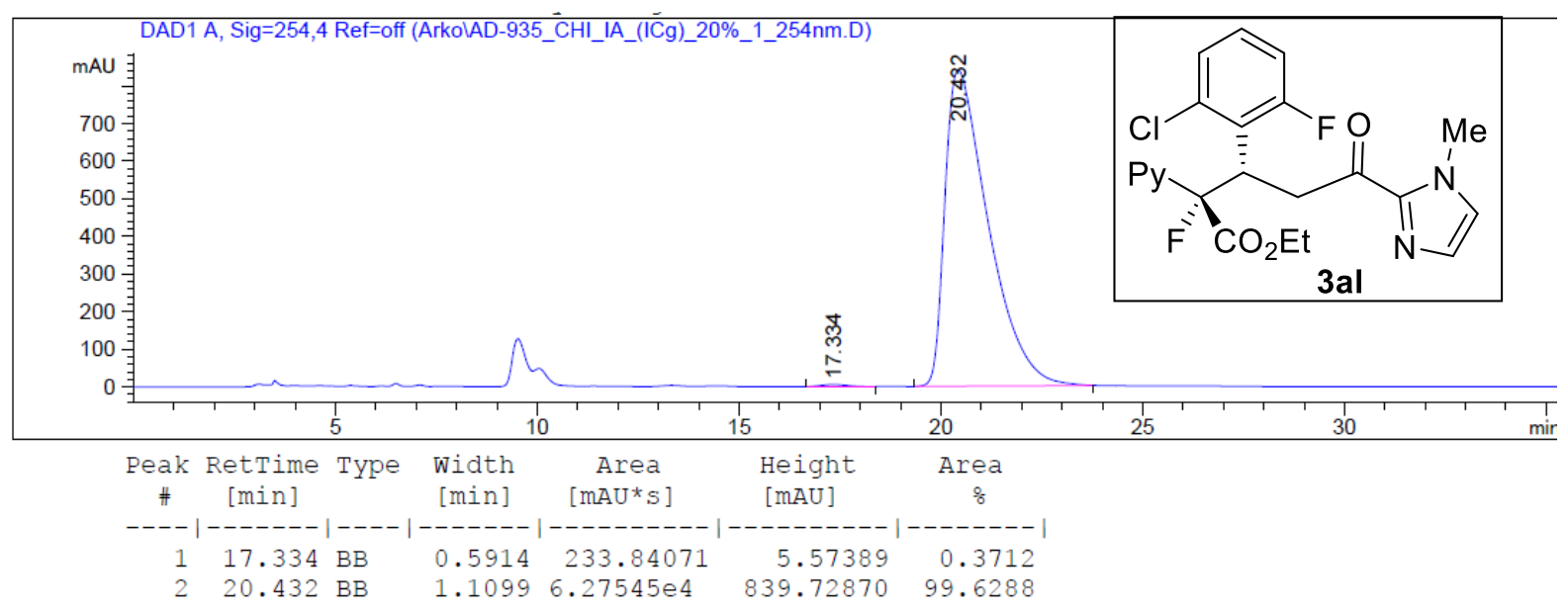

HPLC graph of chiral 3al 


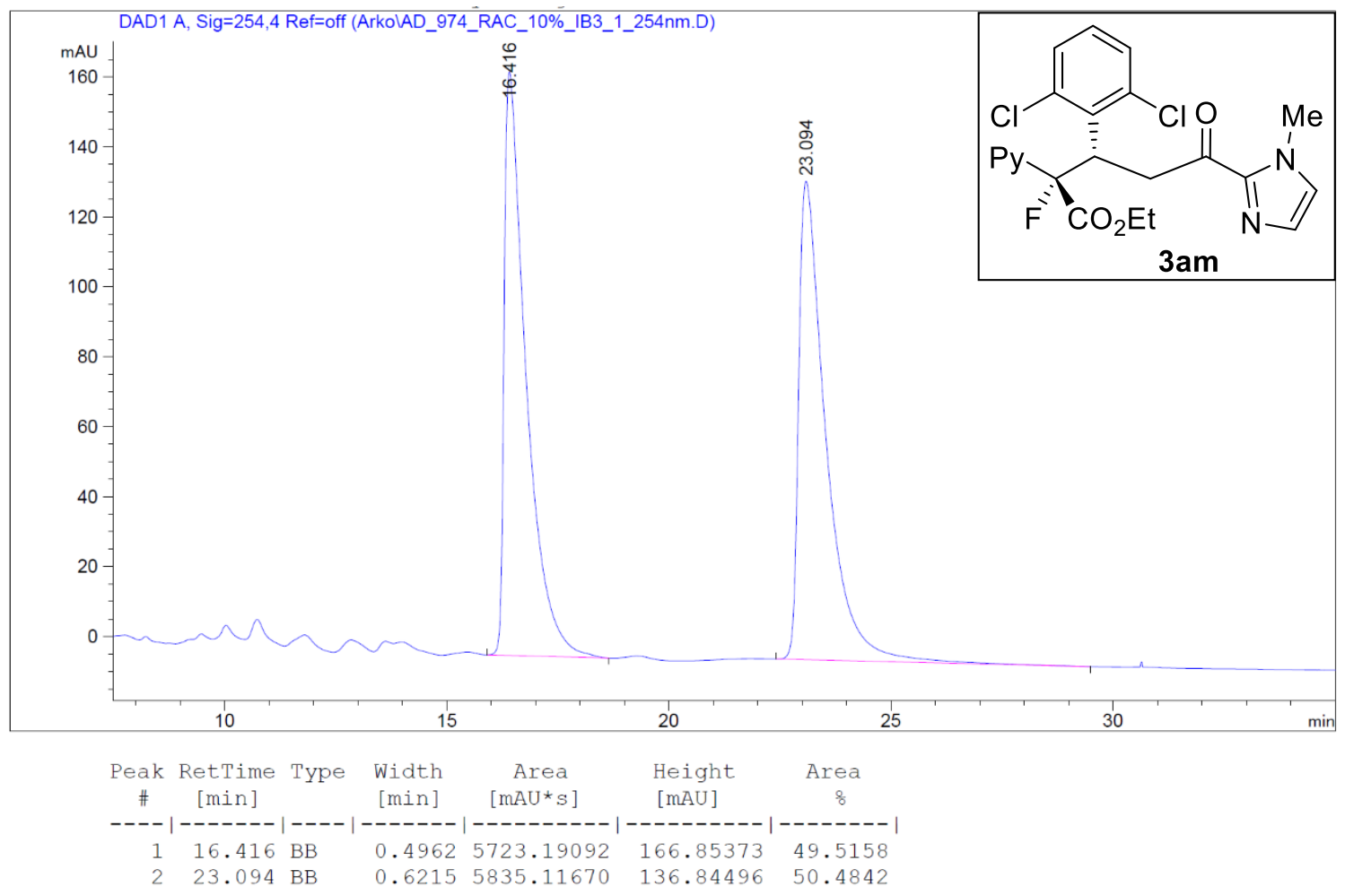

HPLC graph of racemic 3am

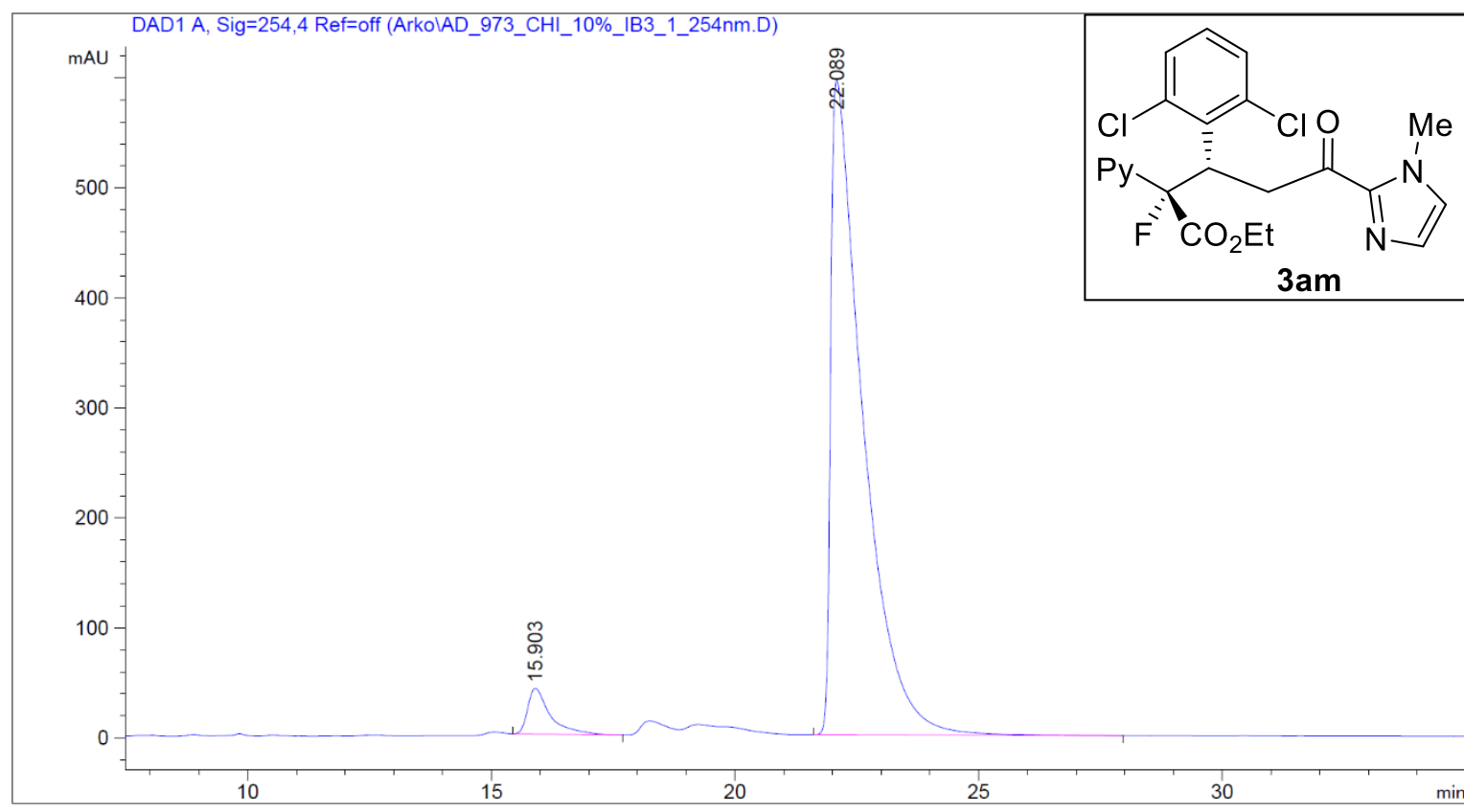

\begin{tabular}{|c|c|c|c|c|c|c|}
\hline $\begin{array}{c}\text { Peak } \\
\text { \# }\end{array}$ & $\begin{array}{c}\text { RetTime } \\
\text { [min] }\end{array}$ & Type & $\begin{array}{l}\text { Width } \\
{[\mathrm{min}]}\end{array}$ & $\begin{array}{c}\text { Area } \\
{\left[\mathrm{mAU}^{\star} \mathrm{S}\right]}\end{array}$ & $\begin{array}{l}\text { Height } \\
\text { [mAU] }\end{array}$ & $\begin{array}{c}\text { Area } \\
\frac{8}{8}\end{array}$ \\
\hline--1 & & & & --------- & --ー--- & ---- \\
\hline 1 & 15.903 & $\mathrm{BB}$ & 0.4628 & 1320.80884 & 41.55793 & 4.6088 \\
\hline 2 & 22.089 & $\mathrm{BB}$ & 0.6580 & $2.73379 e 4$ & 595.02301 & 95.3912 \\
\hline
\end{tabular}

HPLC graph of chiral 3am 


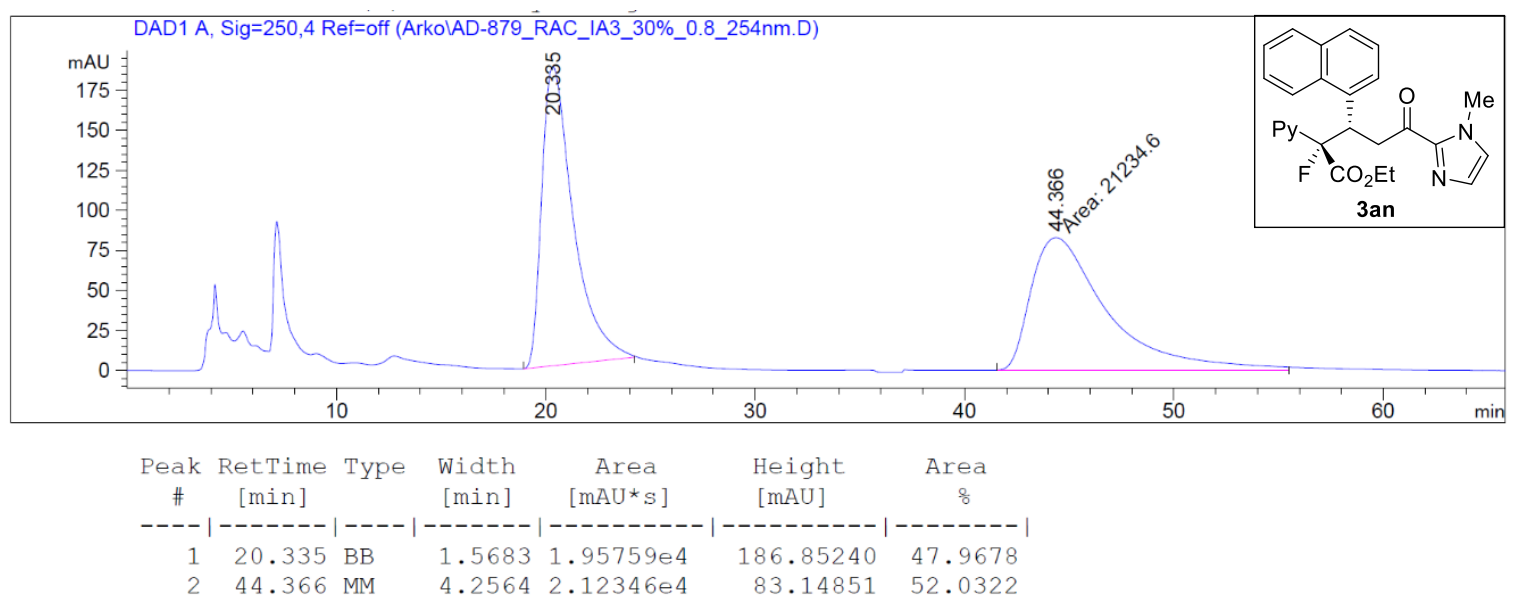

HPLC graph of racemic 3an

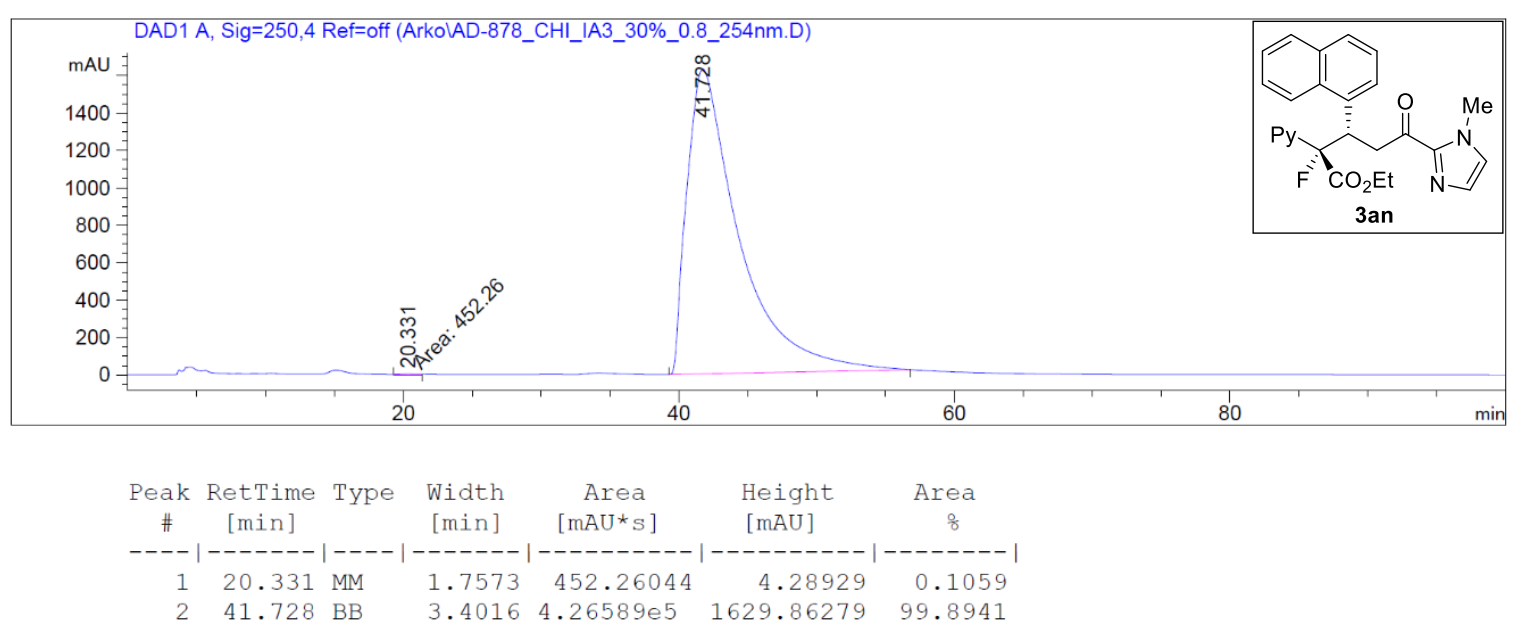

HPLC graph of chiral 3an 


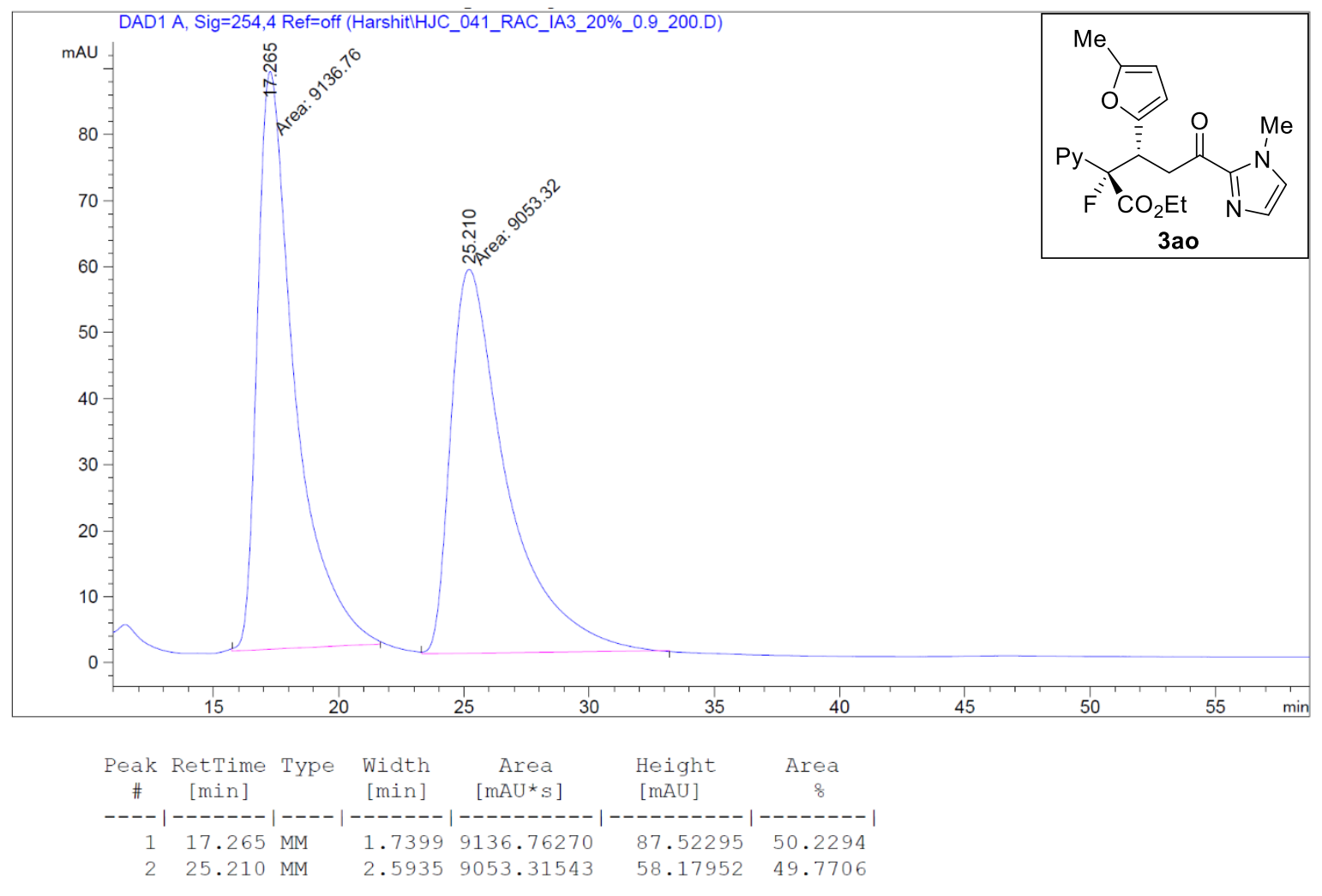

HPLC graph of racemic 3ao

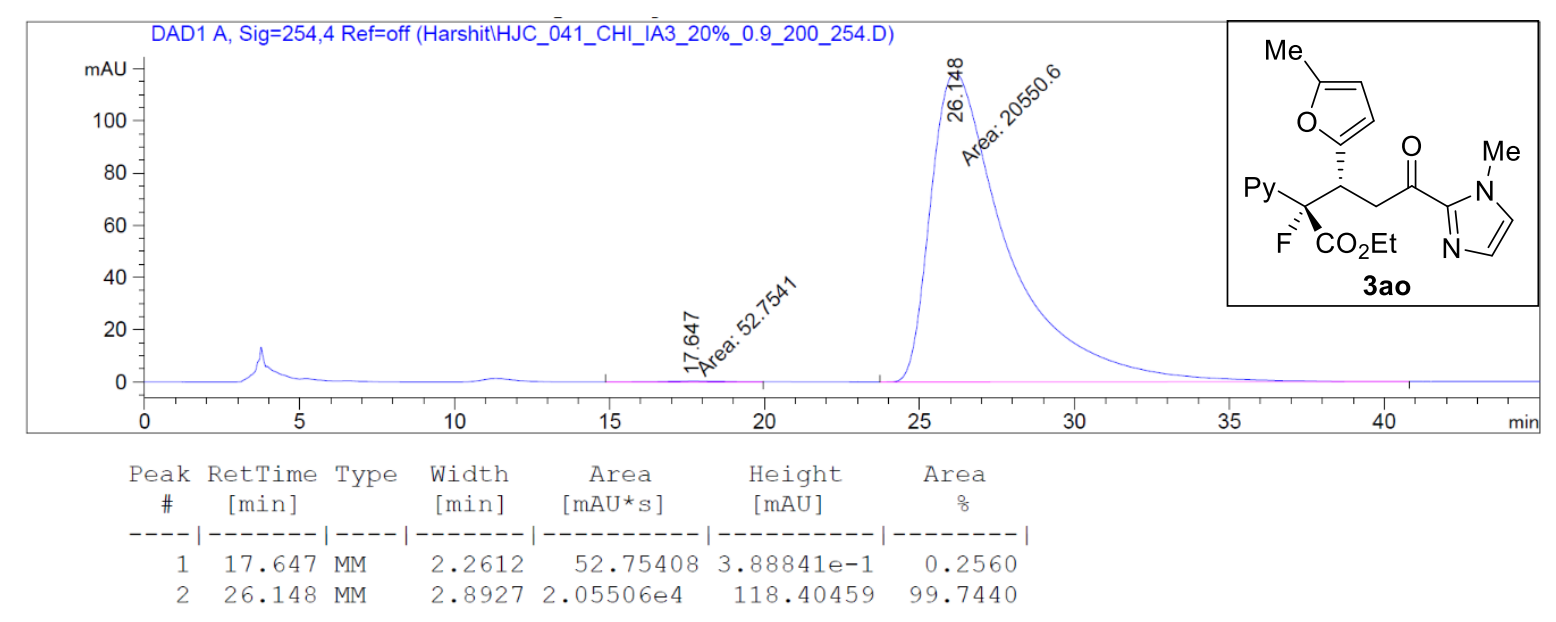

HPLC graph of chiral 3ao 


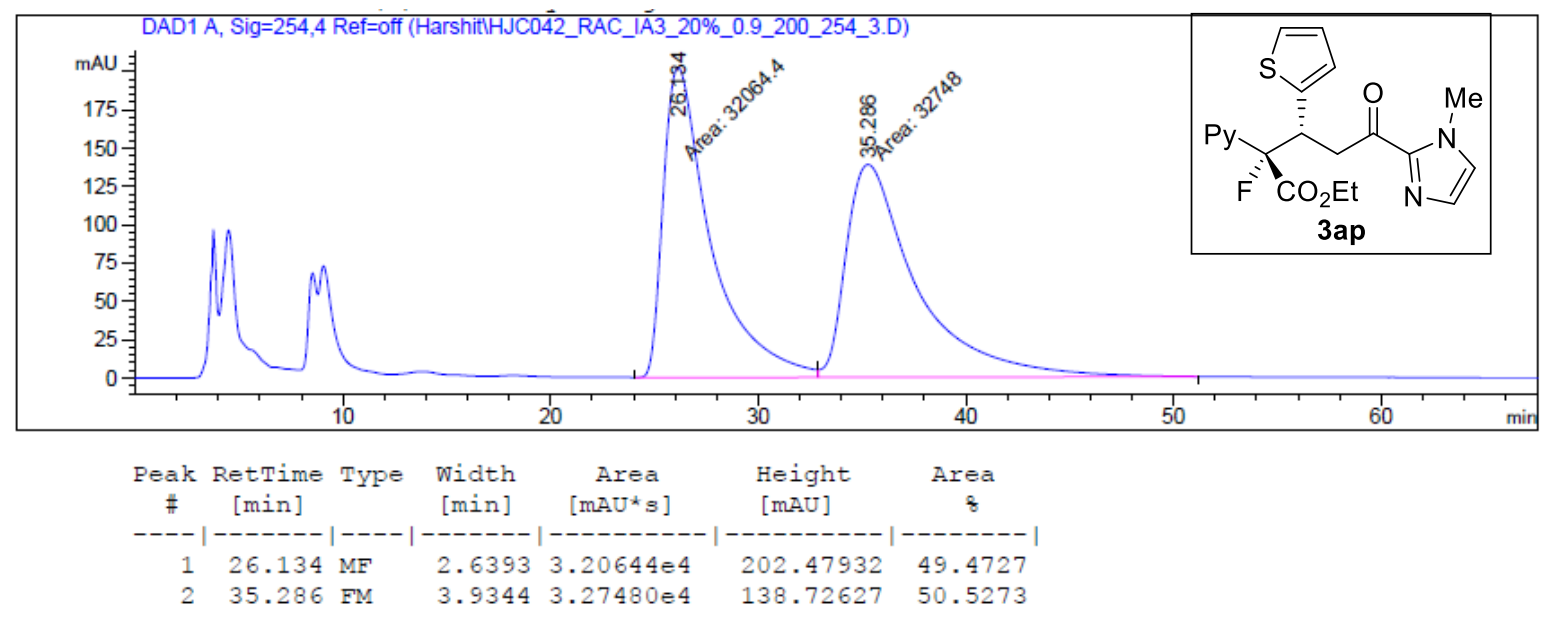

HPLC graph of racemic 3ap

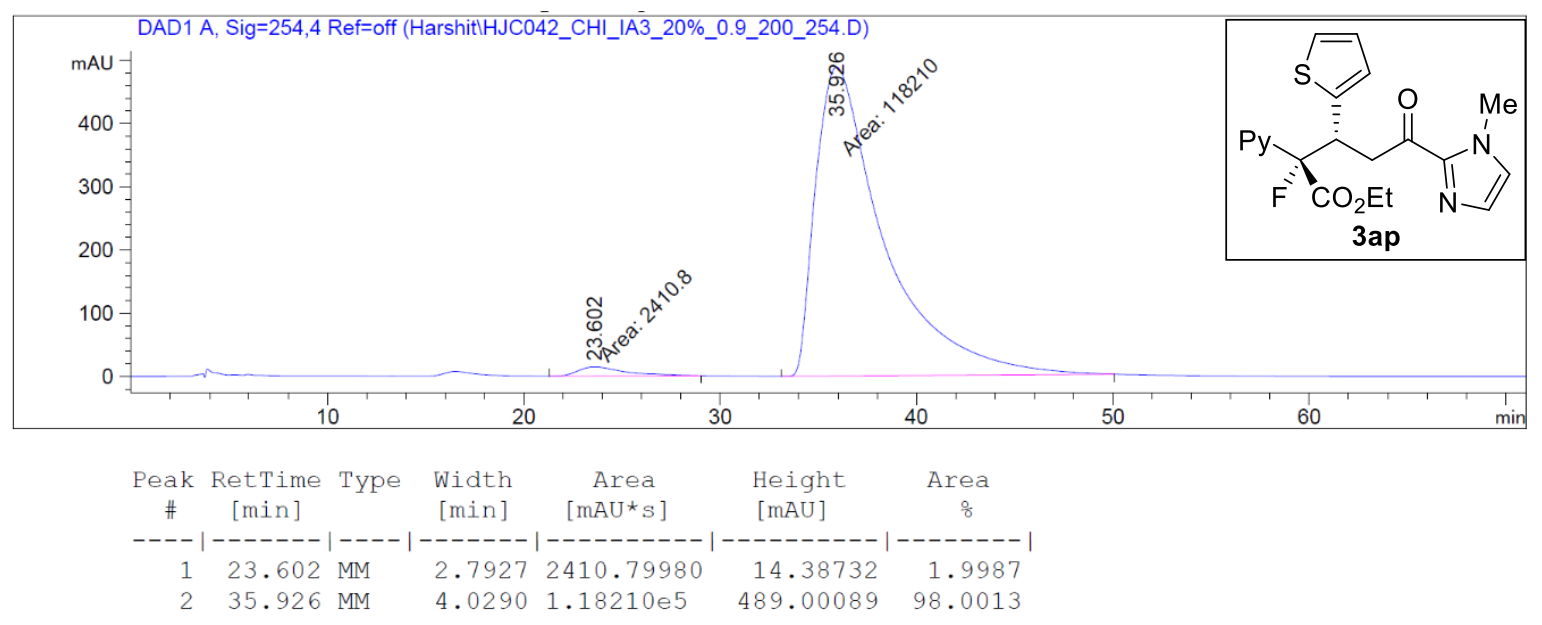

HPLC graph of chiral 3ap 


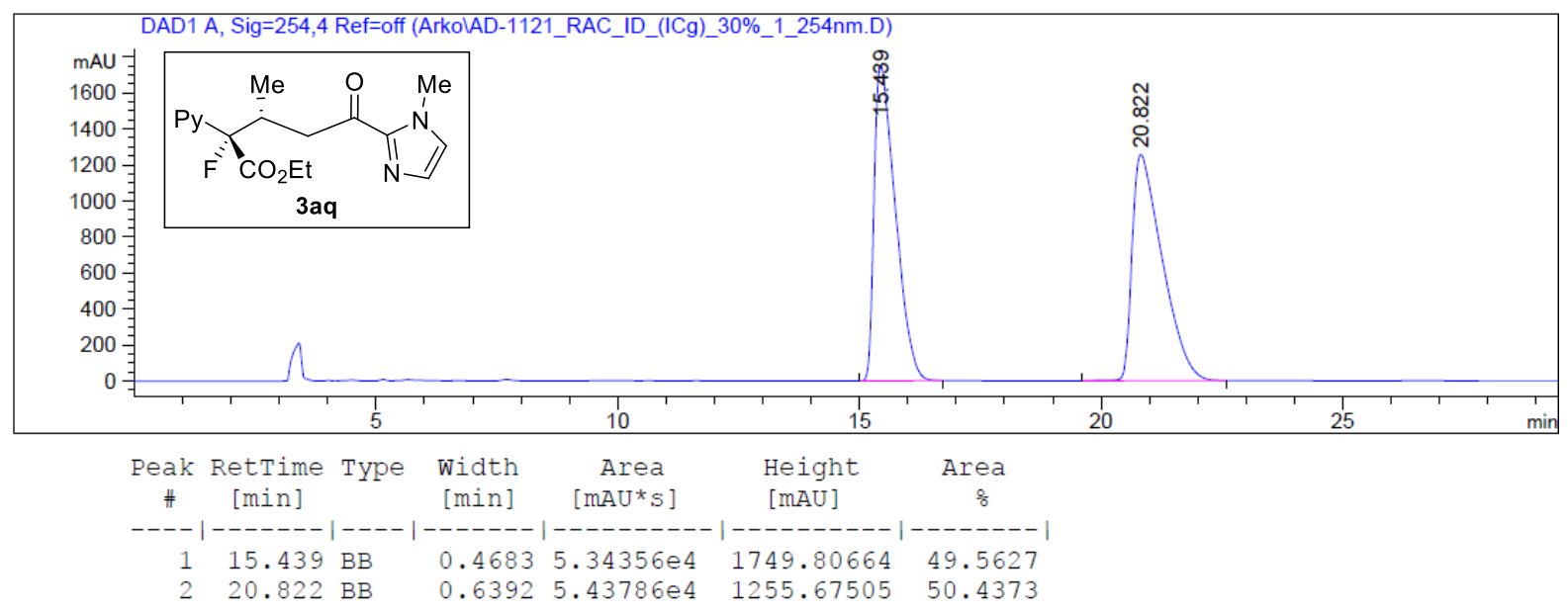

HPLC graph of racemic 3aq

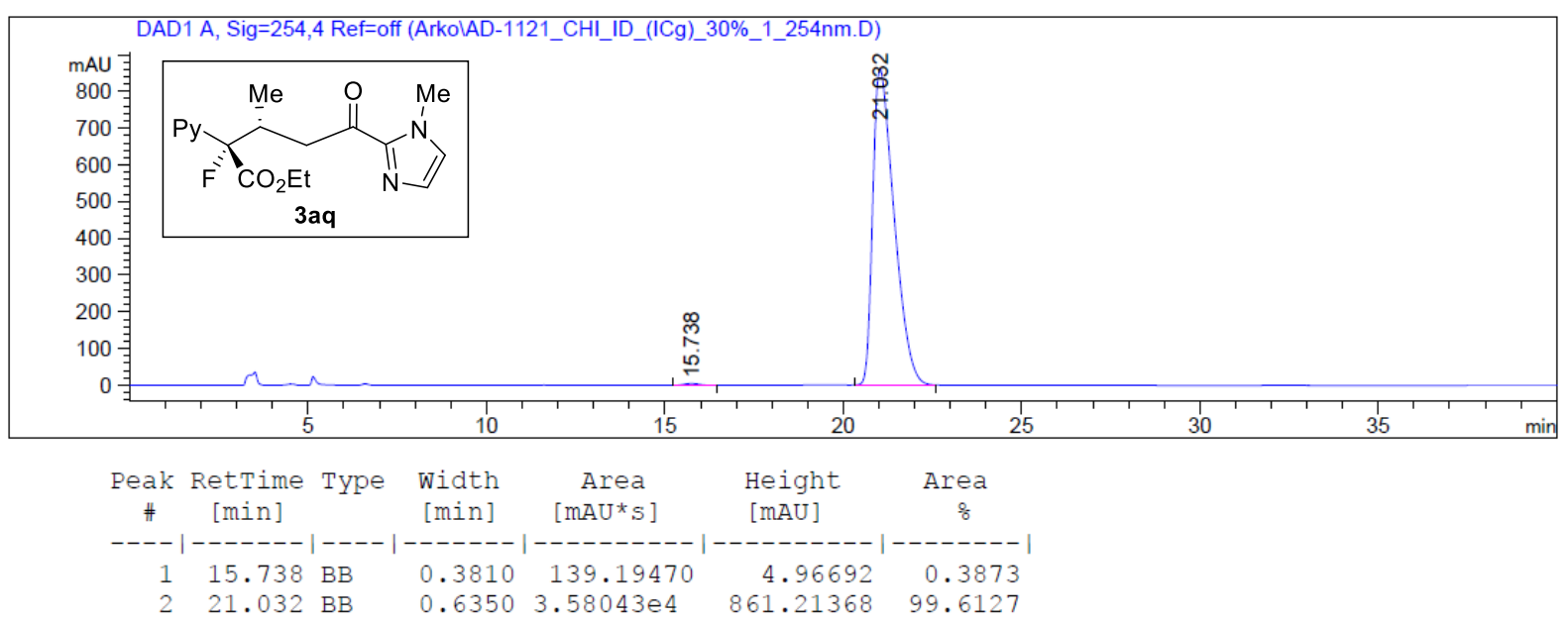

HPLC graph of chiral 3aq 


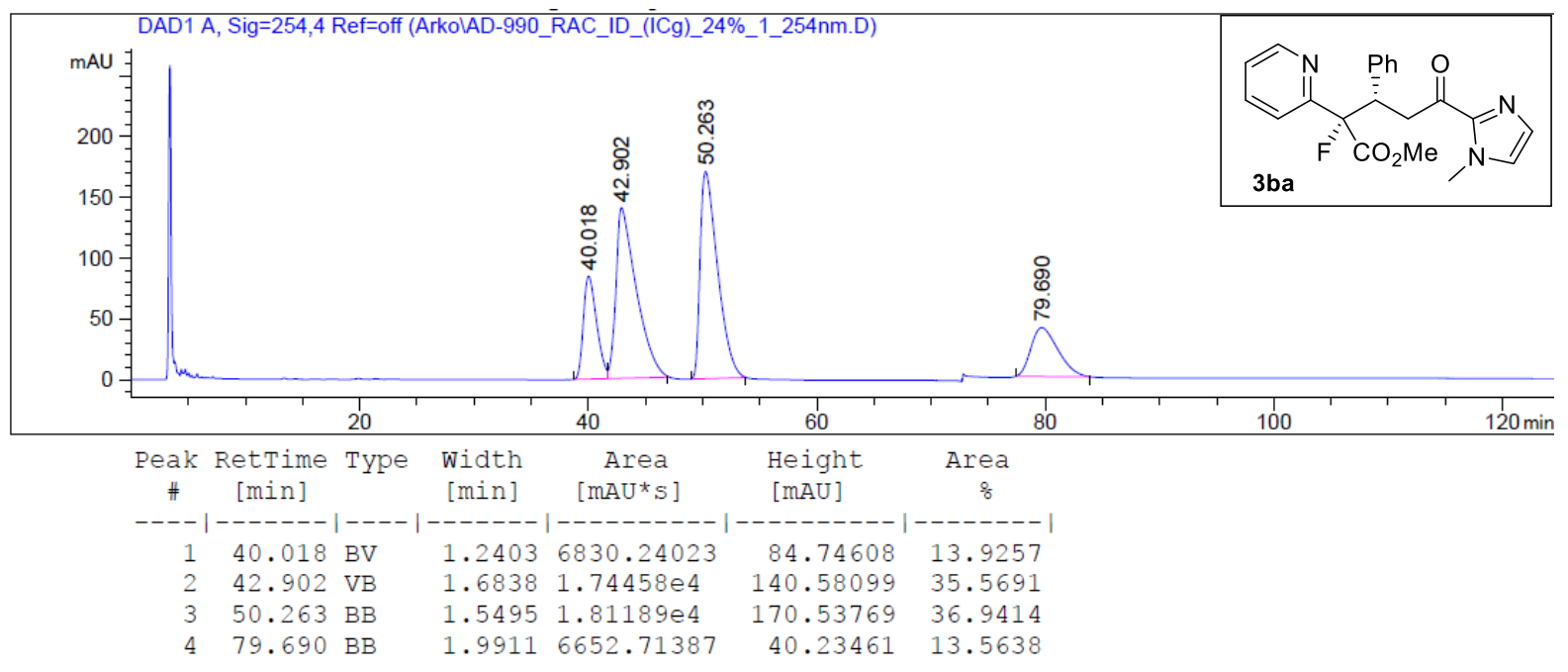

HPLC graph of racemic 3 ba

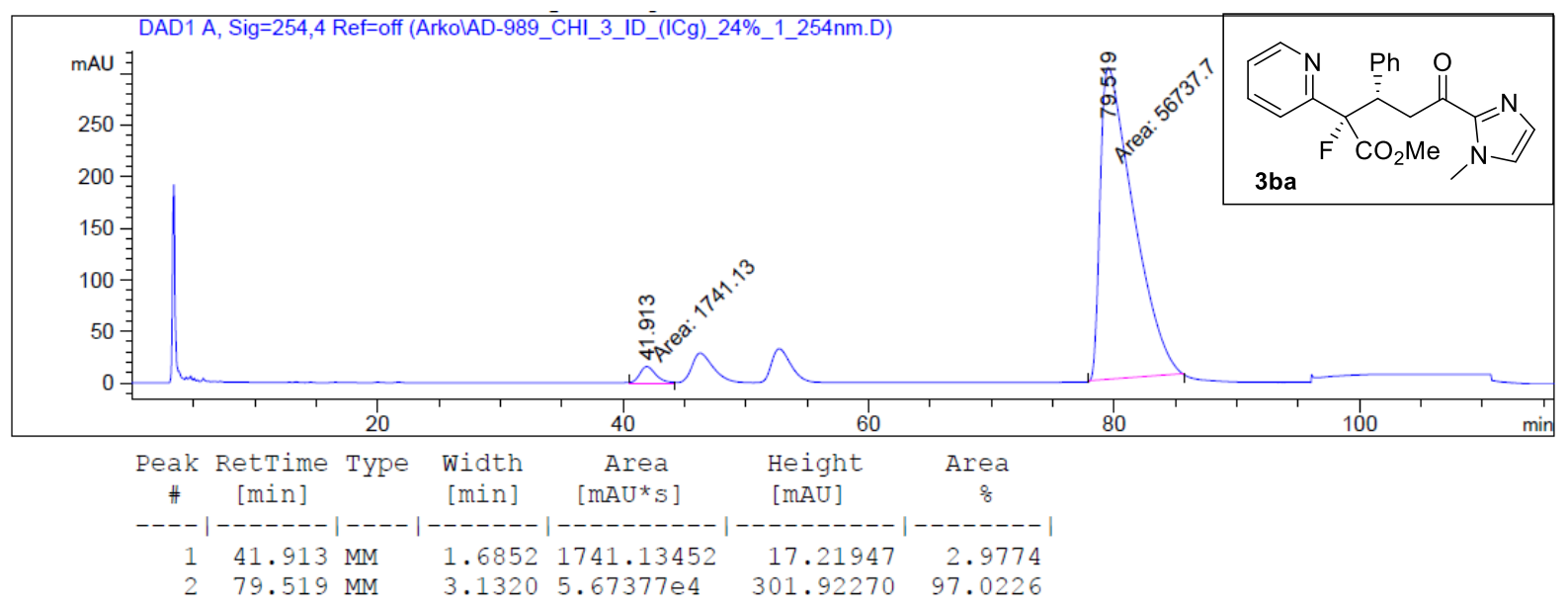

HPLC graph of chiral 3ba 


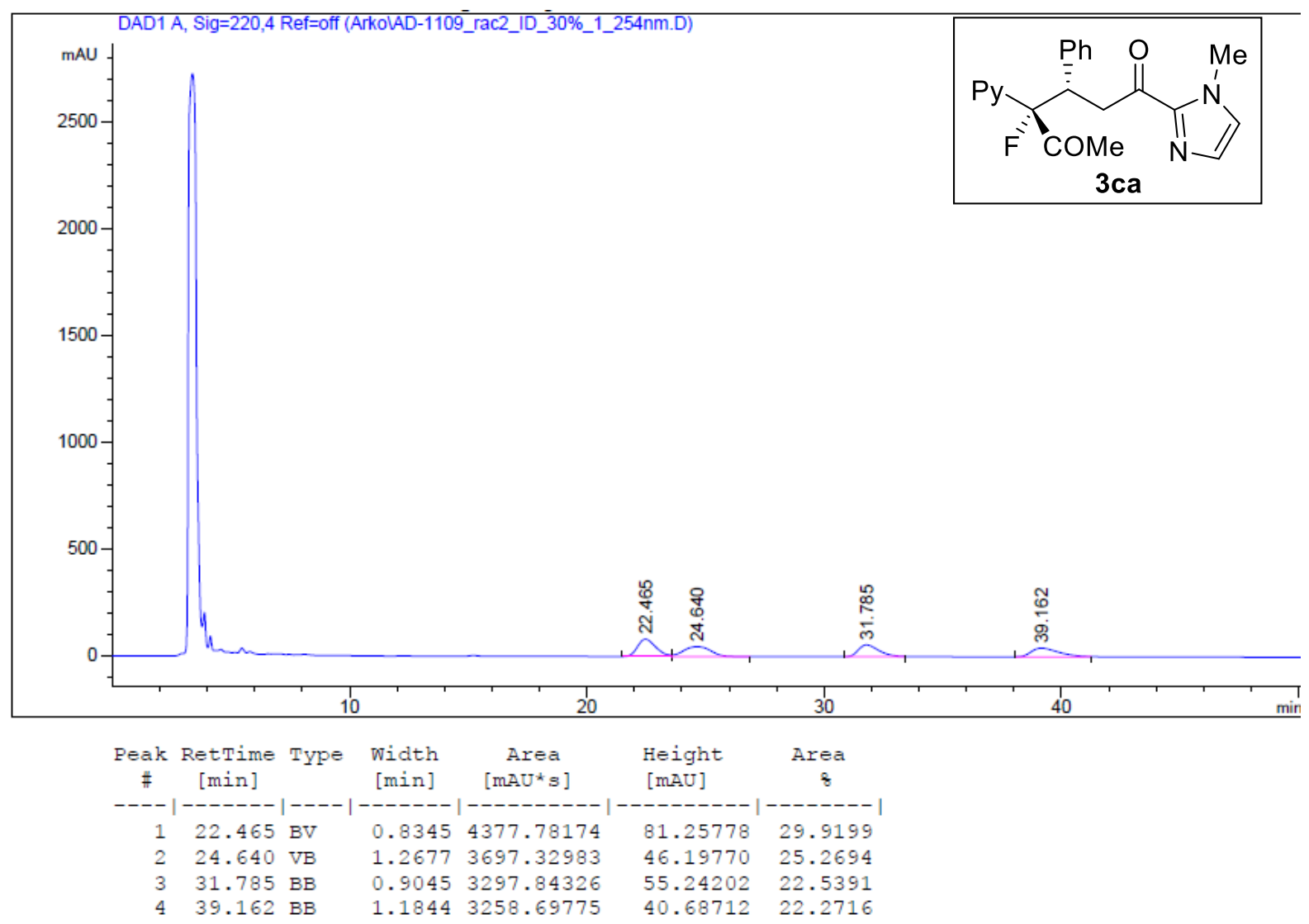

HPLC graph of racemic $3 \mathbf{c a}$

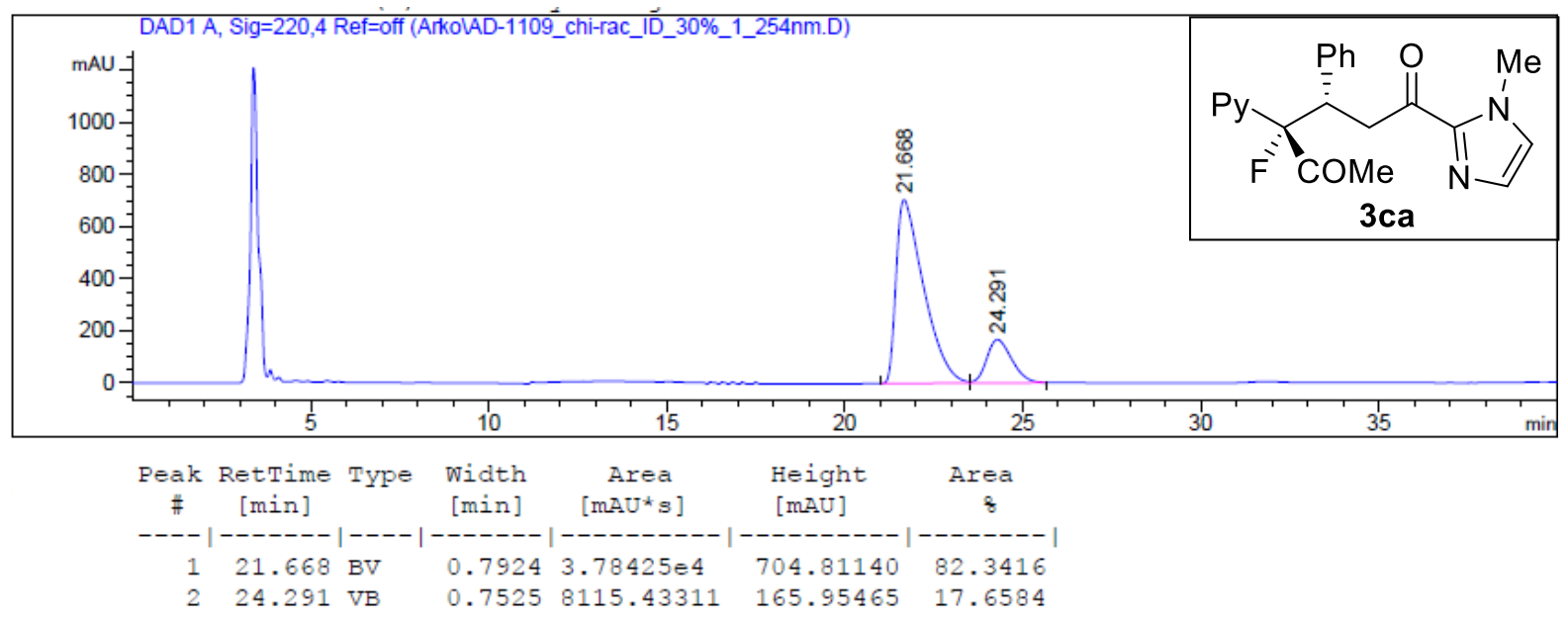

HPLC graph of chiral 3ca 


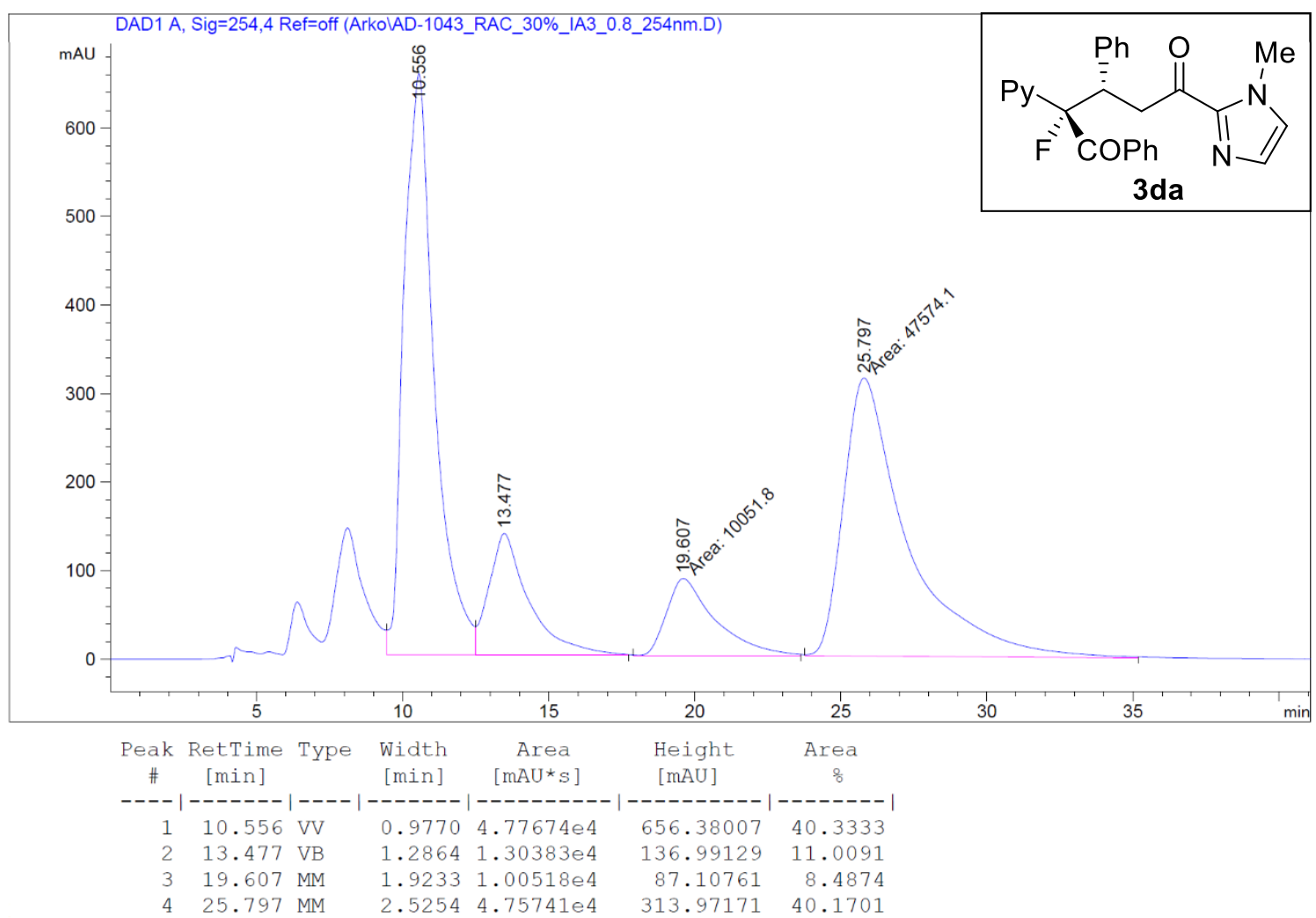

HPLC graph of racemic 3 da

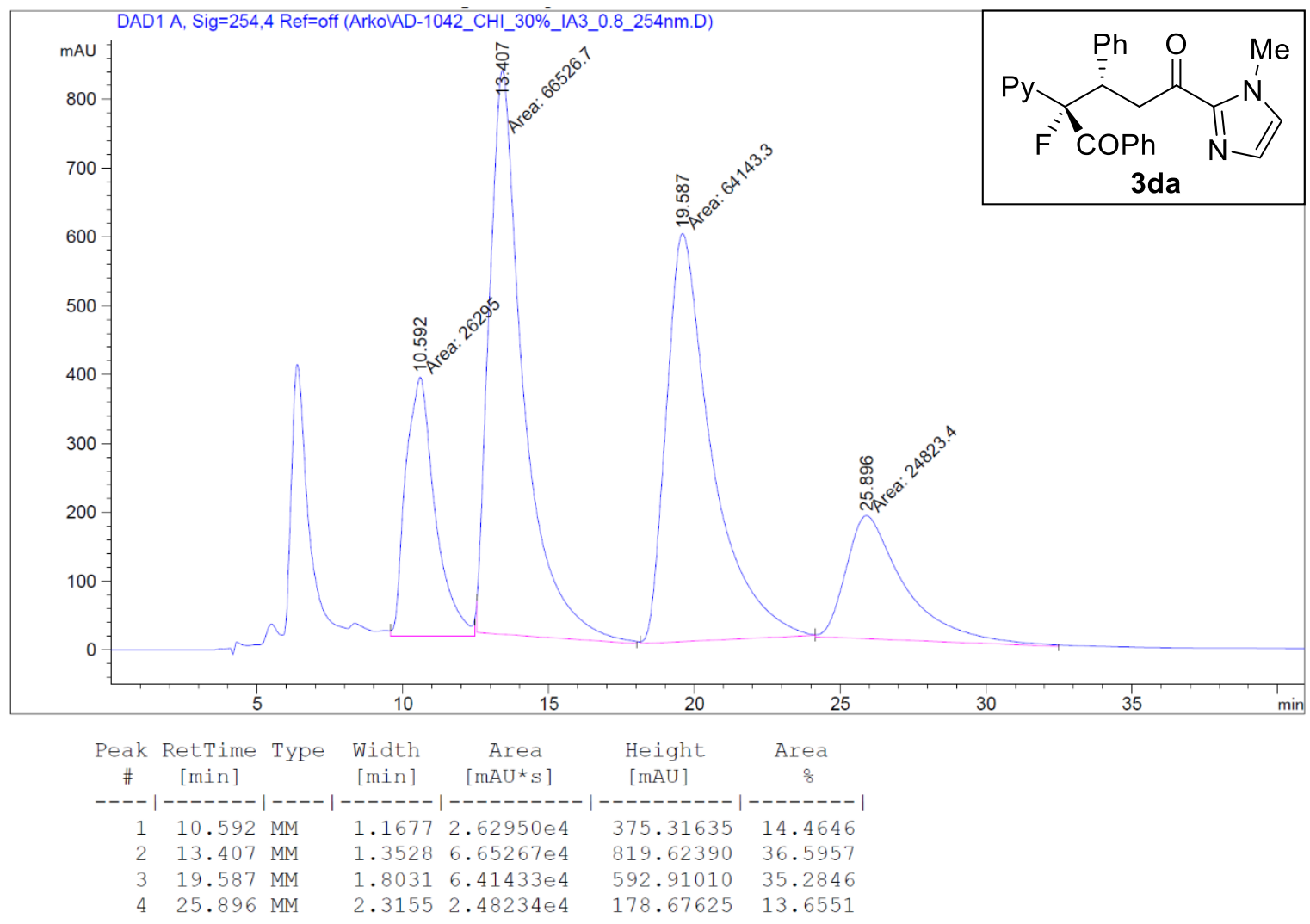

HPLC graph of chiral 3da 


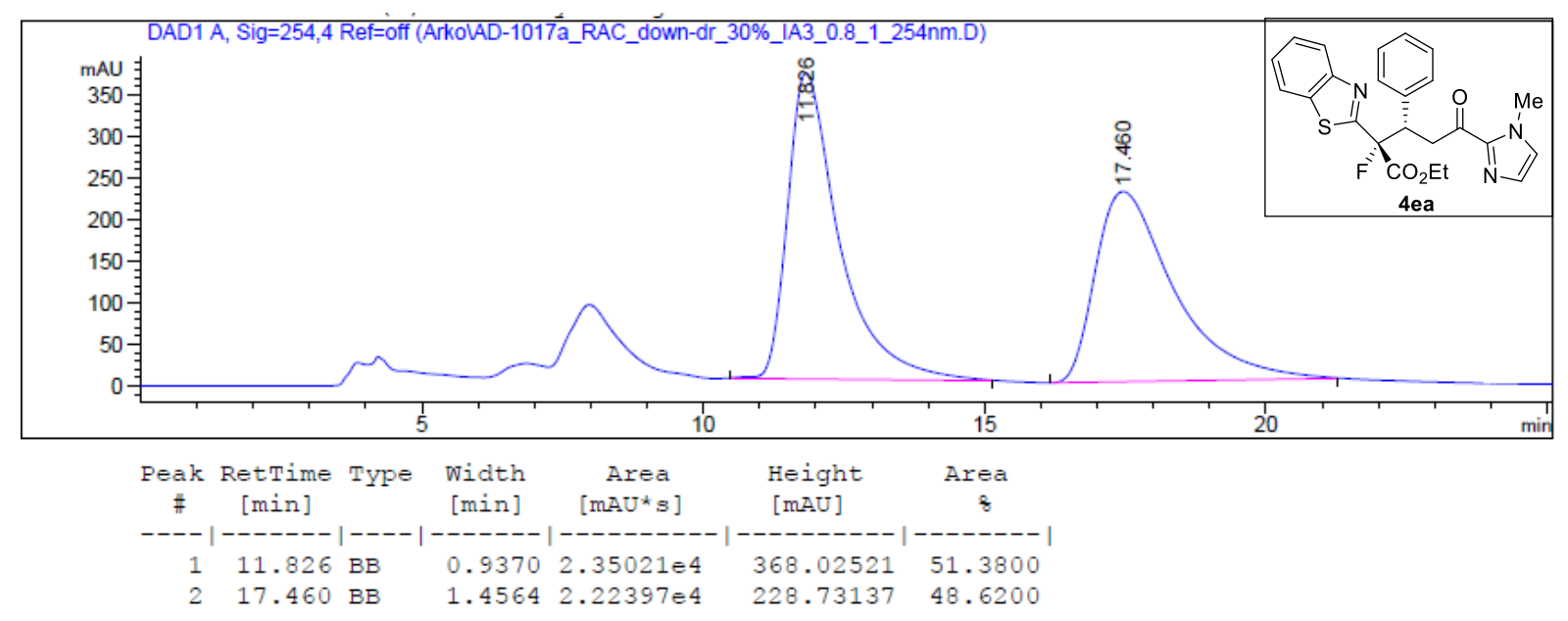

HPLC graph of racemic 4ea

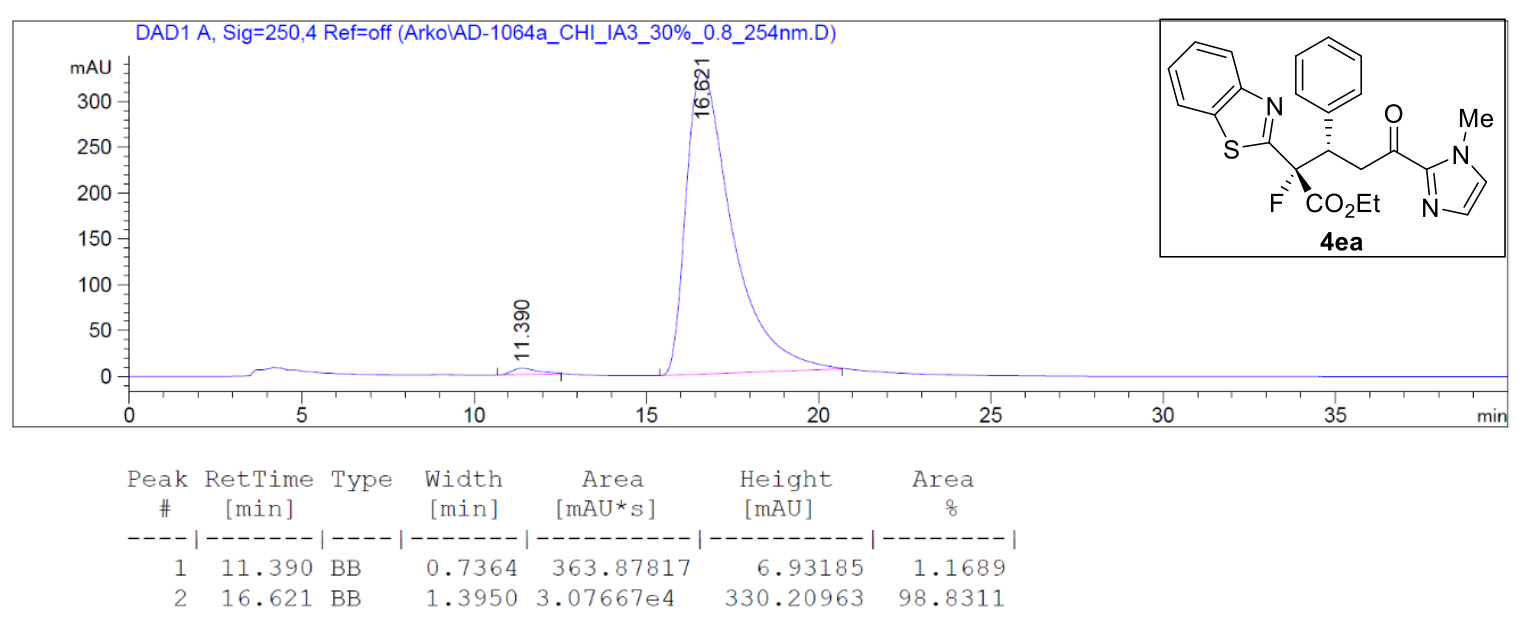

HPLC graph of chiral 4ea 


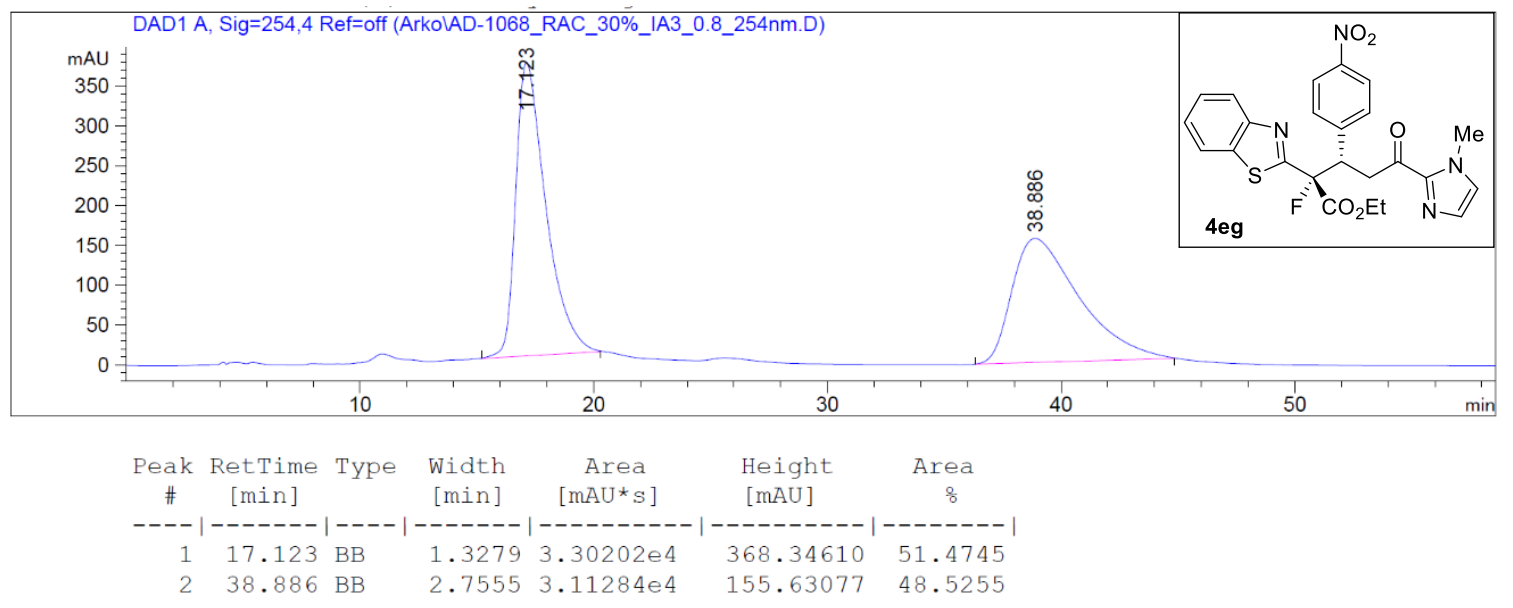

HPLC graph of racemic $4 \mathrm{eg}$

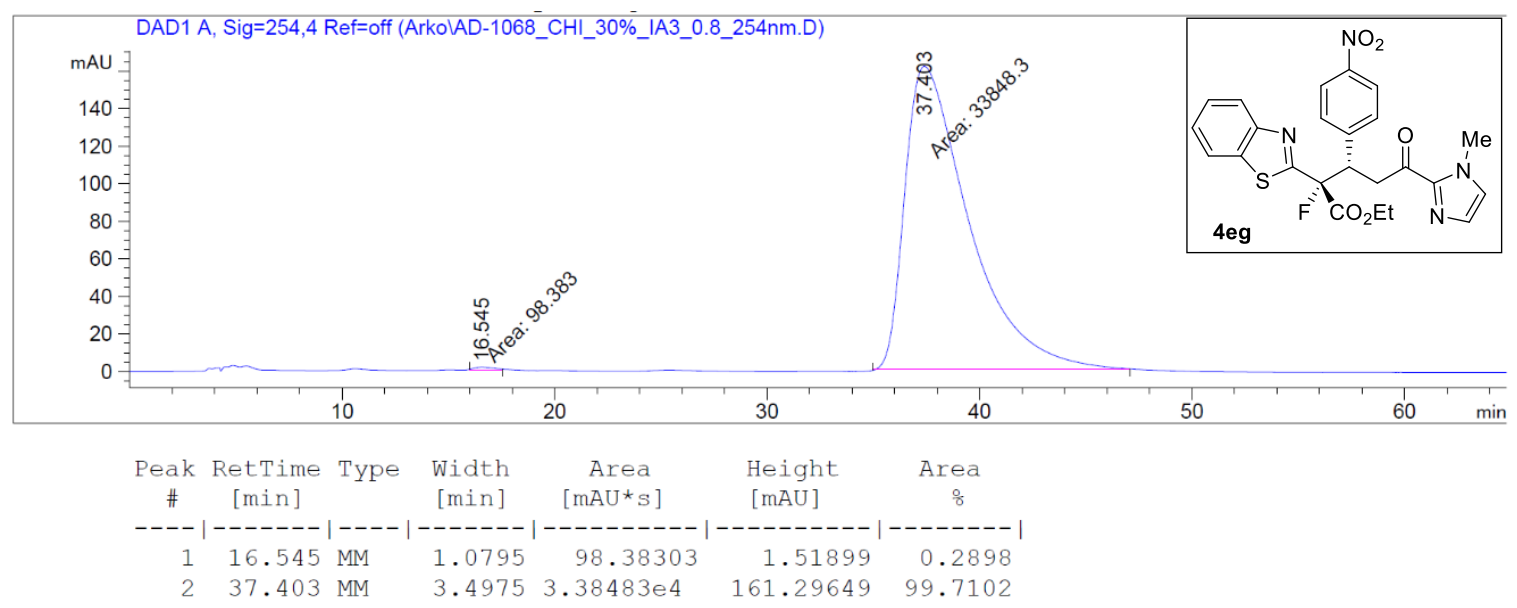

HPLC graph of chiral $\mathbf{4 e g}$ 


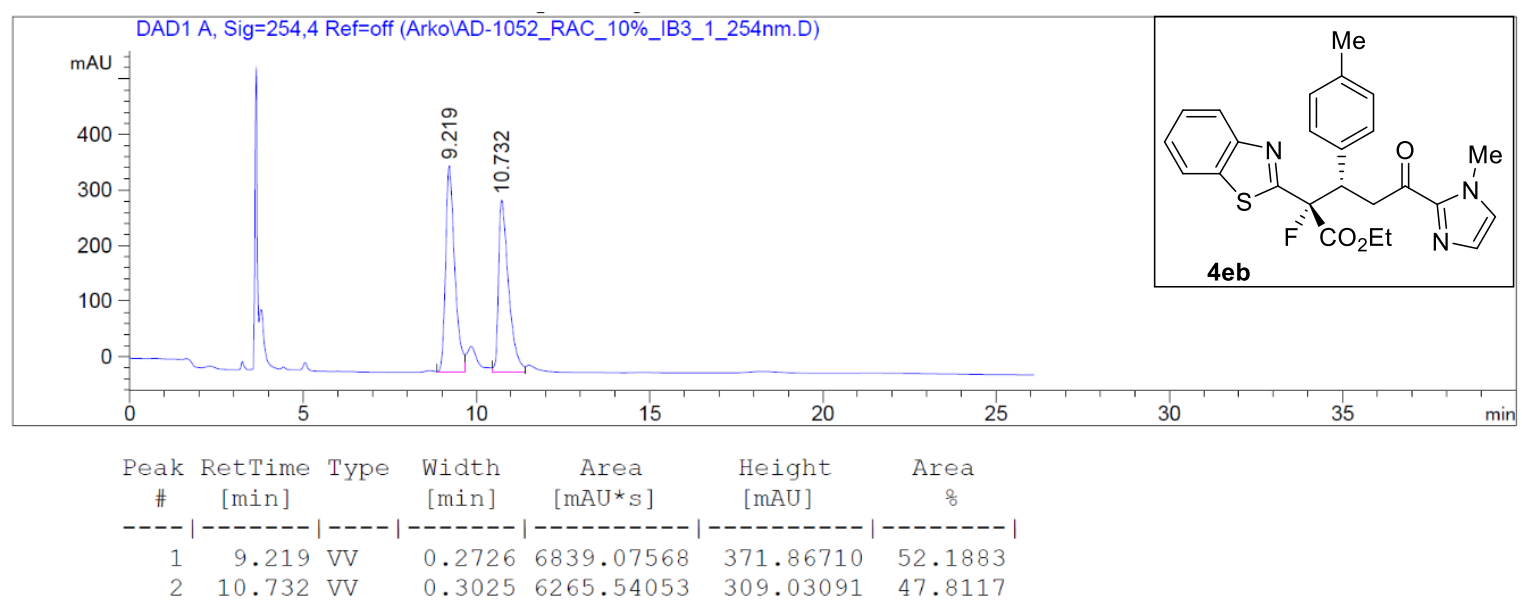

HPLC graph of racemic $4 \mathrm{eb}$

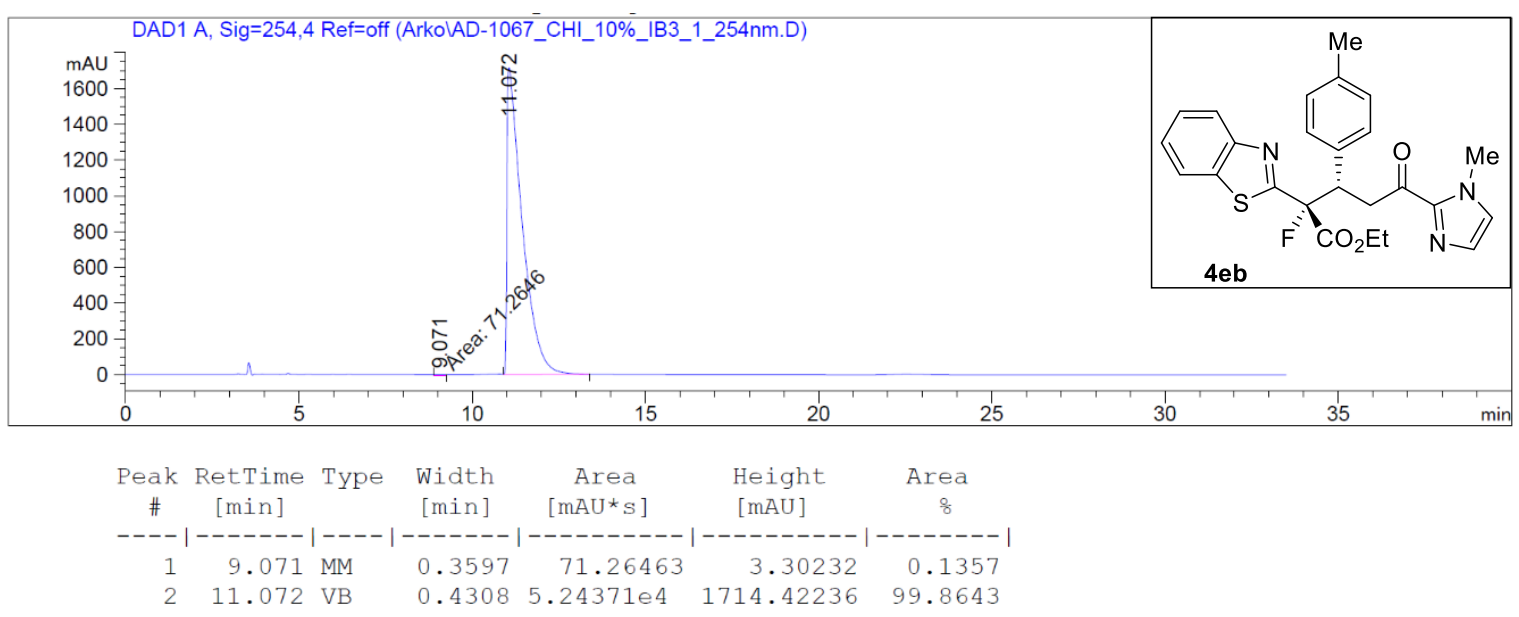

HPLC graph of chiral $4 \mathrm{eb}$ 


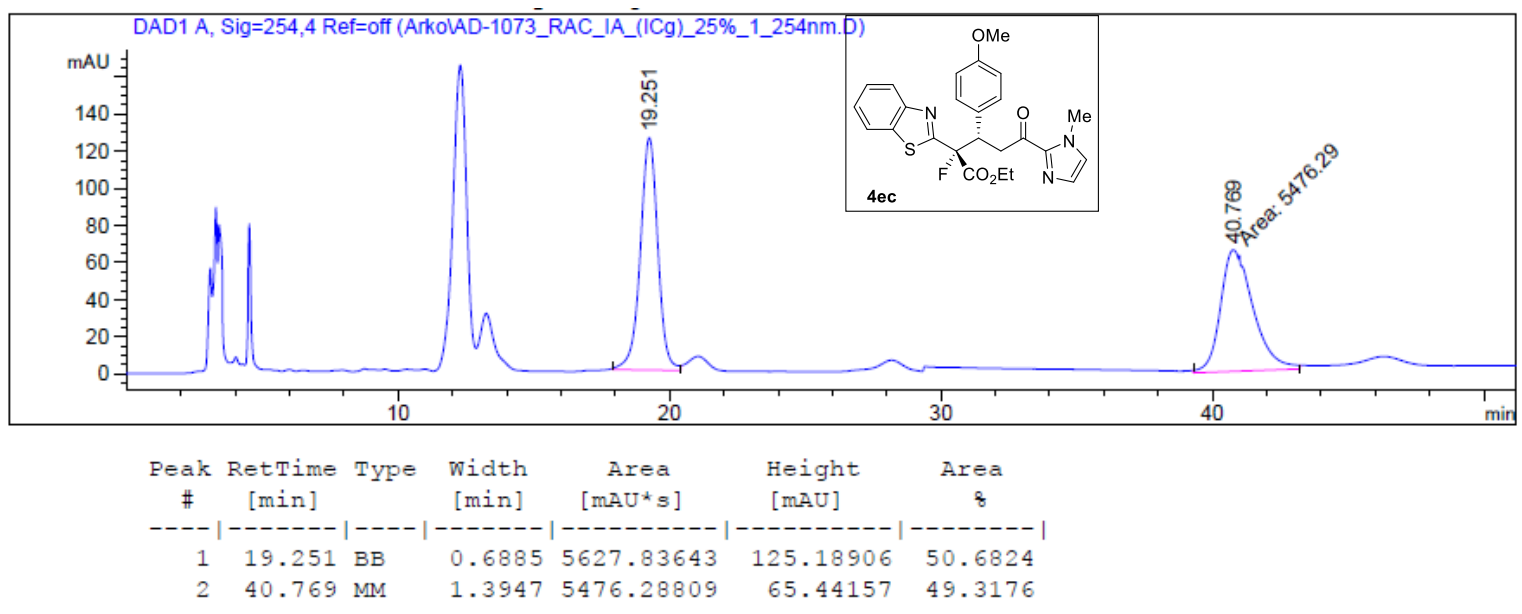

HPLC graph of racemic $4 \mathrm{ec}$

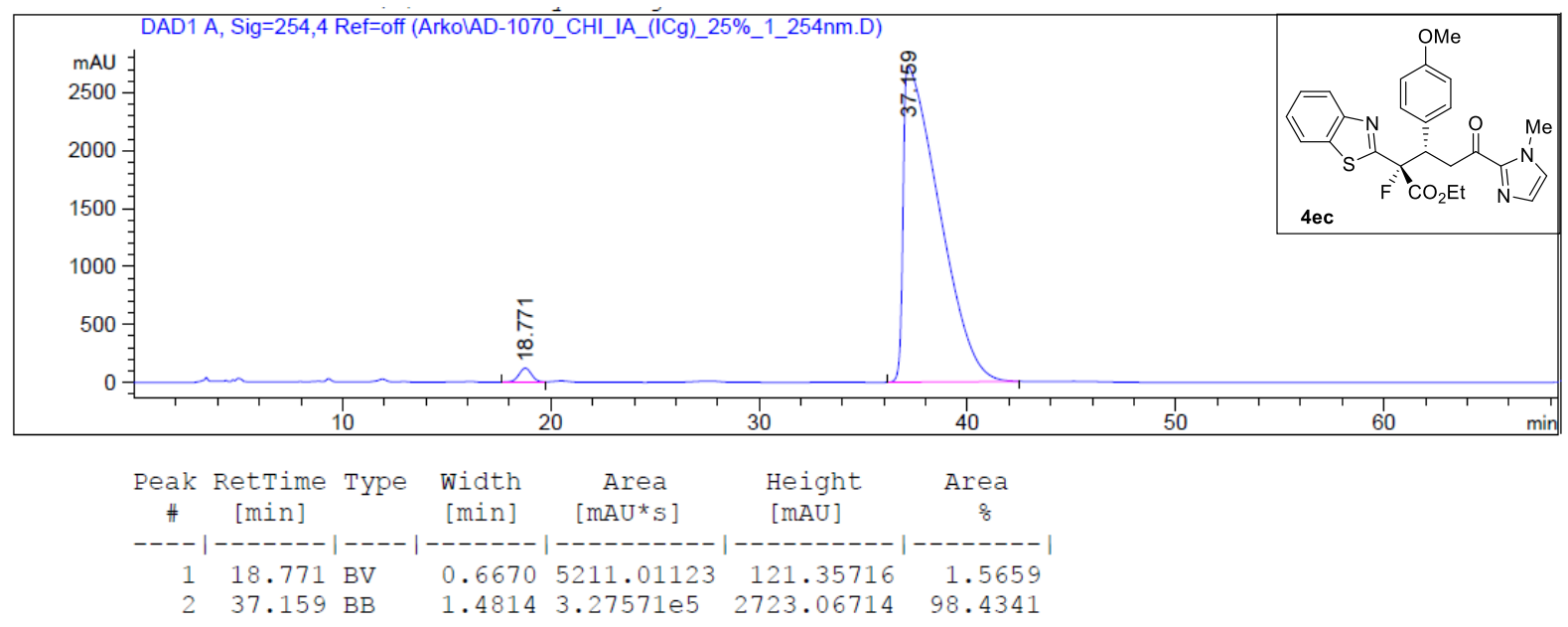

HPLC graph of chiral $4 \mathrm{ec}$ 

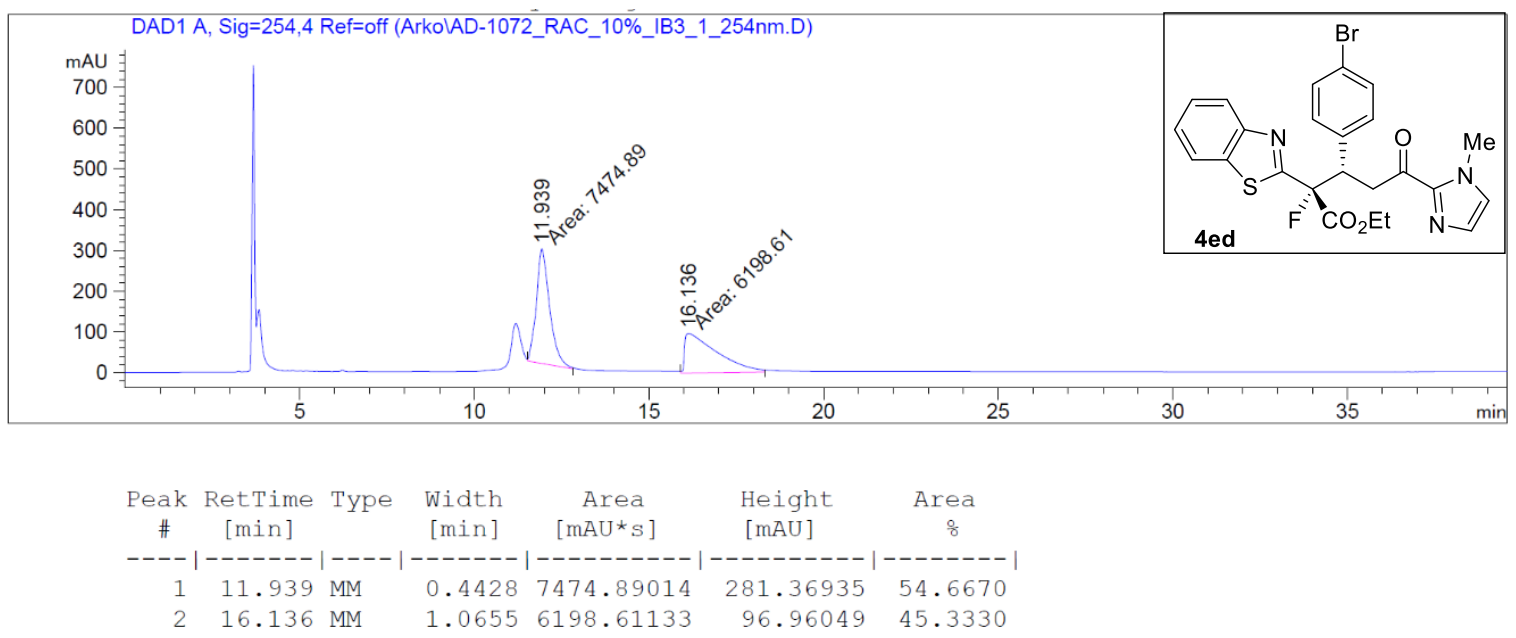

HPLC graph of racemic $4 \mathbf{e d}$

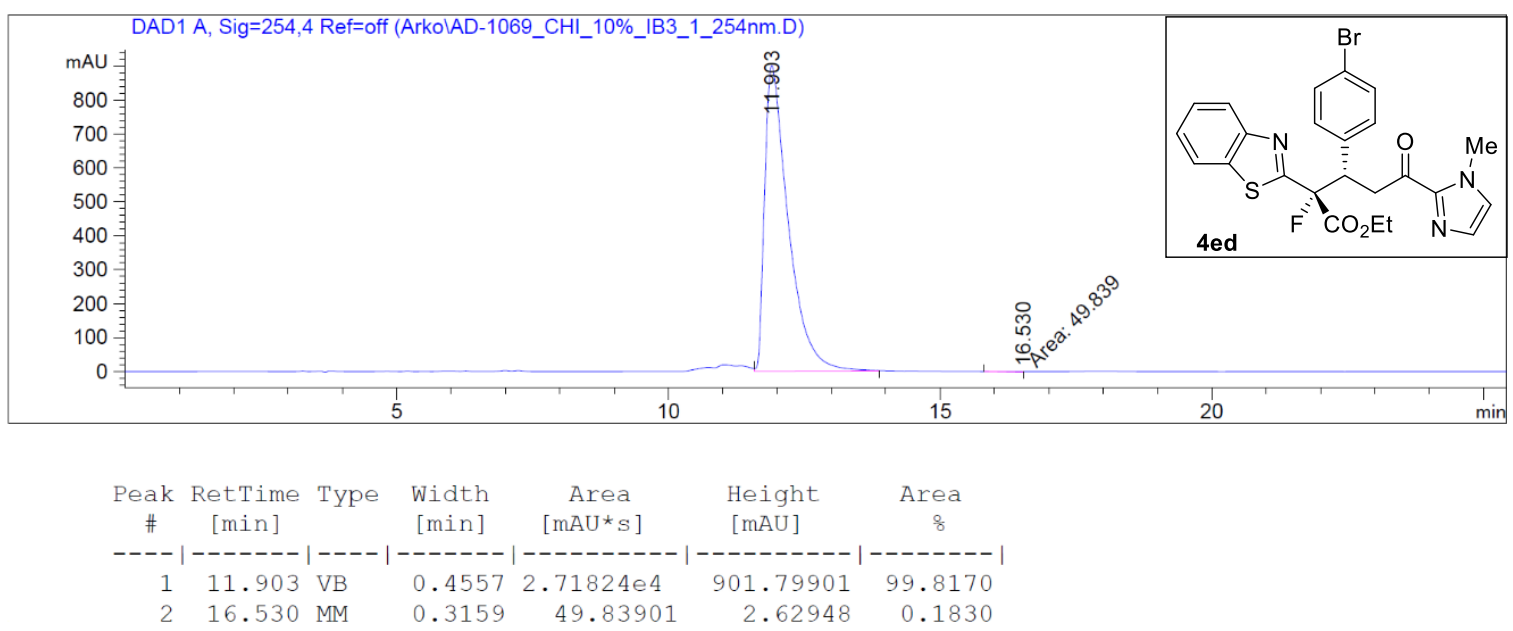

HPLC graph of chiral 4ed 


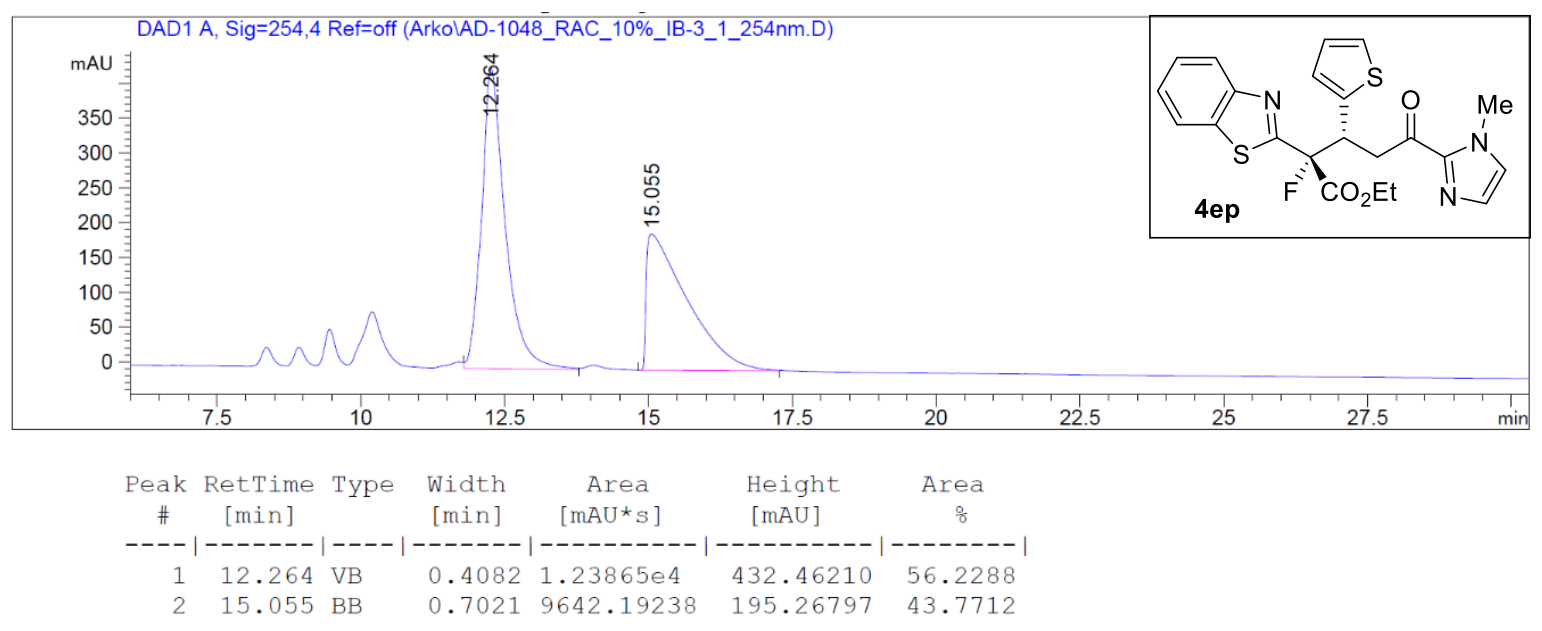

HPLC graph of racemic 4ep

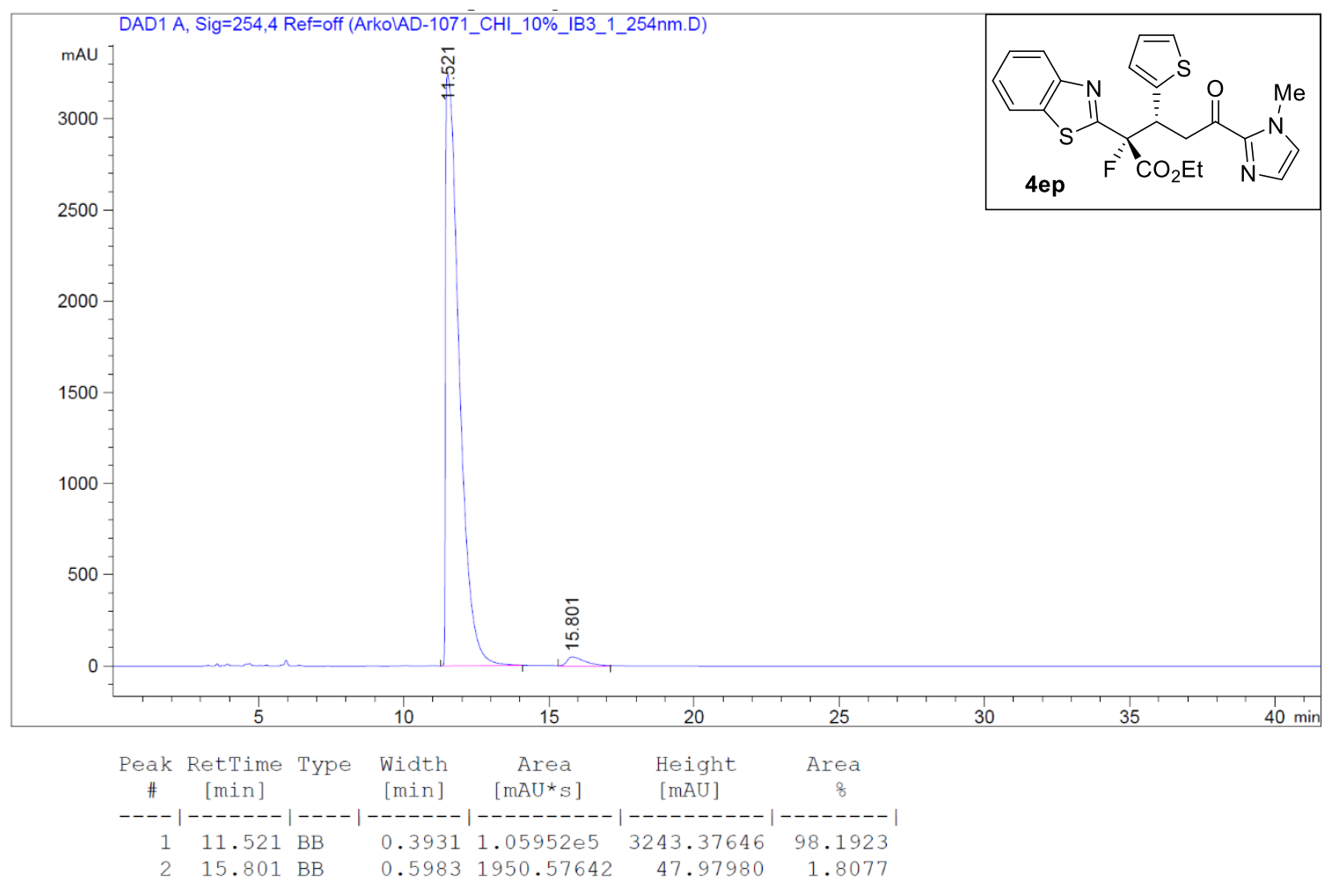

HPLC graph of chiral 4ep 


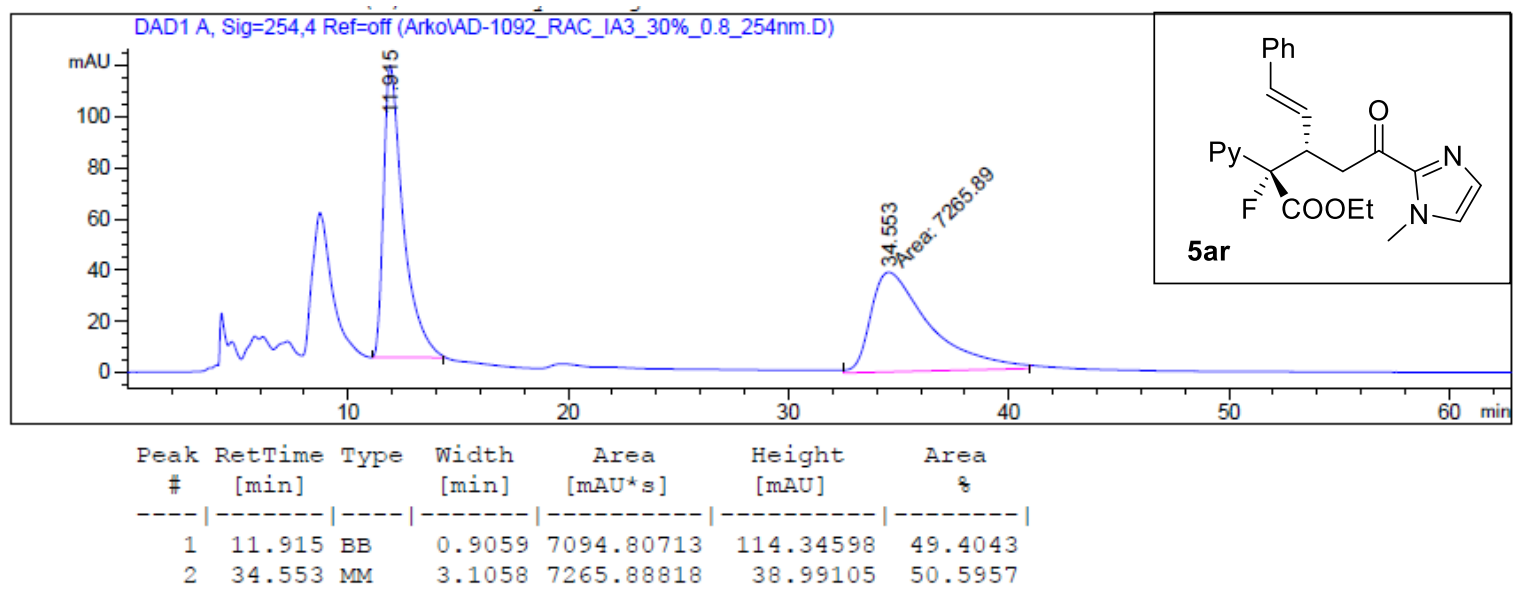

HPLC graph of racemic 5ar

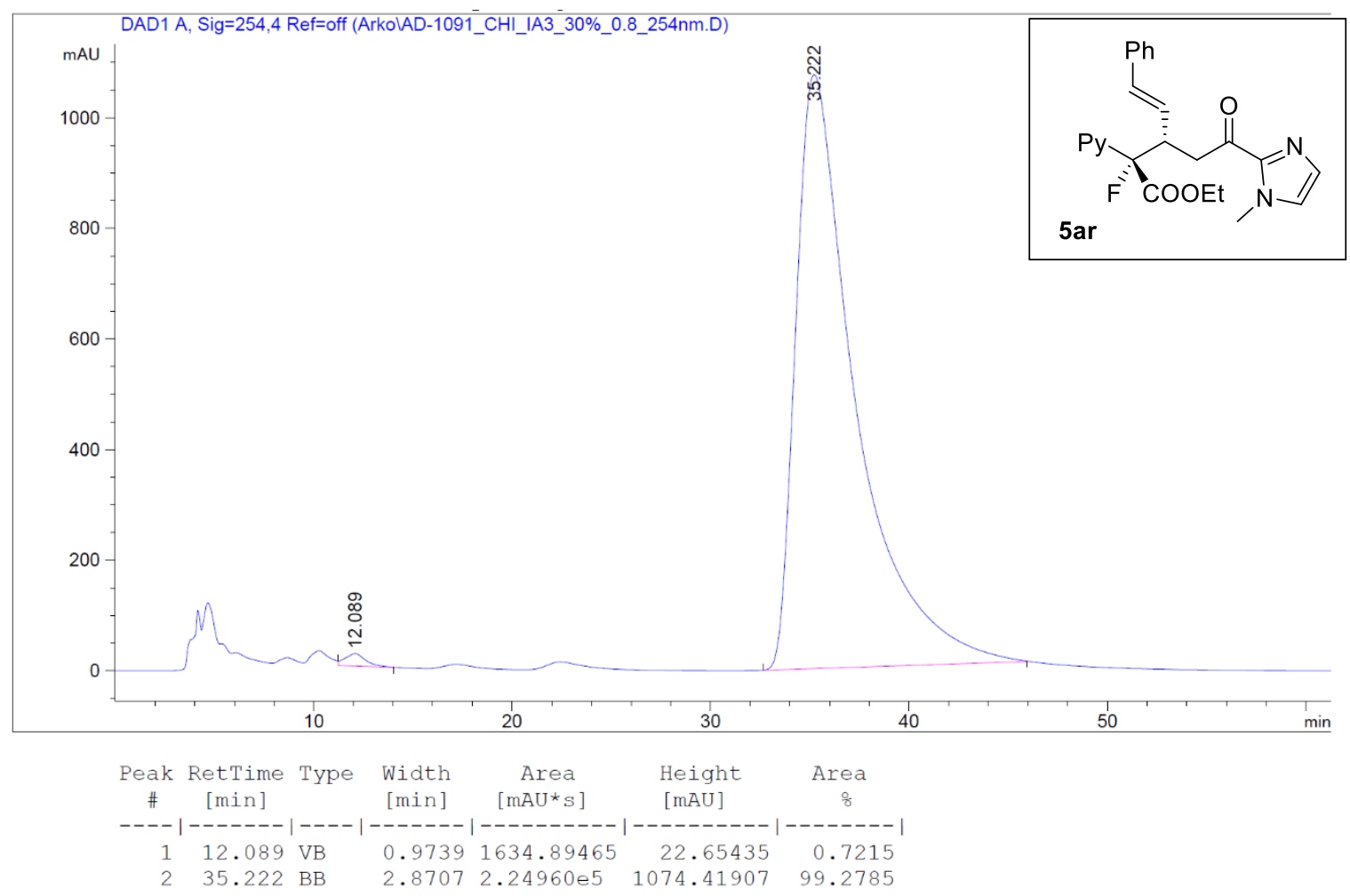

HPLC graph of chiral 5ar 


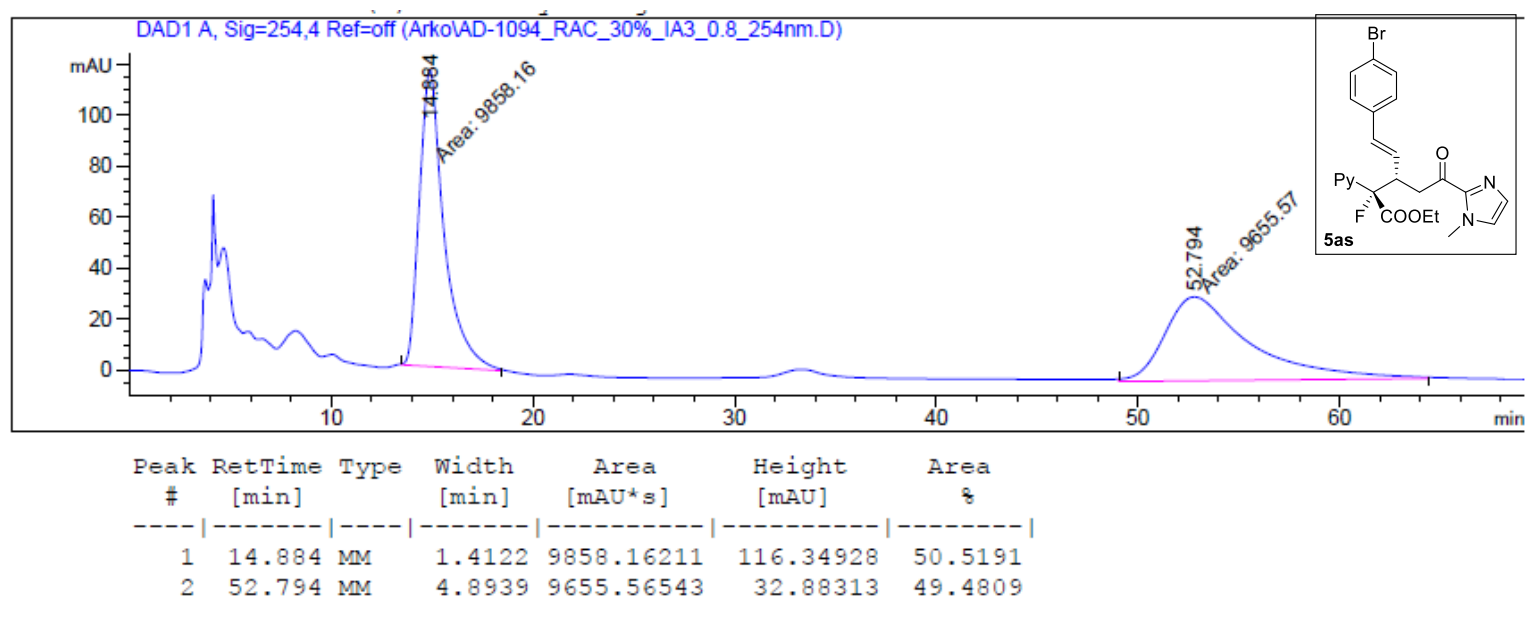

HPLC graph of racemic 5as

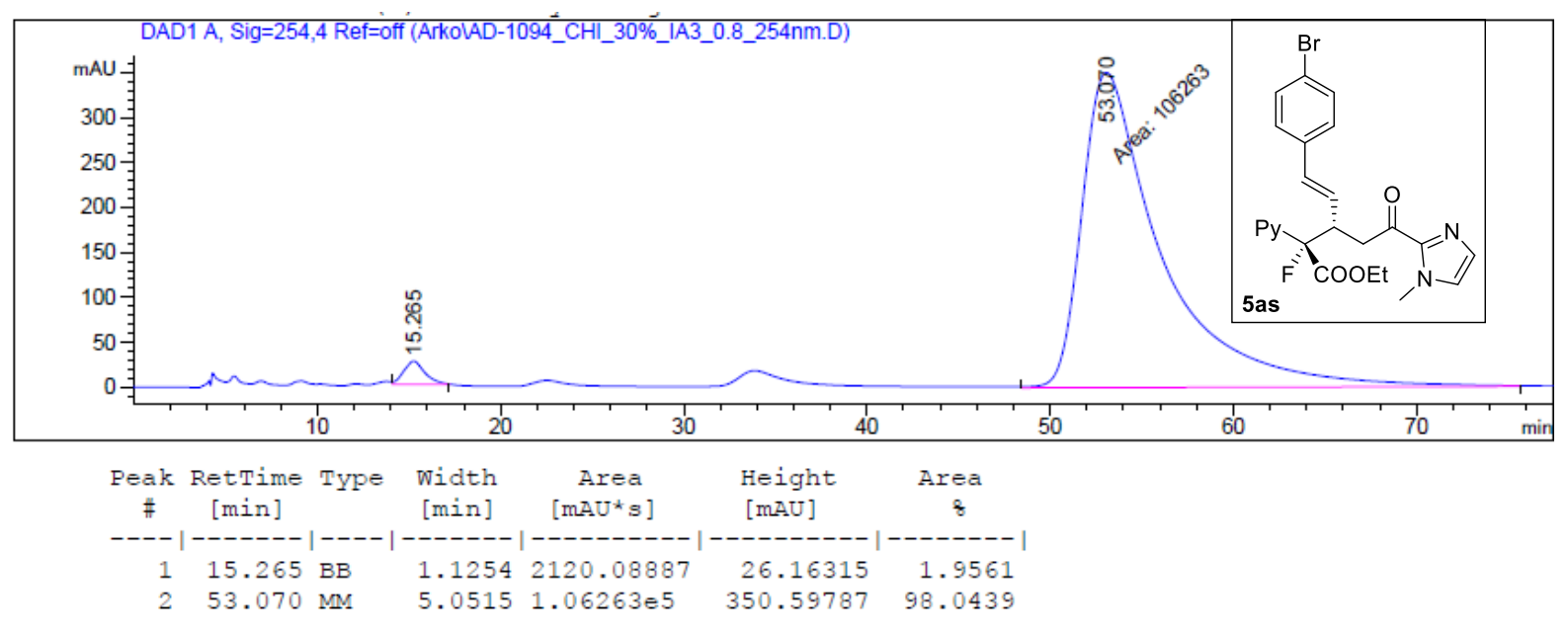

HPLC graph of chiral 5as 


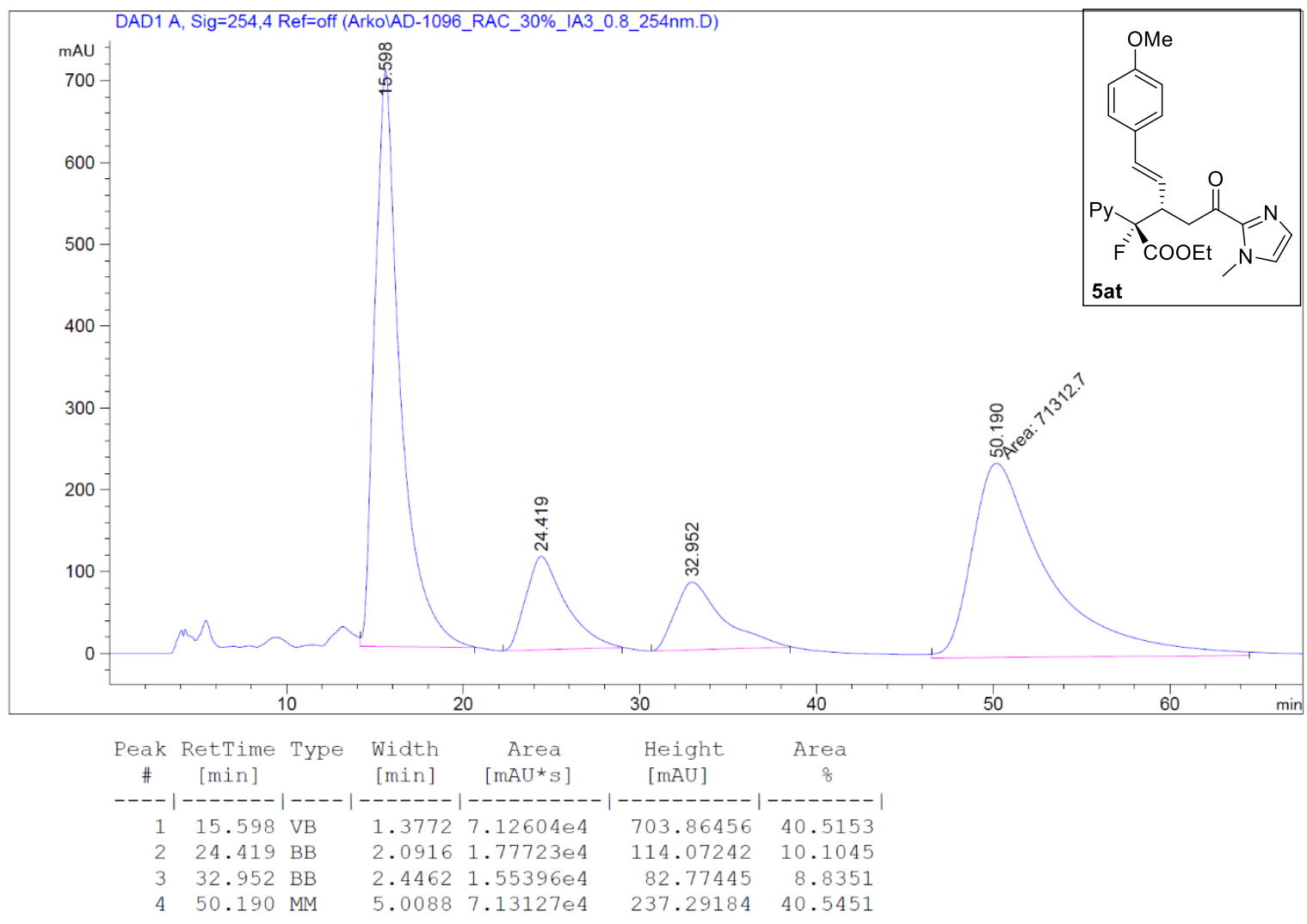

HPLC graph of racemic 5at

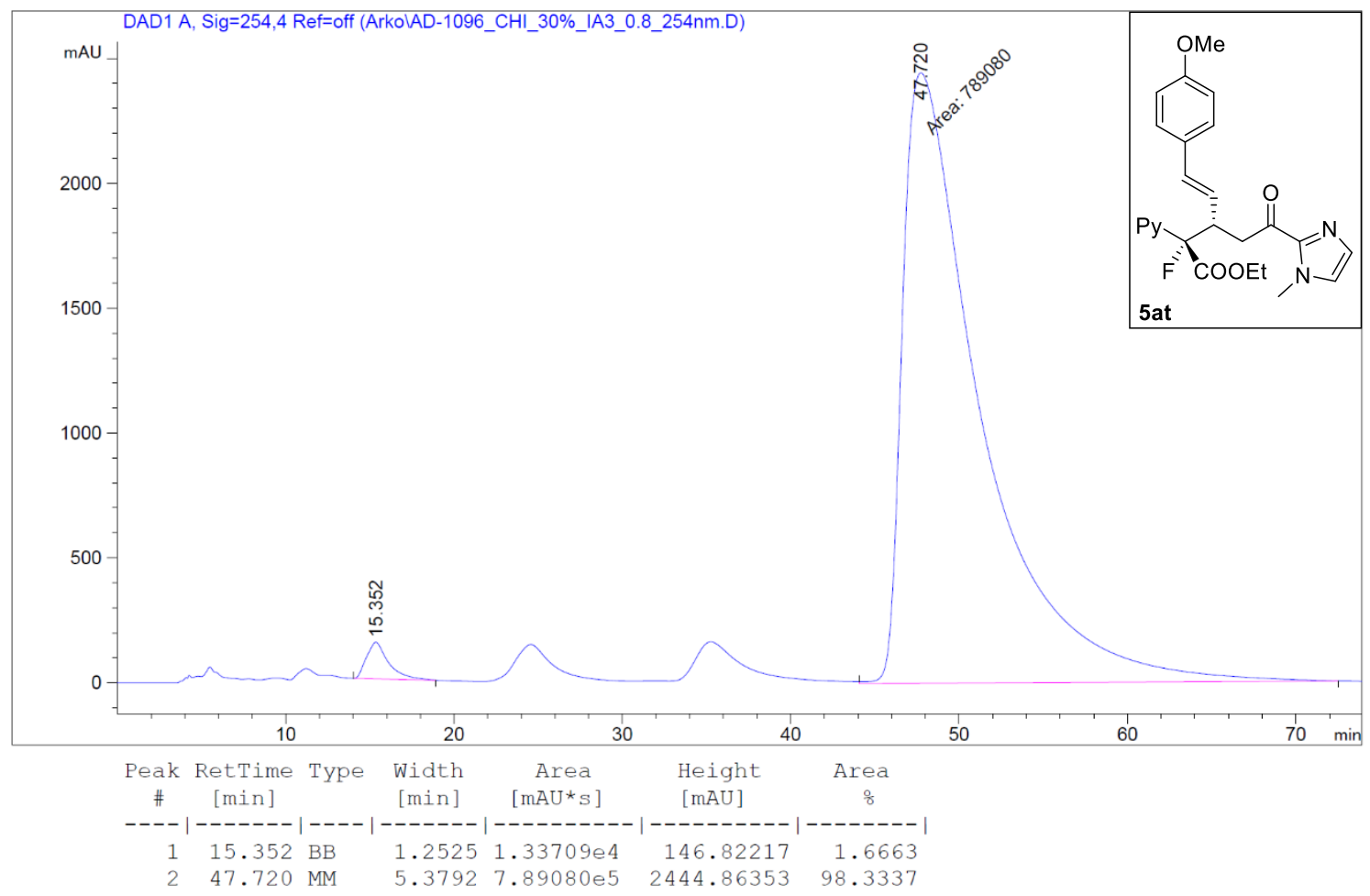

HPLC graph of chiral 5at 


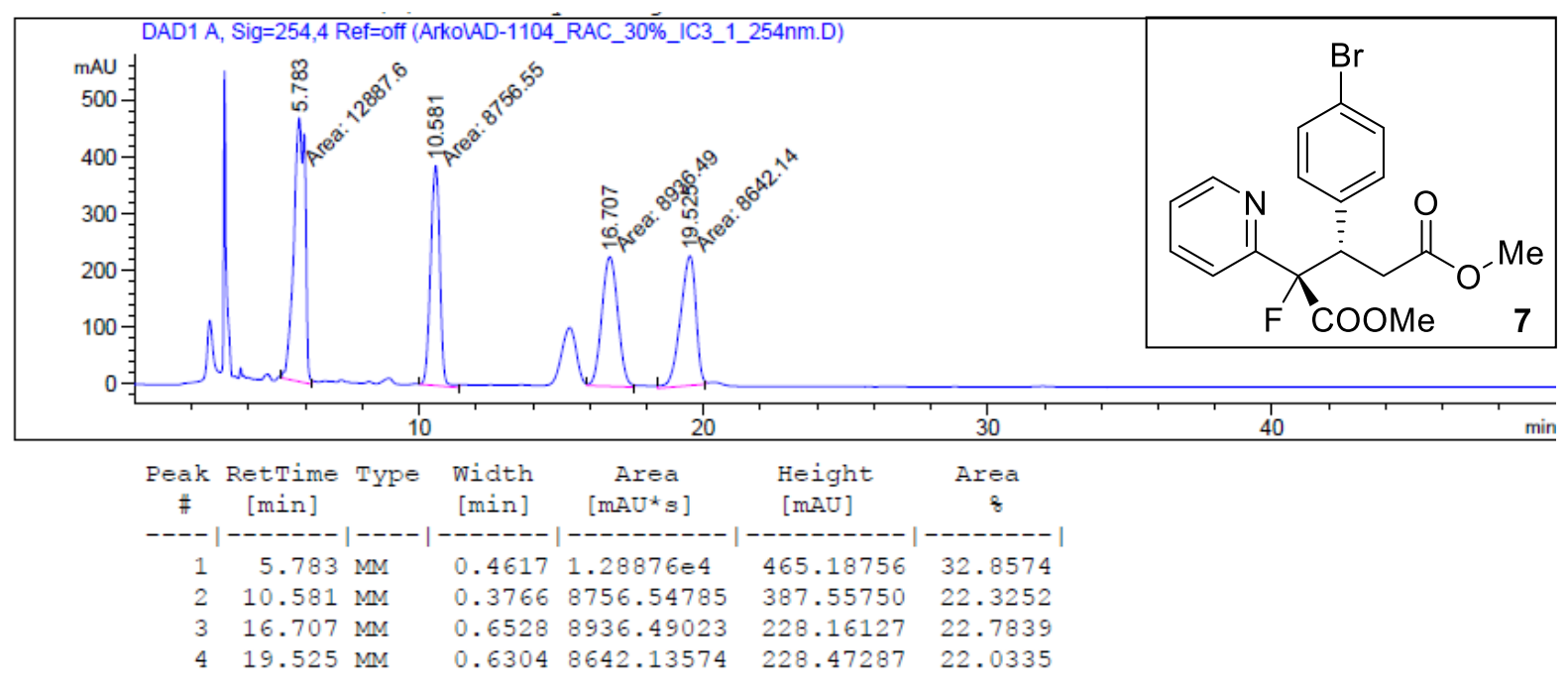

HPLC graph of racemic 7

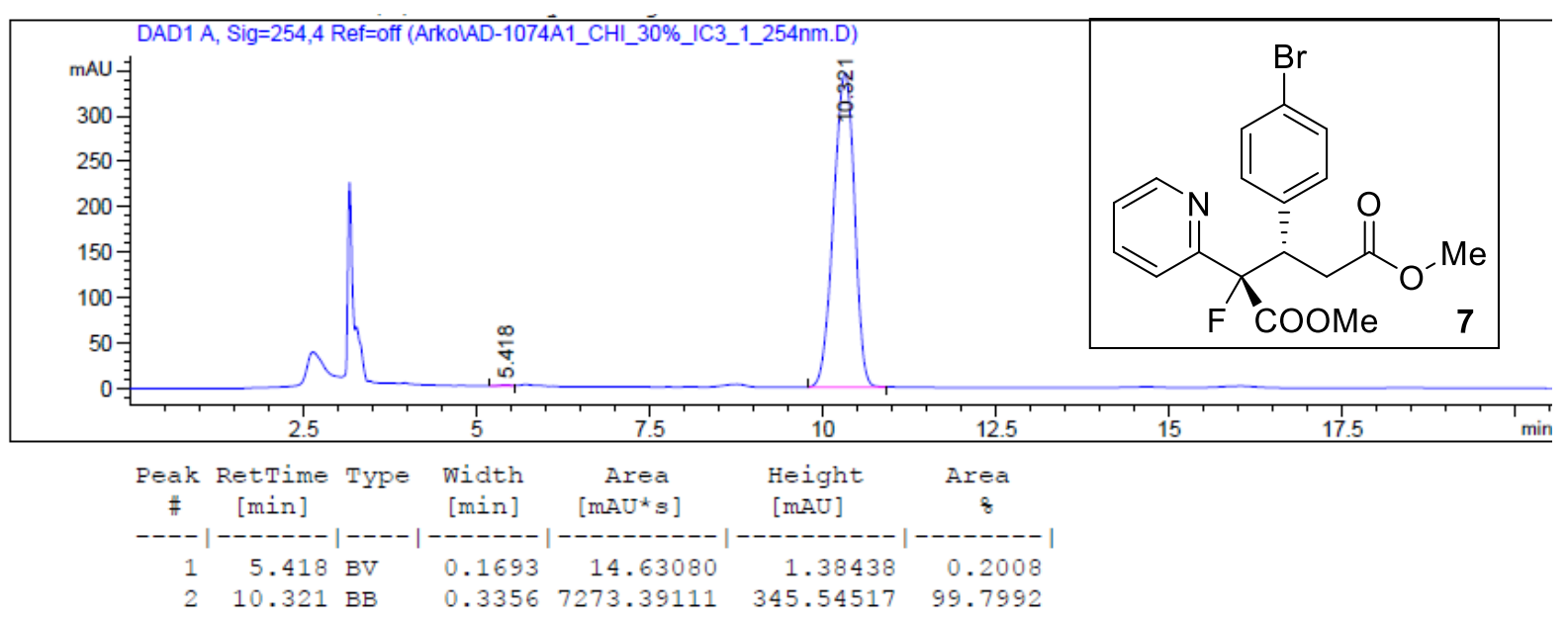

HPLC graph of chiral 7 


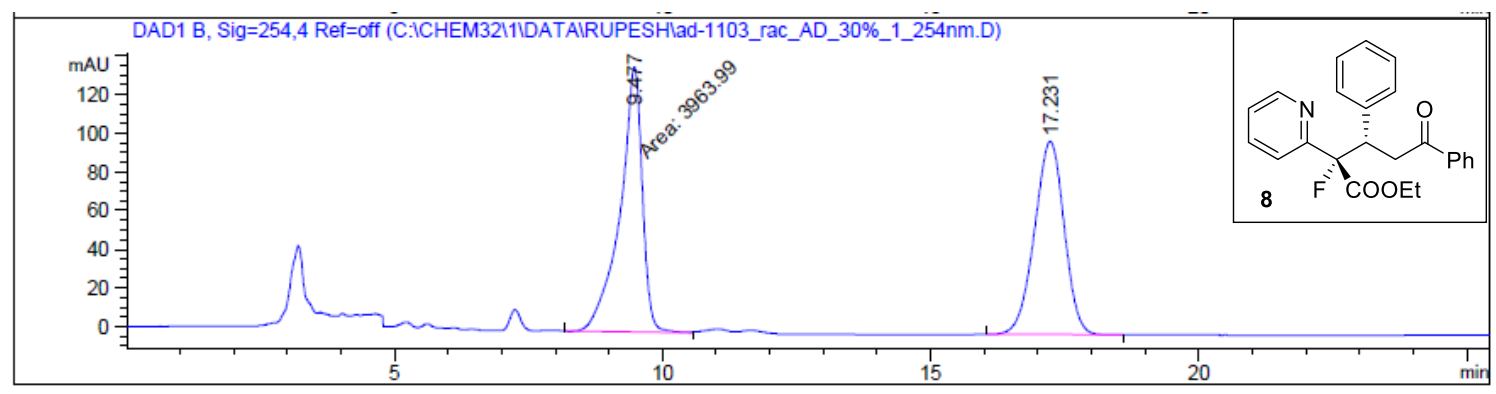

Signal 2: DAD1 B, Sig $=254,4$ Ref $=0 f f$

\begin{tabular}{cccccc}
$\begin{array}{c}\text { Peak RetTime Type } \\
\text { \# }\end{array} \begin{array}{c}\text { Width } \\
\text { [min] }\end{array}$ & $\begin{array}{c}\text { Area } \\
\text { [min] }\end{array}$ & $\begin{array}{c}\text { Height } \\
\text { [mAUs }\end{array}$ & $\begin{array}{c}\text { Area } \\
\text { [mAU] }\end{array}$ & $\%$ \\
\hline 1 & 9.477 MM & 0.4826 & 3963.98584 & 136.88329 & 50.6449 \\
2 & 17.231 BB & 0.5971 & 3863.03662 & 99.91114 & 49.3551
\end{tabular}

HPLC graph of racemic 8

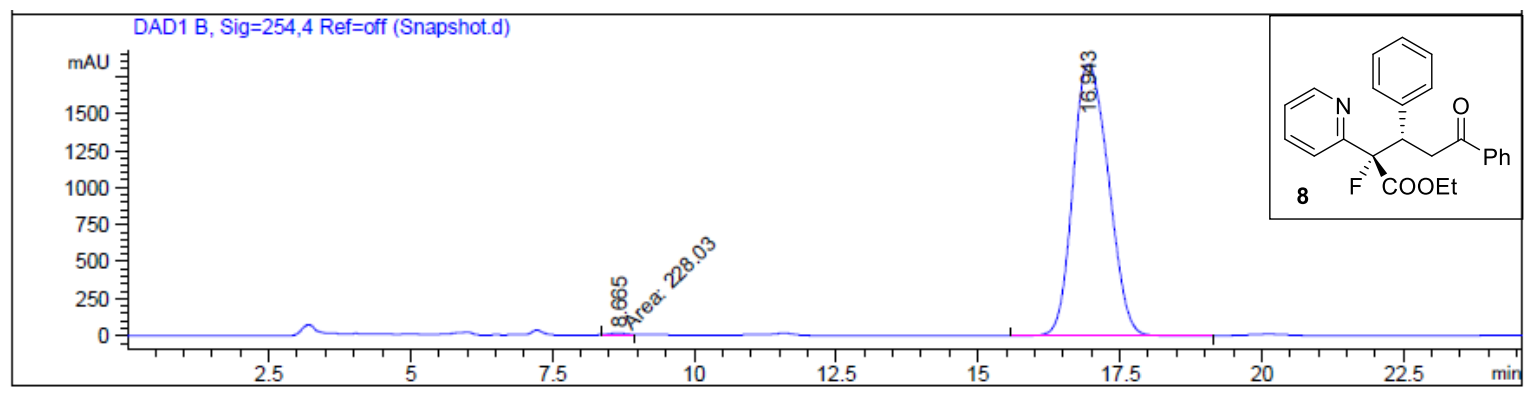

Signal 2: DAD1 B, Sig $=254,4$ Ref $=$ off

\begin{tabular}{|c|c|c|c|c|c|c|}
\hline $\begin{array}{c}\text { Peak } \\
\#\end{array}$ & $\begin{array}{l}\text { RetTime } \\
\text { [min] }\end{array}$ & Type & $\begin{array}{l}\text { Width } \\
\text { [min] }\end{array}$ & $\begin{array}{c}\text { Area } \\
{[\mathrm{mAU} * \mathrm{~s}]}\end{array}$ & $\begin{array}{l}\text { Height } \\
{[\mathrm{mAU}]}\end{array}$ & $\begin{array}{c}\text { Area } \\
\%\end{array}$ \\
\hline & & & - & 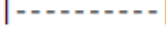 & |--...- & . \\
\hline 1 & 8.665 & 5 MM & 0.3400 & 228.03017 & 11.17830 & 0.2860 \\
\hline 2 & 16.943 & $3 \mathrm{BB}$ & 0.7026 & $7.94912 \mathrm{e} 4$ & 1830.57544 & 99.7140 \\
\hline
\end{tabular}




\section{References:}

(1) (a) Allais, C.; Liéby-Muller, F.; Constantieux, T.; Rodriguez, J. Dual Heterogeneous Catalysis for a Regioselective Three- Component Synthesis of $\mathrm{Bi}$ - and Tri(hetero)arylpyridines. Adv. Synth. Catal. 2012, 354, 2537-2544; (b) Chan, A.; Scheidt, K. A. Highly Stereoselective Formal [3 + 3] Cycloaddition of Enals and Azomethine Imines Catalyzed by N-Heterocyclic Carbenes. J. Am. Chem. Soc. 2007, 129, 5334-5335; (c) DrissiAmraoui, S.; Morin, M. S. T.; Crévisy, C.; Baslé, O.; Marcia de Figueiredo, R.; Mauduit, M.; Campagne, J.-M. Copper-Catalyzed Asymmetric Conjugate Addition of Dimethylzinc to AcylN-methylimidazole Michael Acceptors: a Powerful Synthetic Platform. Angew. Chem., Int. Ed. 2015, 54, 11830-11834; (d) Evans, D. A.; Fandrick, K. R.; Song, H.-J. Enantioselective Friedel-Crafts Alkylations of $\alpha, \beta$-Unsaturated 2-Acyl Imidazoles Catalyzed by Bis(oxazolinyl)pyridine-Scandium(III) Triflate Complexes. J. Am. Chem. Soc. 2005, 127, 8942-8943; (e) Li, S.-W.; Wan, Q.; Kang, Q. Chiral-at-Metal Rh(III) Complex-Catalyzed Michael Addition of Pyrazolones with $\alpha, \beta$-Unsaturated 2-Acyl Imidazoles. Org. Lett. 2018, 20 , 1312-1315.

(2) (a) He, Z.-T.; Jiang, X.; Hartwig, J. F. Stereodivergent Construction of Tertiary Fluorides in Vicinal Stereogenic Pairs by Allylic Substitution with Iridium and Copper Catalysts. J. Am. Chem. Soc. 2019, 141, 13066-13073; (b) Yang, S.-Q.; Wang, Y.-F.; Zhao, W.-C.; Lin, G.-Q.; He, Z.-T. Stereodivergent Synthesis of Tertiary Fluoride-Tethered Allenes via Copper and Palladium Dual Catalysis. J. Am. Chem. Soc. 2021, 143, 7285-7291; (c) Liang, J.; Han, J.; Wu, J.; Wu, P.; Hu, J.; Hu, F.; Wu, F. Nickel-Catalyzed Coupling Reaction of $\alpha-$ Bromo- $\alpha$-fluoroketones with Arylboronic Acids toward the Synthesis of $\alpha$-Fluoroketones. Org. Lett. 2019, 21, 6844-6849; (d) Duchemin, N.; Cattoen, M.; Gayraud, O.; Anselmi, S.; Siddiq, B.; Buccafusca, R.; Daumas, M.; Ferey, V.; Smietana, M.; Arseniyadis, S. Direct Access to Highly Enantioenriched $\alpha$-Branched Acrylonitriles through a One-Pot Sequential Asymmetric Michael Addition/Retro-Dieckmann/Retro-Michael Fragmentation Cascade. Org. Lett. 2020, 22, 5995-6000. 